\title{
Lipid organisation and dynamics in the myelin membrane sheets
}

\section{PhD thesis}

in partial fulfilment of the requirements

for the degree Doctor rerum naturalium (Dr. rer. nat.)

in the Molecular Biology Program

at the Georg-August University Göttingen,

Faculty of Biology

submitted by

Olena Steshenko

born in

Kiev, Ukraine 

And I asked them, "So what are your concerns?" "Same as the whole science concerns are, answered a hawk-nosed man, the human happiness'

Monday Begins on Saturday, Strugatski Brothers, 1964 

Thesis Committee:

Prof. Mikael Simons

Prof. Silvio Rizzoli

Prof. Jörg Enderlein 



\section{Affidavit}

I hereby declare that this $\mathrm{PhD}$ thesis "Lipid organisation and dynamics in the myelin membrane sheets" has been written independently with no other aids or sources than quoted. This thesis (whole or in parts) has not been submitted elsewhere for any academic purposes.

\section{Olena Steshenko}

Submission date: July 23, 2014

Signature

Göttingen, Germany 



\section{Contents}

$\begin{array}{ll}\text { Acknowledgements } & \text { ix }\end{array}$

Abstract $\quad$ xi

List of figures $\quad$ xii

List of tables $\quad$ xiv

1 Introduction $\quad 1$

1.1 Myelin origin in CNS: oligodendrocytes . . . . . . . . . . . . 3

1.1.1 Developmental characteristics of oligodendrocytes . . . . . . . 3

1.1.2 Myelination onset . . . . . . . . . . . . . . 6

1.2 Myelin structure . . . . . . . . . . . . . . . . . 7

1.3 Myelin lipid composition . . . . . . . . . . . . . . . 8

1.3.1 Glycerolipids and glycerophospholipids . . . . . . . . . . . . . 9

1.3.2 Phosphatidylinositol, PtdIns . . . . . . . . . . . . . . . 13

1.3.3 Sphingolipids . . . . . . . . . . . . . . . . . 17

1.3 .4 Cholesterol . . . . . . . . . . . . . . . . . . 19

1.3.5 Fatty acid composition of myelin lipids . . . . . . . . . . 20

1.4 Myelin protein composition . . . . . . . . . . . . . . 21

1.4.1 Myelin basic protein, MBP . . . . . . . . . . . . . . 21

1.4.2 2/,3/-cyclic-nucleotide phosphodiesterase, CNP . . . . . . . 23

1.4.3 Proteolipid protein, PLP . . . . . . . . . . . . 24

1.4.4 Myelin oligodendrocyte glycoprotein, MOG . . . . . . . . . . 25

1.4.5 Myelin-associated glycoprotein, MAG . . . . . . . . . . 25

1.5 Lipid behaviour and their dynamical properties within the membrane 26

1.5.1 Historical overview of the cell membrane architecture investigations . . . . . . . . . . . . . 26

1.5.2 Types of lipid dynamics . . . . . . . . . . . . . . 27

1.5.3 Myelin as a unique membrane in a context of the lipid diffusion 32

1.6 Cell polarity . . . . . . . . . . . . . . . 33

1.6.1 Overview of the mechanisms of the cellular polarisation . . . . 34

1.6.2 Polarity within the myelin sheath . . . . . . . . . . . . 37

1.7 Aim of the work . . . . . . . . . . . . . . . . . . . 40

2 Materials \& Methods $\quad 41$

Materials $\quad 43$

2.1 Chemicals and Consumables . . . . . . . . . . . . . . . 43

2.2 Antibodies . . . . . . . . . . . . . . . . . . . . . 43

2.3 Specific Software . . . . . . . . . . . . . . . . . . 44 
Methods 45

2.4 Cell culture . . . . . . . . . . . . . . . . . . . . 45

2.4.1 Primary oligodendrocyte culture . . . . . . . . . . . . . . 45

2.4.2 PtK2 fibroblast cell line . . . . . . . . . . . . 46

2.4.3 Nucleic acids delivery into cultured animal cells . . . . . . . . . 46

2.4 .4 PtK2-MBP assay . . . . . . . . . . . . . . . 47

2.5 Inhibitors administration . . . . . . . . . . . . . . . . 47

2.5.1 PTEN inhibition . . . . . . . . . . . . . . . . . . 47

2.5.2 Ionomycin treatment . . . . . . . . . . . . . . . . 47

2.6 Immunocytochemistry . . . . . . . . . . . . . . . . . . . . . . . . . . 48

2.6.1 Labelling with the small protein probes . . . . . . . . . . . . . 49

2.6.2 Delivery of $\mathrm{PIP}_{3} \quad \ldots \ldots \ldots \ldots$. . . . . . . . . . . . . . . . . . . . . . . . . 49

2.7 Biochemistry . . . . . . . . . . . . . . . . . . . . . . . 49

2.7.1 SDS-PAGE - Sodium Dodecyl Sulphate Polyacrylamide Gel

Electrophoresis . . . . . . . . . . . . . . . . . 49

2.7.2 Western Blot . . . . . . . . . . . . . 50

2.7.3 Quick Coomassie ................. 51

2.7.4 Preparation of large unilamellar vesicles (LUVs) and their pulldown with MBP ................. 51

2.7.5 Soluble/insoluble actin fractionation . . . . . . . . . . 52

2.7.6 BSA-coupling of artificial lipids . . . . . . . . . . . . . . . 52

2.7.7 Incorporation of the artificial lipids . . . . . . . . . . . 53

2.8 Microscopy . . . . . . . . . . . . . . . . . . . 54

2.8.1 STED-FCS Microscopy . . . . . . . . . . . . . . . 54

2.8.2 Confocal Microscopy . . . . . . . . . . . . . . . . . 57

2.9 Image Processing and Statistical Analysis . . . . . . . . . . . . . . 57

3 Results $\quad 59$

Part I: Lipid dynamics and organisation in the myelin membrane sheets 61

3.1 Cultured oligodendrocytes as a model system . . . . . . . . . . . . 61

3.2 Unique features of myelin membrane sheath that influence lipid behaviour . . . . . . . . . . . . . . . . 62

3.3 Lipid diffusion in oligodendrocyte sheets versus PtK2 cell membrane . 64

3.4 Mimicking the oligodendrocyte sheets in PtK2 cells . . . . . . . . . . 68

3.5 Effects of fatty acid chain length . . . . . . . . . . . . . . 73

Part II: Polarity of the phosphatidylinositols in the oligodendrocytes $\mathbf{7 5}$

3.6 Distribution of phosphatidylinositols in oligodendrocytes . . . . . . . 75

3.6.1 Defining the differential localisation of $\mathrm{PIP}_{2}$ and $\mathrm{PIP}_{3}$ in the mature oligodendrocytes . . . . . . . . . . . . . 75

3.6.2 Defining the onset of the separate localisation of phosphatidylinositols . . . . . . . . . . . . . . 81

3.6.3 Role of MBP in the localisation of phosphatidylinositols . . . 81

3.6.4 MBP interactions with differently charged phosphatidylinositols 81

$3.7 \mathrm{PIP}_{3}$ influences non-compact region of the oligodendrocytes . . . . . 86

3.7.1 Interference with the endogenous $\mathrm{PIP}_{3}$ levels in the oligodendrocytes .................... 86 
3.7.2 Actin as a downstream effector of the $\mathrm{PIP}_{3}$ signalling . . . . . 92

$\begin{array}{lll}4 & \text { Discussion } & 97\end{array}$

Part I: Characterisation of lipid diffusion in oligodendrocytes $\quad 99$

4.1 FCS-STED to study lipid behaviour . . . . . . . . . . . . . . . . . . . . . 99

4.1.1 Impact of MBP on lipid diffusion . . . . . . . . . . . . . . 100

4.1.2 Length of the fatty-acid chain in myelin . . . . . . . . . . . 101

Part II: Role of phosphatidylinositols in the cellular polarity 103

4.2 Role of phosphatidylinositols in the cellular polarity . . . . . . . . . 103

4.2.1 Mature oligodendrocytes show distinct localisation of the phosphatidylinositols . . . . . . . . . . . . . . 104

4.2.2 Early-stage oligodendrocytes, and those from shiverer mice, lack differential localisation of the phosphatidylinositols . . . . 104

4.2.3 MBP as a phosphatidylinositols'-separating protein . . . . . . 105

4.2.4 Compact/non-compact domain ratio can be altered upon augmentation of $\mathrm{PIP}_{3}$ in the oligodendrocytes . . . . . . . 106

4.2.5 Increased levels of $\mathrm{PIP}_{3}$ and actin might regulate the size of the non-compact domains . . . . . . . . . . . . . 108

5 Summary and Conclusions $\quad 111$

$\begin{array}{ll}\text { References } & 115\end{array}$

Curriculum Vitae 



\section{Acknowledgements}

My sincere gratefulness primary is addressed to my scientific advisor, Prof. Dr. Mikael Simons, for the opportunity to accomplish my $\mathrm{PhD}$ thesis in his group, for his supervision, open mind, support of my ideas, guidance through the pitfalls of the scientific everyday life and for providing with an outstanding working environment in his group in Max-Planck Institute for experimental medicine. I would like to thank the members of my thesis committee, Prof. Dr. Silvio O. Rizzoli and Prof. Dr. Jörg Enderlein for their time, fruitful discussions and scientific guidance.

I would like to cordially thank to our collaborators, first of all, Dr. Christian Eggeling, for always finding time for discussions and for the optimism and enormous motivation he provided me with. Next, my thank goes to the two PhD students, Debora M. Andrade and Veronika Müller, whose brilliant experience in the field of FCS and STED provided me with a necessary support to accomplish the project.

Furthermore, I give my cordially thank to the Coordination Office of $\mathrm{MSc} / \mathrm{PhD}$ Molecular Biology Program: Steffen Burkhardt and his assistants Ivana Bacakova and Kerstin Grüniger for the great opportunity to come to Göttingen in the first place and to be part of the IMPRS Graduate School. The competence and tremendous skills of the Coordination Office made a transition to the cultural and scientific life in Germany very smooth and easy; they contributed much to make me feel here, in Göttingen at home. My special gratitude and praise goes to Steffen Burkhardt, for his help, suggestions, and almost parental support during my master studies and PhD-time.

It was a pleasure to work in a fantastic collective of my lab comrades in the AG Simons, especially with such a brilliant co-workers as Shweta Aggarwal, Natalia Manrique, Mostafa Bakhti, Sebastian Schmidt, Caroline Velte, Marie-Theres Weil. These guys were always there with a piece of chocolate and with a cup of coffee when things were going wrong to cheer-up, and, most importantly, they were also there to share the happiness and the positive results. Special thanks to Gieselheid Schulz for providing cell culture in the times of "cell-culture-crisis". Exclusively I would like to note Larisa Yurlova, who was my lab-rotation supervisor in the years of Master studies, and thanks to whom I fell in deep love with the oligodendrocytes and myelin.

I am grateful to the Open Source software developers, i.e. ImageJ and $\mathrm{AT}_{\mathrm{E} X}$, for making writing of my thesis manuscript such a fun and easy thing.

I would like to express my gratefulness to the TSC Schwarz-Gold Göttingen Latin A-Team, especially to Markus Welzel, Peter Rupp, Merrit Dahme, Franzi Degen, Juliane Vey, Tobias Müller (Mü), Leon Falke, Nastja Shishkina and Martina Lotsch, for the fun and unforgettable extracurricular activities, stunning trainings and crazy evenings/nights/weekends spent together and to Maurice Flügge and Ralph Krimmel for being my superb dancing partners and for all the trophies we won together.

I want to express my appreciation to my dear friends - Oleksandra Karpiuk, Liudmila Filonava and Hanna Peradziryi - for the awesome time together, wise scientific advises, fruitful discussions and just random girly coffee-talks, for the understanding 
and support.

My deepest gratitude among all, goes to a very special person in my life - Aliaksander Dzementsei, who managed to stand next to crazy me the whole $\mathrm{PhD}$-time and I hope would do this further on. I thank him for his moral strength, wise silence and appropriate decisions, for sharing my triumphs as well as my darkest hours and never leaving me alone.

I am grateful to my dear parents - Volodymir and Natalia Steshenko and to my beloved sister Yulia that always supported my decisions from Kiev.

Last, but not least, I would like to thank my former teachers in Kiev National Taras Schevchenko University, who planted the seed of love to life science in my heart and helped it to grow and develop. 


\section{Abstract}

Myelin is a unique lipid-enriched membrane, produced by oligodendrocytes in the central nervous system, that is enwrapped around axons in the multilayered fashion to ensure a swift saltatory conduction of the neuronal impulses.

Myelin has a unique composition. It is enriched in the cholesterol, sphingomyelin and myelin lipids possess high number of long-chain fatty acids in their structure. Previous results have shown that myelin basic protein (MBP) acts as a diffusion barrier within myelin sheath, preventing the majority of the proteins to enter the sheath region. Therefore, myelin sheaths are deprived of cytoplasm and proteins, and do not contain cortical actin cytoskeleton. This specific composition raises the question of how lipid diffusion might be affected in the myelin sheath.

We used primary oligodendrocyte culture as a well-established model to study lipid diffusion in myelin sheath. To assess lipid diffusion modes, we used STED-FCS (stimulated emission depletion microscopy combined with fluorescence correlation spectroscopy). We showed that artificial analogs of phosphatidylethanolamine, sphingomyelin and galactosylcerebroside (PE, SM and GalC, respectively) introduced into the cellular membrane, have a faster diffusion within oligodendrocytes, when compared to the diffusion within control membrane of the fibroblasts cell line (PtK2 cells). Moreover, we could show a complete absence of trapping for SM and a decrease in the trapping for $\mathrm{GalC}$ in the membrane of oligodendrocytes. Additionally, using a model of the reconstituted oligodendrocyte sheets in PtK2 cells, we could demonstrate that MBP presence is sufficient to reorganize cellular membrane and change the mode of the lipids behaviour, from trapping to the free diffusion.

In the second project we addressed the question of the polarised distribution of phosphatidylinositols in oligodendrocyte membrane. We could demonstrate a distribution of $\mathrm{PIP}_{2}$ and $\mathrm{PIP}_{3}$ into two distinct cellular regions: sheets for $\mathrm{PIP}_{2}$ and outer rim and processes for $\mathrm{PIP}_{3}$. We showed that MBP interacts with $\mathrm{PIP}_{2}$ in the sheets and masks it from antibody staining, but not with $\mathrm{PIP}_{3}$ in the processes. Furthermore, we found that oligodendrocytes are highly susceptible to the changes in the $\mathrm{PIP}_{3}$ levels: upon its augmentation the size of non-compact domains within oligodendrocytes increased. We found that an increase of actin accompanied an with enlargement of the non-compact regions. We speculate that this augmentation of the filamentous actin is a reason that would prevent MBP from zipping the non-compact regions. We suggest actin to be a downstream effector of the increased $\mathrm{PIP}_{3}$ levels. 



\section{List of Figures}

1.1 Developmental stages of oligodendrocyte in culture. . . . . . . . . 5

1.2 Schematic overview of the myelin architecture in vivo. . . . . . . . . . 7

1.3 Lipid distribution within the membrane leaflets. . . . . . . . . . . . . 13

1.4 Proteins distribution in myelin sheath. . . . . . . . . . . . . . 21

1.5 Schematic overview of the membrane models. . . . . . . . . . . . . 28

1.6 Models of the membrane dynamics. . . . . . . . . . . . . . . . . 29

1.7 Trapping and hopping models. . . . . . . . . . . . . . . . . . . 31

1.8 Major complexes involved in cell polarity regulation. . . . . . . . . . 38

1.9 Polarised domains of the myelin sheath. . . . . . . . . . . . . . 39

2.1 Schematic representation of the MBP-GFP-TM construct, used for the PtK2 transfection to reproduce MBP membrane zipping in the cell line. . . . . . . . . . . . . . . . . . . . . . . . . 4 47

3.1 Domains of oligodendrocytes cultivated in vivo. . . . . . . . . . . . 62

3.2 Diffusion coefficient of the PE, SM and GalC in oligodendrocytes is higher than in PtK2 cells. . . . . . . . . . . . . . . . . 64

3.3 Diffusion ratio of PE, SM and GalC in oligos is higher than in PtK2 cells. . . . . . . . . . . . . . . . . 66

3.4 FCS diffusion plots for SM and PE in oligodendrocytes demonstrate free diffusion mode, and decrease of trapping for GalC . . . . . . . . . 67

3.5 Oligodendrocyte sheets and sheets-like regions within PtK2-MBP+ cells are free of actin and glycoproteins. . . . . . . . . . . . . 69

3.6 Diffusion coefficient of the PE, SM and GalC in PtK2-MBP + regions is higher than in oligodendrocyte sheets and control PtK2 cells. . . . 70

3.7 Diffusion ratio of the PE, SM and GalC in PtK2-MBP + regions is higher than in oligodendrocyte sheets and control PtK2 cells. . . . . . 71

3.8 FCS diffusion plots for SM, PE and GalC in $\mathrm{PtK} 2-\mathrm{MBP}+$ regions demonstrate absence of the anomalous diffusion. . . . . . . . . . 72

3.9 Diffusion characteristics for 23C-GalC in PtK2 control and PtK2$\mathrm{MBP}+$ cells. . . . . . . . . . . . . . . . . . . . 74

3.10 Fixative agents extract $\mathrm{PIP}_{2}$ and $\mathrm{PIP}_{3}$, decreasing efficiency of antibody labelling. . . . . . . . . . . . . . . . . . . 77

$3.11 \mathrm{PIP}_{2}$ and $\mathrm{PIP}_{3}$ are found in the processed and in the rim of mature oligodendrocytes. . . . . . . . . . . . . . . . 78

3.12 $\mathrm{PIP}_{2}$, but not $\mathrm{PIP}_{3}$ is masked by MBP in the sheets of oligodendrocytes. 79

$3.13 \mathrm{PIP}_{3}$ localises strictly to the processes and the rim in oligodendrocytes. 80

$3.14 \mathrm{PIP}_{2}$ and $\mathrm{PIP}_{3}$ localise to the sheets at the early stages of oligodendrocytes. . . . . . . . . . . . . . . . . . . . 82

3.15 $\mathrm{PIP}_{3}$ localisation changes from uniformly distributed to processes and rim-enriched upon oligodendrocytes maturation. . . . . . . . . . . 
3.16 $\mathrm{PIP}_{2}$ and $\mathrm{PIP}_{3}$ are uniformly distributed in the membrane of oligodendrocytes from shiverer mice. . . . . . . . . . . . . . 84

3.17 Pull-down of MBP by liposomes with different negatively charged lipids composition did not reveal preference of MBP binding to any of the phosphatidylinositols. . . . . . . . . . . .

3.18 Treatment with $200 \mathrm{pM}$ PTEN inhibitor leads to the increased amount of endogenous $\mathrm{PIP}_{3}$ in oligodendrocytes on day $3 \ldots \ldots \ldots . . .87$

3.19 Treatment with $200 \mathrm{pM}$ PTEN inhibitor leads to the increased amount of oligodendrocytes with CNP-positive, but yet MBP-negative sheets on day $2 \ldots \ldots \ldots \ldots$

3.20 Treatment with $100 \mathrm{nM}$ PTEN inhibitor leads to an increase of rim size, as well as an increase of the non-compact regions. . . . . . . 89

3.21 Treatment with $200 \mathrm{pM}$ PTEN inhibitor leads to the increased amount of phosphoAkt in oligodendrocytes on day $3 \ldots \ldots$. . . . . . . 90

3.22 Treatment with $100 \mathrm{nM}$ PTEN inhibitor leads to the increase of noncompact region and decrease of the compact domain in oligodendrocytes on day $3 . \ldots \ldots \ldots \ldots \ldots \ldots \ldots \ldots$

3.23 Delivery of the exogenous $\mathrm{PIP}_{3}$ did not influence neither morphology, nor actin amount in the oligodendrocytes on day $3 \ldots$. . . . . . . 93

3.24 Treatment with different concentrations of PTEN inhibitor did not affect filamentous actin amount in oligodendrocytes on day 3. . . . . 94

3.25 Treatment with $100 \mathrm{nM}$ PTEN inhibitor resulted in increase of filamentous actin amount in oligodendrocytes on day $5 \ldots$. . . . . . 95

4.1 Differences between oligodendrocytes and PtK2 cell membrane. . . 102

4.2 Model of phosphatidylinositols unmixing by MBP. . . . . . . . . 107

4.3 Model of the PTEN inhibition effect on the compact/non-compact domain development. . . . . . . . . . . . . . . . . . . 109 


\section{List of Tables}

1.1 Markers of oligodendrocytes development. . . . . . . . . . . . . . . . 4

1.2 Comparison of the human and rat brain composition. . . . . . . . . 9

1.3 Structure of glycerophospholipids. . . . . . . . . . . . . . . . . . 10

1.4 Structure of sphigolipids. . . . . . . . . . . . . . . . . . . 18

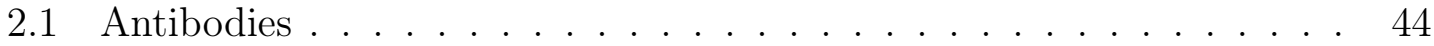

2.2 List of software . . . . . . . . . . . . . . . . . . . . . 44

2.3 SuperSato composition . . . . . . . . . . . . . . . . 45

2.4 Phosphate Buffered Saline (PBS) . . . . . . . . . . . . . . . . . 46

2.5 PtK2-Sato . . . . . . . . . . . . . . . . . . 46

2.6 Krebs-Ringer solution . . . . . . . . . . . . . . . . . . . . . . 48

2.7 1X Tris-buffered saline, TBS . . . . . . . . . . . . . . . . . . . 49

2.8 Stacking gel for SDS-PAGE . . . . . . . . . . . . . . . 50

2.9 Resolving gel for SDS-PAGE . . . . . . . . . . . . . . . . . . 50

2.10 Non-reducing sample buffer . . . . . . . . . . . . . . . . 50

2.11 Transfer buffer . . . . . . . . . . . . . . . . . . 51

2.12 Solutions for Quick Coomassie . . . . . . . . . . . . . 51

2.13 Triton-extraction buffer . . . . . . . . . . . . . . . 52

2.14 RIPA-extraction buffer . . . . . . . . . . . . . . . . . . 53

2.15 Artificial lipids for STED-FCS . . . . . . . . . . . . . . 53 

Chapter 1

\section{INTRODUCTION}

If you can't explain it simply, you don't understand it well enough.

Albert Einstein 

Myelin is a lipid-rich membrane structure, spirally wrapped around axons, insulating them from the extracellular environment. The term myelin has been introduced by Virchow in the early 50-ties of the $19^{\text {th }}$ century (Virchow, 1854). Myelin is produced by oligodendrocytes in the central nervous system (CNS) and by the Schwann cells in the peripheral nervous system (PNS). These cells send their cellular extensions (processes) to the neuronal axons and engulf them with special membrane structures - sheaths, formed upon the contact of every process with an axon (Peters, 1964). The ensheathment of the axons enables the speed saltatory conduction of the neuronal impulses, axon survival and maintenance (Hartline \& Colman, 2007). Myelin takes up 40-50\% of dry weight in the central nervous system. Due to its low density, it is fairly easy to fractionate it by sucrose gradient centrifugation to study separated fraction further with biochemical methods (Norton \& Poduslo, 1973). Moreover, studies of the myelin biogenesis, molecular structure and development are facilitated by possibility to cultivate primary cultures of the myelinating cells - oligodendrocytes. These cell cultures reproduce all essential morphological counterparts (processes and sheets), and express full myelin protein profile upon cultivation on the coverslips (Cole \& Vel, 1993; Chen et al., 2007).

\subsection{Myelin origin in CNS: oligodendrocytes}

The term neuroglia first was proposed by Virchow, to describe cells other than neurons (Virchow, 1854). After Cajal and Hortega developed metallic impregnation techniques for the microscopic studies, characterisation of the glial cells became easier and more rapid. The term oligodendroglia was first introduced by Hortega (Baumann \& Pham-Dinh, 2001).

Oligodendrocytes are smaller than astrocytes, have greater density of cytoplasm and nucleus; they also lack intermediate filaments and glycogen in their cytoplasm, possessing, however, a large number of microtubules in their processes (Baumann \& Pham-Dinh, 2001). Each oligodendrocyte extends many processes that upon contact with the neurons transform to the enwrapping sheath structures (Bunge, 1968). Every oligodendrocyte is capable to myelinate up to 40 different axons. Moreover, same axon may enwrap several oligodendrocytes (Baumann \& Pham-Dinh, 2001).

\subsubsection{Developmental characteristics of oligodendrocytes}

Before being able to myelinate, oligodendrocytes undergo a long developmental journey from migratory and mitotic precursors, via oligodendrocyte progenitors cells (OPC) and finally transforming into mature postmitotic myelin-producing cells. On every stage oligodendrocytes are characterised by different markers, summarised in Table 1.1.

Maturation of oligodendro- and astroglia occurs in the early postnatal embryo (Bradl \& Lassmann, 2010). Medial ganglionic eminence and anterior entopeduncular area are the origins of the first wave of oligodendrocyte precursor cells (OPC) formation in the forebrain. The second wave of OPCs derives from the lateral and/or caudal ganglionic eminences and joins the first one. Both waves populate entire embryonic telencephalon, including the cerebral cortex. The third wave comes from postnatal cortex (Kessaris et al., 2006). 


\begin{tabular}{|c|c|c|c|}
\hline Marker & Developmental stage & Comments & Reference \\
\hline A2B5 & $\mathrm{OPC}$ & $\begin{array}{l}\text { recognises gangliosides } \mathrm{GT}_{3} \\
\text { and its } O \text {-acetylated deriva- } \\
\text { tive; } \\
\text { both are downregulated in } \\
\text { the mature oligodendrocyte }\end{array}$ & $\begin{array}{l}\text { (Farrer \& Quarles, 1999; } \\
\text { Dubois et al., 1990) }\end{array}$ \\
\hline CNP & $\begin{array}{l}\text { immature and differenti- } \\
\text { ated oligodendrocytes }\end{array}$ & $\begin{array}{l}\text { highly specific, non- } \\
\text { compact myelin }\end{array}$ & (Sprinkle, 1989) \\
\hline GalC & $\begin{array}{l}\text { differentiating oligodendro- } \\
\text { cytes, mature oligodendro- } \\
\text { cytes }\end{array}$ & compact myelin & $\begin{array}{l}\text { (Baumann \& Pham-Dinh, } \\
\text { 2001) }\end{array}$ \\
\hline $\mathrm{GD}_{3}$ & $\begin{array}{l}\text { OPC and immature oligo- } \\
\text { dendrocytes }\end{array}$ & $\begin{array}{l}\text { expression disappears as } \\
\text { cell matures }\end{array}$ & $\begin{array}{l}\text { (Baumann \& Pham-Dinh, } \\
\text { 2001) }\end{array}$ \\
\hline MBP & $\begin{array}{l}\text { differentiated, mature oligo- } \\
\text { dendrocytes }\end{array}$ & compact myelin & $\begin{array}{l}\text { (Baumann \& Pham-Dinh, } \\
\text { 2001) }\end{array}$ \\
\hline MAG & $\begin{array}{l}\text { differentiated, mature oligo- } \\
\text { dendrocytes }\end{array}$ & non-compact myelin & $\begin{array}{l}\text { (Schachner \& Bartsch, } \\
2000)\end{array}$ \\
\hline MOG & $\begin{array}{l}\text { differentiated, mature oligo- } \\
\text { dendrocytes }\end{array}$ & $\begin{array}{l}\text { margins of the compact } \\
\text { myelin }\end{array}$ & $\begin{array}{l}\text { (Coffey \& McDermott, } \\
1997)\end{array}$ \\
\hline $\mathrm{O} 1$ & $\mathrm{OPC}$ & $\begin{array}{l}\text { recognises galactocerebro- } \\
\text { sides; remains present in } \\
\text { the mature state } \\
\text { and monogalactosyldiglyc- } \\
\text { erides }\end{array}$ & $\begin{array}{l}\text { (Sommer \& Schachner, } \\
\text { 1981) }\end{array}$ \\
\hline $\mathrm{O} 4$ & $\begin{array}{l}\text { OPC, decreases in mature } \\
\text { oligodendrocytes }\end{array}$ & recognises sulfoglycolipids & $\begin{array}{l}\text { (Sommer \& Schachner, } \\
1981)\end{array}$ \\
\hline PLP & $\mathrm{OPC}$ & $\begin{array}{l}\text { compact myelin; remains in } \\
\text { mature stage. }\end{array}$ & (Timsit et al., 1992) \\
\hline
\end{tabular}

Table 1.1: Markers for oligodendrocytes development.

Oligodendrocytes need to migrate a long way from their origin to enable a proper coverage of the developing brain (Frost et al., 1996). Since oligodendrocytes loose they migration ability upon maturation, it is crucial to prevent their premature differentiation. This is achieved via the Notch pathway activation (Wang et al., 1998). Several migratory cues to guide oligodendrocytes on their way are described: the chemokine CXCL1 (de Castro \& Bribián, 2005), growth factors like PDGF, FGF (Redwine et al., 1997; Spassky et al., 2001), and hepatocyte growth factor (Yan \& Rivkees, 2002); chemotropic molecules like netrins and secreted semaphorins (Jarjour et al., 2003; Tsai, 2003). However, the exact molecular mechanism still remains unknown (Bradl \& Lassmann, 2010). After reaching their targeted location, oligodendrocyte progenitors cells settle down along the nerve. At this stage cells start to express the O4 marker (Sommer \& Schachner, 1981), become less motile (Baumann \& Pham-Dinh, 2001) and loose their response to PDGF (Gao et al., 1998). They form multiple processes and are able to proliferate.

Upon acquiring galactosylceramide (GalC) and loosing the expression of GD3 
A

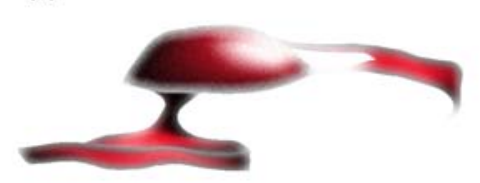

C

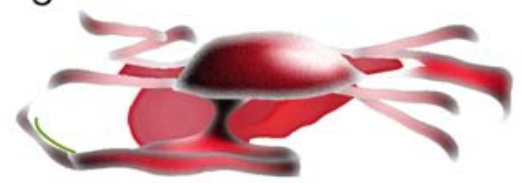

B

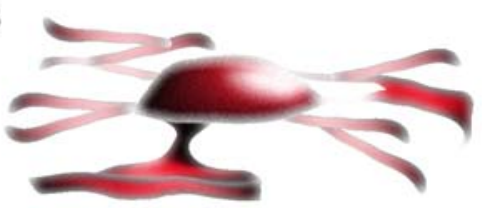

D

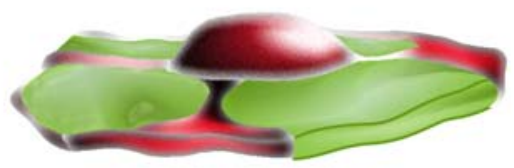

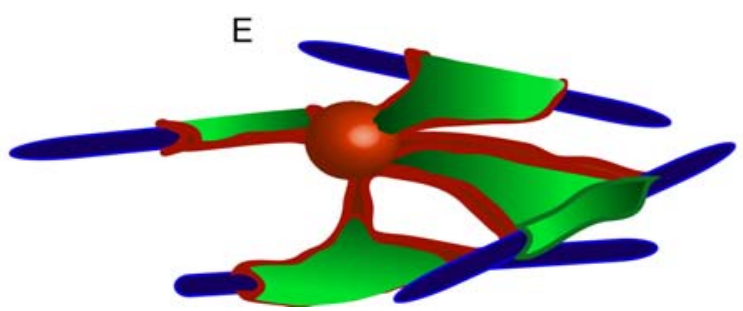

Figure 1.1: Schematic overview of the developmental stages of oligodendrocytes in culture. (A) oligodendrocyte progenitor cells: possess only several non-branched processes; (B) immature oligodendrocyte: number of processes increases and they become branched; $(\mathrm{C})$ nonmyelinating oligodendrocyte: non-compacted myelin-like sheets begin to form in between branched processes, filled with CNP (red); (D) myelinating (mature) oligodendrocyte: MBP (green) is expressed and fills the sheets, zipping them and squeezing CNP (red), along with other proteins, to the margins of the cell (processes and the rim); in this way the functional and structural polarisation is achieved; (E) co-culture with neurons (blue): onset of the proper myelination upon contact of the oligodendrocyte sheets with axons.

and A2B5 antigens on the cell surface, preoligodendrocytes become immature oligodendrocytes (Reynolds \& Wilkin, 1991). CNP is the first marker protein to be expressed at this stage (Sprinkle, 1989; Reynolds \& Wilkin, 1988). Myelin basic protein (MBP), myelin-associated glycoprotein (MAG) and proteolipid protein (PLP) follow and signify by their appearance the terminal maturation stage of oligodendrocytes. Myelination starts shortly after (Figure 1.1) (Kamholz et al., 1988; Pfeiffer et al., 1993).

The ability of oligodendrocyte progenitors to differentiate into oligodendrocytes is independent from the presence of neurons (Temple \& Raff, 1986). Cultures of oligodendrocytes, derived from the newborn (P0) mice are able to create a myelin-like membrane in the absence of axons, (Figure 3.1) (Baumann \& Pham-Dinh, 2001). Therefore, these cultures are a very useful tool to investigate myelin biogenesis and development. This structural separation of mature oligodendrocytes into compact sheets and non-compact processes leads to high functional polarisation of these cells: compact domains recapitulate myelin in vivo and non-compact processes play a trophic and supporting function (Figure 1.1). 


\subsubsection{Myelination onset}

Generation of the specific number of oligodendrocytes to successfully myelinate a given amount of axons depends on the proliferative rate of oligodendrocyte progenitors, level of programmed cell death that occurs during development and on axon-to-oligodendrocyte signalling (Barres \& Raff, 1999).

Axons with diameter above $0,2 \mu \mathrm{m}$ are selected by oligodendrocytes for the myelination event (Simons \& Trajkovic, 2006). The cues for this recognition in central nervous system (CNS) are unknown, whereas in peripheral nervous system (PNS) this signal comes from neuronal neuregulin-1 (NGR1) type III, interacting with ErbB receptors on the Schwann cells (Vartanian et al., 1997).

Electrical activity of the neurons might play a signalling role for the oligodendrocytes to start myelination in CNS. The subsequent release of ATP and adenosine due to the axonal firing can mediate neuro-glial communications. It was shown that adenosine triphosphate (ATP) can inhibit OPC proliferation and stimulate their differentiation and promote myelination (Bradl \& Lassmann, 2010).

Myelination onset has a very short starting window: within $12-18$ hours the first wrapping events should be accomplished. Therefore, oligodendrocytes are capable of the rapid synthesis of large membrane amounts, together with rapid translation and trafficking of the proteins (Cahoy et al., 2008). Several features of oligodendrocyte protein machinery were described so far. Myelin basic protein, for instance, is translated next to the myelin sheath. mRNA of MBP is transported within hnRNP granules in there directly from the pericarion. This enables fast incorporation of MBP in between the two membrane layers to zip the myelin sheath (Ainger et al., 1997). Other major component of the myelin sheath, PLP/DM20, is delivered by vesicular transport. Both processes are suggested to be under surveillance of the neuronal signals (Bradl \& Lassmann, 2010).

Almost all knowledge collected throughout decades about myelin biosynthesis and oligodendrocyte development has been elucidated using rodents (rat and mice) as model systems and further extrapolated to humans. However, there are several main differences to be mentioned and to payed attention to when using such a simplified approach (reviewed in: (Bradl \& Lassmann, 2010)):

- Difference in the time of myelination: decades in human and weeks in mice, due to the more simple organisation of the rodent.

- Even though the density of oligodendrocytes per $\mathrm{mm}^{3}$ is similar between rodents and human, rodents have far less oligodendrocytes.

- Several brain regions have a different level of development. Neocortical regions in humans are absent in mice, whereas the olfactory bulbs are underdeveloped in humans, but highly advanced in rodents.

- Certain guiding factors have a different effect on the human and rodent OPCs (Wilson et al., 2003; Zhang et al., 2000).

Nevertheless, studies of the rodent brain tissues and cultures of the primary cells from the rodent brains (neurons and glia) have led to immense insight of functioning and developing of the CNS. 


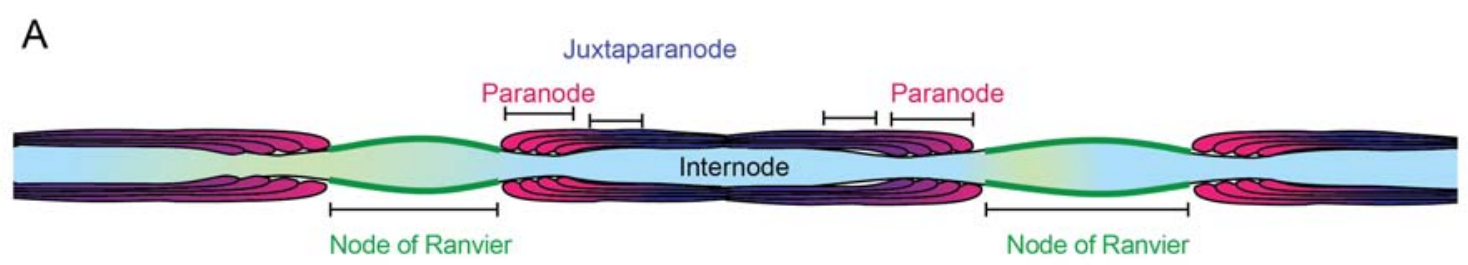

B

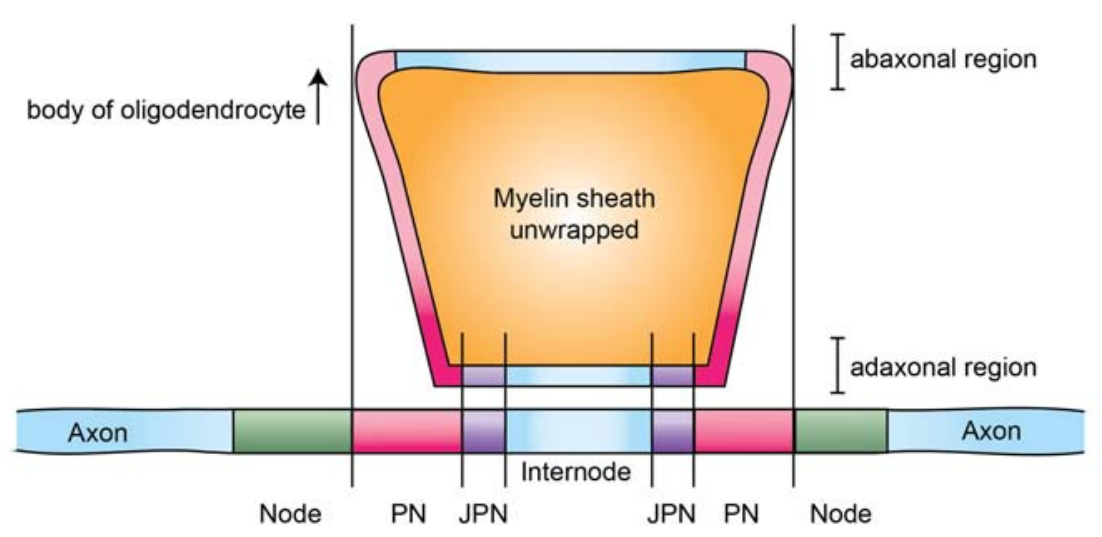

Figure 1.2: Schematic overview of the myelin architecture in vivo. (A) Longitudinal axes of the axon (blue), nodes of Ranvier (light green) and myelin domains: paranode (red, juxtaparanode (purple). (B) Myelin sheath in an unwrapped state (orange) with the corresponding domains: axon in blue, node of Ranvier in green, paranode (PN) in red, juxtaparanode in purple. Arrowed line shows direction to the oligodendrocyte body. Modified from (Salzer \& James, 2003).

Apart from the myelin ensheathment, oligodendrocytes also influence the clustering of sodium channels in the node of Ranvier (Kaplan, 1997). Moreover, oligodendrocytes influence the axonal transport and viability, since any disturbances of the myelination result in the axonal swellings, increased axonal diameter, changed rates of the axonal transport and decrease in the microtubules number and stability (Colello et al., 1994; Mason et al., 2001; Sánchez et al., 1996; Kirkpatrick et al., 2001; Dupree et al., 1999).

\subsection{Myelin structure}

In the CNS each oligodendrocytes can enwrap up to 40 axons. The regions, covered by the insulating myelin sheath are called internodes.

The adjacent internodes are separated by nodes of Ranvier - the myelin-free regions (Figure 1.2). Here the axonal membrane (axolemma) is exposed to the extracellular milieu (Bunge, 1968). These regions contain a high density of $\mathrm{Na}^{+} / \mathrm{K}^{+}$ ATPases, $\mathrm{Na}^{+} / \mathrm{Ca}^{2}+$ exchangers and sodium and potassium channels, all together allowing the generation of the action potential (Waxman \& Ritchie, 1993). The primary node, where the action potential is generated, is placed immediately after the end of axonal body and is named - axon initiation segment (AIS). Upon excitation of the membrane in this region, current can not flow through the myelin insulating sheath. Thus, depolarisation of the membrane occurs only at the adjacent node of Ranvier, leading to the speed saltatory conduction of the impulse. The term saltatory conduction (from Latin saltare - to jump, to leap, to dance) was proposed in 1952 by Hodgkin and Huxley (Hodgkin \& Huxley, 1952).

The glial cells abruptly open at the inner and outer regions around the tightly 
compacted myelin, forming less compacted tongues: inner and outer - paranodal loops (paranode) (Figure 1.2). These loops display a regular pattern on each side of the node of Ranvier, weaving spirally around an axon and retaining glial cytoplasm. Each of these paranodal loops is tightly attached to the axolemma through a septate-like junctions. Two complexes (Caspr/paranodin and contactin/F3) are highly enriched in the paranodes from the axolemma site (Menegoz et al., 1997; Rios et al., 2000), whereas glial part in this region contains a $155 \mathrm{kDa}$ isoform of neurofascin NF155. Contactin brings together two membranes via interaction with the NF155, anchored in the glia membrane and a Caspr/paranodin from the axolemma (Salzer \& James, 2003).

The small innermost region following the paranode is called juxtaparanode. Axolemma in these regions contains high number of $\mathrm{K}^{+}$-channels, whereas the glia side harbours TAG-1 (transient axonal glycoprotein-1) (Poliak \& Peles, 2003).

Adaxonal and abaxonal domains create the asymmetry along the radial axis of myelin. Adaxonal region is an inner most non-compact layer of myelin that runs parallel to the axon. It contains MAG and Necl4 (Quarles, 2007; Spiegel et al., 2007). Abaxonal region is a small cytoplasmic part on the outside of the myelin sheath. This outer most layer also lacks the compaction (Salzer \& James, 2003).

For the schematic overview of the domains localisation (Figure 1.2).

\subsection{Myelin lipid composition}

Lipids are main components of the myelin, representing $80 \%$ of its dry weight. Lipids within myelin tissues have a low turnover rate. Upon injection of radioactive cholesterol label into mouse brain, grey matter got clear of it within several weeks; radioactive signal from the myelin tissues, however, could be read after as long as a year (O’Brien, 1965b).

Main functions of the lipid molecules within the animal cell are: to store the energy (triacylglycerols only) and to structure membrane. Furthermore, several type of lipids are important signalling molecules (IP3, DAG), or they are involved in the protein tethering to the membrane (prenyl, farnesyl and other isopren residues) (Siegel, 2006). In addition, lysophospholipids and steroids modulate cellular metabolism via interaction with receptors on the endoplasmatic reticulum and in the nucleus (Bieberich, 2012).

Since myelin is deprived from triacylglycerols, storage function of the lipids in this membrane is not of a great importance (Siegel, 2006). However, almost all main classes of lipids are present within the myelin membrane: glycerolipids, cerebrosides and cholesterol (Figure 1.3). Molar ratios of these classes in different parts of the brain are summarised in Table 1.2.

Within myelin lipids play an insulating role to achieve a high resistance capacity against electric impulses and provide their speed conduction along the nerves. Moreover, there are studies claiming that myelin galactolipids (galactosylcerebroside and sulphatides) are involved in glycosynapse formation that guide a proper establishment of the paranodal-axoglial junction by acting as ligands for axolemmal proteins (Boggs et al., 2004; Boggs et al., 2010). Further functions of the myelin lipids are discussed below in the corresponding subsections. 


\begin{tabular}{lccccc}
\hline Substance & \multicolumn{2}{c}{ Myelin } & White matter & Grey matter & Whole brain \\
\cline { 2 - 3 } & Human & Rat & Human & Human & Rat \\
& & & & & \\
\hline Protein & 30.0 & 29.5 & 39.0 & 55.3 & 56.9 \\
Lipid & 70.0 & 70.5 & 54.9 & 32.7 & 37.0 \\
Cholesterol & 27.7 & 27.3 & 27.5 & 22.0 & 23.0 \\
Cerebroside & 22.7 & 23.7 & 19.8 & 5.4 & 14.6 \\
Sulfatide & 3.8 & 7.1 & 5.4 & 1.7 & 4.8 \\
Total galactolipid & 27.5 & 31.5 & 26.4 & 7.3 & 21.3 \\
Ethanolamine phosphatides & 15.6 & 16.7 & 14.9 & 22.7 & 19.8 \\
Phosphatidylcholine & 11.2 & 11.3 & 12.8 & 26.7 & 22.0 \\
Sphingomyelin & 7.9 & 3.2 & 7.7 & 6.9 & 3.8 \\
Phosphatidylserine & 4.8 & 7.0 & 7.9 & 8.7 & 7.2 \\
Phosphatidylinositol & 0.6 & 1.2 & 0.9 & 2.7 & 2.4 \\
Plasmalogens & 12.3 & 14.1 & 11.2 & 8.8 & 11.6 \\
Total phospholipid & 43.1 & 44.0 & 45.9 & 69.5 & 57.6 \\
\hline
\end{tabular}

Table 1.2: Comparison of the human and rat brain composition. Protein and lipid values are given in percentage of the dry weight; everything else in total lipid weight percentage. Modified from (Quarles, 2002).

\subsubsection{Glycerolipids and glycerophospholipids}

Glycerolipids are derivatives of the phosphatidic acid (PtdOH) and represent the main entities of the lipid bilayer of any cell. Their backbone consists of a diacylated $s n$-glycerol-3-phosphate, with $s n$ referring to stereochemical numbering. Upon esterification with fatty acids on the position $s n-1$ and $s n-2, \mathrm{PtdOH}$ yields a diacylglycerol (DAG). Fatty acids in $s n-1$ are usually saturated, whereas in $s n-2-$ unsaturated. Moreover, there are sometimes differences in the linkage of the fatty acids. There are lipid species with ether-linkage to an aliphatic alcohol, termed alkyl, or to an unsaturated alcohol, termed alk-1'-enyl. The latter lipids are referred to as plasmalogens (Siegel, 1998).

When DAG, produced from PtdOH, undergoes phosphodiester linkage in position sn-3 to a different bases (Table 1.3), it yields a broad range of the glycerophospholipids: phosphatidylethanolamine, phosphatidylcholine, phosphatidylserine, phosphatidylinositols and plasmalogens (Siegel, 1998). The amount and distribution of these lipids differ between brain, white and grey matter (Table 1.2) (Wells \& Dittmer, 1967).

Apart from phosphotidylinositols (reviewed further), phospholipids are synthesised on the cytoplasmic leaflet of the endoplasmatic reticulum (ER) and to the lesser extent in the Golgi complex and inside mitochondria (phosphatidylethanolamine). Catabolic processes of phospholipids degradation depend highly on the lipid species and are often unique, however, several catabolic pathways are common. All glycerophospholipids undergo a remodelling by phospholipase A isoform (PLA) that breaks the bonds in position $s n-1$ or $s n-2$, releasing glycerol moiety and a fatty acid. PLA1 removes 1-acyl chain and PLA2 releases 2-acyl chain from the phospholipid backbone. Phospholipase B releases both fatty acids (Bohdanowicz \& Grinstein, 2013).

The contribution of phospholipids to the cellular homeostasis and well-being is enormous. They are involved in the membrane structuring and curvature changing; signal transduction events and endocytosis; cellular growth and protein recruitment 
<smiles>[X]OCC(COP(=O)([O-])O[Y])OC([R])=O</smiles>

\begin{tabular}{lcc}
\hline $\mathbf{Y}$ & Lipid & Abbreviation \\
\hline $\mathrm{H}$ & Phosphatidate & PtdOH \\
\hline $\mathrm{CH}_{2}-\mathrm{CH}_{2}-\mathrm{N}^{\oplus} \mathrm{H}_{3}$ & Phsophatidylethanolamine & PE \\
\hline $\mathrm{CH}_{2}-\mathrm{CH}_{2}-\mathrm{N}^{\oplus}-\mathrm{CH}_{2}$ & Phosphatidylcholine & $\mathrm{PC}$ \\
\hline $\mathrm{N}^{\oplus} \mathrm{H}_{3}$ & & \\
$\mathrm{CH}_{2}-\mathrm{C}-\mathrm{COO}^{\ominus}$ & Phosphatidylserine & PS \\
$\mathrm{H}$ & & \\
$\mathrm{CH}_{2}-\mathrm{CH}-\mathrm{CH}_{2}-\mathrm{HO}$ & Phosphatidylglycerol & PtdGro \\
& & \\
\hline OH & & PtdGroPtd \\
\hline Phosphatidylglycerol (several) & Cardiolipin &
\end{tabular}

Table 1.3: Structure of glycerophospholipids. $\mathrm{X}$ and $\mathrm{Y}$ in the upper formula correspond to a different ligands, mentioned further in the table.

to the membrane. Disregulation of phospholipid metabolism may be divided into three groups, depending on the stage of the phospholipids metabolism disfunction: synthesis, distribution and catabolism. Each stage is associated with several severe disorders (Bohdanowicz \& Grinstein, 2013).

\section{Phosphatidylcholine}

Phosphatidylcholine (PC) is a zwitterionic phospholipid with a choline residue as a headgroup (Table 1.3). Apart of the choline head group, the glycerol backbone is esterified with two additional fatty acids that could be greatly variable: one being a saturated fatty acid (palmitic or hexadecanoic acid, margaric or heptadecanoic acid) and one being an unsaturated fatty acid (oleic acid, or 9Z-octadecenoic acid) (Bohdanowicz \& Grinstein, 2013).

$\mathrm{PC}$ is a major component of biological membranes, comprising $45-55 \%$ of the total lipids (Fernández-Murray, 2007). It is commonly found in the outer leaflet, as a result of aminophospholipid depletion by the flippases (Figure 1.3) (Devaux, 1992). To synthesise PC, choline phosphotransferase couples cytidine diphosphate (CDP)choline with diacylglycerol (DAG), releasing cytidine monophosphate (CMP). This is a ubiquitous pathway, however in hepatocytes an alternative pathway via three sequential methylation of phosphatidylethanolamine takes place (Cui et al., 1993).

$\mathrm{PC}$ is usually regarded as a strictly structural component of the cellular membrane. Nonetheless, it was reported to be involved in signal transduction events, serving as a substrate for the phospholipases to generate signal messengers: it is hydrolysed to release DAG, lysophosphatidylcholine, PtdOH (by phospholipase D) and arachidonic acid (by phospholipase A2). In neurons, PC can be additionally cleaved by phospholipase $\mathrm{B}$, yielding one glycerophosphocholine molecule and two free fatty acids (Fernández-Murray, 2007). 


\section{Phosphatidylethanolamine}

Phosphatidylethanolamine (PE) is a zwitterionic lipid with mostly structural function, constituting $15-25 \%$ of the phospholipids (Vance, 2008). PE is enriched in the inner membrane leaflet, due to the its active translocation across the membrane by the aminophospholipid flippases (Figure 1.3) (Balasubramanian \& Schroit, 2003). Due to a small size of the head group, PE adopts a moderately conical shape and induces a negative curvature to the membrane (Bohdanowicz \& Grinstein, 2013).

PE consists of a combination of glycerol, esterified with two fatty acids and phosphoric acid, with the phosphate group of it combined with the ethanolamine (Table 1.3). The two fatty acids may be the same, or different, and are usually in the $s n-1$ or $s n$-2 position (Balasubramanian \& Schroit, 2003). There are two main synthetic pathways fro PE. One of it is an addition of CDP-ethanolamine to diglycerides, releasing CMP. The second one is a decarboxylation of phosphatidylserine . Last one occurs mostly in the mitochondria, executed by phosphatidylserine- decarboxylase enzyme. The step of phosphatidylserine substrate translocation to the mitochondria is a rate limiting step of this reaction (Voelker, 1989).

$\mathrm{PE}$ plays an important role in the general phospholipid metabolism, being a substrate for almost all other phospholipid classes. Phosphatydilserine synthase 2 (PSS2) makes phosphatidylserine out of phosphatidylethanolamine at the ER (Voelker, 1984). Phosphatydilethanolamine also provides an ethanolamine for the protein modifications (Bohdanowicz \& Grinstein, 2013). S-Adenosyl methionine can subsequently methylate the amine of PE to yield phosphatidylcholine (Bohdanowicz \& Grinstein, 2013).

Apart of its biosynthetic role, $\mathrm{PE}$ is an important autophagy marker. It is known to target soluble adaptors (p62 and NDP52) to the phagosome membrane leading to autophagosome formation (Tanida et al., 2008).

\section{Phosphatidylserine}

Phosphatidylserine (PS) is a phospholipid, exclusively kept on the inner leaflet of cell membranes by the activity of aminophospholipid translocases (Figure 1.3). It comprises $2-10 \%$ of the total cellular phospholipid and by virtue of its phosphoserine head group is an anionic lipid (Table 1.3) (Bohdanowicz \& Grinstein, 2013).

In mammals, phosphatidylserine is produced by calcium-dependent base-exchange reactions between phosphatidylcholine or phosphatydilethanolamine with L-serine. This reaction is held out by phosphatidylserine synthases, PSS1 or PSS2 respectively, both being an integral membrane proteins of the ER membrane (Arikketh et al., 2008). As mentioned before, the major catabolic pathway of the phosphatidylserine via decarboxylation gives rise to phosphatidylethanolamine in the mitochondria (Voelker, 1989).

PS is an important signal messenger inside and outside of the cell. Upon apoptotic changes within a cell, phosphatidylserine becomes exposed to the outer membrane leaflet. A calcium-sensitive scramblases are the enzymes that trigger this swift exposure. It serves as an "eat me" signal to the macrophages and other professional and non-professional phagocytes (Li et al., 2003; Bohdanowicz \& Grinstein, 2013).

Besides, PS interacts with several protein classes. Some proteins recognise PS via their $\mathrm{C} 2$ domain in the calcium-dependent manner and get activated upon this 
recognition (Vance \& Steenbergen, 2005). Proteins with polybasic motifs in the form of an amphiphilic alpha-helixes in their structures (K-Ras, Rho GTPases, MBP) get attracted electrostatically to the membrane PS moieties (Hancock et al., 1990; Musse et al., 2008; Zhang et al., 2001).

These interactions are important for the protein targeting to the membrane and for inducing conformational changes to regulate protein function.

\section{Plasmalogens}

Plasmalogens are glycerophospholipids that possess a fatty alcohol at positions $s n-1$, instead of fatty acid. The fatty alcohol used are mostly restricted to a saturated species with C16, or saturated and mono-unsaturated C18. They ether linkages differ. It is either a modified vinyl ether bond, named 1-0-(1Z-alkenyl) that is prominent for glycerophosphoethanolamines (GPEtn), or a 1-0-alkyl bond characterised for the glycerophosphocholines (GPCho). At the $s n-2$ position, plasmalogens are enriched in polyunsaturated fatty acids, especially docosahexaenoic, or arachidonic acid (Fahy et al., 2005).

Amount of plasmalogens in the cellular membrane is about $15-20 \%$ of the total phospholipids, and more than $50 \%$ of all are glycerophosphoethanolamines (GPEtn) (Figure 1.3. Brain plasmalogens consists almost 90\% out of GPEtn and comprise $22 \%$ of the total brain lipids (Braverman \& Moser, 2012). The total amount of plasmalogens is age-dependent and increases during development and myelination (Nagan \& Zoeller, 2001). The plasmalogen synthesis occurs on the luminal side of peroxisome membrane (Brites et al., 2004) and is accomplished by 2 enzymes: glyceronephosphate $O$-acyltransferase (GNPAT) and alkylglycerone phosphate synthase (AGPS).

Grey matter plasmalogens are highly unsaturated at the $s n$-2 position that influence their bioactivity, contributing to a cell-cell communication events and facilitating membrane fusion events. The myelin plasmalogens are rather inert, due to the predominance of saturated and monounsaturated species and thus conducting a membrane structuring role (Han et al., 2001). The structural role of plasmalogens includes a maintenance of a physical bilayer properties such as phase transition temperature from gel to fluid state and packing of acyl chains and lateral domains (Farooqui et al., 2000). In diacylglycerophospholipids, the acyl chain at the $s n$-2 position creates an additional bend that increases the molecular cross sectional area. Plasmalogens lack this bend, thus their $s n-2$ and $s n-1$ chains are in a closer proximity, resulting in a decreased fluidity and an increased membrane order (Paltauf, 1994).

Another prominent feature of plasmalogens and their impact on the overall membrane stability, apart of structural, is their prompt oxidation, when exposed to the free radicals. It occurs due to a relatively low disassociation energy of the hydrogen atoms adjacent to the vinyl ether. It makes plasmalogens a sacrificial oxidant, to spare the polyunsaturated fatty acids and other vulnerable lipids. Furthermore, oxidative products of plasmalogens are unable to propagate lipid peroxidation (Sindelar et al., 1999). 

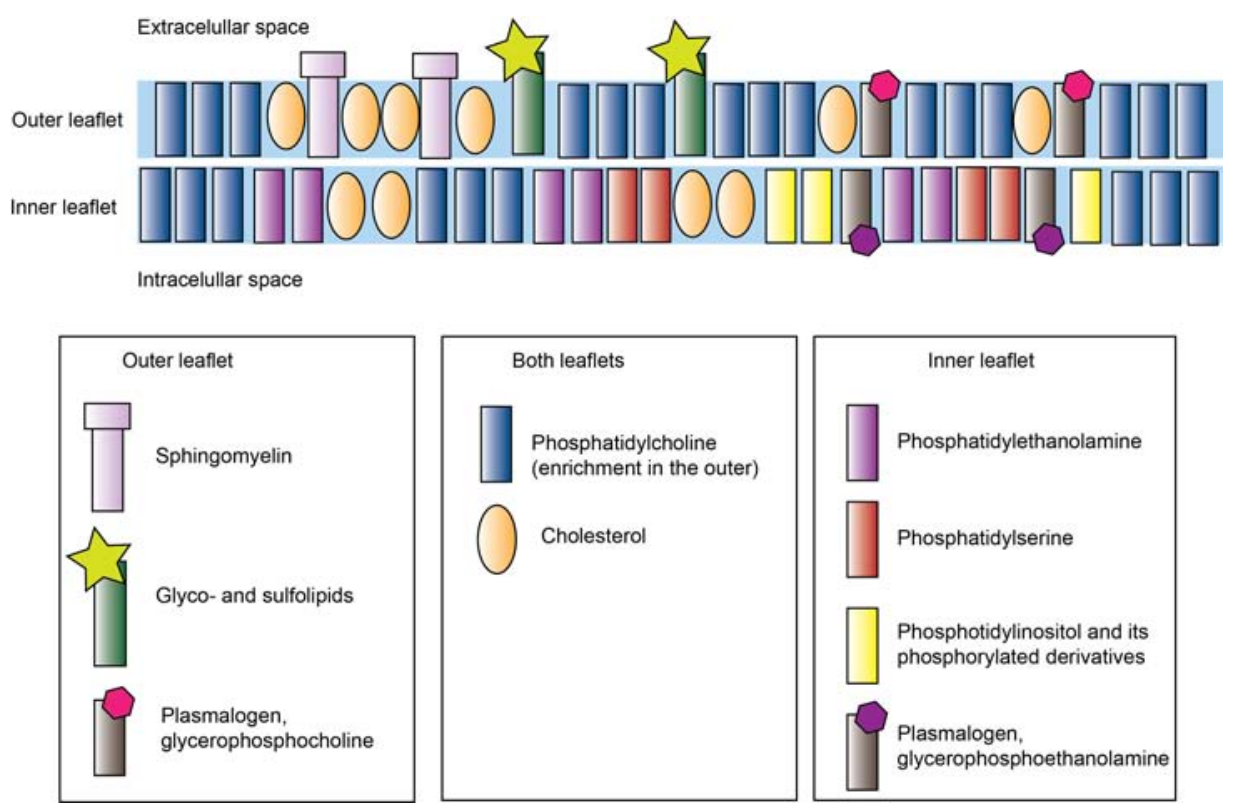

Figure 1.3: Schematic representation of the lipid distribution within outer and inner membrane leaflets. Colour-coded signs of the corresponding lipids are boxed. See text for more information.

\subsubsection{Phosphatidylinositol, PtdIns}

This anionic lipid species comprises $10-15 \%$ of the cellular phospholipids. They are present in a various membrane compartments of the cell; in the plasma membrane it is enriched in the inner leaflet (Figure 1.3) (Devaux, 1992). PtdIns possesses a unique feature of being able to undergo reversible and transient changes at its head-group: phosphorylation on position 3,4 and/or 5. This yields seven distinct phosphoinositides species, involved in the regulation of the broad spectra of the cellular metabolic pathways (Bohdanowicz \& Grinstein, 2013).

The main precursor of PtdIns synthesis is CDP-DAG. An enzyme, PtdIns synthase, is a soluble protein, associated with ER. It condensates myo-inositol and CDP-DAG to produce PtdIns (Antonsson, 1997). Secondary pathway to obtain PtdIns acts via dephosphorylation of already available phosphoinositides; for example via dephosphorylation of phosphatidyl-3,4,5-triphosphate $\left(\mathrm{PIP}_{3}\right)$ by myotubularin phosphatases (Sasaki et al., 2009).

Apart of being a precursor of the plethora of phosphoinositides species, PtdIns is also an important source of the glycosylphosphatidylinositol (GPtdIns) - an anchor for the membrane-destined proteins. The synthesis of GPtdIns occurs on the cytosolic side of ER, with subsequent flipping of the product inside the lumen, where the $\mathrm{COOH}$ terminus of the proteins can be covalently attached.

\section{Phosphatidylinositol-(4,5)-bisphosphate, $\mathrm{PIP}_{2}$}

Phosphatidylinositol-(4,5)-bis-phosphate is mostly enriched in the inner leaflet of the plasma membrane, and represents $1-2 \%$ of the total phospholipids. It is produced from PtdIns(4)P and PtdIns(3)P by specific kinases. Another pathway to acquire $\mathrm{PIP}_{2}$ is a dephosphorylation of $\mathrm{PIP}_{3}$ by a phosphatase and tensin homologue (PTEN) phosphatase. Furthermore, $\mathrm{PIP}_{2}$ itself can be dephosphorylated by a number of 5/-phosphatases (synaptojanin, OCRL, Inpp5B) (Sasaki et al., 2009). 
Moreover, $\mathrm{PIP}_{2}$ could be subjected to the cleavage by lipases to produce DAG and $\mathrm{IP}_{3}$ (a key to the $\mathrm{Ca}^{2+}$ mobilisation). Phosphorylation by kinases (PI3K) produces a $\mathrm{PIP}_{3}$ out of $\mathrm{PIP}_{2}$, a critical signal molecule in the cellular activation (Balla et al., 2009).

Apart from its precursor destiny, $\mathrm{PIP}_{2}$ is involved in the protein recruitment to the plasma membrane. Several classes of protein possess a range of $\mathrm{PIP}_{2}$-recognition domains, such as $\mathrm{PH}$ (pleckstrin homology), ETNH (epsin $\mathrm{NH}_{2}$-terminal homology), FERM (band 4.1/ezrin/radixin/moesin) and others (Lemmon, 2008) that recognise and binds specifically to the $\mathrm{PIP}_{2}$ moiety in the inner leaflet. Due to this key feature of the membrane targeting, $\mathrm{PIP}_{2}$ is involved into ample amount of cellular events (Czech, 2000; McLaughlin et al., 2002).

Functions of $\mathrm{PIP}_{2}$-targeted proteins:

- Enzyme activation: $\mathrm{PIP}_{2}$ controls the self-production by regulation via positive and negative feedback loops the functionality of PtdIns kinases and phosphatases (McLaughlin et al., 2002).

- Control of the cytoskeleton: $\mathrm{PIP}_{2}$ plays an important role in the cytoskeleton attachment to the membrane, via activation of the actin-binding proteins (Czech, 2000; Sechi \& Wehland, 2000).

- Membrane traffic: there is a plethora of $\mathrm{PIP}_{2}$-binding molecules that orchestrate exocytosis and clathrin-mediated endocytosis: AP-2 and AP180 adaptor proteins, dynamin, synaptotagmin, synaptojanin-1, to name a few (Simonsen et al., 2001; Martin, 2001).

- Regulation of ion channels: mainly via $\mathrm{IP}_{3}$ and DAG production from phosphatidyl-(4,5)-bisphosphate (Haugh et al., 2000).

- Binding of scaffolding proteins: $\mathrm{PIP}_{2}$-recognition domains, such as $\mathrm{PH}$ (pleckstrin homology), ETNH (epsin $\mathrm{NH}_{2}$-terminal homology), FERM (band 4.1/ezrin/radixin/ moesin) serve as an anchoring point for the scaffolding proteins (McLaughlin et al., 2002; Lemmon, 2008).

$\mathrm{PIP}_{2}$ has a unique feature to keep its negative charges at a normal $\mathrm{pH}$, contributing to the overall electronegativity of the inner leaflet of the plasmalemma. Negatively charged domains of $\mathrm{PIP}_{2}$ create binding platforms for the positively charged proteins. One family of the $\mathrm{PIP}_{2}$-sequestering proteins are MARCKS (myristoylated alanine-rich $\mathrm{C}$ kinase substrate) proteins. They are intrinsically disordered, membrane-bound via a lipid anchor and possess a polybasic domain in the middle. This domain consists of 13 basic residues (Wang et al., 2001) and bind preferentially to $\mathrm{PIP}_{2}$, however PIP 3 binding is also not excluded (McLaughlin et al., 2002). These interactions have a dual significance: MARCKS proteins get targeted and anchored into the plasma membrane, and, subsequently, $\mathrm{PIP}_{2}$ moiety gets sequestered within this regions. Release of these interactions leads to a swift increase in the pool of $\mathrm{PIP}_{2}$, upon necessity. Local increase of actin due to the release of the $\mathrm{PIP}_{2}$ from this sequestration might also take place (Bohdanowicz \& Grinstein, 2013). 


\section{$\mathrm{PIP}_{2}$ as a general actin modulator}

A tremendous impact $\mathrm{PIP}_{2}$ displays on the actin cytoskeleton. It achieves this upon interaction with a plethora of proteins that exhibit actin-regulation activity. $\mathrm{PIP}_{2}$ mostly promotes actin polymerisation scenario via aiding in the dissociation of globular $(\mathrm{G})$-actin from the filament-severing and actin-monomer-binding proteins (profilin, gelsolin, villin, severin, other Cap proteins) (Lassing \& Lindberg, 1988; Machesky \& Hall, 1997). Most of the $\mathrm{PIP}_{2}$-binding peptides possess a positively charged residues clusters or specific PH-domains (Sechi \& Wehland, 2000).

$\mathrm{PIP}_{2}$ influence on actin is also modulated via Rho-family of small GTPases: Rho, Rac and Cdc42 (Schmidt \& Hall, 1998). Rho-GTPase, for instance, activates a phosphatidyl 4-phosphate 5-kinase (PtdIns(4)P 5-kinase) leading to an increase in $\mathrm{PIP}_{2}$ levels (Hartwig \& Kwiatkowski, 1991). Similar role has been described for the Rac-GTPase, after a specific Rac-activated PtdIns(4)P 5-kinase has been identified (Tolias et al., 1998). PIP 2 was shown to be a co-activator of the WASP family proteins, acting via Cdc42 that favours the nucleation of actin polymerisation through the Arp2/3 complex (Fawcett \& Pawson, 2000).

Moreover, another class of small GTPases, the ADP-ribosylation factor (ARF) proteins, has recently been described to be involved in the $\mathrm{PIP}_{2}$ production. This class of proteins generally participates in the membrane traffic regulation, membrane ruffling and cell spreading. ARF6 co-localises with Rac at the plasma membrane and is involved in the clathrin-mediated endocytosis and Fc-dependent phagocytosis (Donaldson \& Jackson, 2000). ARFs were shown to co-localise and further activate type $\alpha$ PtdIns(4)P 5-kinase at the plasma membrane with the subsequent increase in the $\mathrm{PIP}_{2}$ production and cytoskeleton remodelling (Honda et al., 1999).

Implementations of the $\mathrm{PIP}_{2}$-specific $\mathrm{PH}$ domains, fused to GFP, revealed that almost all $\mathrm{PIP}_{2}$-specific markers were targeted to the cellular plasma membrane and not to the internal membrane (ER, Golgi or nuclear). The same is true for the cytoskeleton structures: their assembly always occurred at the plasma membrane. This was even further illustrated by the studies of the actin nucleation sites that are essentially all located just beneath the plasma membrane. New actin monomers were added between plasma membrane and the actin nucleation sites, whereas actin disassembly occurred within the cell body (Insall \& Weiner, 2001).

Another feature of the actin assembly is an outward alignment of the filaments near the plasmalemma. Cell movements occur by intercalating new monomers between plasma membrane and the existing actin cortex and thus pushing the membrane forward. This requires a specific orientation of the growing, barbed, ends of the actin filaments. Therefore some signalling mechanism must exist to regulate a positioning of a growth of new actin filaments. $\mathrm{PIP}_{2}$ might regulate this via interactions with the actin-capping proteins. These proteins bind to the barbed ends of the filament and prevent further growth. $\mathrm{PIP}_{2}$, in turn, can displace them, enabling elongation, but only if the direction of the growth is a proper one (Insall \& Weiner, 2001).

Furthermore, all described mutual activations of actin-binding proteins also occur only at the plasma membrane. Taking into account that $\mathrm{PIP}_{2}$ levels are relatively high in the cell, it has been suggested that $\mathrm{PIP}_{2}$ impact on the actin regulation results mainly in the general localisation of the actin assembly to the plasma membrane and the proper directionality of this assembly, rather than local increase in the actin 
levels (Insall \& Weiner, 2001).

\section{Phosphatidylinositol-(3,4,5)-triphosphate, $\mathrm{PIP}_{3}$}

Amount of $\mathrm{PIP}_{3}$ in the resting cell is scarce, and even upon activating signals it does not rise more than $1 \%$ of the total lipid amounts (Stephens et al., 1993). $\mathrm{PIP}_{3}$ is a major signalling molecule within a plasmalemma and a potent growth and survival signal (Bohdanowicz \& Grinstein, 2013). Phosphatidylinositol-(3,4,5)-triphosphate is mostly synthesised from phosphatidylinositol-(4,5)-bisphosphate due to activity of class I membrane PI3-kinases (Engelman, 2009). The breakdown of $\mathrm{PIP}_{3}$ occurs via its phosphorylation by 3/-phosphatases (PTEN) and 5/-phosphatases (SHIP I and SHIP II) (Sasaki et al., 2009; Liu \& Casaccia, 2010). Failure in at least one of the enzymes, controlling $\mathrm{PIP}_{3}$ turnover is sufficient to induce tumorogenesis in mice (Kinross et al., 2012) and a broad range of mutations in PI3K and PTEN are reported to be often found in the cases of human cancer (Chalhoub \& Baker, 2009).

Despite its high negative charge, $\mathrm{PIP}_{3}$ does not largely contribute to the overall charge of the inner plasma membrane leaflet, due to its low abundance. In contrary, main function of the $\mathrm{PIP}_{3}$ in the membrane is to recruit an ample variety of proteins to the place of their action. These proteins usually possess a specific type of $\mathrm{PH}$ (pleckstrin homology) or PX (phagocyte oxidase homology) domains (Scheffzek \& Welti, 2012; Wishart et al., 2001) required for the $\mathrm{PIP}_{3}$ recognition on the membrane. Among these proteins are PLC- $\gamma$, Akt, Vav, PDK isoforms (Bohdanowicz \& Grinstein, 2013).

\section{$\mathrm{PIP}_{3}$ as local actin assembly regulator}

$\mathrm{PIP}_{3}$ is a potent second messenger and actin polymerisation is a primary respond within the cell when the $\mathrm{PIP}_{3}$ levels get elevated (Stephens et al., 1991). Broad range of the pharmacological agents, together with mutant receptors, used to inhibit PI3-kinases have been found to interfere with stimulus-induced actin polymerisation in cells, including neutrophils (Niggli \& Keller, 1997) and fibroblasts (Wennström et al., 1994). Even though $\mathrm{PIP}_{3}$ is not always required for the actin polymerisation (Kovacsovics et al., 1995), production of this lipid alone is sufficient to burst actin nucleation in most of the cell types. It is consistent with the artificial increase of the $\mathrm{PIP}_{3}$ amount either via exogenous delivery of this lipid or via expression of the dominant-positive versions of the PI3-kinase (Derman et al., 1997; Niggli, 2000), resulting in the actin rearrangements. Coupling of $\mathrm{PIP}_{3}$-signalling to the actin polymerisation is suggested to be via guanine nucleotide exchange factors (GEFs) involved in the activation of Rho family proteins: Cdc42 and Rac, since GEF possess a $\mathrm{PIP}_{3^{-}}$ specific $\mathrm{PH}$-domain in their structure. When $\mathrm{PIP}_{3}$ increase was blocked pharmacologically, Cdc42 and Rac activation in neutrophils and Rac activation in fibroblasts were inhibited (Hawkins et al., 1995). Cdc42 stimulates actin polymerisation though the Wiskott-Aldrich Syndrome protein (WASP) and its relatives. They cause actin polymerisation by activating the Arp2/3 complex (Machesky et al., 1999).

Intriguingly, in other studies, similar pathways were described as being a $\mathrm{PIP}_{2^{-}}$ dependent. This issue brought up a speculation that even though $\mathrm{PIP}_{2}$ and $\mathrm{PIP}_{3}$ orchestrate their stimuli via common pathways, the outcome is different. The common persistent theory in the field is: $\mathrm{PIP}_{3}$ plays a role of the switch to turn on local swift 
polymerisation of actin filaments in respond to the inner and outer cues, whereas $\mathrm{PIP}_{2}$ is a marker that ensures filaments to grow at the right site and in the right direction for the constant support of the cellular shape and trafficking. Both signals are required for proper actin regulation, and they often share regulatory machinery, but their spacial and temporal activation is the ultimate determinant where, when and why actin polymerisation is due to take place (Insall \& Weiner, 2001).

\subsubsection{Sphingolipids}

The backbone of these class of lipids is build up from a ceramide and not glycerol. This backbone consists of a sphingoid base, amide-linked through its amino group to a fatty acid. To synthesise ceramide de novo, first the condensation of L-serine and palmitoyl-CoA to form 3-ketosphinganine occurs, driven by serine palmitoyltransferase. This product is then reduced, resulting in dihydrosphingosine that undergoes $\mathrm{N}$-acylation by ceramide synthase to yield a ceramide. These reactions take place on the cytosolic side of the endoplasmatic reticulum (ER) (van Meer \& Lisman, 2002).

The most prominent representatives of sphingolipids in myelin are glycosphingolipids and sphingomyelin (Table 1.4).

\section{Glycosphingolipids}

This subclass of sphingolipids is build by a ceramide backbone with an added sugar headgroup. Hydrophobic ceramide is inserted in a membrane, whereas a sugar moiety faces the extracellular space (Siegel, 1998).

The biosynthesis of glycosphingolipids continues from the acquirement of a ceramides on the cytosolic face of the ER. Ceramide can spontaneously cross ER membrane and be converted to galactosylceramide (GalC) within ER lumen. This occurs due to the transfer of a galactose residue ( $\mathrm{Gal}$ ) from the uridine diphosphate (UDP)Gal to the ceramide by UDP-Gal:ceramide galactosyltransferase (CGT) (Schulte \& Stoffel, 1993; Morell \& Radin, 1969).

The sugar residues could also consist of glucose, received from UDP-glucose that would yield glycosphingolipids (Radin, 1984). Glycosphingolipids are precursors of two subclasses of sphingolipids: globosides - neutral sphingolipids and gangliosides - acidic sphingolipids. In short: globosides contain more than one sugar: a combination of D-glucose, D-galactose and N-acetylgalactosamine. Gangliosides, in addition, have several residues of the n-acetylneuraminic (sialic) acids (NANA) bound to the sugar moieties. More than 60 gangliosides are described so far, all differing mainly in the amount and position of the NANA molecules (Siegel, 2006).

Gangliosides are ubiquitously distributed over all organism, however their concentration in brain far more exceeds that one in other tissues (Farrer \& Quarles, 1999).

Interestingly, to assess any kind of the phenotype by a knocking out the ganglioside pathway, one should perform a double knock-out, since a high level of the redundancy between gangliosides is observed and a single knock-outs do not yield a decent phenotypes (Yamashita et al., 2005). Mice devoid from any of ganglio-series develop severe neurodegeneration and dye shortly after weaning. Investigation of the brain phenotype of these mice suggested a role of gangliosides in the axon-glia attachment sites in the node of Ranvier with MAG protein being a possible interaction 


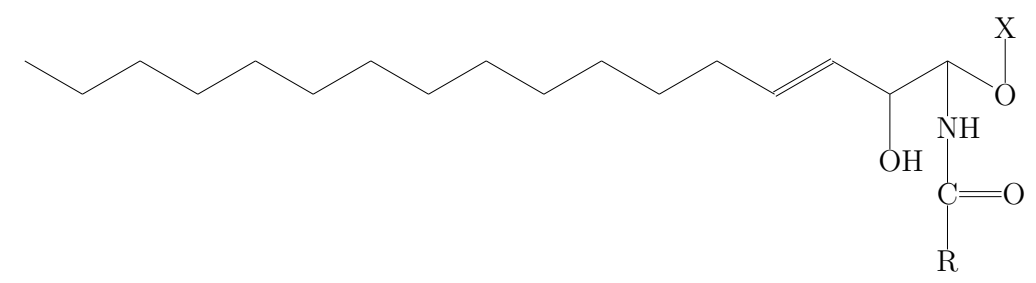

\begin{tabular}{lcc}
\hline $\mathbf{X}$ & Lipid & Abbreviation \\
\hline $\mathrm{H}$ & Ceramide & Cer \\
\hline
\end{tabular}

\begin{tabular}{lcl}
$\stackrel{\mathrm{O}}{\mathrm{I}} \mathrm{P}-\mathrm{O}-\mathrm{CH}_{2}-\mathrm{CH}_{2}-\mathrm{N}^{\oplus}-\left(\mathrm{CH}_{3}\right)_{3}$ & Sphingomyelin & $\mathrm{SM}$ \\
$\mathrm{O}$ & & \\
\hline galactose & Galactocerebroside & GalC \\
\hline glucose & Glucocerebroside & GlcC \\
\hline Lactose (Glc-Gal) & Lactosylceramide & LacC \\
\hline
\end{tabular}

Table 1.4: Structure of sphingolipids. $\mathrm{X}$ in the upper formula corresponds to a different ligands, found in the table.

partner (Chiavegatto et al., 2000).

Sulfatide version of galactosylceramide ( $\mathrm{GalC}$ ) is acquired by addition of a sulphate molecule from 3/phosphoadenosine-5/phosphosulphate to the galactose of GalC in the lumen of the Golgi apparatus (Coetzee et al., 1998).

\section{Sphingomyelin, SM}

Ceramide backbone and a phosphocholine head group are the constituents of the sphingomyelin. It is synthesised in the ER and its concentration increases, starting from ER, through Golgi and reaching its highest in the outer leaflets of the plasma membrane(Figure 1.3). Sm is mostly responsible for membrane stability However, there are some studies, revealing it presence in the inner leaflet as well (Linardic \& Hannun, 1994). The hydrophobic part of this lipid is much more saturated than in phospholipids, plus its melting temperature is higher, nearing $37^{\circ} \mathrm{C}$ (Bohdanowicz \& Grinstein, 2013). They also tend to self-aggregate and form a separate phase that features decreased fluidity properties, comparing to a glycerol-based phospholipids of the overall membranes. It makes sphingolipids one of the main constituent of so called 'lipid rafts' (van Meer \& Lisman, 2002).

Sphingomyelin is mostly found together with cholesterol in the parts of membrane that together forms detergent-resistance domains upon extraction. Here SM is involved in plasma membrane signal transduction, cholesterol efflux and intracellular lipid and protein trafficking (Rietveld \& Simons, 1998; Brown \& London, 2000). To synthesize sphingomyelin, an enzymatic transfer of the phosphocholine from phosphatidylcholine to a ceramide should occur. It takes place in Golgi apparatus. Sphingomyelin synthase is responsible for this step, yielding diacylglycerol as a byproduct of the reaction (Zhang et al., 2006).

Sphingomyelin is involved in several signal transduction pathways. Starting from a notion that upon its synthesis, diacylglycerol - one of the major signal lipids in the cellular system - is released. In contrary, the breakdown of sphingomyelin yields ceramide that is as well involved in the signalling (Kolesnick, 1994). It is, for example, highly produced in several conditions leading to apoptosis, suggesting sphin- 
gomyelin to play an important role in a cellular decision to leave or to die (Green, 2000). A more peaceful role sphingomyelin has as a structural agent, forming ordered lipid phase together with cholesterol and glycolipids among a liquid disordered phase of phosphoglycerolipids, increasing membrane rigidity and stability (Massey, 2001). The increase in membrane rigidity is achieved in part due to the higher melting temperature of sphingolipids as well as the interactions of these lipids with cholesterol. Cholesterol is a relatively small, nonpolar molecule, thus it can fill the space between the sphingolipids that increases the rigidity of the membrane (Massey, 2001).

Malfunctions of the sphingomyelin metabolism are known to trigger several severe diseases. One that is relevant to a nervous system is multiple sclerosis (MS). The general upregulation of cytokines, especially tumour necrosis factor alpha, in the cerebral-spinal fluid (CSF) of MS patients, activates sphingomyelinase. This results in a sphingomyelin breakdown, high ceramide production and increase in the apoptotic events (Jana \& Pahan, 2010).

\subsubsection{Cholesterol}

This steroid species comprises almost $23 \%$ of the body sterol pool (Figure 1.3) (Dietschy \& Turley, 2004). Moreover, 70\% of brain cholesterol is compressed within a myelin sheaths (Saher et al., 2005). The highest synthetic rate of the cholesterol in oligodendrocytes is during active myelination with a subsequent decrease after its completion (Dietschy \& Turley, 2004). A low rate synthesis still remains in the astrocytes in the adolescence (Nieweg et al., 2009). The steady high amount of cholesterol within brain is sustained either by external delivery via circulating lipoproteins or via endogenous de novo synthesis. The last one includes over 30 different enzyme and a crucial rate-limiting step, controlled by 3-hydroxy-3-methylglutaryl-CoA (HMG-CoA) reductase (Dietschy \& Turley, 2004). Even though it was shown that de novo synthesis of cholesterol in the brain is 20-80 fold lower in comparison to a liver (Spady \& Dietschy, 1983), it is the only source of cholesterol accretion, since no penetration of the blood-brain barrier by plasma lipoproteins could be demonstrated (Dietschy \& Turley, 2004).

Strikingly, disruption of the cholesterol de novo synthesis in oligodendrocytes resulted in only $30 \%$ decrease of a cholesterol amount in wild type myelin. It might be due to the fact that oligodendrocytes can start a cholesterol uptake for myelin synthesis, or receive this compound from the neighbouring glia cells (astrocytes or microglia) (Saher et al., 2005). The half-life of the cholesterol molecules in the brain is remarkably long (4-6 months in rodents and up to 5 years in humans) (Dietschy $\&$ Turley, 2004). Low rate of the cholesterol synthesis in the adulthood and absence of the cholesterol degradation in the CNS contribute to its high stability. However, a small fraction $(0.02-0.4 \%)$ of the cholesterol pool is nevertheless excreted from the brain every day (Dietschy \& Turley, 2004). The major mechanism involves an enzyme 24-hydroxylase (CYP46) that is expressed in a subset of neurons. 24hydroxycholesterol, a product of 24-hydroxylase, can cross the blood-brain barrier and enter the peripheral circulation (Russell et al., 2009).

Cholesterol is of a great importance to the myelin biogenesis, since mice with cholesterol-pathway failure (squalene synthase knock-out) showed myelination deficiencies, ataxia and tremor (Saher et al., 2005). Common neurodegenerative disorders, such as Alzheimer, Parkinson, Huntington diseases, also are characterise by 
a disregulation of cholesterol metabolism. However, there is so far no direct link to explain whether cholesterol metabolism abnormality is a primary cause or the consequence of the neurodegeneration (Vance, 2012).

\subsubsection{Fatty acid composition of myelin lipids}

Fatty acids in the nervous tissue occur mainly as a $O$-acyl groups of the glycerolipids, or as $N$-acyl groups of sphingolipids. Small amounts also are found in the free or unesterified form. Chain length of the fatty acids varies from 14 to 24 carbon atoms and contain from one to six double bonds. However, some minor fatty acids with up to 35 carbon atoms were also reported. Generally, fatty acids of the nervous tissue may be divided into four groups: saturated, unsaturated, saturated hydroxy and unsaturated hydroxy acids (Siegel, 1998).

Synthesis of the fatty acids can occur de novo in the brain, but the uptake from the blood also takes place. The last one is of great importance for the essential fatty

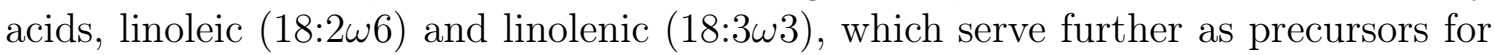

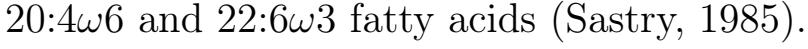

The work of O'Brien, gives a comprehensive overview about the discrepancies in the fatty acid profiles among the brain lipids. According to this study, phosphatidylethanolamine and phosphatidylserine are enriched in the 20- and 22-carbon polyunsaturated fatty acids, the major one among them - 20:4, 22:5w6 and 22:6, whereas phosphatidylcholine contained very small amount of this fatty acids (O'Brien, 1965a).

From the unsubstituted fatty acids, a broad range is present in the sphingolipids, from 14 to 26 carbon atoms, including odd-chain fatty acids, saturated and monounsaturated derivatives (Norman, 1961; O'Brien, 1965a). In general they could be subdivided into two groups: medium-chain fatty acids (14-18 carbon atoms) and the long-chain fatty acids (19-26). Grey matter has mostly medium-chain, while myelin and white matter consist of a long-chain species with a very large (up to 9fold) quantitative differences. Interestingly, in humans, fatty acid content of the grey matter sphingolipids changed throughout the life time, with a tendency to increase the length of the carbon chain with age. However, myelin sphingolipids contained the large proportion of the long chain fatty acids very early in development (10 months) (O’Brien et al., 1965).

These characteristics of the myelin lipids are thought to contribute to myelin membrane organisation and its extreme stability, since more saturated hydrocarbon chains are packed tighter in a membrane than polyunsaturated ones. Their configuration and longer hydrocarbon chains provide stronger hydrophobic interactions between the lipids with longer-chain fatty acids, in comparison to the shorter ones (O’Brien, 1965b). 


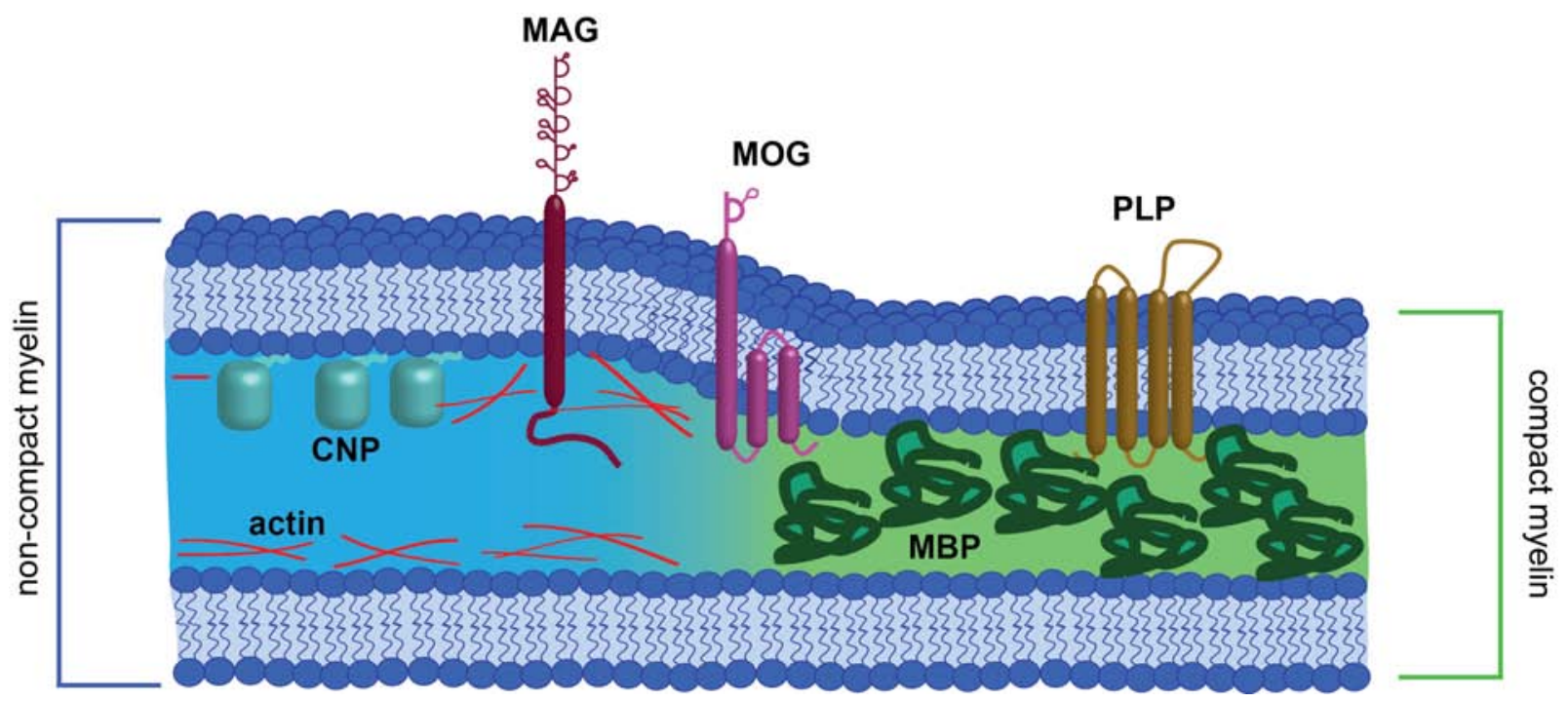

Figure 1.4: Schematic overview of the protein distribution in a myelin sheath. Noncompact regions marked with blue, compact regions shown in green. Most prominent myelin proteins are depicted.

\subsection{Myelin protein composition}

\subsubsection{Myelin basic protein, MBP}

Myelin basic protein is the most abundant protein species within the myelin sheath, corresponding to a $30 \%$ of all proteins (Baumann \& Pham-Dinh, 2001). MBP belongs to the group of intrinsically unstructured (or "natively unfolded") proteins and bears a large overall positive charge, due to a high amount of positive polar amino acids (Lysine and Arginine). Since MBP is unstructured in the solution, that makes it impossible to resolve its proper structure. It folds to achieve a conformation only upon interaction with lipids (Harauz et al., 2009). Localisation of MBP, revealed by electron microscopy, is restricted to the major dense lines, thus to the compact region of the myelin. This localisation led to a suggestion that MBP functions as glue between two adjacent membranes in vivo (Figure 1.4) (Harauz et al., 2009).

Seven exons of the MBP gene are found within a larger gene complex, termed Golli (Genes of OLigodendrocyte Lineage), that produces a number of alternatively spliced transcripts. This results in several protein isoforms for MBP: 21.5, 20.2, 18.5 and $17.2 \mathrm{kDa}$ in human, and 21.5, 20.2, 18.5, 17.24, 17.22 and $14 \mathrm{kDa}$ in mouse (Harauz et al., 2004; de Ferra et al., 1985; Kamholz et al., 1988). The major MBP isoform in human CNS is $18.5 \mathrm{kDa}$ and in mice - $14 \mathrm{kDa}$ (Baumann \& Pham-Dinh, 2001). Post-translational modifications of MBP create even further diversity of MBP. The major modification occurring at various amino acid residues throughout the peptide chain are:

- Ser/Thr phosphorylation (Chou et al., 1976),

- deimination of Arginine to Citrulline (citrullination) (Wood \& Moscarello, 1989),

- deamidation (Harauz et al., 2004), 
- acylation of N-terminal Alanine (Costentino et al., 1994),

- ADP-ribosylation (Moscarello et al., 1994),

- GTP-binding (Chan et al., 1988).

This broad variety creates the following charged isoforms MBP: C1, C2, C3, C4, C5, C6 and C8, with C1 being the least modified and most highly positively charged form and $\mathrm{C} 8$ the least positively charged and the most modified one (Boggs, 2006). These modifications occur naturally at the physiological conditions, however, they might also have a pathophysiological background. For example, phosphorylated MBP isoforms are known to have a higher turn-over, suggesting this modification to be involved in the dynamic structuring and clearance of MBP (des Jardins \& Morell, 1983). Moreover, phosphorylation of MBP changes in response to the action potential of neurons (Murray \& Steck, 1984; Atkins et al., 1997).

A primary role of MBP was considered to be an adhesion of two adjacent lipid membranes of the myelin sheath to create a compacted structure, process referred to as a zipping (Omlin et al., 1982; Smith, 1992; Riccio et al., 2000). Using fluorescent dye - Laurdan - it was demonstrated by Fitzner et al. that MBP increases the lipid order and is required for the condensation of the myelin lipid bilayer in oligodendrocytes (Fitzner et al., 2006). Therefore, an additional function of MBP was suggested, namely, an active enrichment of myelin membrane with lipids and their reorganisation within the sheath (Fitzner et al., 2006; Rosetti \& Maggio, 2007; $\mathrm{Hu} \&$ Israelachvili, 2008). More recently it was reported that MBP functions not only as a myelin-forming 'glue', but an actual size-limiting sieve, forming a barrier that restricts the diffusion of proteins with large cytoplasmic domains from one noncompact region to the myelin sheath. It was demonstrated that MBP undergoes a self-assembly, resulting in a protein meshwork that creates this molecular sieve. Moreover, MBP was shown to exhibit an active extruding properties to filter out the cytosolic proteins from the myelin sheath region to generate a lipid-rich membrane (Figure 1.4) (Aggarwal et al., 2011).

MBP interactions with lipids have been studied with a plethora of different methods: differential scanning calorimetry, use of lipid probes, small angle X-ray and neutron scattering, atomic force microscopy and spectroscopy, and miscellaneous other biophysical techniques (Hu et al., 2004; Rispoli et al., 2007; Min et al., 2009). They revealed MBP interactions with lipid membranes to be a combination of electrostatic and hydrophobic interactions.

Bearing a high positive charge, MBP interacts primarily with negatively charged lipids. It was shown that MBP laterally sequesters $\mathrm{PIP}_{2}$ in the large unilamellar vesicles in vitro and in the cellular environment (Musse et al., 2008) via non-specific electrostatic bonding. Interactions between MBP and cytoplasmic site of the membrane could be disrupted by $\mathrm{Ca}^{2+}$ influx (Nawaz et al., 2009). Thus, MBP was suggested to be a part of other class of intrinsically unstructured proteins of a similar characteristics - MARCKS (myristoilated alanine-rich C kinase substrate) - already mentioned in the text (McLaughlin et al., 2002). However, unlike MARCKS that have their membrane-interacting domain concentrated within a particular clusterforming region, MBP has its basic residues evenly distributed over its surface. This feature might be a key to the explanation of how MBP fulfils its function of bringing two opposing membrane of the myelin sheath together. Additional formation of 
the hydrogen bonds with phospholipid headgroups might also contribute to the zipping (Harauz et al., 2009). Moreover, cellular investigations revealed that MARCKS proteins can affect spatial distribution of $\mathrm{PIP}_{2}$ at the plasma membrane and create cholesterol-dependent $\mathrm{PIP}_{2}$-microdomains, function that now is also proposed to be a feature of MBP (Musse et al., 2008; Harauz et al., 2009). The cellular outcome from the formation of these $\mathrm{PIP}_{2}$-microdomains is actin accumulation, and possible recruitment of actin-binding proteins.

MBP-mediated adhesion of two membrane leaflets falls under several other regulatory mechanisms. Variation in the local $\mathrm{pH}$ and ionic strength occurring in vivo may alter the repulsion between negatively charged membrane leaflets and thus contribute to the MBP's adhesive strength (Ro \& Carson, 2004). Unique lipid composition of the myelin sheath might also affect binding of MBP: it was also suggested that high amount of cholesterol and sphingomyelin may contribute to the strength of MBP/lipids interactions (Rivas et al., 1998; Surewicz et al., 1986; Rivas et al., 1997; Epand \& Moscarello, 1982). General influence of the cholesterol and sphingomyelin amount and distribution in the cellular membrane on the lipid-protein interactions is discussed elsewhere (Maxfield \& Tabas, 2005; Gorbenko \& Kinnunen, 2006).

Mechanism of MBP translation ensures that MBP exerts its adhesive action to the negatively charged lipid in the proper place in the cell. Its translation occurs locally, precisely on the border of the processes/sheets regions, where mRNAs of MBP are transported via vesicular transport in form of the mRNA granules (Barbarese et al., 2000).

The overall importance of MBP in the CNS myelin formation and compaction is perfectly illustrated by a severe phenotype of the shiverer mice. In this naturally occurring mutants, the large portion of MBP gene is deleted (Chernoff, 1981). Most compact myelin in CNS (but not in PNS) is not presented upon this mutation. CNS myelin lamellae are poorly formed and major dense line is absent. Shiverer mice suffer from severe hypomyelination, tremor, seizures and early death (Chernoff, 1981). These effects emphasise the role of MBP in the CNS myelin compaction (Privat et al., 1979; Mikoshiba et al., 1981).

\subsubsection{2/,3/-cyclic-nucleotide phosphodiesterase, CNP}

CNP comprises $4 \%$ of the myelin protein amount and has a molecular weight of $48 \mathrm{kDa}$. Localisation of the CNP is restricted to the non-compact regions of the myelin (Figure 1.4) (Baumann \& Pham-Dinh, 2001). CNP contains a catalytic Cterminal domain with phoshodiesterase activity and N-terminal domain with distinct relation to the P-loop, containing nucleoside triphosphate hydrolases (Kursula, 2001; Stingo et al., 2007). The very C-terminus is linked to the plasma membrane via an isoprenilated region. Catalytic C-terminus classifies CNP into the family of $2 \mathrm{H}$ phosphodiesterases (Mazumder et al., 2002) and both crystal and NMR structures were revealed only for this region (Kozlov et al., 2003; Sakamoto et al., 2005), while the whole structure is yet to be determined. CNP enzymatic activity resides in the phosphodiester hydrolysis of 2/,3/-cyclic nucleotides to 2/-nucleotides (Drummond, 1962).

CNP knock-out mice suffer from progressive axonal degeneration (Lappe-Siefke et al., 2003). Interestingly, the phenotype of mice overexpressing CNP was similar to the aged rhesus-monkeys, hinting on role of CNP in the age-dependent myelin 
disruption (Gravel et al., 1996).

Despite being well-characterised, the physiological relevance of CNP still remains enigmatic, since its substrate has not been found in the brain. Several main properties are described for CNP apart from its main enzymatic activity, such as: membrane attachment (Braun et al., 1991); cytoskeleton interactions (Bifulco et al., 2002; Angelis \& Braun, 1996; Lee et al., 2005) and RNA binding (Gravel et al., 1996). Based on these findings, the role of CNP as regulator of RNA trafficking, splicing and metabolism, as well as oligodendrocyte maturation and myelin gene expression was proposed (Myllykoski et al., 2012).

It was suggested that catalytic mechanism of CNP is similar to that of RNaseA (Sakamoto et al., 2005). Thus, CNP might be involved in the RNA metabolism regulation, taking into account that some proteins in myelin (MBP for instance), are synthesised "on spot" in the processes, rather than in the cell body, after their mRNA is delivered there (Carson et al., 1998; Ainger et al., 1997). Moreover, N-terminal domain of CNP was shown to have a high structural homology to the N-terminal kinase domain of $\mathrm{T} 4$ polynucleotide kinase involved in the RNA repair (Myllykoski et al., 2012).

\subsubsection{Proteolipid protein, PLP}

PLP represents the most abundant protein in the myelin compact regions (Figure 1.4). It is a 276 amino acids long, $30 \mathrm{kDa}$ transmembrane protein with $4 \alpha$-helixes spanning the membrane (Greer \& Lees, 2002). It is encoded by a $15 \mathrm{~kb}$ long 7 -exons gene on the X-chromosome that could be also alternatively spliced to yield a minor PLP isoform, known as DM20. These two isoforms share a completely identical sequences, except of 35 amino acids segment encoded by $5 /$ part of exon 3 absent in DM20 isoform.

Isoelectric point of PLP lies within a strongly basic region, albeit PLP is highly hydrophobic. Presence of covalently-bound long-chain fatty acids, mostly palmitic acid, increases the hydrophobicity of PLP. PLP and DM20 isoforms are translated in the oligodendrocytes by the membrane bound polysomes and are transported through the Golgi to the plasma membrane. The turn-over of PLP occurs within 90 days (Greer \& Lees, 2002).

The oligodendrocytes of PLP-null mice have a condensed intraperiod line and decreased physical stability that confirms a likely role of PLP in compaction and stabilisation. Nevertheless, these mice can assemble compacted myelin sheaths. In addition, during development PLP mutants gain widespread axonal swellings and, with time, axonal degeneration, hinting on a role of PLP in axonal survival and maintenance (Klugmann et al., 1997; Jurevics et al., 2003; Rosenbluth et al., 2006).

PLP gene expression is reported to be associated with a general increase of oligodendrocyte number, suggesting a role of PLP in oligodendrocyte development. Moreover, naturally occurring point mutations or duplications in the PLP lead to the premature apoptosis of oligodendrocytes, and, as a result, reduced amount of white matter, ultrastructural changes of the myelin and general reduction of the myelin amount (Yamada et al., 1999). These mutations result in a severe dysmyelinating disease, for example Pelizaeus-Merzbacher disease (PMD) and spastic paraplegia type 2 (SPG2). 


\subsubsection{Myelin oligodendrocyte glycoprotein, MOG}

In contrast to the other protein in the nervous system, MOG is only a CNS featured protein. It is a quantitively minor component of the myelin sheath, representing only a $0.05-0.1 \%$ of the total myelin protein amount. It has a molecular weight of $26 / 28 \mathrm{kDa}$ (Abo et al., 1993) and is enriched in the outermost compact regions of the cultured oligodendrocytes and in the periphery of the compact myelin sheaths in the CNS (Figure 1.4) (Brunner et al., 1989).

MOG X-ray crystal structure elucidation, using a protein from a Norway rat, revealed it to be a 218 amino acids long transmembrane type I protein from the immunoglobulins (Ig) superfamily. It possess a single Ig variable domain ( $\mathrm{Ig}-\mathrm{V})$ on the N-terminus, exposed to the extracellular space (Berger \& Reindl, 2007; Iglesias et al., 2001). MOG glycoprotein moiety consists of $80 \%$ of N-linked oligosaccharides of the complex type. The C-terminus is pretty short and highly conserved between species (Iglesias et al., 2001). MOG possess additional hydrophobic stretch that does not span the membrane. It is either tightly associated with, or integrated into membrane from the cytoplasmic face (Pham-Dinh et al., 1995).

MOG was identified as a major target for autoantibody-mediated demyelination in experimental autoimmune encephalomyelitis (EAE) (Pham-Dinh et al., 1995). Therefore, it was a major suspect in the pathogenesis of multiple sclerosis (MS) (Iglesias et al., 2001).

The precise function of MOG in the CNS is still unknown, however its Ig-domain suggests it to be a receptor, or to play a role in the adhesion. Highly conserved C-terminus on the cytosolic part hints to the interactions of this protein with cytoskeleton (Dyer \& Matthieu, 1994). Cellular expression of MOG is usually delayed, comparing to the other myelin components, thus MOG is a perfect marker for the mature oligodendrocytes (Solly et al., 1996). Moreover, it suggests the role of MOG in completion, compaction and maintenance of myelin in adolescence (Johns \& Bernard, 1999).

\subsubsection{Myelin-associated glycoprotein, MAG}

MAG is one of the minor myelin compound representatives, contributing only $0.1-1 \%$ to the total myelin protein amount. It is restricted to the non-compact regions of the oligodendrocytes and in vivo is enriched exclusively in the periaxonal membrane of myelin internodes (Figure 1.4) (Sternberger et al., 1979). Structurally, MAG is a type I transmembrane protein and consists of $5 \mathrm{~N}$-terminal Ig domains on the extracellular space and one of two possible alternatively spliced cytoplasmic domains (Burger et al., 1992). Its overall molecular weight is $100 \mathrm{kDa}$, with $30 \%$ of it being heterogenous N-linked oligosaccharides (Frail \& Braun, 1984). Most of them are negatively charged due to sialic acid residues or sulphates (Quarles, 2007). Extracellular domain of MAG comprised 5 glycosylated Ig domains, with N-terminal being of variable type and 2-5 being of constant type. There are an interdomain disulphide bridges between domain 1 and 2 (Pedraza et al., 1990).

MAG is a product of an alternative splicing with 2 isoforms, differing only by their carboxyterminal segments. Expression pattern of these isoforms is strictly regulated during development, with a short version of MAG (S-MAG) being broadly expressed in all non-compacted myelin membrane, while L-MAG (long version) is restricted 
only to a periaxonal membrane (Quarles, 2007).

Over past decades, many interaction partners of MAG were reported: tubulin and protein kinase $\mathrm{C}$ for S-MAG; protein kinases $\mathrm{C}$ and A, Fyn kinase, phospholipase C $\gamma$, Src kinase for L-MAG (Kursula et al., 2000; Jaramillo et al., 1994; Afar et al., 1990; Kursula, 2001; Kursula et al., 1999). Moreover, the presence of Ig domains on the N-terminus of MAG, enables it to bind to sialic-acid containing oligosacharides on other glycoproteins and some gangliosides (Varki \& Angata, 2006). This feature of MAG indicates a presence of many possible interaction partners outside the oligodendrocyte, first of all on the axolemma (Quarles, 2007). MAG also is subjected to the post-translational modifications, phosphorylation of the cytoplasmic domain by protein kinase C being one of them (Quarles, 2007).

\subsection{Lipid behaviour and their dynamical properties within the membrane}

\subsubsection{Historical overview of the cell membrane architecture investigations}

The first insights in the field of membrane structure studies arrived from the physical and chemical studies of the interaction of oil on the surface of water: back into 1890, lord Raleigh conducted a series of experiments that resulted in calculation of thickness of the oil film on the water (Tanford, 1989). A year later, the unique device to study the exact area of an oil film was build in the kitchen of a German woman - Agnes Pockels. The principle of this machine is still used nowadays, however, it is now refereed as a Langmuir apparatus, since some improvement was done by Irving Langmuir (Langmuir, 1917).

About the same time it was discovered by Charles Ernest Overton that nonpolar substances may pass quickly through the plant membrane into the cell. Based on this it was proposed for the first time that cell membrane of a plant has some similarities with oil (olive oil) and that some molecules pass through the membrane by "dissolving" the lipid structure (Tanford, 1989). Later, with development of microscopy, biologists started to pay more precise attention to the cellular membranes.

In 1917 the publication by I. Langmuir arrived where he proposed that fatty acid molecules form a monolayer on the surface of water by orienting themselves in a specific way: hydrocarbon chains away from water, and carboxyl groups towards it (Langmuir, 1917). Only after decades one could realise the key importance of that publication.

The first classical experiment in the field of animal cell membranology has been conducted by Gorter and Grendel. Using lipids, extracted from red blood cells they could show that the lipid molecules could form a bilayer (Gorter \& Grendel, 1925). Even though it was not postulated as a model at that time, it actually may count as a first description of the animal cell membrane composition, even if several mistakes during the experimental set-up had occurred (Sadava, 1993).

The first membrane model appeared in 1935, when Danielli and Davidson further broadened the observation of Gorter and Grendel, postulating that the membrane is a 'sandwich' (bilayer) of lipids, covered on both sides with proteins. Later, they 
included protein lipid pores and areas of high protein activity (Danielli \& Davson, 1935).

Along the development of electron microscopy technique, the membrane model encountered some further development. The 'unit membrane' model was suggested by Robertson, based on electron microscopy studies. Under magnification of transmission electron microscope, membrane appeared as two outer dark lines (extracellular and intracellular protein layers) with an inner lighter line (lipid bilayer) (Robertson, 1960).

The fulcrum of the membrane field studies arrived in the early 1970s. The fluidmosaic membrane model was developed by Singer and Nicolson (Singer \& Nicolson, 1972). This model rendered the previous model inadequate and established a new paradigm. Proteins were regarded now as globular entities, floating within the lipid bilayer, rather than forming an outer and inner layers around it. Furthermore, the complete structure has been viewed as entirely fluid, with all the components undergoing free diffusion. The model also postulated that proteins are present within the membrane in rather low concentrations (Figure 1.5).

Membrane proteins were further studied by Unwin and Henderson, revealing that lipid-spanning portion of the proteins has hydrophobic nature and the one out of the bilayer is hydrophilic (Henderson \& Unwin, 1975).

The Singer and Nicolson membrane model became a 'golden standard' in the membranology. However, as important as this model is for the field, the rapid developing and improvement of the light microscopy techniques and, therefore, new findings over past 40 years had rather weakened its generalised concept. Proteins are not anymore seen as a scarcely distributed within the membrane, the bilayer itself is known not to be a smooth sea of homogeneously distributed lipids and the free diffusion is not considered as the prevalent type of the motion of the membrane components.

\subsubsection{Types of lipid dynamics}

Breaking the diffraction limit of light microscopy, STED, PALM/STORM and single particle tracking (SPT) with quantum dots, greatly advanced the studies of membrane architecture and organisation. Besides the static disposition of membrane molecules, also the dynamical perspective of the ensemble could be assessed: trajectories of lipids and proteins throughout the membrane could be visualised. Studies on molecular dynamics became very important tools in the investigation of how the plasma membrane is built and how it functions.

\section{Free diffusion}

Brownian motion on a macroscopic scales is called free diffusion: due to thermal agitation processes, molecules are constantly on the move, colliding with each other and thus bouncing back and forth. General features of free diffusion are:

- the higher the temperature is, the faster diffusion occurs,

- the higher the molecular density is, the higher the possibility of molecular collision that slows down the diffusion, 
A

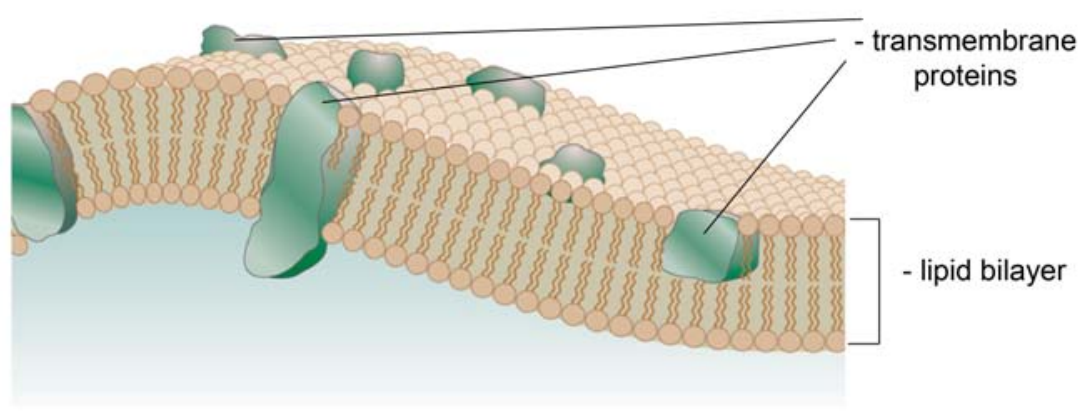

B

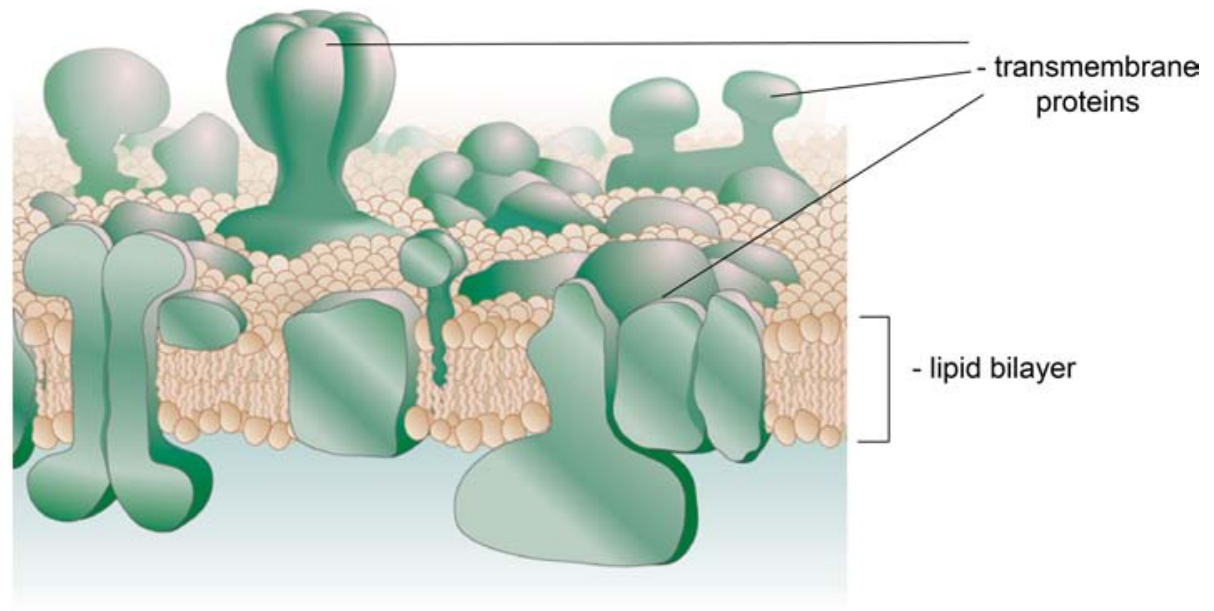

Figure 1.5: Schematic overview of the membrane models. (A) The original Singer-Nicolson "fluid mosaic model": lipid sea with globular protein islands in it. (B) An updated version, with proteins being not only globular, but properly transmembrane and integrated through the whole length of the membrane. From (Engelman, 2005).

- due to the random nature of the forces generated by molecular collisions, there is no directionality of molecular movements, therefore diffusion creates a homogenous distribution of molecules.

As mentioned above, the lateral homogeneity of the membrane was an important hallmark of the fluid-mosaic membrane model of Singer and Nicolson. In this diffusion model, the diffusion coefficient, D, depends only on the hydrodynamic radius of the protein (was assumed to be cylindrical) and the viscosity of the membrane. Therefore, distance a molecule diffuses, measured as a mean square displacement in time $t$, relates linearly to the diffusion coefficient $\mathrm{D}$ :

$$
M S D=4 D t^{\alpha}, \alpha=1
$$

(Free diffusion)

Free diffusion might become a directional flow, when the transport of the molecule is assisted by other compounds. Diffusion coefficient of the flow is slower than the free diffusion, and equation takes into account the flow velocity value squared, $v$ (Owen et al., 2009).

$$
M S D=4 D t+v^{2} t^{2}
$$

(Flow directed diffusion) 


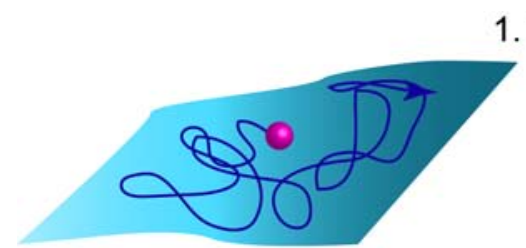

A Brownian motion
1. Free diffusion

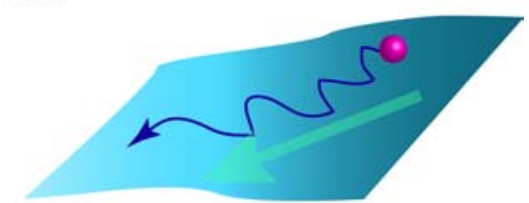

B Diffusion with flow

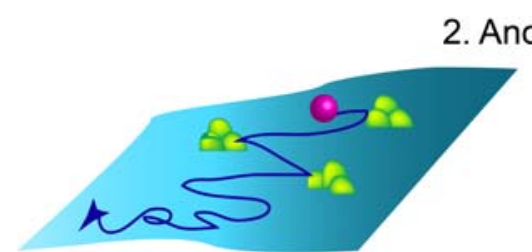

C Impermeable obstacles

2. Anomalous diffusion

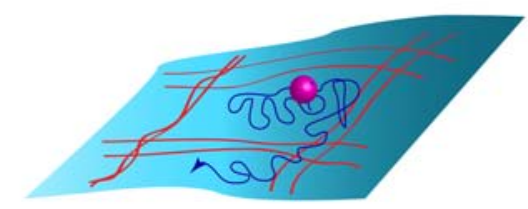

E Cytoskeleton meshwork: hopping

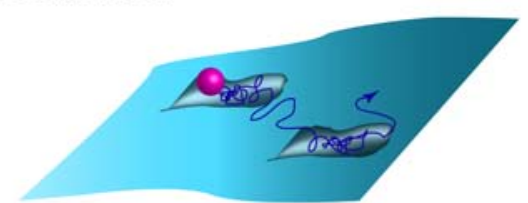

D Lipid microdomains: trapping

Figure 1.6: Schematic overview of the models, characterising the events of dynamics within the membrane. (A) Brownian motion. (B) Flow directed diffusion. (C) Impermeable obstacles. (D) Lipid microdomains. (E) Cytoskeleton meshwork, together with proteins that are aligned along it. See text for explanations. Modified from (Owen et al., 2009).

\section{Anomalous diffusion}

Upon development of artificial membrane models (solid supported bilayers, large and giant unilammelar vesicles, liposomes) it was observed that diffusion in the plasma membrane for many different lipid probes and proteins (GPI-anchored mostly) was slower than the one measured in artificial lipid membranes. Various models were proposed to explain this phenomenon, and the diffusion coefficient retardation was explained by the following causes (Figure 1.6):

- protein-protein interaction, oligomerisation and large multimeric complexes formation (Douglass \& Vale, 2005; Marguet et al., 1999);

- active transport, cytoskeleton interactions with lipids and proteins, confinement of the proteins cytoplasmic tails within actin meshwork (results in direct and indirect immobilisation) (Kusumi \& Suzuki, 2005; Eggeling et al., 2009; Sahl et al., 2010; Mueller et al., 2011);

- membrane lipid microdomains formation (Simons \& Ikonen, 1997; Simons \& Vaz, 2004; van Meer, 2005).

Based on this, the $\alpha$ coefficient in the free diffusion equation deviates from 1 , and diffusion becomes anomalous, loosing its linear relation to time.

$$
M S D=4 D t^{\alpha}, \alpha<1
$$

(Anomalous diffusion)

Diffusion rates within heterogenous cell membranes depend on a scale. Even though the global values for diffusion over macroscopic regions of the membrane 
might indicate a free diffusion, on the nanoscale an anomalous diffusion might occur (Kusumi \& Suzuki, 2005). Nanoscopy approaches (single-molecular tracking, STED, FCS-STED) are of a great importance in the study of this processes (Eggeling et al., 2009; Sahl et al., 2010; Mueller et al., 2011).

Depending on the nature of the lateral diffusion constraint, several types of anomalous diffusion are described.

Hop diffusion within cytoskeleton meshwork. In 1980 Sheetz et al. demonstrated that lateral diffusion was faster in the presence of a disorganised cytoskeleton meshwork (Sheetz et al., 1980). Usage of single molecular tracking techniques by Kusumi and co-workers proposed how this dependence may occur and fence and pickets model was suggested in 2005. According to it, the cytoskeleton meshwork forms fences and transmembrane proteins, anchored with their cytoplasmic tails to this meshwork form pickets (Kusumi \& Suzuki, 2005). Comparing to the fences in the farmers field, the proteins, aligned along the cytoskeleton meshwork form a compartment (200 nm scale) and restrict the free passage of the other molecules between two adjacent compartments (Figure $1.7 \mathrm{~A}$ ). Molecules, locked within a compartment between fences undergo fast free diffusion. Upon either thermal fluctuation, or fence protein oligomerisation, the boundary opens and the molecule slowly diffuses to the other compartment. Such diffusion mode alteration - fast within a compartment and slow between adjacent compartments - was termed hop diffusion or hopping (Kusumi \& Suzuki, 2005). Pickets formed by transmembrane proteins trespass the whole thickness of the membrane and influence molecular diffusion of the lipids and GPI-anchored proteins in the outer leaflet (Fujiwara et al., 2002).

Trapping within lipid microdomains: membrane partitioning. Microdomains are regions of the membrane with changed characteristics. The most deviating factors are membrane order, fluidity and protein concentration. The first two features are highly dependent on cholesterol and sphingolipids levels, since enrichment in these particular lipids increases order and decreases the fluidity. Often such cholesterol-enriched microdomains contain higher concentration of proteins, building up functional platforms (Figure 1.7 B) (Brown \& London, 2000; Simons \& Vaz, 2004). Increasing evidence about microdomains existence crush the initial idea of the membrane homogeneity, since alteration in the membrane order and fluidity have a severe impact on the diffusion. It was shown that microdomains resident proteins exhibit a lower diffusion rate than proteins outside the domains (Shvartsman et al., 2003).

Lower diffusion rate within these domains is often referred as a trapping: diffusing molecules reside within the domain for the time $t$, either due to the direct interactions with one of the domain constituents, or just due to the physical properties (order/fluidity) that hinder the fast diffusion. The nanoscale size of lipid domains is a challenge for their excessive study. However methods, such as FCS (fluorescent correlation microscopy), nowadays often combined with STED facilitate these studies (Eggeling et al., 2009; Sahl et al., 2010).

An original FCS approach, developed by Wawrieziniec in 2005 - FCS diffusion law - provides not only an information about diffusion coefficient, but also sheds light on the type of membrane organisation (Wawrezinieck et al., 2005). This approach 
Intracellular side

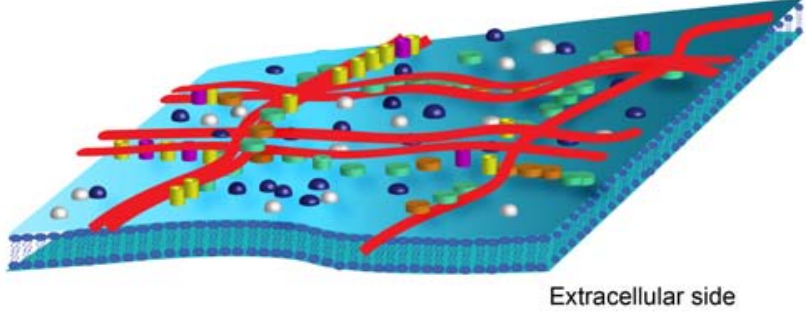

B Membrane partititioning model

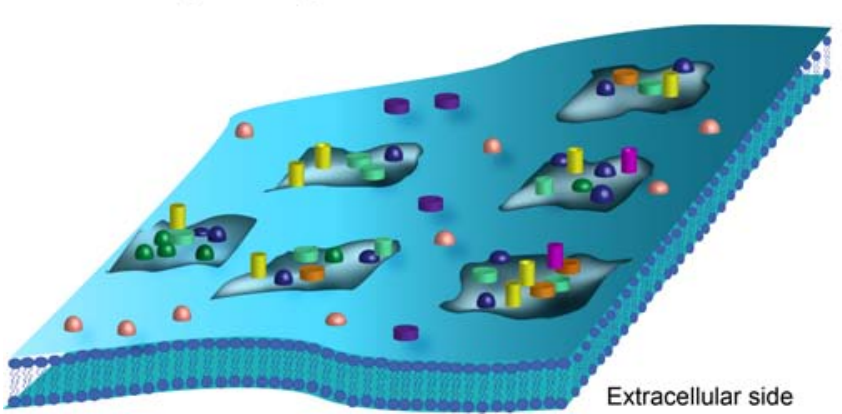

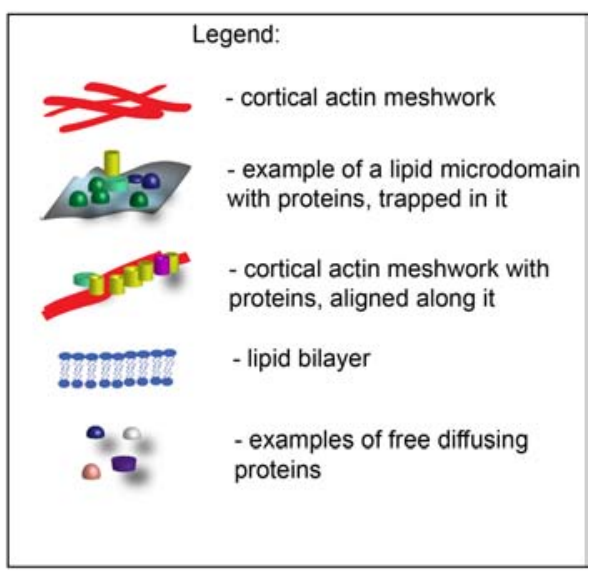

Figure 1.7: Schematic overview of the cytoskeleton "fence and pickets" trapping models, and membrane partitioning model. (A) Cytoskeleton meshwork, together with proteins that are aligned along it, compartmentalise the membrane into nanodomains, perturbing free random movements. Molecule within this compartment still undergo fast free diffusion, bouncing from the "fences" of compartment. Upon thermal fluctuations or some structural changes of the cytoskeleton, the hallway opens and molecule can slowly squeeze to another compartment, where it resembles free diffusion fast mode. This changes of the dynamics from fast to slow and back to fast movements is named hopping. (B) Lipid microdomains: diffusing molecule is interacting with the constituents of the microdomains (signalling cascade, receptor binding, fusion, etc), therefore remains trapped within the domain for some time. Free movement is resolved upon the interaction abort. Modified from (Owen et al., 2009).

was successfully used to distinguish diffusion mode within lipid microdomains from one dependent on the actin meshwork (Lenne et al., 2006). Further combination of FCS setup with STED empowers this method, accessing diffusion behaviour on the nanoscale levels. For instance, Eggeling and coworkers used the STED-FCS method to provide a comprehensive study of the anomalous diffusion behaviour of lipids and impact of the actin meshwork and cholesterol concentration on the lipid diffusion (Eggeling et al., 2009; Mueller et al., 2011).

Impermeable obstacles: membrane environment and protein complexes formation. Proteins of the inner cellular membrane (ER, Golgi) were shown to be more mobile, suggesting that their lateral diffusion in these organelles is less constrained (Cole et al., 1996). However, a decrease in the values of diffusion coefficient was shown for the ER proteins, involved in the functional large complexes formation (MHC class I and TAP proteins) (Marguet et al., 1999).

It was suggested by Gambin that the diffusion value may be strongly affected by protein dimensions (Gambin et al., 2006). Therefore, protein and lipid diffusion might be altered not only by physical barriers within the membrane, but also by molecular complexes formation and protein oligomerisation. Molecules either slow down due to direct interactions, or due to indirect bouncing off the obstacle on their way (Marguet et al., 2006). 


\subsubsection{Myelin as a unique membrane in a context of the lipid diffusion}

Dynamical characteristics of the major constituent of the myelin membrane lipids - haven't been studied, due to the structure complexity of the myelin sheath and absence of a reliable measurement setup. It was shown, however, that myelin is a very robust system, since radioactively marked lipids remained within myelin within years (O'Brien, 1965b). It remained unknown, however, whether this robustness is combined with membrane rigidity, leading to a diffusion blockade and subsequently low turn-over.

As has been described in previous sections, myelin is enriched in cholesterol, sphingomyelin. Moreover, myelin lipids often possess high number of long-chain fatty acids in their structure. These facts might suggest a rather ordered membrane composition and, therefore, a slow diffusion rate.

However, myelin is scarce in proteins, and due to the MBP properties, cytoplasm and any cytoplasmic components are squeezed out from the compacted regions. Actin meshwork also falls under this rule and it has been experimentally proven that compacted myelin lacks filamentous actin (Aggarwal et al., 2011). Low protein crowding and no actin meshwork turn the scale to favour of unrestricted diffusion within myelin sheath.

Taking all facts about myelin membrane features into account, what would be the answer to the question: what is the diffusion behaviour of the lipids in compact myelin? The Part I of my thesis Results Chapter is dedicated to finding a solution to this puzzle. 


\subsection{Cell polarity}

Under the common term cellular polarity one describes the asymmetric organisation of the physical aspects of the cell, including cellular surface components, intracellular organelles distribution and cytoskeleton rearrangements. Polarity is a ubiquitous feature among all species in all classes of eucariotes, from the unicellular yeast, to the multicellular organisms. Cellular polarity of the plasma membrane is a potent and important regulator involved in all crucial organismal life points, from the global events of cellular migration, tissue formation and differentiation in the development, to the more minor, such as epithelium function maintenance, blood cell migration, cellular division, etc.

Evolutionary, epithelial cells are the most typically polarised cells and approximately $60 \%$ of mammalian organism is of epithelial or epithelia-derived origin. Therefore, the most studies on the polarity were performed on the simple columnar epithelium of the mammalian intestine or kidney. Membrane of the epithelial cells is polarised into two distinct domains: apical and basolateral. Apical surfaces faces inward to the lumen and are specialised mainly for the material exchange. Basolateral surface, in contrary, promote contacts with the adjacent cells and extracellular matrix (ECM) to provide stability. Therefore, depending on the functional differences, both cellular parts developed under the polarity control to be strictly organised to fulfil special needs.

This functional specifications of the cellular membrane, determining cell orientation, function and fate, occur almost in all cell types. To name a few: neural synapses are specialised into transmitter release and uptake sites; photoreceptors are divided into regions of light-sensing activity and connection to the neurons; migrating cells exhibit asymmetric front-back polarity upon movements towards attractive cues.

One may suggest that to acquire all these different functions a diverse mechanisms for the polarisation control should be involved. Strikingly, that is not the case. There are several common mechanistic themes, simple variation of which results in the perfect orchestration of the polarity events.

Common mechanisms of cellular polarity include:

- Recognition of the intrinsic protein code that determines the part of the cellular membrane the protein will be delivered to by cytoplasmic adaptor complexes.

- Specific association of the signalling complexes and scaffolding proteins from the cytosolic side with the appropriate part of the membrane. They define and stabilise the biochemical features of the building domains.

- Adhesion receptors orientate cells in the three-dimensional space by sensing the neighbouring cells and extracellular matrix.

The idea of these mechanisms to be conserved among all cells rises mainly from two considerations: all cell share common cellular machineries for protein trafficking; all cell types are able to lose polarity (cancer circumstances) trough epithelialmesenchymal transition, disintegrate from its original tissue, migrate and undergo reorganisation upon reintegration at the new site (Wodarz \& Näthke, 2007; Thiery \& Sleeman, 2006). 


\subsubsection{Overview of the mechanisms of the cellular polarisation} Sorting, trafficking and retention control

'Boarding pass' to the right vehicle. Protein sorting and post-translational modification occurs step-wise within endoplasmatic reticulum (ER) and Golgi system. Three classes of proteins were identified so far to be responsible for the cargo packing into vesicles for the delivery:

- coatomer protein complex-II (COPII) for trafficking from ER to Golgi;

- coatomer protein complex-I (COPI) for delivery from Golgi to ER, and within Golgi network itself;

- the adaptor protein (AP) clathrin complex is crucial for transport between the Golgi, the plasma membrane and endosomes.

All these systems use as a 'boarding pass' the specific sorting signal on their protein cargo. Regardless the destination, vesicle transport is mediated by a 'railway roads' of the cells, namely: actin filaments and microtubules. Further fusion of the delivered vesicles with the point of destination is regulated by organellespecific small GTPases (Rabs), vesicle-tethering complexes and SNAREs (Hehnly \& Stamnes, 2007; Grosshans et al., 2006). Upon cellular polarisation these ubiquitous mechanisms are modified and used to sort proteins into separate plasma membrane domains.

Cytoskeleton involvement into sorting. To further ensure the precise localisation of the sorted cargo, polarised cells possess a specialised organisations of actin filaments and microtubules. Actin filament underlie the entire plasma membrane and interact with membrane proteins via ankyrin-spectrin complexes (Bennett \& Healy, 2008). Since actin filaments are closely regulated by small GTPases of the Rhofamily that are involved in several crucial polarisation points, they might indirectly have an impact on the protein sorting (Rodriguez-Boulan et al., 2005; Schmidt \& Hall, 1998).

Fusion of the sorted proteins with the targeted membrane. Subsequent fusion of the vesicle with the correct membrane is a next crucial step in the polarity generation. Special exocyst complex (involves several Rab GTPases) regulates the docking of a subset of basolateral vesicles, whereas annexin complexes are involved in the fusion with apical membrane of epithelial cells (Grindstaff et al., 1998; Gerke et al., 2005; Ang et al., 2004). SNARE complexes (v)-SNAREs (vesicle-associated membrane protein -VAMP) and target (t)-SNAREs (the syntaxins) regulate the final step of the vesicle and plasma membrane fusion (Rothman, 1994).

Protein retention within the right domain. The next step after precise recruitment of the membrane proteins into specialised domains is their retention within these membrane patterns.

Scaffolding proteins complexes, such as ankyrin-spectrin, may interact with the cytoplasmic domains of the membrane proteins, anchoring them in place. Several 
classes of membrane proteins are sequestered in this way, including ion transporters and channels (such as the anion exchanger, $\mathrm{Na}^{+} / \mathrm{K}^{+}$-ATPase, voltage-gated $\mathrm{Na}^{+}$ channel and $\mathrm{Na}^{+} / \mathrm{Ca}^{2+}$-exchanger), receptors (such as $\mathrm{Ins}(1,4,5) \mathrm{P} 3$ receptor, ryanodine receptor and $N$-methyl- $D$-aspartate receptor (NMDA-R), and cell-adhesion proteins (such as E-cadherin, L1 (Ng-CAM) and CD44) (Mellman \& Nelson, 2008).

Diffusion barrier is another option to separate membrane domains that are otherwise part of a continuous lipid bilayer and prevent intermixing of the polarised regions. This barrier in epithelial cells comprises the tight junction and is placed directly to the boundary between apical and basolateral domains. Tight junctions create a natural fence within membrane, preventing free diffusion from one domain to the other (Shin et al., 2006). Only a regulated vesicular transport, such as transcytosis, can overcome this barrier. In the absence of the tight junctions, diffusion barrier might be created in a different way. Targeted localisation of the scaffolding proteins and cytoskeleton tend to crowd membrane proteins, increasing their concentration within a particular region of the plasma membrane, therefore impeding the free diffusion of the molecules. Membrane of the axon initial segment, for example, has such kind of barrier to restrict axonal membrane proteins to diffuse into soma (Winckler et al., 1999). Immunological synapse, and budding yeasts are another examples (Mellman \& Nelson, 2008).

\section{Intrinsic cues of the cellular polarity: how does the cell know where to sort?}

The sorting mechanism in the polarised cell is a complicated machinery that controls where the newly synthesised proteins will be departed. However, what are the cues for the cell to orientate within a multicellular context and distinguish between its own 'floor' and the 'ceiling'?

Protein complexes: PAR, Scribble and Crumbs. PAR (partitioning defective) complex consists of 6 PAR proteins (PAR-1 to 6) and is required to establish polarity in early C. elegans development (Kemphues et al., 1988).

It is suggested that Ser/Thr kinases PAR1 and PAR4 are involved in the regulation of at least two key points of the polarisation event: post-Golgi vesicle delivery and their docking and fusion at the plasma membrane (Mellman \& Nelson, 2008). Apart from the kinases, PAR complex comprise PAR3 (Bazooka or Baz) and PAR6 proteins with multiple PDZ domains, 14-3-3 homology protein PAR5 and RING-finger protein PAR2 that function as a scaffold of the PAR complex (Suzuki \& Ohno, 2006; Goldstein \& Macara, 2007). PAR-3 is the first protein of the PAR/aPKC complex to show strictly apical localisation during epithelial polarity establishment (Harris \& Peifer, 2004). PAR3 and PAR6 actively recruit Cdc42 and atypical protein kinase $\mathrm{C}$ (aPKC). They form a subcomplex that localise to apical junctional complex in polarised epithelial cells or to the tips of axons in neurons (Mellman \& Nelson, 2008). PAR3-PAR6-aPKC subcomplex, positioned at the crucial apical-basolateral junction, contributes further to the establishment and control of maintenance of apico-basal polarity in embryonic epithelial cells and to axon formation in neurons (Mellman \& Nelson, 2008).

Basolateral identity is regulated by Crumbs complex that is placed below the 
apical junctional complex and along the membrane at cell-cell contacts. The apical part of the membrane is under control of Scribble complex, localised to the apical side of the junctional complex in polarised epithelial cells. Loss of function studies of either of these complexes revealed drastic defects in the polarity establishment due to the reduction of the surface area of the apical and basolateral plasma membrane domains. However, as in case of PAR1 and PAR6, the precise mechanism of the polarity regulation by Crumbs and Scribble remains unknown (Figure 1.8) (Tanentzapf \& Tepass, 2003; Bilder et al., 2003).

Role of phospholipids. Apart from the protein polarity complexes, cell use phosphoinositols to establish and maintain their polarity. Among plethora of phosphoinositol species residing in a cell, $\mathrm{PIP}_{2}$ and $\mathrm{PIP}_{3}$ exhibit the key impact during cellular polarisation events. Local changes in these phosphoinositides moieties within cellular membrane is acquired via distinct localisation of the two enzymes: phosphoinositide 3-kinase (PI3K) and phosphatase and tensin homologue (PTEN) phosphatase. PI3K generates phosphatidylinositol-3,4,5-triphosphate $\left(\mathrm{PIP}_{3}\right)$ and PTEN removes the 3/phosphate from $\mathrm{PIP}_{3}$ to yield phosphatidylinositol-4,5-bisphosphate $\left(\mathrm{PIP}_{2}\right)$ (Figure 1.8) (Sasaki et al., 2009).

Spatial and functional separation of these lipids is very abundant. The tip of the growing axon is enriched in $\mathrm{PIP}_{3}$ (Shi et al., 2003), as is the leading edge in migrating cells. In contrary, $\mathrm{PIP}_{2}$ is strictly sequestered to the trailing region of the migrating cells. In the epithelia, $\mathrm{PIP}_{3}$ is usually restricted to the basolateral part, while $\mathrm{PIP}_{2}$ is found in the apical part. The enzymes follow the respective pattern: PI3K is localised to the basolateral domain and the tip of the growing axon and PTEN resides in the apical part of the epithelial cells and in the trailing region of the migratory ones (Shewan et al., 2011).

Upon inhibition of PI3K activity, neurons lack axonal differentiation (Shi et al., 2003) and apico-basal axis in epithelial cells becomes misoriented (Gassama-Diagne et al., 2006).

Based on the severals studies, PTEN phosphatase was suggested to be a key regulator of the apical membrane interface establishment. It was shown that PAR3 protein of the PAR3-PAR6-aPKC complex, which has apical asymmetry, binds via its PDZ domain to the PTEN and this binding is necessary for the epithelial cell polarity establishment (von Stein et al., 2005; Feng et al., 2008). Moreover, PAR3 can also directly bind to $\mathrm{PIP}_{3}$ and $\mathrm{PIP}_{2}$ (Krahn et al., 2010). These findings introduce a possible coordinatory role of the PAR3 during apical interface specification: on the one hand PAR3 may recruit PTEN to the apical surface that leads to a subsequent removal of $\mathrm{PIP}_{3}$, thus removing the basolateral identification. Simultaneously, the production of $\mathrm{PIP}_{2}$ occurs, resulting in the establishment of apical membrane identity.

Of course, several other factors to localise and stabilise PAR3 on the apical surface are needed in this scenario, otherwise PAR3 would randomly bind to PTEN and $\mathrm{PIP}_{3}$ all over the cell. Moreover, PTEN alone is not sufficient to produce all $\mathrm{PIP}_{2}$ needed. It is most likely responsible only for the swift elimination of $\mathrm{PIP}_{3}$. Phosphatidylinositol 5-kinase, (PI(5)-kinase), an enzyme, known to stimulate $\mathrm{PIP}_{2}$ production, was found to be localised to the apical surface of MDCK cells, therefore being a candidate, responsible for the accumulation of $\mathrm{PIP}_{2}$ in this region (Guerriero et al., 2006). However, additional mechanisms of $\mathrm{PIP}_{2}$ concentration are 
still under consideration.

There is still lack of understanding how the asymmetry of phosphatidylinositols occurs at the first place. The general existing model favours the involvement of signals, generated via interactions with the surrounding extracellular matrix (ECM) (O'Brien et al., 2002). In the MDCK cells $\beta$-integrins are the crucial components in the cell-ECM interactions and are known to modulate PI3K activity and $\mathrm{PIP}_{3}$ levels (Parise et al., 2000; Yu et al., 2005). Therefore, one may suggest that specification of the basolateral pattern begins with enrichment of the $\mathrm{PIP}_{3}$ and PI3K on the plasma membrane, upon control of the $\beta$-integrins. Cell-cell contacts formation via E-cadherins and their impact on the PI3K activation might be a next step in the membrane specification (Kovacs et al., 2002). Elevated $\mathrm{PIP}_{3}$ levels trigger the avalanche recruitment of the effector proteins to further establish the axis of polarity and design the proper basolateral interface of the membrane.

As has been mentioned in the previous section, proper cytoskeleton organisation to provide proper shaping (migratory cells), stability support (epithelia) and vesicle transport of neatly sorted proteins should follow immediately after apical and basolateral surfaces are determined. Both phosphatidylinositols are known to be cytoskeleton modulators, however they orchestrate with slightly different outcomes (see overview in Section 1.3.2).

\section{Extrinsic cues of the cellular polarity}

Apart from the intrinsic cues, a cell needs to further sense its positioning towards other cells and its 3D orientation according to the extracellular matrix (ECM). The hints for it were acquired upon observing individual epithelial cells grown in suspension. In the absence of cell-cell and cell-ECM adhesion these cells do not develop polarity, but instead undergo programmed cell death. In contrary, when grown on the substrate, individual epithelial cells establish and maintain their apico-basal axis. Moreover, if this substrate corresponds to one of those that cells encounter in vivo, cells start to develop the appropriate functionality: neurons specifically form an axon, while single mammary epithelial cells selectively secrete $\beta$-casein from the apical surface and 3D epithelial cysts polarise correctly (Mellman \& Nelson, 2008). Thus, the presence of the ECM, and cellular adhesion to it, is of a great importance and provides the final orientation cues for the cellular polarity establishment.

The main polarity regulators are summarised on Figure 1.8.

\subsubsection{Polarity within the myelin sheath}

Myelin is highly differentiated and specialised structure that is functionally and structurally polarised into different domains (Figure 1.9).

Along the radial dimension of myelin, there is a division into non-compacted outer abaxonal and inner adaxonal membrane and compacted multilayered stacks. Along its longitudinal length myelin is subdivided into internodes, paranodes and juxtaparanodes (Baumann \& Pham-Dinh, 2001). Axonal plasma membrane upon contact with different areas of myelin also becomes polarised (Özçelik et al., 2010).

Oligodendrocytes in CNS, as well as Schwann cells in PNS are typically polarised cells. They exhibit segregation between plasma membrane of the cell body and myelinated region, with further division of the myelin into compact and non-compact 


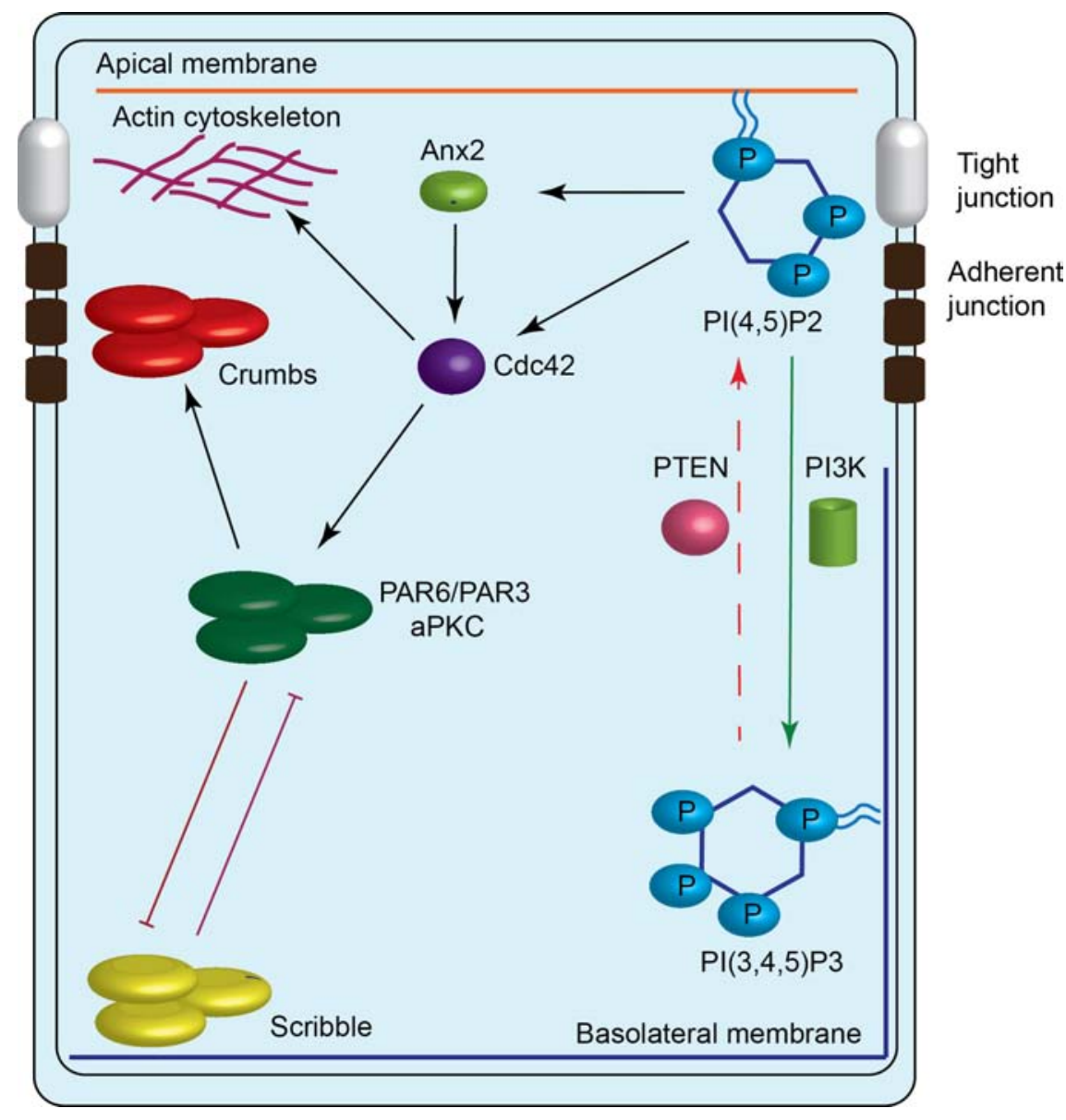

Figure 1.8: Schematic overview of the major complexes involved in the cell polarity establishment. Three main protein complexes are shown: Crumbs (red), PAR (dark green) and Scribble (yellow). Apical membrane is to the top, and basolateral to the bottom and the sides. All three complexes have an impact on each other localisation. PAR complex reinforces the localisation and activity (strictly apical) of the Crumbs, as shown with the arrowed black line. PAR and Scribble mutually antagonise each other (shown with the red lines), therefore controlling the precise location of each other: Scribble - strictly basolateral, PAR complex - to the apical junctional complex. In addition to the protein polarity complexes, lipid family of phosphoinositides are directly involved in the regulation of cell polarity. Two main players are $\mathrm{PIP}_{2}$ and $\mathrm{PIP}_{3}$, localised strictly to the apical and basolateral membrane, respectively. Together with phosphatidylinositols, enzymes responsible for their interconvertion are also targeted to different membrane domains: PI3K to the basolateral part and PTEN to the apical. These components regulate further either via Annexin2 or via Cdc42 cytoskeleton rearrangements that are important for the polar protein and vesicular trafficking.

domains. Compacted areas are enriched in lipids and harbour only few representative proteins (MBP and PLP in CNS), whereas composition of the non-compact region is similar to the cell body membrane, with CNP and MAG being predominant markers (Baumann \& Pham-Dinh, 2001).

Oligodendrocytes are remarkably independent from the axonal extrinsic cues in the establishing of their polarity, since upon cultivation in the absence of axons, oligodendrocytes form flat sheets that recapitulate compact myelin sheath in vivo with maintenance of all the aforementioned structures and with proper protein/lipid separation (Kachar et al., 1986). However, for the further completion of the compaction and wrapping, extrinsic cues from axons are required (Baumann \& Pham-Dinh, 2001).

The conserved principles involved in the membrane polarisation has been dis- 


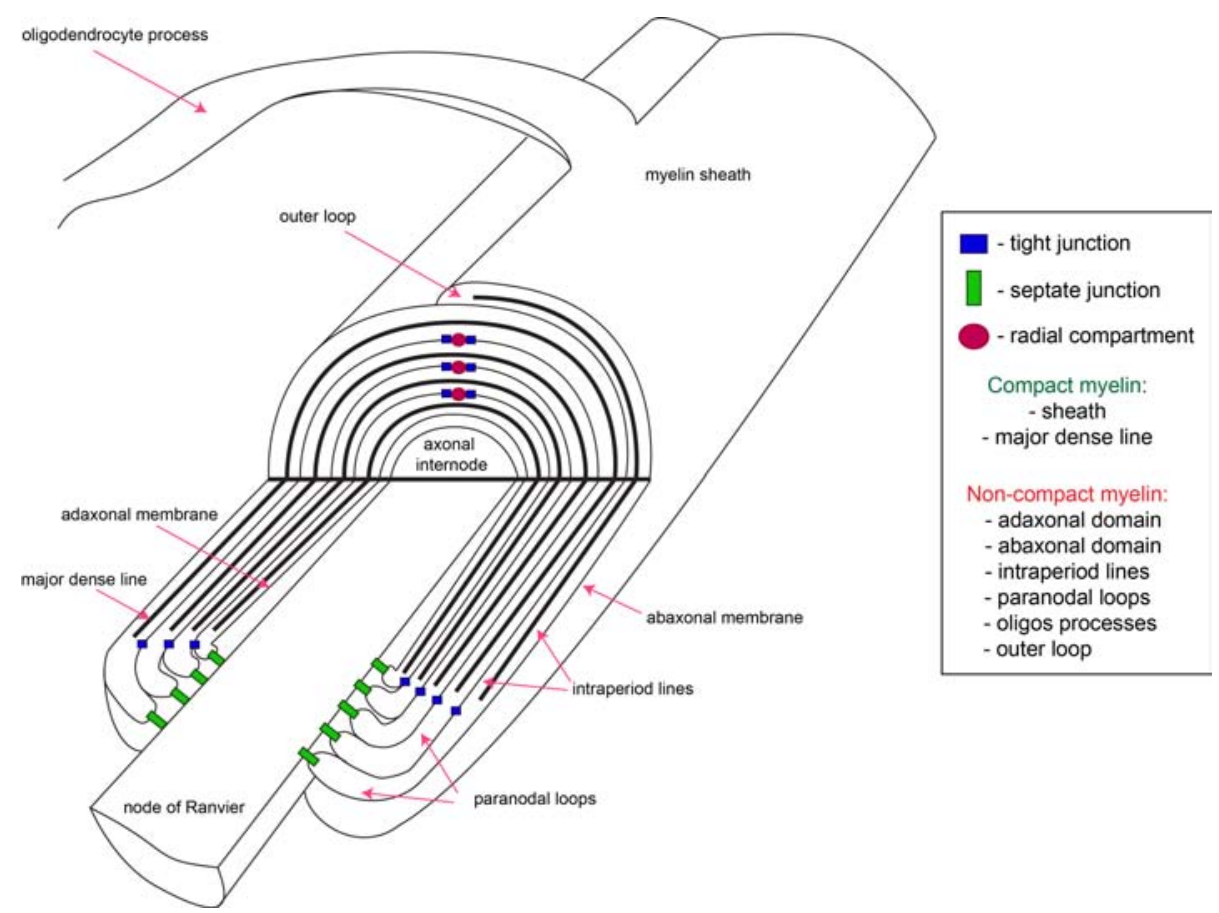

Figure 1.9: Schematic representation of the polarised domains in the myelin sheath in vivo. Compact and non-compact myelin domains are separated by tight junctions. Septate-like junctions connect paranodal loops to the axon (adapted from Maier, 2008).

cussed briefly earlier in this section. Along with using the ubiquitous mechanisms, oligodendrocytes are forced to develop some additional sophisticated ways for protein sorting and trafficking to overcome their own structural feature: tight packing of the myelin membrane (Simons et al., 2012).

It was shown that oligodendrocyte sheets lack the trafficking and sorting machinery, i.e., Golgi apparatus, ribosomes and vesicles (Aggarwal et al., 2011). Therefore, vesicle-mediated transport is confined to the cell body and non-compact processes. It is suggested that myelin membrane either preassembles within the biosynthetic pathway into tightly packed complexes, or is accumulated into endosomes that are subsequently delivered and incorporated to the myelin membrane upon extrinsic signal for the myelination (Simons et al., 2000). After incorporation into the membrane, myelin components are then delivered to the sheets region by membrane flow (Simons et al., 2012).

PLP is a good example of the involvement of the endosomal trafficking: it is found to be accumulated within the endosomes in the early stages of myelination and disappear upon myelination termination (Simons et al., 2000). On the other hand, MBP is translated on the borders of processes/sheets regions. Its mRNA is transported there via hnRNP granules trafficking (Barbarese et al., 2000). Upon translation, MBP interacts immediately with negatively charged lipids of the plasma membrane and is incorporated between the sheets. Its high adhesive properties might be the case why MBP is translated strictly at the places were two membranes are ought to be brought together, not to intervene with overall cellular architecture elsewhere in the cell. The precise mechanisms of the sorting and incorporation of the membrane components into oligodendrocyte sheets need to be further determined.

The role of the polarity complexes has been shown so far only for the Schwann cells in PNS. The outer membrane of Schwann cells (abaxonal domain) resembles 
a baso-lateral region of the epithelial cells, and inner membrane (adaxonal part) the apical region, with PAR and Scribble complexes defining the respective domains (Cotter et al., 2010; Özçelik et al., 2010). There are no evidence so far, though, that the same separation occurs in the oligodendrocytes.

Following sorting, membrane components need to be preserved within the destined domains. Diffusion barriers, build either by tight junctions or molecular fences (Figure 1.7) are usually responsible for this in the polarised cells. Primary oligodendrocytes do not form proper tight junctions between cell body and myelin-like sheet. However, tight junctions are present around the radial component of myelin, as well as between the internode and the paranodal loops, separating compact region from non-compact (Pedraza et al., 2001; Salzer \& James, 2003). OSP/claudin-11 is so far the only protein of tight junctions, found in oligodendrocytes (Bronstein, 2000). OSP/claudin KO mice, lacking tight junctions, result in a slower nerve impulse conduction and limb weakness, demonstrating the importance of these structures in the proper functionality of myelin (Gow et al., 1999).

Moreover, recent studies have shed the light on how MBP is involved into barrier formation between compact and non-compact zones. It was demonstrated that upon its self-assembly, MBP forms a sieve that prevents proteins with large cytosolic domains from entering the sheets (Aggarwal et al., 2011). The same study also claims MBP to be an active extruder that actively excludes proteins from the compact regions. Lipid-rich and protein-poor membrane is generated in this elegant way (Simons et al., 2012).

\subsection{Aim of the work}

Despite long years of being under investigation, myelin still remains enigmatic. In my thesis I aimed to investigate lipid dynamics within the oligodendrocyte sheets, as well as a distribution of $\mathrm{PIP}_{2}$ and $\mathrm{PIP}_{3}$ in there. Due to its special membrane characteristics, lipid behaviour within myelin sheath might drastically differ from the one, described so far for other cellular membranes. It would be of a great interest to investigate this, since lipid diffusion and type of motion would contribute to the knowledge of myelin membrane architecture and organisation. Continuing with the question of lipid organisation within the membrane, I investigated a distribution of phosphatidylinositols $\left(\mathrm{PIP}_{2}\right.$ and $\left.\mathrm{PIP}_{3}\right)$ within oligodendrocyte membrane at the different developmental stages. 
Chapter 2

\section{MATERIALS \& METHODS}

Theory guides. Experiment decides.

common knowledge 



\section{Materials}

\subsection{Chemicals and Consumables}

1. All chemicals, unless stated otherwise, were obtained from:

- Sigma-Aldrich (Sigma-Aldrich Chemie GmbH, Munich, Germany),

- Merck (Merck KGaA, Darmstadt, Germany),

- AppliChem (AppliChem GmbH, Darmstadt, Germany),

- PAA laboratories GmbH, Pasching, Austria.

2. All basal media, supplements, antibiotics and sera for cell culture, unless else is specified, were purchased from:

- Gibco/Invitrogen (Invitrogen GmbH, Darmstadt, Germany).

3. Consumables were purchased from:

- Falcon (Becton Dickinson Labware Europe, Le Pont De Claix, France),

- Eppendorf (Eppendorf AG, Hamburg, Germany).

4. CELLSTAR culture vessels were obtained from:

- Greiner Bio-One (Greiner Bio-One GmbH, Frickenhausen, Germany).

\section{$2.2 \quad$ Antibodies}

Primary antibodies used in this study are listed in Table 2.1. Antibody dilutions used for immunocytochemistry (IF) and western blot analysis (WB) are given in brackets. Secondary fluorophore-conjugated antibodies were purchased from Dianova (Hamburg, Germany) and used in 1:200 dilution for IF and 1:2000 dilution for western blot analysis. 
Table 2.1: Antibodies

\begin{tabular}{|c|c|c|c|}
\hline Target & Host species & Application & Reference \\
\hline AA3 (PLP) & Mouse & WB $(1: 200)$ & home-production \\
\hline$\alpha$-actin & Mouse & WB $(1: 500)$ & Sigma \\
\hline phosphoAkt & Rabbit & IF $(1: 200)$ & $\begin{array}{l}\text { Cell Signalling, Danvers, } \\
\text { MA, USA }\end{array}$ \\
\hline $\mathrm{CNP}$ & Mouse IgG & IF $(1: 200)$ & Sigma-Aldrich \\
\hline GalC (clone MAB342) & Mouse IgG1 & IF $(1: 200)$ & $\begin{array}{l}\text { Millipore, Billerica, MA, } \\
\text { USA }\end{array}$ \\
\hline MBP & Mouse IgG1 & IF $(1: 1000)$ & $\begin{array}{l}\text { Sternberger, Lutherville, } \\
\text { MD, USA }\end{array}$ \\
\hline MBP & Rabbit & IF $(1: 300)$ & $\begin{array}{l}\text { DakoCytomat., Carpinte- } \\
\text { ria, CA, USA }\end{array}$ \\
\hline O1 & Mouse IgM & IF $(1: 50)$ & $\begin{array}{l}\text { (Kuhlmann-Krieg, 1988; } \\
\text { Sommer \& Schachner, } \\
\text { 1981) }\end{array}$ \\
\hline $\mathrm{PIP}_{2}$ & Mouse IgG2b & IF $(1: 100)$ & Echelon Bioscience \\
\hline $\mathrm{PIP}_{3}$ & Mouse IgM & IF $(1: 100)$ & Echelon Bioscience \\
\hline Phalloidin-Rhodamine & F-actin & IF $(1: 200)$ & $\begin{array}{l}\text { Invitrogen (Munich, Ger- } \\
\text { many) }\end{array}$ \\
\hline Phalloidin-Fluorescein & F-actin & IF $(1: 200)$ & $\begin{array}{l}\text { Invitrogen (Munich, Ger- } \\
\text { many) }\end{array}$ \\
\hline WGA-488 & leptin & IF $(1: 200)$ & $\begin{array}{l}\text { Invitrogen (Munich, Ger- } \\
\text { many) }\end{array}$ \\
\hline
\end{tabular}

\subsection{Specific Software}

List of specific software used for data acquisition, processing and analysis can be found in Table 2.2. The software/applications marked with $\dagger$ are freely available online.

Table 2.2: List of software

\begin{tabular}{|c|c|c|}
\hline Software & Application & Source/Manufacturer \\
\hline ImageJ† & image processing and analysis & http://rsbweb.nih.gov/ij \\
\hline Leica Confocal Software, 2.61 & acquisition of confocal images & $\begin{array}{l}\text { Leica Microsystems, Mannheim, } \\
\text { Germany }\end{array}$ \\
\hline MBF ImageJ for Microscopy $\dagger$ & image processing and analysis & http://wwwmacbiophotonics.ca/image \\
\hline Meta Imaging Series 6.1 & image processing and analysis & $\begin{array}{l}\text { Universal Imaging Corp., Down- } \\
\text { ingtown, USA }\end{array}$ \\
\hline GraphPad Prism & data quantification and statistical analysis & www.graphpad.com \\
\hline $\mathrm{LAT}_{\mathrm{EX}} \dagger$ & writing of the manuscript & www.latex-project.de \\
\hline Adobe Illustrator & formatting and compiling of figures & www.adobe-illustrator.softonic.de \\
\hline
\end{tabular}




\section{Methods}

\subsection{Cell culture}

All cell culture incubations were carried out in humidified $37^{\circ} \mathrm{C}, 7.5 \% \mathrm{CO}_{2}$ incubators; further culture conditions are specified below. All solutions and equipment coming into contact with living cells were sterile, all media were filter-sterilised with $0.22 \mu \mathrm{m}$ polyethersulfone (PES) filters (Millipore). Cell culture work was carried out according to security level S1 safety rules.

\subsubsection{Primary oligodendrocyte culture}

Primary cultures of oligodendrocytes were prepared from postnatal day 1 mouse brains as described previously (Fitzner et al., 2006; Simons et al., 2000; Trotter \& Schachner, 1989). In brief, cellular mixture from trypsinized mouse brains was grown in poly-L-lysine (PLL) coated flasks in Basal Medium Eagle (BME) medium supplemented with $10 \%$ horse serum and $100 \mathrm{U} / \mathrm{mL}$ each of penicillin and streptomycin. After 7 - 10 days, oligodendroglial progenitors growing on top of a layer of astrocytes were shaken off and cultured further in SuperSato medium (Table 2.3 on PLL-coated dishes or round coverslips $(\mathrm{d}=18 \mathrm{~mm})$ (R.Langenbrinck, Labor- und Medizintechnik, Emmendingen, Germany).

Table 2.3: SuperSato composition

\begin{tabular}{lll}
\hline B-27 Supplement & $50 \mathrm{X}$ & $2 \mathrm{~mL}$ \\
GlutaMAX-1 supplement & $200 \mathrm{mM}$ & $1 \mathrm{~mL}$ \\
Penicillin/Streptomycin & $5000 \mathrm{U} / 5000 \mu \mathrm{g}$ & $1 \mathrm{~mL}$ \\
Sodium pyruvate & $100 \mathrm{mM}$ & $1 \mathrm{~mL}$ \\
Triiodothyronine & $5 \mathrm{mM}$ stock in ethanol & $10 \mu \mathrm{l}$ \\
L-Thyroxine & $4 \mathrm{mM}$ stock in $0.26 \mathrm{~N} \mathrm{NaOH}, 25 \%$ ethanol & $13 \mu l$ \\
Horse serum & $100 \%$ & $1 \mathrm{~mL}$ \\
\hline
\end{tabular}

Prior to cell seeding, coverslips were washed for 1 hour with acid ( $\mathrm{HCl} \mathrm{37 \% ),}$ rinsed 3 times thoroughly with $\mathrm{ddH}_{2} \mathrm{O}$ and sterilised overnight at $260^{\circ} \mathrm{C}$. Cells were plated at density approximately $3 \times 10^{4}$ cells $/ \mathrm{cm}^{2}\left(7 \times 10^{4}\right.$ to $10^{5}$ cells per well in a 12-well plate).

Coating for primary cultures was prepared as following: PLL $(100 \mu \mathrm{g} / \mathrm{mL})$ was applied on the surfaces for $4-12 \mathrm{~h}$ at $37^{\circ} \mathrm{C}$ aspirated and washed with 1X PBS. 10X PBS was prepared according to (Sambrook \& Fritsch, 2001). To obtain 1X PBS, 10X PBS (Table 2.4) was diluted 10 times with $\mathrm{ddH}_{2} \mathrm{O}, \mathrm{pH}$ value was adjusted to 7.4 . 
Table 2.4: Phosphate Buffered Saline (PBS)

\begin{tabular}{ll}
\hline & \\
$\mathrm{NaCl}$ & $80.0 \mathrm{~g}$ \\
$\mathrm{KCl}$ & $2.0 \mathrm{~g}$ \\
$\mathrm{Na}_{2} \mathrm{HPO}_{4}\left(\right.$ or $\left.18.05 \mathrm{~g} \mathrm{Na}_{2} \mathrm{HPO}_{4} \times 2 \mathrm{H}_{2} \mathrm{O}\right)$ & $14.4 \mathrm{~g}$ \\
$\mathrm{KH}_{2} \mathrm{PO}_{4}$ & $2.4 \mathrm{~g}$ \\
& \\
\hline
\end{tabular}

\subsubsection{PtK2 fibroblast cell line}

PtK2 cell line was a courtesy of the NanoBiophotonics Department, Max Planck Institute for Biophysical Chemistry, Göttingen, Germany. Cells were grown in the flask with $10 \mathrm{ml}$ of the PtK2-Sato (Table 2.5). To prevent overgrowth, cells were splited once in $6-7$ days onto a fresh flask. For the imaging, cells were splited onto $18 \mathrm{~mm}$ coverslips (non-treated with acid and not-covered with PLL), in $1 \mathrm{ml}$ of PtK2-Sato.

Table 2.5: PtK2-Sato

\begin{tabular}{ll}
\hline DMEM & $80 \mathrm{ml}$ \\
FCS & $1 \%$ \\
Pen/Strep & $1 \%$ \\
Na pyruvat & $1 \%$ \\
\hline
\end{tabular}

\subsubsection{Nucleic acids delivery into cultured animal cells}

\section{Lipofectamin@ 2000 and TransIT@-based transfection}

Introduction of DNA into the cells, using Lipofectamin $囚 2000$ is based on forming liposomes with encapsulated DNA. Upon interaction with the cell membrane, liposomes can penetrate it and thus deliver the DNA of interest into the cell (Gregoriadis, 1984; Felgner et al., 1987). Exogenous nucleic acids were transiently introduced into primary oligodendrocyte cells by lipofection-based transfection method, following the manufacturer protocol. Cells were seeded on the $18 \mathrm{~mm}$ diameter coverslips in SuperSato medium and grown for the time needed. For each $18 \mathrm{~mm}$ coverslip the transfection mastermix of the following components were set in two test tubes: in one, $50 \mu \mathrm{l}$ of Opti-MEM@-I (Invitrogen, Carlsbad, CA, USA) were mixed with $4 \mu \mathrm{l}$ of Lipofectaminß 2000 (Invitrogen GmbH, Darmstadt, Germany) and $50 \mu \mathrm{l}$ Opti-MEM@-I were mixed with $1.6 \mu \mathrm{g}$ of purified plasmid DNA in another. Both mixtures were incubated for $5 \mathrm{~min}$ at room temperature. Next, solutions were combined and incubated further for $20 \mathrm{~min}$ at room temperature. Finally, the total volume of the prepared solution was added drop wise on the coverslip and cells were returned back to the incubator for 16-20 hrs.

To transfect cultured PtK2 cells, TransIT@ (Mirus Bio LLC, Madison, WI, USA) was used as transfection reagent. Cells with $75 \%$ confluence were preferably taken. For each coverslip in a 12-well plate, $3 \mu \mathrm{l}$ of transfection reagent and $1 \mu \mathrm{g}$ of plasmid DNA were mixed with $100 \mu \mathrm{l}$ Opti-MEM@-I and incubated $30 \mathrm{~min}$ at room temperature. The solution was added drop wise; cells were further kept under normal cultural condition and analysed after $16-20 \mathrm{hrs}$. 


\subsubsection{PtK2-MBP assay}

An assay of the reconstitution of the MBP zipping properties within fibroblast cell line was established (Aggarwal et al., 2013). In brief, the MBP-GFP-TM chimeric construct, containing MBP, transmembrane domain, GFP and ER-retention signal, (Figure 2.1) was designed and cloned. Subsequently, PtK2 cells were transfected with this construct and subjected to the further analysis after 18 hrs post-transfection. Cells, positive to the transfection, were recognised due to the GFP-signal.

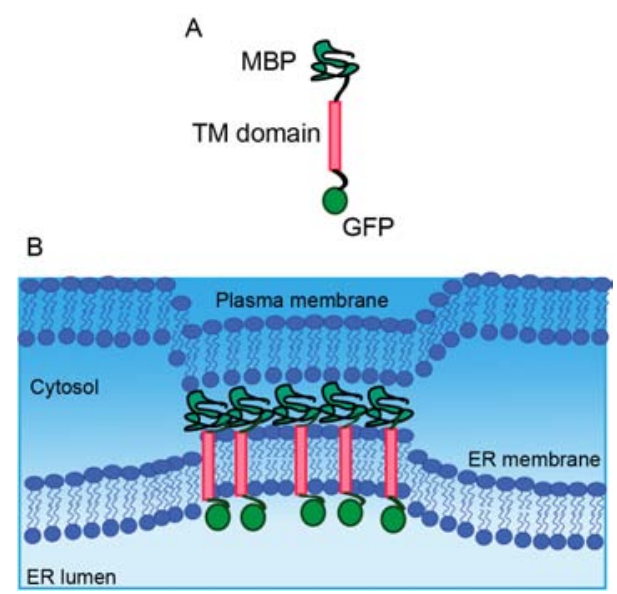

Figure 2.1: Schematic representation of the MBP-GFP-TM construct, used for the PtK2 transfection to reproduce MBP membrane zipping in the cell line. (A) Construct overview: MBP is fused to the transmembrane domain (TM), bearing endoplasmatic reticulum (ER)-retention signal, and to GFP. (B) Due to its TM domain MBP is anchored in the ER membrane, exposing its positive charge to the plasma membrane and with GFP molecule residing in the ER-lumen. Since PtK2 cells have flat morphology, ER membrane is in a close proximity to the cellular membrane. Hence, ER-hooked MBP is exposed to the negatively charged lipids of the plasmalemma and react with them, bringing two adjacent membranes (ER and cellular) together. These contacts are visualised due to the GFP signal.

\subsection{Inhibitors administration}

\subsubsection{PTEN inhibition}

VO-Ohpic, vanadium trihydrate (Biovision Research Products, Mountain View, CA, USA), was used as a specific PTEN inhibitor (Rosivatz et al., 2006; Nakai et al., 2004). It is referred to as VOOH in text and in figures. In general, VOOH was applied in the desired concentrations (200 pM, $100 \mathrm{nM}, 500 \mu \mathrm{m}, 1000 \mathrm{~nm})$ after 24 hrs of shake (day 1) with a repetitive addition of the same concentration on day 2. Cells were either fixed on day 3 , or allowed to grow till day 5 without inhibitor administration between day 3 and day 5 .

\subsubsection{Ionomycin treatment}

Ionomycin was used in stock of $10 \mathrm{mM}$. Krebs-Ringer solution for the Ionomycin treatment was prepared as described in Table 2.6. Cells were washed from the SuperSato 3 times with Krebs-Ringer $+\mathrm{Ca}^{2+}$. $10 \mu \mathrm{M}$ Ionomycin was applied for 
2 min in Krebs-Ringer $+\mathrm{Ca}^{2+}$ solution. 3 washing steps 1 min each with KrebsRinger $+\mathrm{Ca}^{2+}$ followed. Cells were further incubated for $1 \mathrm{~min}$ in saponin $(0.005 \%)$ (Sigma, S-7900) in Kebs-Ringer without $\mathrm{Ca}^{2+}$, and subsequently washed 3 times, again with $\mathrm{Ca}^{2+}$-free Krebs-Ringer. Cells were immediately fixed with $4 \% \mathrm{PFA}$, supplemented with $0.25 \%$ GTA and subjected to the immunocytochemistry.

Table 2.6: Krebs-Ringer solution

\begin{tabular}{lcc}
\hline Substance & Working & Stock \\
\hline $\mathrm{NaCl}$ & $120 \mathrm{mM}$ & $1.2 \mathrm{M}$ \\
$\mathrm{KCl}$ & $4.7 \mathrm{mM}$ & $47 \mathrm{mM}$ \\
glucose & $10 \mathrm{mM}$ & $100 \mathrm{mM}$ \\
$\mathrm{HEPES}$ & $20 \mathrm{mM}$ & $7.4 \mathrm{pH}$ \\
& & \\
adjust pH before adding last & & \\
two components & & \\
& & \\
\hline $\mathrm{CaCl}_{2}$ & $0.7 \mathrm{mM}$ & $0.5 \mathrm{M}$ \\
$\mathrm{MgSO}_{4}$ & & \\
To prepare Ca $^{2+}$-free & & \\
$\mathrm{Krebs}_{\text {Ringer, }}$ substitute $\mathrm{CaCl}_{2}$ & & \\
with equivalent amount of & & \\
$\mathrm{MgSO}_{4}$ & & \\
& & \\
\hline & & \\
saponin & $0.5 \%$
\end{tabular}

\subsection{Immunocytochemistry}

To analyse the subcellular localisation of the proteins and lipids of interest in primary oligodendrocytes and PtK2 cells we performed immunocytochemistry as described in (Simons et al., 2002). For common antibody staining, cells on coverslips were fixed for 10 min at RT with mixture of $4 \%$ paraformaldehyde (PFA) and $0.25 \%$ glutaraldehyde in PBS, pH 7.4, followed by three washing steps with $1 \mathrm{X}$ PBS. To access the internal structures, cells were permeabilised with $0.1 \%$ Triton X-100 for $1 \mathrm{~min}$ at RT and then washed 3 times in 1X PBS. For the surface staining this step was omitted. To reduce unspecific binding of antibodies, during internal protein staining, blocking was carried out for 30 min at RT in $2 \%$ FCS, $2 \%$ BSA and $2 \%$ fish gelatine in 1X PBS (100\% blocking solution). Cells were incubated with the primary antibodies in appropriate dilution in 10\% blocking solution for $1 \mathrm{~h}$ at room temperature, washed three times with $1 \mathrm{X}$ PBS, incubated with the secondary antibodies in required dilution in $10 \%$ blocking solution, washed three times in $1 \mathrm{X}$ PBS and mounted in Mowiol mounting medium. The list of antibodies used can be found in Table 2.1.

Exception: PIP $_{3}$ antibodies To analyse the subcellular localisation of $\mathrm{PIP}_{3}$ special protocol from manufacturer (Echelon) was applied, since the conventional one didn't provide a robust antibody signal. In brief, cells were fixed as mentioned above, washed 3 times with $1 \mathrm{X}$ TBS (Table 2.7) and permeabilise with 0,5\% saponin at room temperature for $15 \mathrm{~min}$, then again washed 3 times with TBS. Blocking was performed in $10 \%$ goat serum in TBS for $30 \mathrm{~min}$ at room temperature. Primary antibodies against $\mathrm{PIP}_{3}$ from Echelon were diluted in $1 \mathrm{X}$ TBS and applied for $60 \mathrm{~min}$ 
at room temperature. Coverslips were washed with $1 \%$ TBS-goat serum with subsequent staining with secondary $1 \mathrm{X}$ TBS-diluted antibodies. Cells were again washed 3 times in $1 \mathrm{X}$ TBS and mounted in Mowiol mounting solution.

Table 2.7: 1X Tris-buffered saline, TBS

$\begin{array}{lc}\text { Tris- } \mathrm{HCl} & 50 \mathrm{mM} \\ \mathrm{NaCl} & 150 \mathrm{mM} \\ \mathrm{ddH}_{2} \mathrm{O} & 1 \mathrm{~L} \\ \mathrm{pH} & 7.6\end{array}$

\subsubsection{Labelling with the small protein probes}

Small protein probes, consisting of the specific $\mathrm{PH}$-domain for the $\mathrm{PIP}_{2}$ (from $\mathrm{PLC}$ ) and $\mathrm{PIP}_{3}$ (from Grp1) recognition, labelled with either mCitrine or mCherry, respectively, were courtesy of Geert van den Bogaart, from Neurobiology department, Max Planck Institute for Biophysical Chemistry, Göttingen, Germany. Probes were applied onto fixed and permeabilised cells in 1:200 dilution in 1X PBS for $20 \mathrm{~min}$ at RT and afterwards thoroughly rinsed with 1X PBS (up to 10-15 times) (Khuong et al., 2013; van den Bogaart et al., 2011).

\subsubsection{Delivery of $\mathrm{PIP}_{3}$}

$\mathrm{PIP}_{3}$ was delivered to the cells via $\mathrm{PIP}_{3}$-shuttle kit from Echelon Bioscience Inc., following the manufacturers rules. In brief, $\mathrm{PIP}_{3}$, labeled with BODIPY-FL, was mixed with Histone H1 carrier (either labeled with TMR or non-labeled) in the 1:1 molar ratios (30 $\mu \mathrm{m}$ working concentration was used in the current experiments) for 15 minutes at RT. Formed complex was diluted to a desired working concentration with $100 \mu \mathrm{l}$ of SuperSato medium and applied on cells for 20 min at RT, using the wet chamber and sterile conditions. After incubation with $\mathrm{PIP}_{3}$, cells were transferred into fresh SuperSato and returned to the incubator. After $24 \mathrm{hrs}$ cells were fixed and immunolabelled. The positive delivery was trekked by a positive green signal from labelled BODIPY-FL-PIP ${ }_{3}$. Delivery in the control cells was controlled by a red signal from Histone H1, labelled with TMR-dye.

\subsection{Biochemistry}

\subsubsection{SDS-PAGE - Sodium Dodecyl Sulphate Polyacrylamide Gel Electrophoresis}

Polyacrylamide gels with ionic detergent sodium dodecylsulfate (SDS-PAGE) were used for the analytical electrophoresis of proteins (Laemli U.K., 1970). Proteins were separated due to their electrophoretic mobility in vertical electrophoresis system Bio-Rad Mini-PROTEAN (Bio-Rad Laboratories GmbH, München, Germany) in Tris-glycine buffer $(25 \mathrm{mM}$ Tris, $192 \mathrm{mM}$ glycine, $0.1 \%$ SDS ) for $2-3 \mathrm{~h}$ at $100 \mathrm{~V}$. Resolving and stacking gels composition are listed below (Table 2.8 and Table 2.9).

In general, prior the loading on the gel, samples were incubated at $60-65^{\circ} \mathrm{C}$ for $10 \mathrm{~min}$ in sample buffer in non-reducing conditions(Table 2.10). 
Table 2.8: Stacking gel for SDS-PAGE

\begin{tabular}{ll}
\hline & \\
Acrylamide/bis-acrylamide solution & $4 \%$ \\
Tris/HCl, pH 6.8 & $125 \mathrm{mM}$ \\
SDS & $0,1 \%$ \\
Ammonium persulfate (APS) & $0.05 \%$ \\
N'N'N'-tetramethylethylene diamine (TEMED) & $0.005 \%$ \\
\hline
\end{tabular}

Table 2.9: Resolving gel for SDS-PAGE

\begin{tabular}{ll}
\hline & \\
Acrylamide/bis-acrylamide solution & $12 \%$ \\
Tris/HCl, pH 8.8 & $375 \mathrm{mM}$ \\
SDS & $0.1 \%$ \\
Ammonium persulfate (APS) & $0.05 \%$ \\
N'N'N'-tetramethylethylene diamine (TEMED) & $0.005 \%$ \\
\hline
\end{tabular}

Table 2.10: Non-reducing sample buffer

\begin{tabular}{ll}
\hline & \\
Tris/HCl pH 6.8 & $50 \mathrm{mM}$ \\
Glycerol & $10 \%$ \\
EDTA & $2 \mathrm{mM}$ \\
SDS & $2 \%$ \\
Bromophenol blue & $0.05 \%$ \\
& \\
\hline
\end{tabular}

Molecular weights of the analysed proteins were estimated according to protein molecular weight, using a marker PageRuler R. Plus Pre-stained Protein Ladder (Fermentas, St. Leon-Rot, Germany).

\subsubsection{Western Blot}

Western blot analysis was used for identification and quantification of the proteins of interest. After SDS-PAGE separation, proteins were transferred onto Whatman R Protran Nitrocellulose Transfer Membrane (Whatman GmbH, Dassel, Germany). Transfer was performed for 1 hour, at $100 \mathrm{~V}$ in a transfer buffer (Table 2.11), in Bio-Rad Mini-Protein System (Bio-Rad Laboratories GmbH, München, Germany). Subsequently, membranes were subjected to immunological detection. To decrease the nonspecific binding, membranes were first incubated in the 5\% non-fat dried milk (Sigma-Aldrich Chemie GmbH, München, Germany) in PBST (PBS, as described in Table 2.4, supplemented with $0.1 \%$ Tween-20), for 30 min with mild agitation. Overnight incubation with primary antibodies with appropriate dilution in PBST, at $+4^{\circ} \mathrm{C}$ followed. Three washing steps in PBST, for 15 min each, were applied to remove the unbound antibodies, and secondary anti-mouse or anti-rabbit, HRPconjugated (horse reddish peroxidase) antibodies (Dianova, Hamburg, Germany), in 1:2000 dilution in PBST were applied for detection. Following $1 \mathrm{hr}$ at RT incubation, washing steps were repeated. The feature of HRP-enzyme, conjugated to the secondary antibodies, is the ability to excite luminol substrate by oxidising it; this results in a light emission. Therefore, labelled proteins on a membrane, were visualised by this chemiluminescence approach with Pierce ECL Western Blotting Substrate from Thermo Scientific (Epsom, United Kingdom). Developed X-ray films (CL-XPosureTM Film, ThermoScientific, Rockford, IL, USA) with detected chemiluminescent signals were scanned with a conventional scanner and the signal densities 
were quantified with ImageJ Gel Analysis plugin.

Table 2.11: Transfer buffer

\begin{tabular}{ll}
\hline Tris & $25 \mathrm{mM}$ \\
Glycine & $192 \mathrm{mM}$ \\
Methanol & $20 \%$ \\
& \\
\hline
\end{tabular}

\subsubsection{Quick Coomassie}

Visualisation of some of the SDS-PAGE was performed by "Quick Coomassie" protocol (Fairbanks et al., 1971). Solutions for this protocol are listed in Table 2.12. In brief, SDS-gels were boiled in the microwave for $30 \mathrm{sec}$ in each solution subsequently; each boiling followed by 5 min agitation on a shaker. The last washing step was done with the $\mathrm{ddH}_{2} \mathrm{O}$.

Table 2.12: Solutions for Quick Coomassie

\begin{tabular}{ll}
\hline Solution A & \\
Isopropanol & $750 \mathrm{ml}$ \\
Acetic acid & $300 \mathrm{ml}$ \\
Coomassie R-250 & $1,5 \mathrm{~g}$ \\
Total volume (with $\left.\mathrm{dH}_{2} \mathrm{O}\right)$ & $3 \mathrm{~L}$ \\
& \\
\hline & \\
Solution B & $300 \mathrm{ml}$ \\
Isopropanol & $300 \mathrm{ml}$ \\
Acetic acid & $150 \mathrm{mg}$ \\
Coomassie R-250 & $3 \mathrm{~L}$ \\
Total volume (with $\left.\mathrm{dH}_{2} \mathrm{O}\right)$ & \\
& \\
\hline & \\
Solution C & $300 \mathrm{ml}$ \\
Acetic acid & $60 \mathrm{mg}$ \\
Coomassie R-250 & $3 \mathrm{~L}$ \\
Total volume (with $\left.\mathrm{dH}_{2} \mathrm{O}\right)$ & \\
\hline & \\
\hline $\begin{array}{l}\text { Solution D } \\
\text { Acetic acid } \\
\left.\text { Total volume (with d } \mathrm{H}_{2} \mathrm{O}\right)\end{array}$ & $300 \mathrm{Ll}$ \\
& \\
\hline
\end{tabular}

\subsubsection{Preparation of large unilamellar vesicles (LUVs) and their pull-down with MBP}

To investigate binding preferences of MBP to phosphatidylinositols in vitro, method of liposomal pull-down was used. Liposomes were generated with the following composition: PC:PE, PC:PS, PC:PIP 2 , PC:PIP ${ }_{3}$, with the 80 to 20 ratio. All lipids (except of $\mathrm{PIP}_{3}$ ) were purchased from Avanti Polar Lipids Inc. $\mathrm{PIP}_{3}$ was acquired from Echelon Bioscience Inc. Preparation of LUVs was done as described in (Aggarwal et al., 2011). In brief, lipids were taken in the appropriate molar ratios and mixed in $500 \mu \mathrm{l}$ of chloroform. The solvent was then evaporated in a Speed-Vak centrifuge for $2 \mathrm{hrs}$, at $30^{\circ} \mathrm{C}$. Dried films were further resuspended in LUVs buffer (50 mM HEPES, $100 \mathrm{mM} \mathrm{NaCl}, \mathrm{pH}$ 7.4) to a $1 \mathrm{mg} / \mathrm{ml}$ concentration and extensively 
vortexed. Translucent solution was further sonicated on the water bath for 15-20 minutes, at $70^{\circ} \mathrm{C}$, until the solution became transparent. On this step the multilamellar vesicles (MUVs) were generated. To receive the large unilamellar vesicle from this mixture, the Avanti Polar Lipid Extrusion protocol was applied. MUVs were subjected to 5 freeze/thaw cycles (liquid nitrogen $/ 60^{\circ} \mathrm{C}$ bath, respectively) with a subsequent 10 extrusion cycles through the extruder (Avanti Polar Lipid manufacturer protocol). LUVs with $1000 \mathrm{~nm}$ size were generated. The size was controlled via pore diameter of the filter used in the extrusion procedure. Prepared LUVs were mixed with 14kDa recombinant MBP (purified by Steffen Frey, Cellular logistics department, Max Planck Institute for Biophysical Chemistry, Göttingen, Germany) and incubated for 10 min RT, until a precipitates were visible. Mixture was then spinned down at the maximum speed for $15 \mathrm{~min}$. Supernatant and pellet were applied as separate samples on SDS-PAGE gel and visualised after gel run by Quick Coomassie protocol. Band density quantifications were performed using ImageJ Gel Analysis plugin.

\subsubsection{Soluble/insoluble actin fractionation}

To determinate the amount of filamentous (F) and globular $(\mathrm{G})$ actin within cells the method of soluble/insoluble actin fractionation was used (Papakonstanti, 2007). In brief, cells, grown on the coverslip for 3 or 5 days, were incubated with $500 \mu \mathrm{l}$ of Triton-buffer for $5 \mathrm{~min}$ on ice. Supernatant with soluble proteins was collected. Next, cells were scrapped from the coverslip in $500 \mu \mathrm{l}$ RIPA-buffer. Any remaining insoluble material was removed by centrifugation (max speed, 10 min). Supernatants were collected and subsequently processed with SDS-PAGE and Western Blot analysis. Anti-actin mouse IgG primary antibodies were used for the both fractions recognitions. Quantification of the density bands was performed with ImageJ Gel Analysis plugin. For the Triton- and RIPA-buffer solutions see Table 2.13 and Table 2.14.

Table 2.13: Triton-extraction buffer

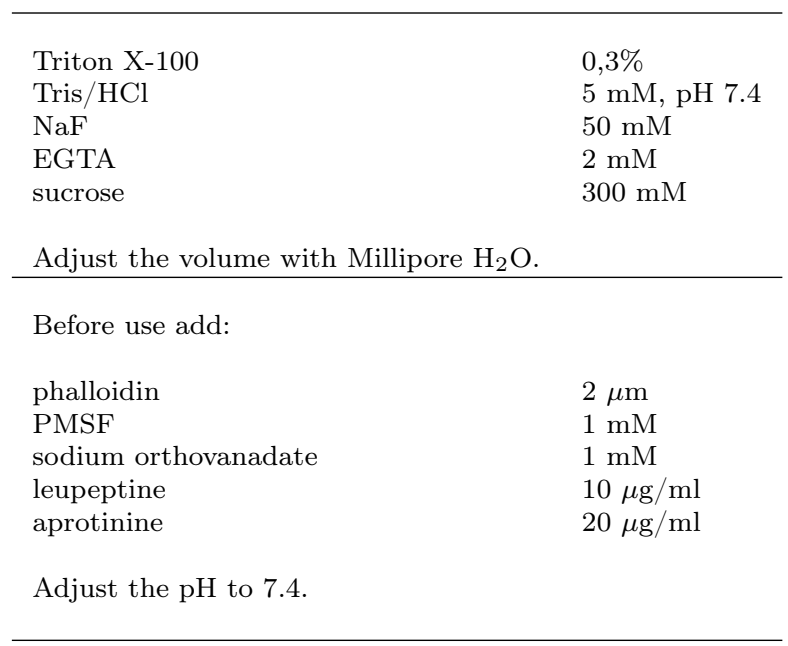

\subsubsection{BSA-coupling of artificial lipids}

To enable incorporation into cellular membranes, lipids of interest were primarily coupled to bovine serum albumin (BSA) as described in (Martin \& Pagano, 1994) 
Table 2.14: RIPA-extraction buffer

\begin{tabular}{ll}
\hline & \\
Triton X-100 & $1 \%$ \\
Tris $/ \mathrm{HCl}$ & $50 \mathrm{mM}, \mathrm{pH} 7.4$ \\
sodium deoxycholate & $1 \%$ \\
$\mathrm{SDS}$ & $0.1 \%$ \\
$\mathrm{NaCl}$ & $0.15 \mathrm{M}$ \\
$\mathrm{EDTA}$ & $1 \mathrm{mM}$ \\
& \\
Adjust the volume with Millipore $\mathrm{H}_{2} \mathrm{O}$. & \\
& \\
\hline & \\
Before use add: & \\
sodium orthovanadate & $1 \mathrm{mM}$ \\
DTT & \\
Adjust the pH to 7.4. & \\
\hline
\end{tabular}

with slight modifications. Lipids were first reconstituted in desired concentrations in the chloroform/methanol solution; liquid was further aspirated to create a lipid films. $75 \mathrm{nmol}$ lipid films were redissolved in $10 \mu \mathrm{l}$ of absolute ethanol and vortexed vigorously. $1 \mathrm{ml}$ of defatted BSA (in Dulbecco's Modified Eagle Medium DMEM without phenol-red, buffered with $10 \mathrm{mM}$ HEPES) was added in equimolar concentration to the lipids. Solution was centrifuged at the maximum speed for 3 min to remove any clumps. Supernatant was stored at $-20^{\circ} \mathrm{C}$.

\subsubsection{Incorporation of the artificial lipids}

To incorporate lipids into cellular membranes, cells were first washed with the $10 \mathrm{mM}$ HEPES-buffered DMEM medium without phenol-red (HDMEM, ice-cold). Next, BSA-lipid complexes, diluted in HDMEM, were added in the appropriate concentrations onto cells in the wet-chamber on ice for $30 \mathrm{~min}$. After incubation, cells were briefly washed with ice-cold HDMEM and immediately imaged to prevent further internalization of lipids. The fluorescent lipophilic organic dye Atto647N (excitation max at $645 \mathrm{~nm}$, emission max at $670 \mathrm{~nm}$; Atto-Tec, Siegen, Germany) was used as a marker of the incorporated lipids (Eggeling et al., 2009). Cerebroside C23:0 was labelled with KK114 dye.(red emission, Rhodamine family (Wurm et al., 2012). Lipids, used for the incorporation and diffusion measurements are listed in Table 2.15 .

Table 2.15: Artificial lipids for STED-FCS

\begin{tabular}{llll}
\hline Label & Name & Labelling type & Reference in text \\
\hline Atto647N-PE & $\begin{array}{l}N \text {-(Atto647N)-1,2-dihexadecanoyl-sn- } \\
\text { glycero-3-phosphoethanolamine } \\
N \text {-(Atto647N)-sphingomyelin }\end{array}$ & head group labelling & PE \\
Atto647N-SM & $\begin{array}{l}N \text {-(Atto647N)-galactosylsphingosine } \\
\text { Atto647N-GalC } \\
\text { Cerebroside C23:0 KK114 }\end{array}$ & $\begin{array}{l}\text { labelling by acyl-chain } \\
\text { labelling by acyl-chain } \\
\text { replacement } \\
\text { as 3rd chain labelling }\end{array}$ & GalC \\
\hline
\end{tabular}

Lipids were labelled either at the head group (PE), meaning at the water phase or lipid-water interphase, or via replacement of one the native lipid acyl chains by 
the short acyl chain carrying a dye (acyl-chain replacement - SM, GalC). In case of 23C-GalC, label was introduced as a third chain. Throughout the text these lipids will be refereed simple as PE, SM, GalC and 23C-GalC. All aforementioned lipids are the courtesy of the C. Eggeling group from the NanoBiophotonics department, Max Planck Institute for Biophysical Chemistry, Göttingen, Germany.

\subsection{Microscopy}

\subsubsection{STED-FCS Microscopy}

\section{STED-FCS: theory behind}

Fluorescence correlation spectroscopy is a powerful method of time-averaging fluctuation analysis that is sensitive and statistically robust. Recorded fluctuations arise due to the behaviour of the trespassing molecules within the illuminated focal area. These averaged fluctuations $\delta \mathrm{F}$ of the recorded signal $\mathrm{F}(\mathrm{t})$ are autocorrelated, giving the normalised fluctuation autocorrelation function $\mathrm{G}\left(\tau_{c}\right)$ (Magde, 1974).

$$
G\left(\tau_{c}\right)=<\delta F(t) \times \delta F(t+\tau)>/\left[F(t)^{2}\right]
$$

Here $\tau_{c}$ represents a correlation lag time and triangular brackets indicate averaging over measurements time $t$.

FCS data recorded for free two-dimensional Brownian lipid diffusion would be described by following model:

$$
G_{D}\left(t_{c}\right)=\left(1+\left(t_{c} / \tau_{D}\right)\right)^{-1}
$$

with

$$
\tau_{D}=d^{2} /(8 \ln 2 D)
$$

Here, $\tau \mathrm{D}$ is the average transit time of a fluorophore with an apparent diffusion coefficient $\mathrm{D}$ through the observation area of diameter $d$. The decrease of the diameter of the focal area also contributes to the precise data accumulation with FCS method: the smaller the observation field, the higher chances to distinguish trapping of the molecule from their slow, but free diffusion. Confocal FCS set-ups are of course limited in this regard, but this is where a potent STED (stimulated emission depletion microscopy) technique comes into play.

The main principle of STED nanoscopy lies in th reversible inhibition of the fluorescence emission of a fluorophore by high power stimulated emission, typically at the red edge of the spectrum. STED light emission inhibits the fluorescence everywhere around, except the lone spot within the doughnut-shaped laser beam (Hell \& Wichmann, 1994; Hell, 2009). Changing the STED power, one can modify the diameter of the doughnut center, therefore changing the size of the effective fluorescent region.

In our study we investigated the mode of lipid diffusion within the myelin membrane. To elucidate this question, we used a combined STED-FCS technique that allows to go below conventional diffraction barrier and distinguish diffusion behaviour on the nanoscale.

In the STED-FCS approach, data of the lipid diffusion are collected for different STED powers $P$-STED, meaning for different diameters $d$ (Mueller et al., 2013). To 
deal with the anomalous diffusion in the FCS model an anomalous diffusion exponent $\alpha$ is introduced into the equation 2.4 (Wachsmuth et al., 2000).

$$
G_{D}\left(t_{c}\right)=\left(1+\left(t_{c} / \tau_{D}\right)^{\alpha}\right)^{-1}
$$

with

$$
\tau_{D}=d^{2} /(8 \ln (2) D)
$$

Here, again, $\tau \mathrm{D}$ is the average transit time of a fluorophore with the apparent diffusion coefficient D through the focal detection area of diameter $d$. In case of free Brownian motion, $\mathrm{D}$ is constant for different $d$, therefore $\tau \mathrm{D}$ has a linear dependence with the focal area $d^{2}$. However, an event of anomalous diffusion brings a variation in $\mathrm{D}$ with $d^{2}$. This variation depends on the spatial and temporal characteristics of the anomalous diffusion trigger (time of the trapping, size of the obstacle, nature of the interactions, etc). Moreover, one can further extract information and parameters to describe anomalous diffusion, in addition to the anomaly parameter $1 / \alpha$. Upon calibration of the STED-FCS set-up for the lipid marker Atto647N by SSLBs measurements, the dependence between the diameter $d$ of the focal area and STED powers $(P)_{S T E D}$ is known. Fitting correlation data into equation 2.4, one can obtain the average transit time $\tau \mathrm{D}$ and plot it against the focal area $d^{2}$ (Wawrezinieck et al., 2005). Linear relationship would represent free Brownian diffusion. Any fluctuations from the linear dependence would indicate an event of trapping, resulting in the decrease of $\tau \mathrm{D}$ with $d^{2}$ and a concave function of $d^{2}$. In contrary, hindered diffusion within a meshwork or hopping-like diffusion would show an increase in the $\tau \mathrm{D}$ with $d^{2}$ and a convex function of $d^{2}$ (Wawrezinieck et al., 2005; Lenne et al., 2006; Mueller et al., 2013).

\section{STED-FCS experimental setup}

The STED nanoscope was based on a home-built confocal microscope setup equipped with a $640 \mathrm{~nm}$ laser (100 ps pulse width, LDH-D-C-640, PicoQuant, Berlin, Germany) for excitation of the Atto647N dye (ATTO-TEC). The STED beam was provided by a Titanium:Sapphire laser system (Chameleon, Coherent Inc.) operating at $780 \mathrm{~nm}$ with a repetition rate of $90 \mathrm{MHz}$. The time interval between the pulses of both lasers was adjusted using a home-built electronic delay unit, where the STED pulses served as the trigger master. The STED laser pulses were stretched from 200 fs to a pulse length of approximately 180 ps using four $30 \mathrm{~cm}$ optical SF6 glass rods and a $125 \mathrm{~m}$ long polarisation maintaining single-mode fibre (AMS Technologies). Fluorescence excitation and collection was done using an oil immersion objective (APON 60x, NA=1.49, Olympus). The laser beams were spatially overlaid and the fluorescence light filtered by dichroic filters (AHF Analysentechnik). The doughnut-shaped focal spot of the STED beam featuring a central zero intensity was produced by introducing a phase-modifying plate (RPC Photonics) into the beam path, imprinting on the wave front a helical phase $\operatorname{ramp} \exp (\mathrm{i} \phi)$ with $0 \leq \phi \leq 2 \pi$. A $\lambda / 4$-plate ensured circular polarisation of the STED and excitation beams. The fluorescence was coupled into a multi-mode fibre splitter (Fibre Optic Network Technology) with an aperture size corresponding to $1.4 \mathrm{x}$ the magnified excitation spot. The 50:50 split fluorescence signal was then detected by two single-photon counting modules (avalanche photo diode SPCM-AQR-13-FC, Perkin Elmer Optoelectronics) 
and the recorded fluorescence counts were further processed by a hardware correlator card (Flex02-01D, Correlator.com). The focal intensity distribution of the excitation and STED light were measured by scanning a scattering gold bead of $80 \mathrm{~nm}$ in diameter (gold colloid, En.GC80, BBinternational) using a non-confocal detector (MP 963 Photon Counting Module, Perkin Elmer). The laser powers $\mathrm{P}$ were measured directly at the sample plane. Together with the full-width-at-half-maximum FWHM (corresponds to effective beam diameter) of the focal laser intensity distribution $(\approx 240 \mathrm{~nm}$ for $640 \mathrm{~nm}$ wavelength) they allow for the calculation of the time-averaged intensity, I (see Eq. 2.6, usually $\approx 22 \mathrm{~kW} / \mathrm{cm}^{2}$ stemming from $\mathrm{P}=10 \mu \mathrm{W}$ for the excitation light and $\approx 309 \mathrm{MW} / \mathrm{cm}^{2}$ stemming from $\mathrm{P}=140 \mathrm{~mW}$ for the highest STED power.

$$
I=P /\left[\pi(F W H M / 2)^{2}\right]
$$

\section{STED-FCS calibration}

Supported lipid bilayers (SLBs) were used to calibrate the STED-FCS setup. The SLBs were prepared based on a procedure described elsewhere (Mennicke \& Salditt, 2002). Briefly, the lipid DOPC (1,2-dioleoyl-sn-glycero-3-phosphocholine, Avanti) and a fluorescent lipid analogue (DPPE-Atto647N) were mixed in organic solvents (Chloroform/MeOH 1:1) at a lipid concentration of $1 \mathrm{mg} / \mathrm{ml}$. The ratio of labelled lipids per non-labelled ones was approximately 1:10000. $50 \mu \mathrm{l}$ of such solution were dropped onto a plasma-cleaned (Femto-RF, Diener Electronic) standard microscope cover glass (diameter $22 \mathrm{~mm}$, no. 1.5 thickness) and spin-coated at $60 \mathrm{~Hz}$ for about one minute. The cover glass was then placed in a microscopy chamber and subsequently the dry thin lipid film was rehydrated with $500 \mu \mathrm{l}$ buffer solution $(150 \mathrm{mM}$ $\mathrm{NaCl}, 10 \mathrm{mM}$ HEPES). Such bilayers were stable for several hours.

Calibration of the diameter $\mathrm{d}\left(\mathrm{P}_{S T E D}\right)$ of the effective focal fluorescence spots formed by a certain STED power $\mathrm{P}_{S T E D}$ was performed by STED-FCS measurements of fluorescent lipid analogues in supported lipid bilayers (SLBs), for that such lipid bilayers provide a two-dimensional free diffusing system of molecules, labelled with the same fluorophore used in our cellular experiments. The confocal FWHM being determined by fluorescent beads $\left(\mathrm{d}\left(\mathrm{P}_{S T E D}=0\right) \approx 240 \mathrm{~nm}\right)$, the other effective diameters $\mathrm{d}\left(\mathrm{P}_{\text {STED }} \neq 0\right)$ can be calculated by performing STED-FCS measurements on supported lipid bilayers and using the relation:

$$
\frac{d\left(P_{S T E D}=0\right)}{d\left(P_{S T E D}\right)}=\sqrt{\frac{\tau D\left(P_{S T E D}=0\right)}{\tau D\left(P_{S T E D}\right)}}
$$

In Eq. 2.7, $\tau \mathrm{D}$ stands for the transient times correspondent to each given $\mathrm{P}_{S T E D}$. The relation above stems from the hypothesis that the lipids in SLBs undergo absolute two-dimensional free diffusion, so that the diffusion time scales proportionally with the diffusion area.

\section{STED-FCS cellular measurements}

Day 5 oligodendrocytes and PtK2 cells were grown on standard glass coverslips (diameter $18 \mathrm{~mm}$, no. 1.5 thickness) to a confluence of about $80 \%$ at $37^{\circ} \mathrm{C}$ in a water-saturated atmosphere of $5 \% \mathrm{CO}_{2}$ in the air. Incorporation of the fluorescent 
lipid analogues (lipid-Atto647N) by cells was accomplished via BSA coupling. Measurements were performed at $23^{\circ} \mathrm{C}$ in HDMEM.

We assessed the dynamics of fluorescent lipid analogues by placing the focused cocentred excitation and STED beams on random positions at the plasma membrane and completed all measurements before any significant morphological changes in the cell could occur. All measurements were carried out during $10 \mathrm{~s}$, providing a correlation time longer than two orders of magnitude times the typical transient time of the labelled lipids through the confocal observation area. The calculated apparent diffusion coefficient for each given effective beam diameter resulted from the average of measurements performed in different cells. For each cell analysed, the calculated apparent diffusion coefficient for each given effective beam diameter, resulted from the average of at least 2 and up to 8 repetitions of the STED-FCS measurement at the given cell. For each given effective beam diameter, the averaged values obtained from individual cells were then averaged so that the correspondent s.e.m. reflects the variance among cells.

\section{STED-FCS analysis}

Correlation data were recorded with lipid concentrations resulting in a temporal average of particle number $\mathrm{N} \approx 0.5-10$ fluorescent particles for the highest STED depletion and $\mathrm{N} \approx 10-200$ fluorescent particles for confocal recording. We fitted all correlation data with a model for two-dimensional (possibly anomalous) diffusion, resulting in different values of the average transient time $\tau \mathrm{D}$ and anomaly parameter $\alpha$. Free diffusion corresponds to $\alpha=1$, while anomalous diffusion displays lower values of $\alpha$. Nevertheless, bad fits may also result in low $\alpha$, reason why we systematically discarded the infrequent data $(<5 \%)$ that was fitted with $\alpha<0.50$ (pre-established criterion). Each individual measurement resulting in a transient time $\tau \mathrm{D}$, the correspondent diffusion coefficient $\mathrm{D}$ was calculated based on the knowledge of the effective beam diameter, provided by the calibration of the system:

$$
D=d^{2} /(8 \tau D \ln (2))
$$

\subsubsection{Confocal Microscopy}

For morphological examination of myelin membrane sheets produced by primary oligodendrocytes we chose laser scanning confocal microscopy. This technique gives an advantage of high resolution in combination with control over depth of field, which is invaluable for the analysis of organisation of thin membrane sheets.

Confocal microscopic images were acquired with Leica DMIRE2 microscope and a Leica TCS SP2 AOBS confocal laser scanning setup (Leica Microsystems, Mannheim, Germany). 40X NA 1.25 or 63X NA 1.4 oil plan-apochromat objectives (Leica Microsystems, Mannheim, Germany) were used for image acquisition.

\subsection{Image Processing and Statistical Analysis}

Images were processed and analysed with the public domain Java-based image processing software ImageJ (created by Rasband, W.S., National Institutes of Health, 
Bethesda, Maryland, USA), or with an MBF collection of plugins for ImageJ ("MBF ImageJ for Microscopy").

Quantification of relative cell surface area occupied by the myelin proteins oligodendrocytes was performed using the following options in ImageJ: application of threshold from background (with the Segmentation Plugin to avoid user-bias from manual setting of the threshold (Chen \& Leung, 2004)), defining cell area (Freehand selection tool), followed by measurement of the area fraction, occupied by the signal (Set measurements - Area fraction - Measure).

SDS-PAGE gels, stained with Coomassie and Western blot membranes densitometric analysis was carried out on the blot and gel scans with the ImageJ Gel Analyser plugin: Analyse - Gels - Select lanes - Plot lanes - Measure peak areas.

Statistical analysis was performed using GraphPad Prism software (Table 2.2): two-tailed, unpaired t-test and One-way ANOVA with Tukey post-hoc test. 


\section{RESULTS}

No amount of experimentation can ever prove me right; a single experiment can prove me wrong.

Albert Einstein 



\section{Part I: Lipid dynamics and organisation in the myelin membrane sheets}

This part of my thesis presents results from investigation of lipid behaviour within oligodendrocyte sheets. Since myelin in vivo is a system of the wrapped membrane layers around the axon, assessment of a single-layered membrane becomes challenging. In our studies we used a well-established 2D model of the myelin sheath - cultured oligodendrocytes (Aggarwal et al., 2011). Mouse oligodendrocytes are grown on coverslips and expand their sheath-like structures (sheets) that are easy to study with microscopy methods.

\subsection{Cultured oligodendrocytes as a model system}

Primary cell culture was extensively used in this work. With respect to the mature oligodendrocyte morphology, two major subcellular domains were distinguished: cell body together with the processes and most outer part of the cell (referred to as rim) and thin flat membrane expansions in between the processes, referred to as sheets. 2/,3/,-cyclic-nucleotide-phosphodiesterase (CNP) was used as a marker for the former domain, and myelin basic protein (MBP) for the latter one (Figure 3.1). The day of the seeding (shake) is referred to as day 0. We further classify cells, based on their morphological appearances. On day 1 after shake the majority of cells on the coverslip are represented by oligodendrocyte precursor cells (OPC) with only several brunched processes and no sheets (Figure 3.1, A1-A3). After three days in culture (day 3), majority of the cells on the coverslip have CNP-positive sheets in between the brunched processes. MBP-positive regions start to appear at this stage. Cells at this stage are referred to as immature and they are characterised morphologically by a large rim. This is a stage of the active growth of the oligodendrocytes (Figure 3.1, B1-B3). After five days in culture (day 5), majority of the cells have MBPpositive compacted sheets, with CNP being present only in processes and outer rim. Processes become less brunched. Cells on this stage are referred to as the mature. They are characterised morphologically by a small rim and decrease in the number of processes(Figure 3.1, C1-C3). 


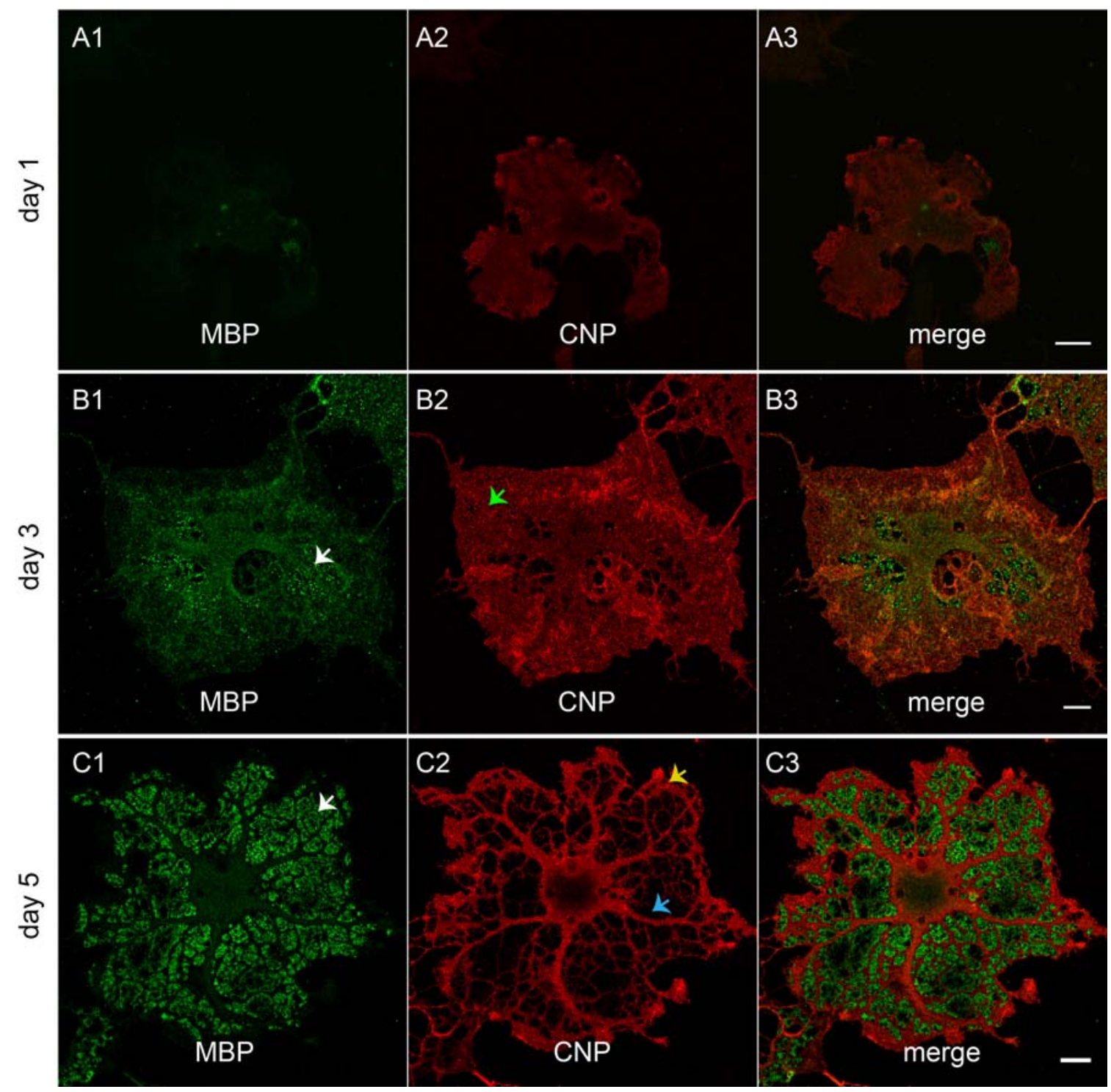

Figure 3.1: Domains of oligodendrocytes, cultivated in vivo. Cultured oligodendrocytes were fixed with $4 \%$ paraformaldehyde and $0.25 \%$ glutaraldehyde (PFA $4 \%+$ GTA 0.25 $\%$ ) on day 1 (A1-A3), day 3 (B1-B3) and day 5 (C1-C3). After fixation cells were incubated with anti-MBP antibodies (in green - A1, B1 and C1) to visualise a start of the MBP expression (B1, white arrowhead) and compact membrane sheets (C1, white arrowhead). Anti-CNP antibodies were used to visualise non-compact sheets on day 1 (red, A2), non-compact regions on day 3 (red, $\mathrm{B} 2$, green arrowhead) and non-compact processes ( $\mathrm{C} 2$, blue arrowhead) and outer rim (C2, yellow arrowhead) on day 5 . Scale bar $=10 \mu \mathrm{m}$.

\subsection{Unique features of myelin membrane sheath that influence lipid behaviour}

In contrast to artificial membranes (SSLBs, liposomes, black membranes) where lipid diffusion is free, in the plasma membrane lipid diffusion might exhibit anomalous behaviour. Anomalous diffusion in the cellular membrane occurs due to the proteinprotein interactions, confinements of the protein cytoplasmic tails by cytoskeleton and self-organisation of the lipids into the microdomains with changed membrane order and viscosity (Owen et al., 2009). Membrane lipids show different behaviour 
throughout a cellular membrane. This behaviour is classified into free diffusion, transient trapping and hopping. It was shown that cortical actin has a large influence on lipid dynamics in the membrane, however this impact is mostly indirect. Actin anchors various transmembrane proteins by their cytoplasmic tails and aligns them along the actin cytoskeleton, thus creating rows of pickets against the free diffusion of lipids. Moreover, when transmembrane proteins get immobilised, the viscosity of the membrane around it becomes higher, due to hydrodynamic friction effects at the surface of the immobilised protein. Therefore, when there is a high number of anchored transmembrane proteins aligned along the actin cytoskeleton, this contributes to the changes in the lipid behaviour (Kusumi \& Suzuki, 2005).

Additionally, lipid diffusion was shown to depend on cholesterol level in the membrane (Eggeling et al., 2009; Mueller et al., 2011). The lipid-ordered phase, created by cholesterol-rich domains, dramatically decreases free diffusion of lipids, enhancing lipid trapping, in comparison to the disordered phase, albeit for short distances $(<20$ $\mathrm{nm}$ ) and for short periods of time (10-20 ms) (Eggeling et al., 2009).

Studies of lipid diffusion were performed on different cell types, all of them, however, sharing a very common membrane composition. Myelin lipid composition is very distinct from the usual cellular membrane. Myelin membrane has an increased number of highly saturated long-chain fatty acids together with high cholesterol amount. In addition, protein number in myelin is very scarce with the ratio of $80 \%$ of lipids and only $20 \%$ of proteins with myelin basic protein (MBP) and proteolipid protein (PLP) being the most prominent ones. Additional feature comes from myelin development: upon maturation the amount of the filamentous actin dramatically drops, leaving an adult myelin membrane without any cortical actin cytoskeleton underneath. Role of MBP as an extruder that creates this specific protein-poor and lipid-enriched membrane where cytoskeleton meshwork is absent was extensively discussed (Aggarwal et al., 2013; Aggarwal et al., 2011; Yurlova et al., 2011).

Dynamical aspects of the lipids within myelin sheath haven't been addressed before. Therefore, the aim of this part of my PhD project was to shed some light on the lipid behaviour in this specific environment.

The most powerful method to resolve modes of diffusion and diffusion coefficients with high precisions is fluorescence correlation spectroscopy (FCS), combined with stimulated emission depletion microscopy (STED) (see Section 2.8.1 in Methods) (Eggeling et al., 2009; Sahl et al., 2010). The limitation of resolution to $250 \mathrm{~nm}$ due to the diffraction limit restricts confocal microscopy from the differentiation between free and hindered diffusion of molecules on small scales. However, the subdiffraction spot, created by STED beam on top of the confocal focal spot makes it possible to study lipid diffusion within a subdifraction spot. Assuming that freely moving molecule dwells only shortly within the STED detection area, whereas trapped molecules stay longer, it is possible to precisely distinguish restricted movements from free diffusion.

PtK2 fibroblast cell line was taken as a control cellular system for our studies. Lipid dynamics and behaviour were thoroughly characterised in these cells elsewhere (Eggeling et al., 2009; Mueller et al., 2011). It was shown, for instance, that actin and cholesterol amount have a major influence on the lipid diffusion in these cells. 


\section{Diffusion coefficient for PE, SM and GalC in control PtK2 cells and oligodendrocytes}

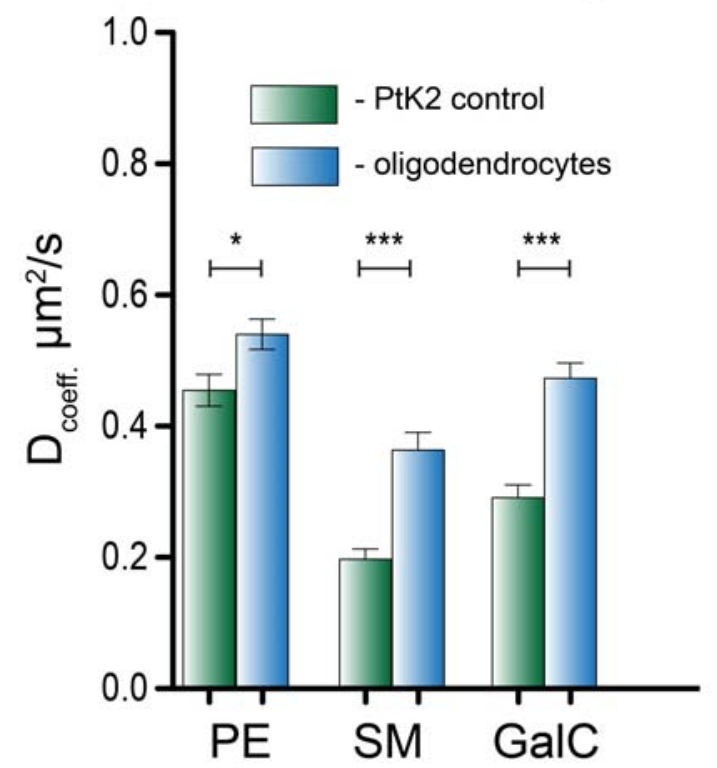

Figure 3.2: Diffusion coefficient of the PE, SM and GalC in oligodendrocytes is higher than in PtK2 cells. Cultured oligodendrocytes on day 5 were incubated with a mixture of BSAcoupled artificial lipids labelled with Atto647N. After 30 minutes incubation with BSA-coupled lipids, oligodendrocytes were subjected to a STED-FCS. PtK2 control cells were subjected to the same procedure. Diffusion coefficients were measured at the smallest effective STED beam diameter ( $\mathrm{d}=40 \mathrm{~nm}$, highest STED power) for the PE, SM and GalC for PtK2 (green) cells and oligodendrocytes (blue). Diffusion coefficient for PE is very similar in both cellular systems, although it is slightly higher in oligodendrocytes . GalC and SM diffusion is faster in oligodendrocytes, than in PtK2 cells. $\mathrm{N}=20$ cells, error bars represent s.e.m.; ${ }^{*}-\mathrm{p}<0.05,{ }^{* * *}-\mathrm{p}<0.0001$, t-test.

\subsection{Lipid diffusion in oligodendrocyte sheets versus PtK2 cell membrane}

To determine the dynamical properties of the lipids (diffusion coefficient and mode of diffusion) within the oligodendrocyte sheet we used the following Atto647Nlabelled artificial lipids, as analogs of the endogenous membrane lipids:

- $N$-(Atto647N)-1,2-dihexadecanoyl-sn-glycero-3-phosphoethanolamine as an analog of phosphatidylethanolamine (referred to as PE);

- $N$-(Atto647N)-sphingomyelin as an analog of sphingomyelin (referred to as $\mathrm{SM})$;

- $N$-(Atto647N)-galactosylsphingosine as an analog of galactosylcerebroside (referred to as GalC).

These lipids were introduced into the membrane via BSA-coupled method, see Section 2.7.6 in Methods. Measurements of the dynamical characteristics where taken on the STED-FCS setup and analysed in collaboration with D. M. Andrade (department of NanoBiophotonics, C. Eggeling group, Max Planck Institute for Biophysical Chemistry, Göttingen, Germany). 
To assess the mode of lipid diffusion, we measured the diffusion coefficient for the highest STED power $\left(\mathrm{D}_{S T E D}\right)$ at effective beam diameter $(\mathrm{d})$ of the observation area of $40 \mathrm{~nm}$. As it is illustrated in the Figure 3.2, values for the diffusion coefficient of PE in PtK2 cells and oligodendrocytes, measured at the highest STED power (that is with the smallest observation area for the molecule to cross), are similar: $0.48 \mu \mathrm{m}^{2} / \mathrm{s} \pm 0.05$ s.e.m. for the PtK2 cells and $0.51 \mu \mathrm{m}^{2} / \mathrm{s} \pm 0.05$ s.e.m. for the oligodendrocytes. However, values for the diffusion coefficient for SM and GalC are strikingly different between the two cell types: slow diffusion for the SM $(0.19$ $\mu \mathrm{m}^{2} / \mathrm{s} \pm 0.016$ s.e.m. $)$ and GalC $\left(0.32 \mu \mathrm{m}^{2} / \mathrm{s} \pm 0.026\right.$ s.e.m. $)$ in the PtK2 and fast in oligodendrocytes: $0.45 \mu \mathrm{m}^{2} / \mathrm{s} \pm 0.06$ s.e.m. for $\mathrm{SM}$ and $0.42 \mu \mathrm{m}^{2} / \mathrm{s} \pm 0.044$ s.e.m. for GalC.

Next, we divided values of diffusion coefficient, acquired at the highest STED power, by values of diffusion coefficient measured at confocal effective beam diameter $\left(\mathrm{D}_{\text {conf }}\right)(\mathrm{d}=250 \mathrm{~nm}) . \mathrm{D}_{S T E D} / \mathrm{D}_{\text {conf }}$ provide us with a value of D-ratio: representation of the level of anomality of the diffusion. The closer D-ratio is to 1 , the lower is anomality of the diffusing molecule and vice versa. Based on the experimental data (Mueller et al., 2011), we chose D-ratios below 0.5 to describe an anomalous diffusion.

As it is shown in Figure 3.3, PE and $\mathrm{SM}$ in oligodendrocytes have a tendency to the free diffusion mode, since their D-ratio approaches 1. GalC in oligodendrocytes shows a tendency to an anomalous diffusion, however not as prominent as in PtK2 cells. D-ratio in oligodendrocytes for GalC $-0.62 \mu \mathrm{m}^{2} / \mathrm{s} \pm 0.07$ s.e.m., for $\mathrm{SM}-0.86 \pm 0.13$ s.e.m., and for $\mathrm{PE}-0.73 \pm 0.09$ s.e.m. In the case of PtK2 cells, only D-ratio for the PE indicates free diffusion (D-ratio $=1.16 \pm 0.13$ s.e.m.), whereas D-ratios for SM and GalC are low $(0.44 \pm 0.06$ s.e.m. and $0.42 \pm 0.06$ s.e.m. , respectively) and show an anomalous diffusion. Since D-ratio for GalC in oligodendrocytes is very close to an anomalous diffusion threshold, we decided to further analyse the diffusion behaviour of tested lipids.

To further distinguish an anomalous diffusion from a free behaviour we used a method, proposed by Lenne and co-workers (Lenne et al., 2006). In this method, one measures diffusion coefficient at different STED powers, starting from $250 \mathrm{~nm}$ of the confocal effective beam diameter and down to the lowest STED effective beam diameter possible (usually the lowest effective beam diameter is setup-dependent). The values of the diffusion coefficient are then plotted as a function of the corresponding STED effective beam diameter $\left[\tau \mathrm{D}\left(d^{2}\right)\right]$. The acquired plot was named FCS diffusion law or FCS diffusion plot (Wawrezinieck et al., 2005). For each type of the anomalous diffusion the simulated slope fits are calculated. This simulations are used to fit experimental data. In brief, the expected $\tau \mathrm{D}\left(d^{2}\right)$ dependencies from the theoretical fit are as follows: $\tau \mathrm{D}$ is expected to vary linearly with $d^{2}$ for free diffusion; $\tau \mathrm{D}$ is a concave function of $d^{2}$ for transient trapping into nanodomains.

To build our experimental FCS diffusion plots, we measured diffusion coefficients at different STED powers, from $250 \mathrm{~nm}$ of confocal effective beam diameter to $40 \mathrm{~nm}$ of the last effective STED beam diameter, with several steps in between. Acquired FCS diffusion laws for the oligodendrocytes and PtK2 cells are shown in the Figure 3.4. Based on the proposed theoretical fits, PE, and SM in oligodendrocytes exhibit free diffusion without trapping, whereas only PE in PtK2 cells undergoes a free diffusion, with SM and GalC being strongly trapped (Mueller et al., 2011). GalC in oligodendrocytes has a slight trapping, however, not as strong as in PtK2 cells. 


\section{D-ratio of PE, SM and GalC in control PtK2 cells and oligodendrocytes}

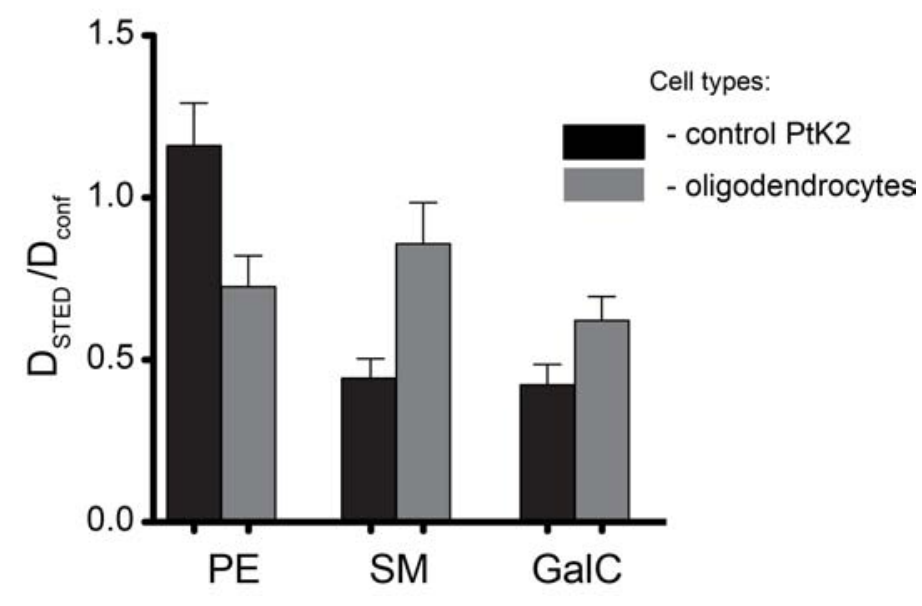

Figure 3.3: Diffusion ratio of PE, SM and GalC in oligos is higher than in PtK2 cells. Cultured oligodendrocytes on day 5 were incubated with a mixture of BSA-coupled artificial lipids labelled with Atto647N. After 30 minutes incubation with BSA-coupled lipids, oligodendrocytes were subjected to a STED-FCS. PtK2 control cells were subjected to the same procedure. Diffusion ratio, as a measure of the anomalous diffusion, is shown for PE, SM and GalC. D-ratio is acquired by dividing diffusion coefficient measured at the STED lowest effective beam diameter $(40 \mathrm{~nm})$ by diffusion coefficient measured at the confocal effective beam diameter $(250 \mathrm{~nm})$ : $\mathrm{D}_{S T E D} / \mathrm{D}_{\text {conf }}$. This value represents the anomality of the diffusion: the more D-ratio deviates from 1 , the more anomalous is the diffusion. D-ratio lower than 0.5 was considered to indicate trapping. $\mathrm{N}=20$ cells, error bars represent s.e.m. 

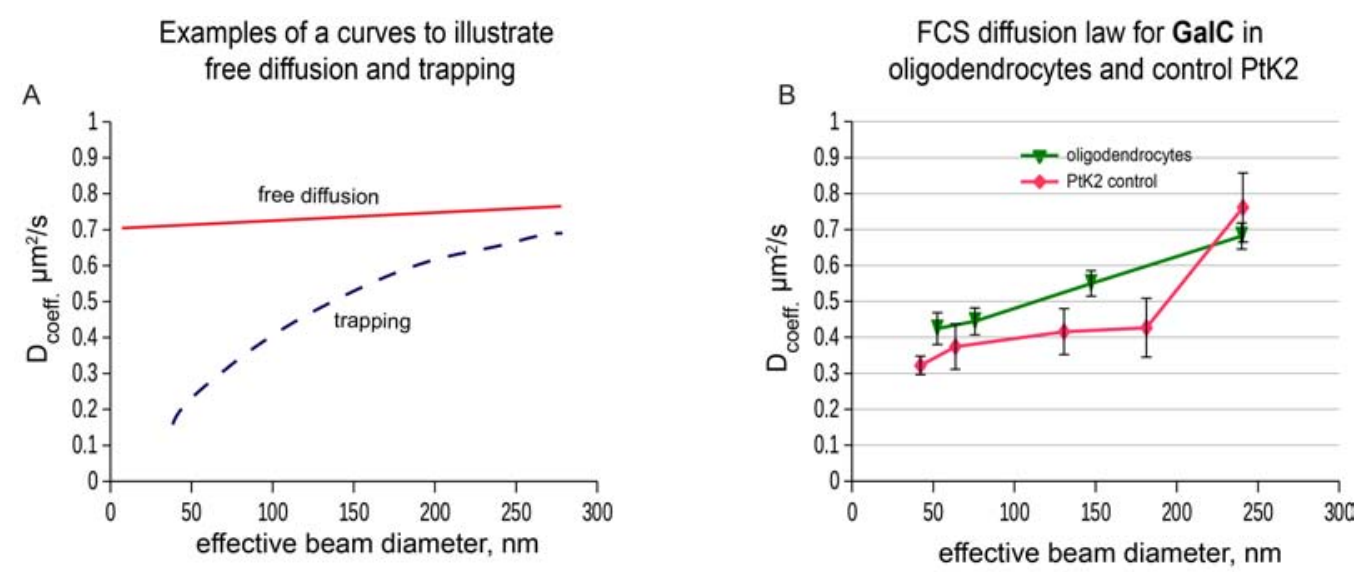

C

FCS diffusion law for PE in oligodendrocytes and control PtK2

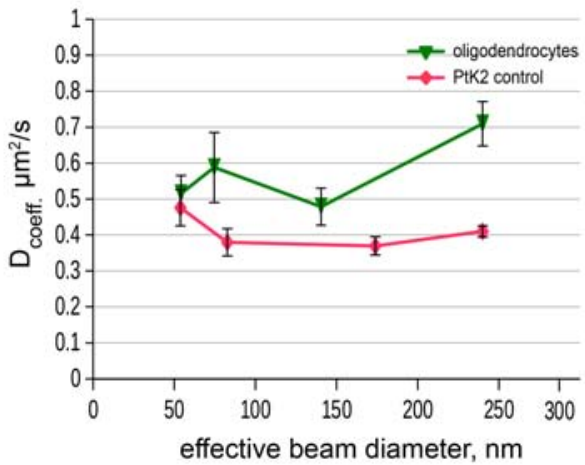

D

FCS diffusion law for SM in oligodendrocytes and control PtK2

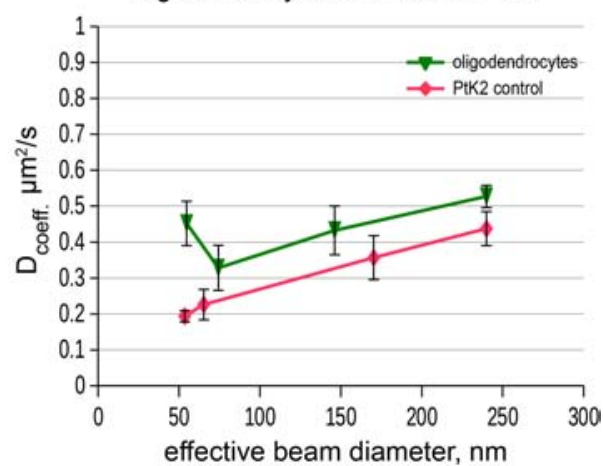

Figure 3.4: FCS diffusion plots for SM and PE in oligodendrocytes demonstrate free diffusion mode, and decrease of trapping for GalC. Cultured oligodendrocytes on day 5 were incubated with a mixture of BSA-coupled artificial lipids labelled with Atto647N. After 30 minutes incubation with BSA-coupled lipids, oligodendrocytes were subjected to a STED-FCS. PtK2 control cells were subjected to the same procedure. (A) Representative shapes of the curves for free diffusion and trapping are depicted. FCS diffusion plots for GalC (B), PE (C) and SM (D) in oligodendrocytes (green) and PtK2 (red) cells. $\mathrm{N}=20$ cells, error bars represent s.e.m. 


\subsection{Mimicking the oligodendrocyte sheets in PtK2 cells}

A cellular system to reconstitute myelin sheets in fibroblasts was recently developed (Aggarwal et al., 2013). In brief, a chimeric construct that contains MBP, transmembrane domain, GFP, and endoplasmatic reticulum (ER)-retention signal (MBP-GFP-TM) was designed (Figure 2.1 in Methods Section 2.4.4). PtK2 cells, transfected with this chimeric construct hereafter are referred to as PtK2-MBP + . Upon transfection of PtK2 fibroblast cell line, this construct gets anchored within the ER membrane, with GFP inside the ER lumen and MBP facing the plasmalemma. Here MBP interacts with the negatively charged lipids and brings two adjacent membranes (ER and cellular) together. The areas where MBP brings in contact two membranes are GFP-positive (Figure 3.5). In a number of experiments it was shown that these MBP + GFP-positive regions closely resemble oligodendrocyte sheets: filamentous actin is depleted in these regions, cytoplasm is squeezed out and cytoplasmic and transmembrane proteins are extruded (Aggarwal et al., 2013). Representative images of the transfected PtK2-MBP + cells are shown in Figure 3.5: middle panel (Figure 3.5 B1-B3) shows absence of actin in the MBP + GFP-positive regions and lower panel (Figure 3.5 C1-C3) depicts extrusion of glycoproteins.

We used this sheet-mimicking system as a convenient tool to answer the question whether MBP might influence lipid behaviour. We performed measurements of the lipid diffusion in the GFP-positive $\mathrm{MBP}+$ regions of PtK2 cells transfected with MBP-GFP-TM. Values of diffusion coefficient and D-ratios, acquired for PE, SM and GalC in PtK2-MBP + cells, compared to previous data from control (non-transfected) PtK2 cells and oligodendrocytes, are shown on Figure 3.6 and Figure 3.8.

Interestingly, diffusion coefficient at $40 \mathrm{~nm}$ effective STED beam diameter in the PtK2-MBP + for all three lipids was very high: $0.73 \mu \mathrm{m}^{2} / \mathrm{s} \pm 0.07$ s.e.m. for GalC, $0.73 \mu \mathrm{m}^{2} / \mathrm{s} \pm 0.08$ s.e.m. for $\mathrm{PE}$ and $0.75 \mu \mathrm{m}^{2} / \mathrm{s} \pm 0.0$ s.e.m. for $\mathrm{SM}$, in comparison to PtK2 control cell and oligodendrocytes (Figure 3.6).

Figures 3.7 and 3.8 show D-ratio and FCS diffusion laws for the lipids of interest in three cellular systems. As we can see from these data, mode of diffusion for PE remains the same. However its speed changes: within the PtK2-MBP + regions $\mathrm{PE}$ becomes faster than in oligodendrocyte sheets. Diffusion mode of the SM and GalC changes drastically: trapping disappears. Thus, in the regions where MBP brings two adjacent membranes together creating sheets-like structures, mode of lipid diffusion changes from trapping to free diffusion, together with an overall increase of the diffusion velocity. 


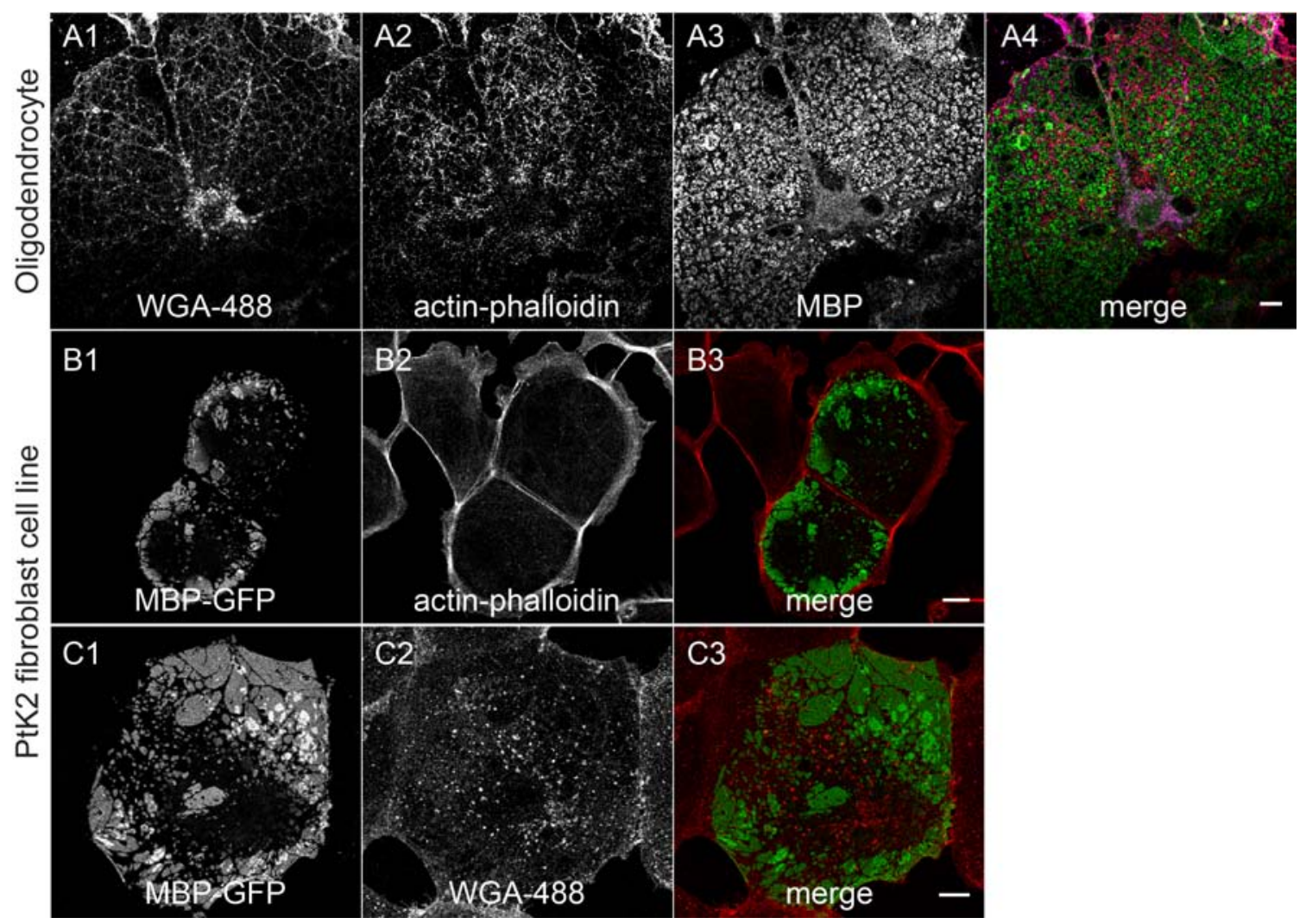

Figure 3.5: Oligodendrocyte sheets and sheets-like regions within PtK2-MBP + cells are free of actin and glycoproteins. Cultured oligodendrocytes were fixed with PFA 4\% + GTA $0.25 \%$ on day 5 and incubated with WGA-488 leptin (A1, cyan in A4), Rhodamine-phalloidin (A2, red in A4) and anti-MBP antibodies (A3, green in A4) to visualise glycoproteins, actin and compact membrane sheets, respectively. Cultured PtK2 cells were transfected with MBP-encoding construct $(\mathrm{PtK} 2-\mathrm{MBP}+)(\mathrm{B}-\mathrm{C})$ and fixed with PFA $4 \%+$ GTA $0.25 \% 16$ hours after transfection. MBP + regions were highlighted due to GFP expression (B1, C1, green in B3 and C3). After fixation cells were incubated with Rhodamine-phalloidin (B2, red in B3) and WGA-488 leptin (C2, red in C3), to visualise actin and glycoproteins, respectively. Glycoproteins and actin are absent in both, oligodendrocyte sheets and in the $\mathrm{MBP}+$ regions of the transfected PtK2-MBP + cells. Scale bar $=10 \mu \mathrm{m}$. 


\section{Diffusion coefficient for PE, SM and GalC in control PtK2 cells, oligodendrocytes and PtK2-MBP+}

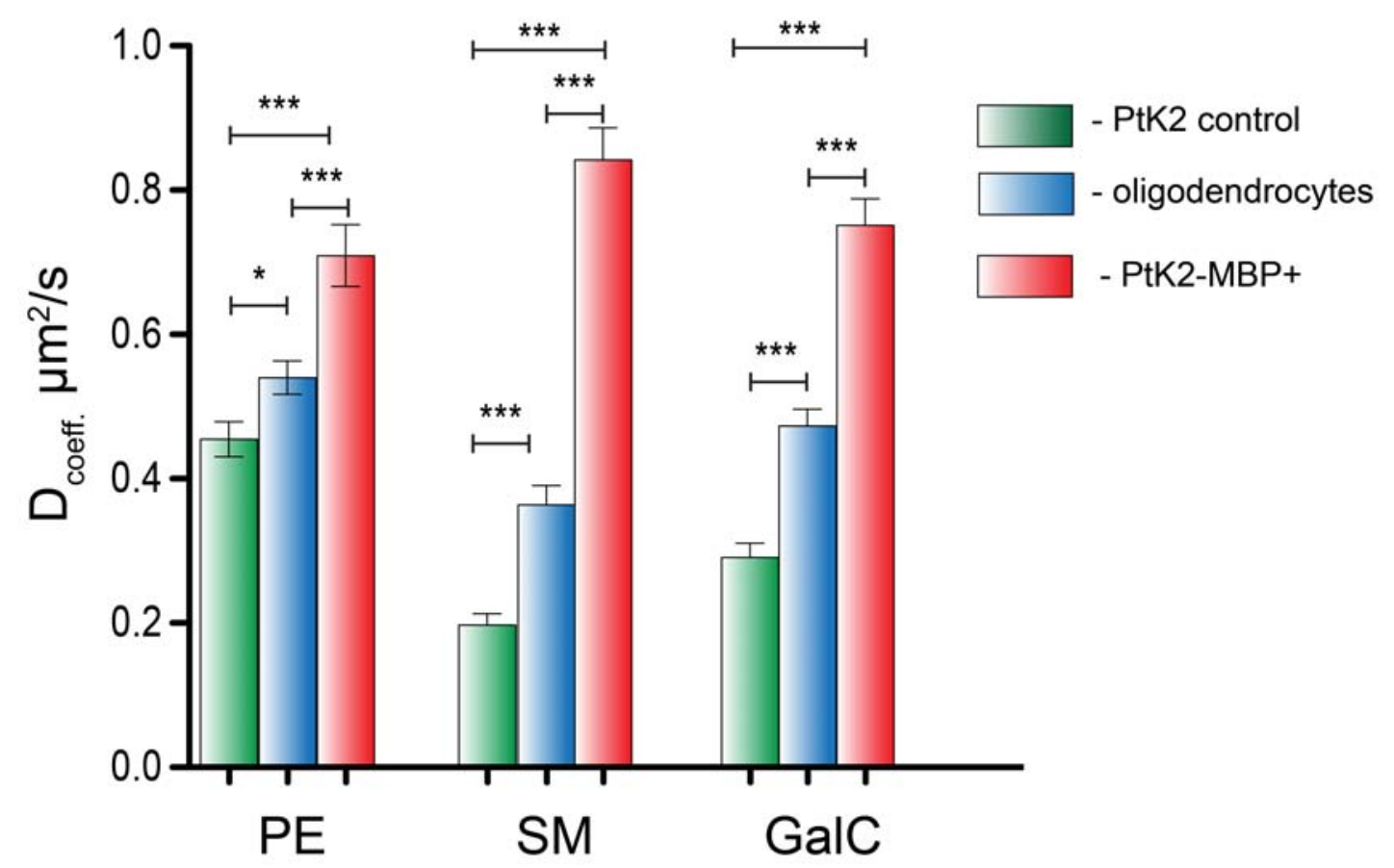

Figure 3.6: Diffusion coefficient of the $\mathrm{SM}$ and GalC in PtK2-MBP + regions is higher than in oligodendrocyte sheets and control PtK2 cells. Cultured oligodendrocytes on day 5 were incubated with a mixture of BSA-coupled artificial lipids labelled with Atto647N. After 30 minutes incubation with BSA-coupled lipids, oligodendrocytes were subjected to a STED-FCS. PtK2 control cells were subjected to the same procedure. Cultured PtK2 cells were transfected with MBP-encoding construct (PtK2-MBP + ) and lipid diffusion within MBP-positive regions was measured $18-20$ hours post transfection. $\mathrm{MBP}+$ regions within transfected cell were visualised due to GFP-expression. Artificial lipids were introduced into the membrane of transfected cells via BSA-coupled method, see Section 2.7.6 in Methods. PE, SM and GalC diffusion coefficients were acquired at the highest STED power $(\mathrm{d}=40 \mathrm{~nm})$ for PtK2 (green), oligodendrocytes (blue), and $\mathrm{PtK} 2-\mathrm{MBP}+$ (red) cells. Diffusion coefficient for PE is very similar in all cellular systems. GalC and SM diffusion is faster in oligodendrocytes, than in PtK2 cells and even faster in PtK2-MBP+ regions, compared to the oligodendrocyte sheets. $\mathrm{N}=20$ cells, error bars represent s.e.m.; ${ }^{*}-\mathrm{p}<$ $0.05, * * *-\mathrm{p}<0.0001$, t-test. 


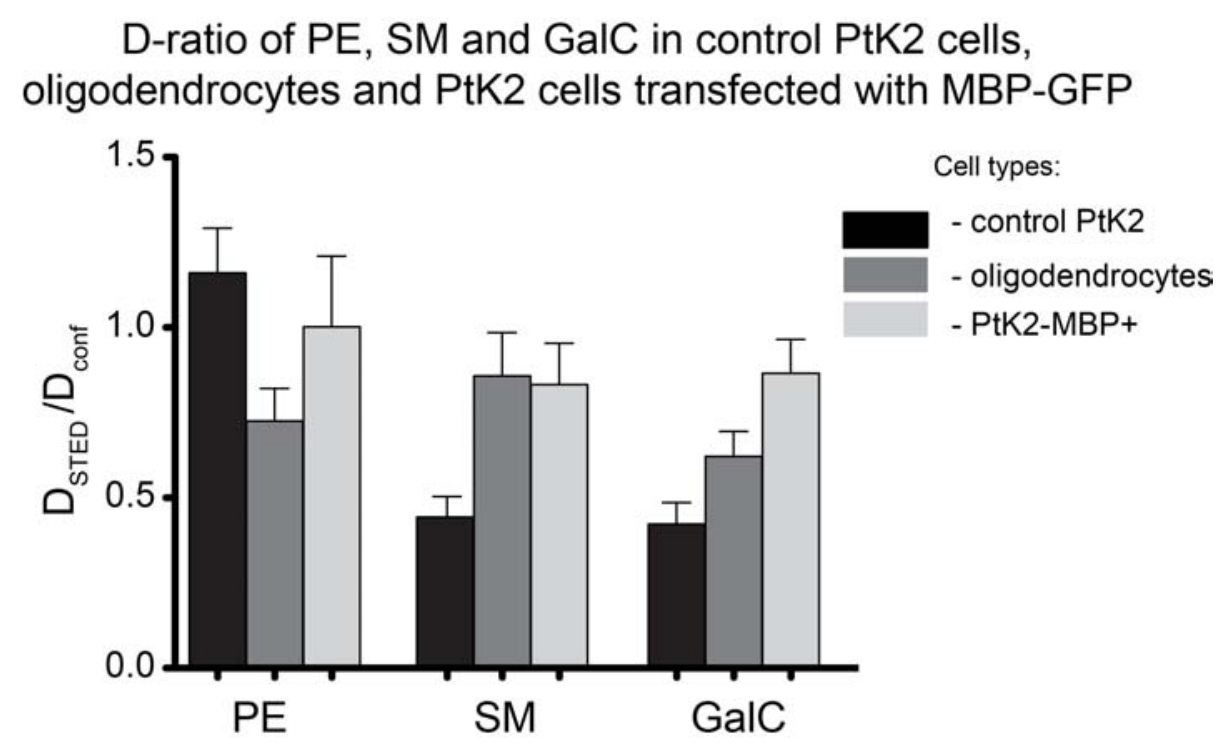

Figure 3.7: Diffusion ratio of the PE, SM and GalC in PtK2-MBP + regions is higher than in oligodendrocyte sheets and control PtK2 cells. Cultured oligodendrocytes on day 5 were incubated with a mixture of BSA-coupled artificial lipids labelled with Atto647N. After 30 minutes incubation with BSA-coupled lipids, oligodendrocytes were subjected to a STED-FCS. PtK2 control cells were subjected to the same procedure. Cultured PtK2 cells were transfected with MBP-encoding construct $(\mathrm{PtK} 2-\mathrm{MBP}+$ ) and lipid diffusion within $\mathrm{MBP}+\mathrm{GFP}$-positive regions was measured $18-20$ hours post transfection. $\mathrm{MBP}+$ regions within transfected cell were visualised due to GFP-expression. Artificial lipids were introduced into the membrane of transfected cells via BSA-coupled method, see Section 2.7.6 in Methods. Diffusion ratio, as a measure of the anomalous diffusion, is shown for PE, SM and GalC. D-ratio is acquired by dividing diffusion coefficient at the STED lowest effective beam diameter $(40 \mathrm{~nm})$ by diffusion coefficient at the confocal effective beam diameter $(250 \mathrm{~nm}): \mathrm{D}_{S T E D} / \mathrm{D}_{\text {conf }}$. This value represents the anomality of the diffusion: the more D-ratio deviates from 1 , the more anomalous is the diffusion. D-ratio lower than 0.5 was considered to indicate trapping. $\mathrm{N}=20$ cells, error bars represent s.e.m. 
A FCS diffusion law for GalC in oligodendrocytes and control PtK2

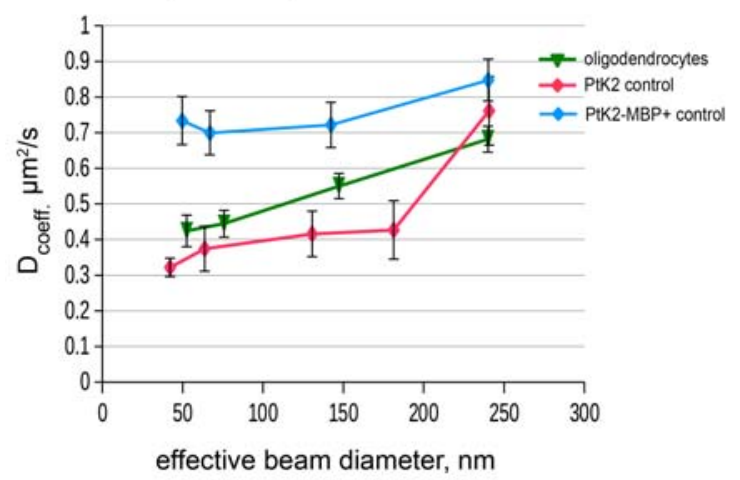

B

FCS diffusion law for PE in oligodendrocytes and control PtK2

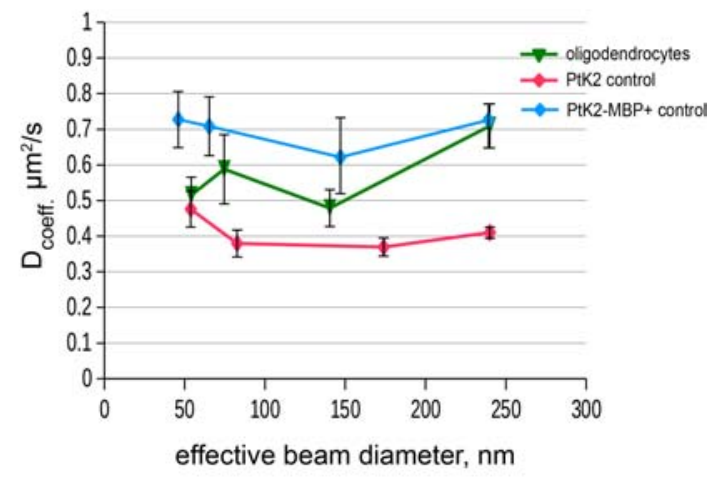

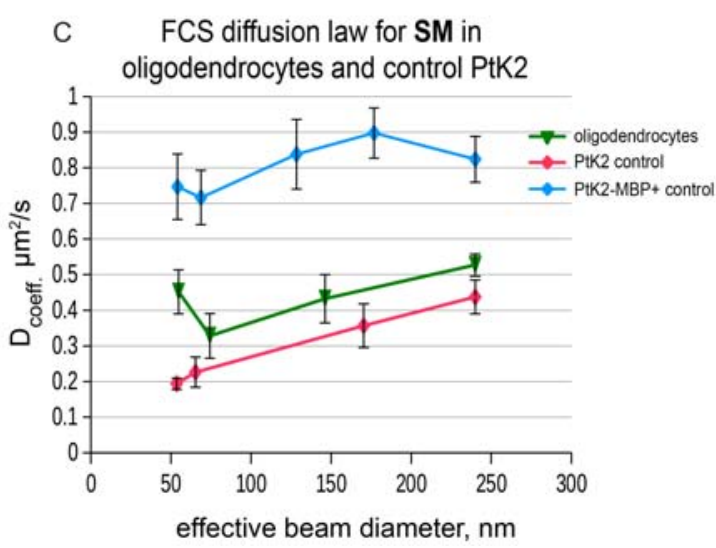

Figure 3.8: FCS diffusion plots for SM, PE and GalC in PtK2-MBP + regions demonstrate absence of the anomalous diffusion. Cultured oligodendrocytes on day 5 were incubated with a mixture of BSA-coupled artificial lipids labelled with Atto647N. After 30 minutes incubation with BSA-coupled lipids, oligodendrocytes were subjected to a STED-FCS. PtK2 control cells were subjected to the same procedure. Cultured PtK2 cells were transfected with MBP-encoding construct $(\mathrm{PtK} 2-\mathrm{MBP}+)$ and lipid diffusion within $\mathrm{MBP}+\mathrm{GFP}$-positive regions was measured 18 -20 hours post transfection. $\mathrm{MBP}+$ regions within transfected cell were visualised due to GFPexpression. Artificial lipids were introduced into the membrane of transfected cells via BSA-coupled method, see Section 2.7.6 in Methods. FCS diffusion plots for GalC (A), PE (B) and SM (C) in oligodendrocytes (green), PtK2 (red) and PtK2-MBP + (blue) cells are shown. According to the plots, all three lipids tested exhibit a free diffusion within MBP + GFP-positive regions of transfected PtK2, when compared to control PtK2. Moreover, lipids within MBP + domains have a faster diffusion, when compared to oligodendrocytes and control PtK2 cells. $\mathrm{N}=20$ cells, error bars represent s.e.m. 


\subsection{Effects of fatty acid chain length}

All lipids that are described in this study contain short-chain (C16) fatty acids. As was mentioned before, myelin sheaths are enriched in long-chain fatty acids lipids (C20, C23, C24) that influence the thickness of the myelin membrane. Length of the fatty acid chain might have an impact on diffusion properties. Therefore, we used an additional artificial lipid - Thr-Cerebroside-KK114 - as a long-chain analog of the endogenous galactosylcerebroside (GalC), further referred to as 23C-GalC. This lipid was labelled with KK114 dye and not with Atto647N. Data was collected only for the control PtK2 cells and transfected PtK2-MBP + (Figure 3.9). We did not observe any difference between both cell systems. In both systems $23 \mathrm{C}-\mathrm{GalC}$ was extremely slow: in control cells the diffusion coefficient was $0.29 \mu \mathrm{m}^{2} / \mathrm{s} \pm 0.03$ s.e.m. and in MBP-PtK2 $+-0.25 \mu \mathrm{m}^{2} / \mathrm{s} \pm 0.025$ s.e.m. Intriguingly, in both systems, 23C-GalC had a free mode of diffusion (Figure 3.9 B and C). Since the 23C-GalC lipid is labelled with another dye, a possible impact of the labelling should be taken into account when comparing the data to the short-chain GalC. So far, we base our conclusions only on the measurements acquired from the short-chain lipids.

Based on the data obtained so far, we suggest an absence of constrained diffusion in oligodendrocyte sheets for the short-chain lipids. MBP might contribute to the membrane lipid rearrangements to facilitate this uniform lipid distribution, via extruding proteins and, most importantly actin, from underneath the membrane. This results in the release of membrane lipids from the constrains for free diffusion the proteins usually create. Thus, short-chain lipids, regardless of their nature (phospholipids, cerebroside or sphingomyelin), get homogeneously distributed within oligodendrocyte sheet. 
A Diffusion coefficient of $23 \mathrm{C}-\mathrm{GalC}$ in control PtK2 and in PtK2-MBP+

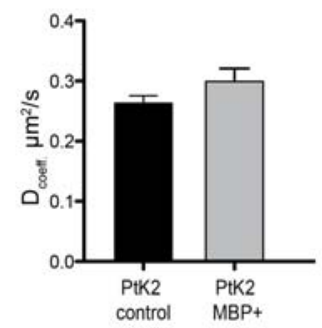

B D-ratio of $23 \mathrm{C}-\mathrm{GalC}$ in control PtK2 and in PtK2-MBP+

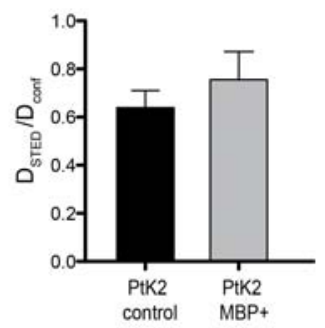

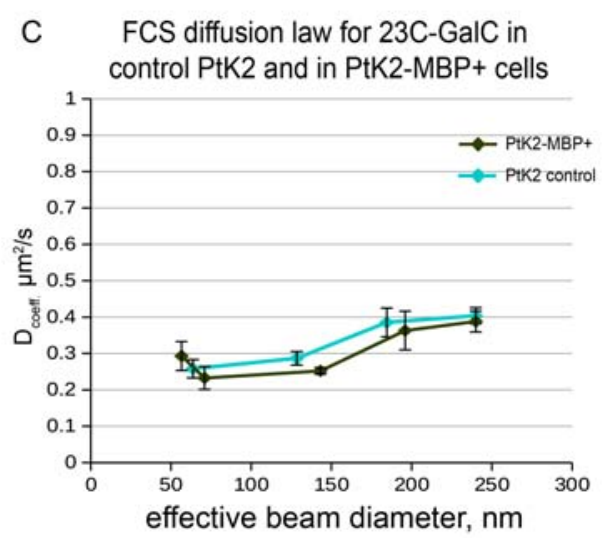

Figure 3.9: Diffusion characteristics for 23C-GalC in PtK2 control and PtK2-MBP+ cells. Cultured control PtK2 cells were incubated with a mixture of BSA-coupled 23C-GalC. After 30 minutes incubation PtK2 cells were subjected to a STED-FCS. Cultured control PtK2 cells were transfected with MBP-encoding construct (PtK2-MBP + ) and lipid diffusion within MBP + GFPpositive regions was measured $18-20$ hours post transfection. $\mathrm{MBP}+$ regions within transfected cell were visualised due to GFP-expression. 23C-GalC was introduced into the membrane of transfected cells via BSA-coupled method, see Section 2.7.6 in Methods. Diffusion characteristics for 23C-GalC in PtK2 control and PtK2-MBP + cells are shown in the graphs. (A) Diffusion coefficient acquired at the highest STED power for PtK2 control (black) and PtK2-MBP + (grey). N $=5$ cells. (B) Diffusion ratio, as a measure of the anomalous diffusion, is shown for 23C-GalC. D-ratio is acquired by dividing diffusion coefficient measured at the STED lowest effective beam diameter $(40 \mathrm{~nm})$ by diffusion coefficient measured at the confocal effective beam diameter $(250 \mathrm{~nm}): \mathrm{D}_{S T E D} / \mathrm{D}_{\text {conf }}$. This value represents the anomality of the diffusion: the more D-ratio deviates from 1, the more anomalous is the diffusion. D-ratio lower than 0.5 was considered to indicate trapping. D-ratio for 23C-GalC suggests a free diffusion in both cellular systems for this lipid. (C) FCS diffusion law of 23C-GalC for PtK2 control (light blue) and PtK2-MBP + (green). According to the plots, 23C-GalC exhibits a free diffusion in the both cellular systems. $\mathrm{N}=3$ cells. 


\section{Part II: Polarity of the phosphatidylinositols in the oligodendrocytes}

\subsection{Distribution of phosphatidylinositols in oligoden- drocytes}

Under the common term cellular polarity one describes the asymmetric organisation of the cell, including cellular surface components, intracellular organelles distribution and cytoskeleton rearrangements. Polarity is a ubiquitous feature among all species in all classes of eucariotes, from the unicellular yeast, to the multicellular organisms. Cellular polarity of the plasma membrane is an important regulator, involved in all crucial organismal life points, from the global events of cellular migration, tissue formation and differentiation during the development, to the more minor, such as epithelium function maintenance, blood cell migration and cellular division (Mellman \& Nelson, 2008).

There are several common mechanistic themes, simple variation of which results in the perfect orchestration of the polarity events (for more information, see Section 1.6).

Polarity plays an important role in the development of CNS and PNS. Polarisation of axons occurs very early in the development, when the growth cone of a neuron starts to extend towards the gradient, established by growth factors. This extension is characterised by accumulation of $\mathrm{PIP}_{3}$ in the growing tip, followed by axon polarisation and further outgrowth. Oligodendrocytes, the myelin producing cell in the CNS, are also polarised and form specific domains, both in vivo and in vitro (Figure 1.9 and Figure 3.1).

This part of my $\mathrm{PhD}$ thesis was aimed to explore the polarised distribution of phosphatidylinositols in the oligodendrocytes, using 2D cultures of the mouse oligodendrocytes, as a model system.

\subsubsection{Defining the differential localisation of $\mathrm{PIP}_{2}$ and $\mathrm{PIP}_{3}$ in the mature oligodendrocytes}

In this Section, the morphological characterisation of the phosphatidylinositols localisation within the membrane of cultured oligodendrocytes is described. Analysis was performed using immunocytochemistry, associated with laser scanning confocal microscopy.

First, immunocytochemical analysis with anti-PIP ${ }_{2}$ and anti-PIP $\mathrm{P}_{3}$ antibodies revealed very poor detection of these phosphatidylinositols within all parts of oligodendrocytes (Figure 3.10 A-B). It was reported that phosphatidylinositols are extremely sensitive to the fixative agents due to their easy extractability form the membrane 
during fixation. In our case, usage of only $4 \%$ PFA led to washing out most of the phosphatidylinositols, leading to dramatic signal decrease. However, mixing 4\% PFA with $0.25 \%$ GTA (Sharma et al., 2008) resulted in a significant increase of the signal quality and overall signal-to-noise ratio (Figure 3.10 C-D). All further immunolabeling protocols were done, using $4 \%$ PFA with $0.25 \%$ GTA as a fixatives.

Immunocytochemical analysis, with a proper fixating conditions revealed similar localisation of phosphatidylinositols in the mature oligodendrocytes: both were mostly enriched in the non-compact domains (Figure 3.11).

Due to its highly positive charge, MBP interacts directly with negatively charged phospholipids of the inner leaflet (Musse et al., 2008; Harauz et al., 2009; Nawaz et al., 2009). We suggested that such interaction might occur as well between MBP and phosphatidylinositols in the inner leaflet of the myelin membrane in the compact region. This interaction might have influenced the immunolabelling, by masking the phosphatidylinositol epitopes from the antibodies. To elucidate this we detached MBP from the membrane with subsequent labelling of $\mathrm{PIP}_{2}$. MBP detachment upon Ionomycin treatment of the cells is an established method by Nawaz (2009). Indeed, after 2 min of Ionomycin treatment, we could detect an increase of the $\mathrm{PIP}_{2}$ signal within the sheets (Figure $3.12 \mathrm{~A}-\mathrm{B}$ ). Intriguingly, we haven't observed any changes within $\mathrm{PIP}_{3}$ localisation upon Ionomycin treatment (Figure 3.12 C-D).

To support our findings, we used an alternative to conventional antibodies labelling: small molecular probes, possessing $\mathrm{PH}$-domains that specifically recognise either $\mathrm{PIP}_{2}$ (mCitrine-PLC $\gamma$ ) or $\mathrm{PIP}_{3}$ (mCherry-Grp1), (Figure 3.13) (van den Bogaart et al., 2011). Cells were treated with Ionomycin, as described previously. Again, consistent with the antibody labelling, upon detachment of MBP from the membrane we could identify $\mathrm{PIP}_{2}$ (Figure $3.13 \mathrm{~A}-\mathrm{B}$ ), but not $\mathrm{PIP}_{3}$ (Figure $3.13 \mathrm{C}-\mathrm{D}$ ) in the sheet region. Quantifications of the signal intensity distribution from processes to sheets upon Ionomycin treatment shows that the $\mathrm{PIP}_{2}$ signal in sheets remains on the same level, as in processes, whereas $\mathrm{PIP}_{3}$ signal drops (Figure $3.13 \mathrm{E}$ and $\mathrm{F}$ ). 
PFA 4\%

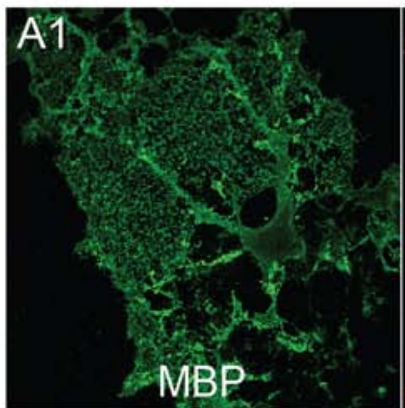

A2

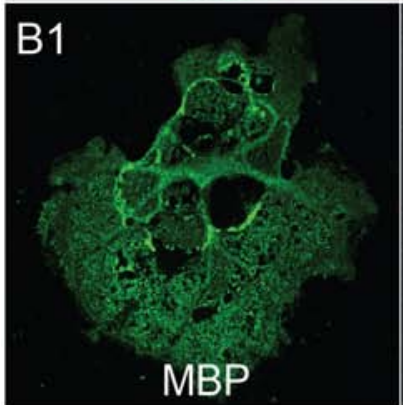

B2

PIP2
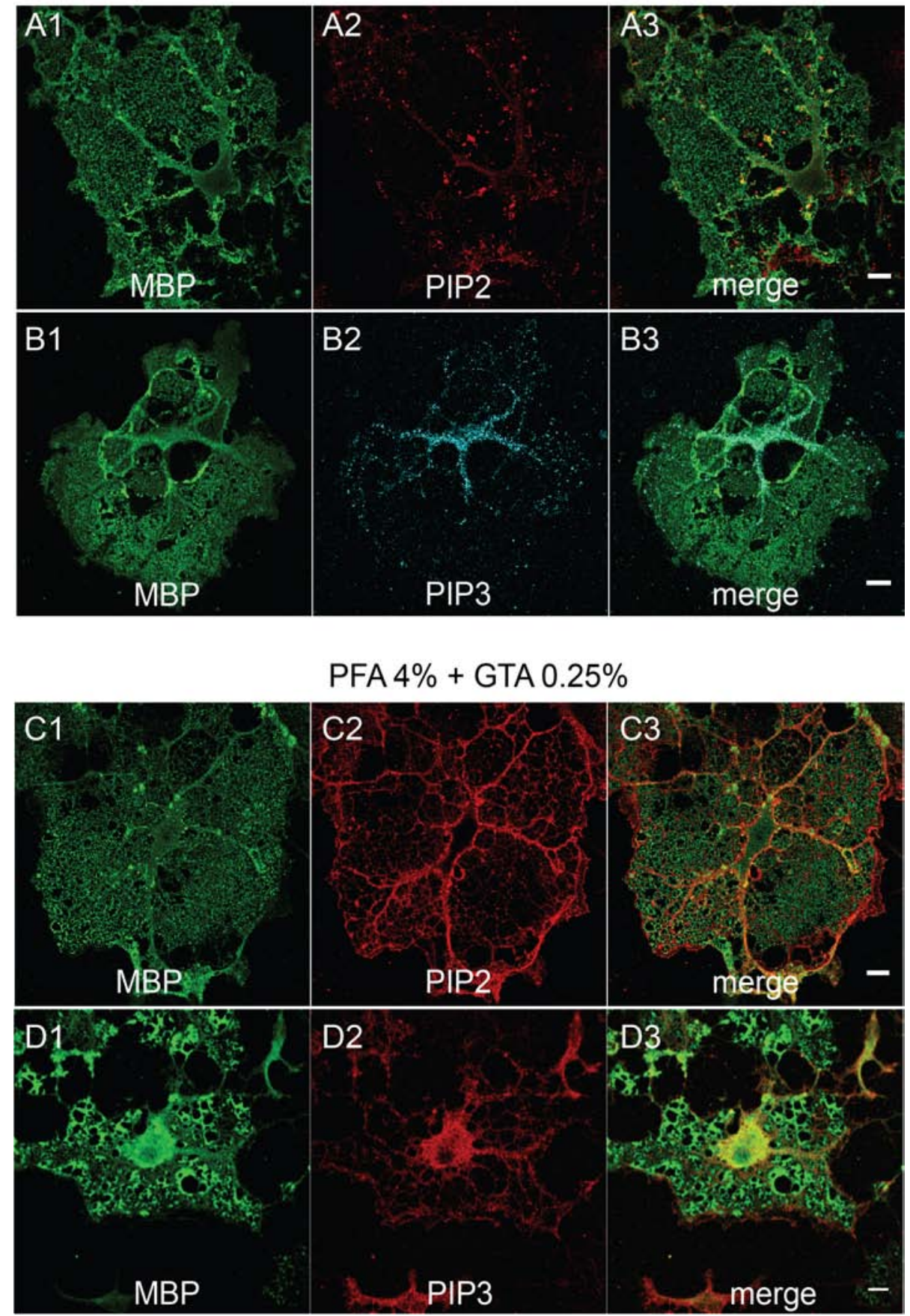

Figure 3.10: Fixative agents extract $\mathrm{PIP}_{2}$ and $\mathrm{PIP}_{3}$, decreasing efficiency of antibody labelling. Cultured oligodendrocytes were fixed with $4 \%$ paraformaldehyde (PFA 4\%) (A-B) or with $4 \%$ paraformaldehyde, combined with $0.25 \%$ glutaraldehyde (PFA 4\% + GTA 0.25\%) (C-D) on day 5. After fixation cells were treated with anti-MBP antibodies (in green - A1, B1, C1, $\mathrm{D} 1)$ to visualise membrane sheets, and either with anti-PIP $\mathrm{P}_{2}$ (red - A2, C2) or anti-PIP 3 (cyan - B2, red - D2) antibodies to visualise localisation of phosphatidylinositols. Phospholipids are easy to extract from the cellular membrane, hence the appropriate fixative agent is of a great importance. Enrichment of $4 \%$ paraformaldehyde fixative with $0.25 \%$ of glutaraldehyde increased fixation reaction speed and thus both $\mathrm{PIP}_{2}$ and $\mathrm{PIP}_{3}$ are better preserved within the membrane (C-D), in comparison to $4 \%$ paraformaldehyde alone (A-B). Scale bar $=10 \mu \mathrm{m}$. 


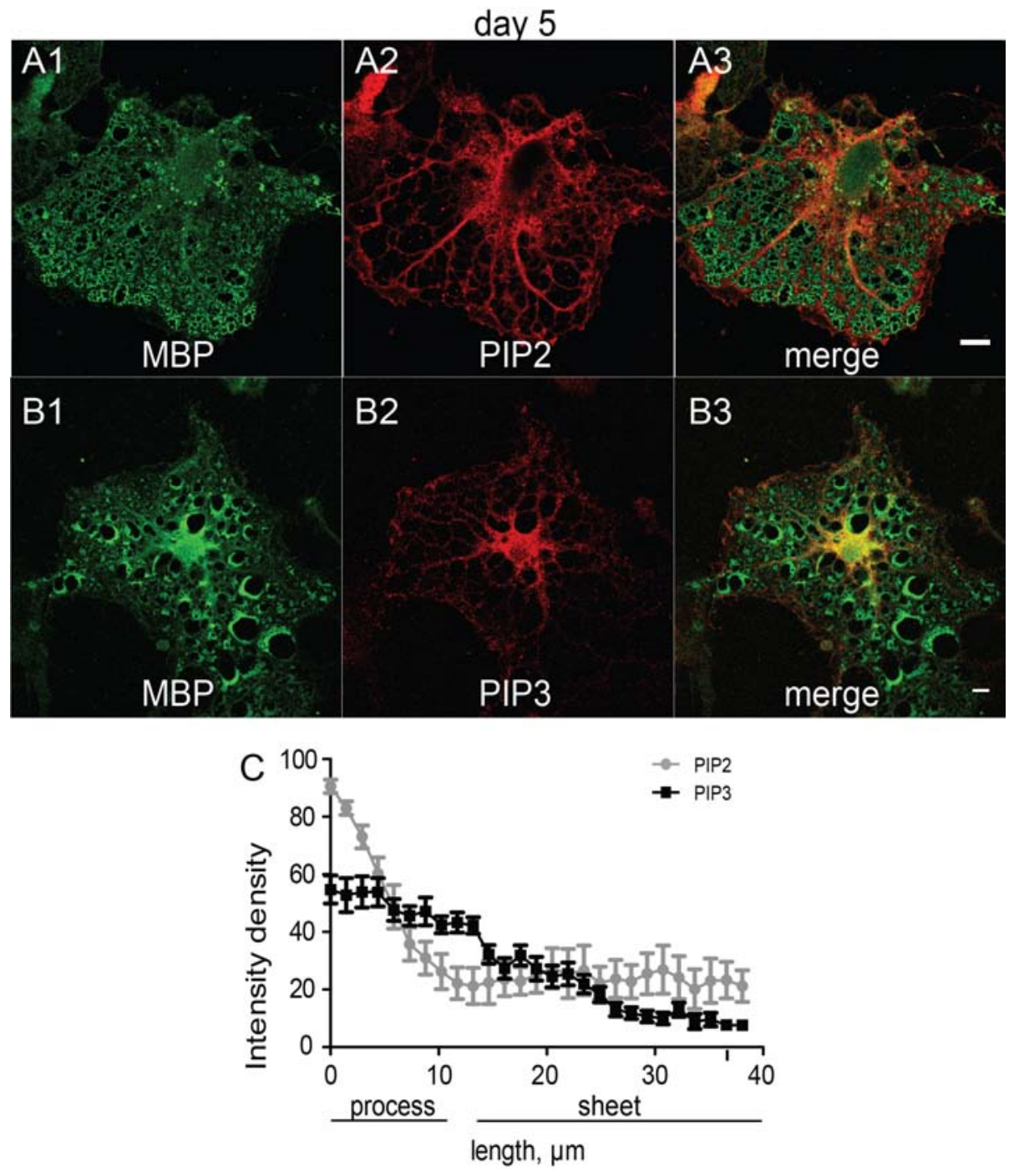

Figure 3.11: $\mathrm{PIP}_{2}$ and $\mathrm{PIP}_{3}$ are found in the processed and in the rim of mature oligodendrocytes. Cultured oligodendrocytes were fixed with PFA $4 \%+$ GTA $0.25 \%$ on day 5 . After fixation, cells were treated with anti-MBP antibodies (green - A1 and B1) to visualise sheets and either with anti-PIP 2 (red - A2) or anti-PIP 3 (red - B2) antibodies to visualise localisation of phosphatidylinositols. Distribution of the signal intensity (intensity density) for $\mathrm{PIP}_{2}$ and $\mathrm{PIP}_{3}$ is shown as a line profile plot (C). Antibody signal for $\mathrm{PIP}_{2}$ and $\mathrm{PIP}_{3}$ was measured from a middle of process as a starting point and towards the sheet as an end point $(40 \mu \mathrm{m}$ of the total length measured). Signal distribution between $\mathrm{PIP}_{2}$ (grey) and $\mathrm{PIP}_{3}$ (black) varies only slightly. Scale bar $=10 \mu \mathrm{m}$. Error bars represent s.e.m. $\mathrm{N}=3$, with at least 20 cells per experiment. 

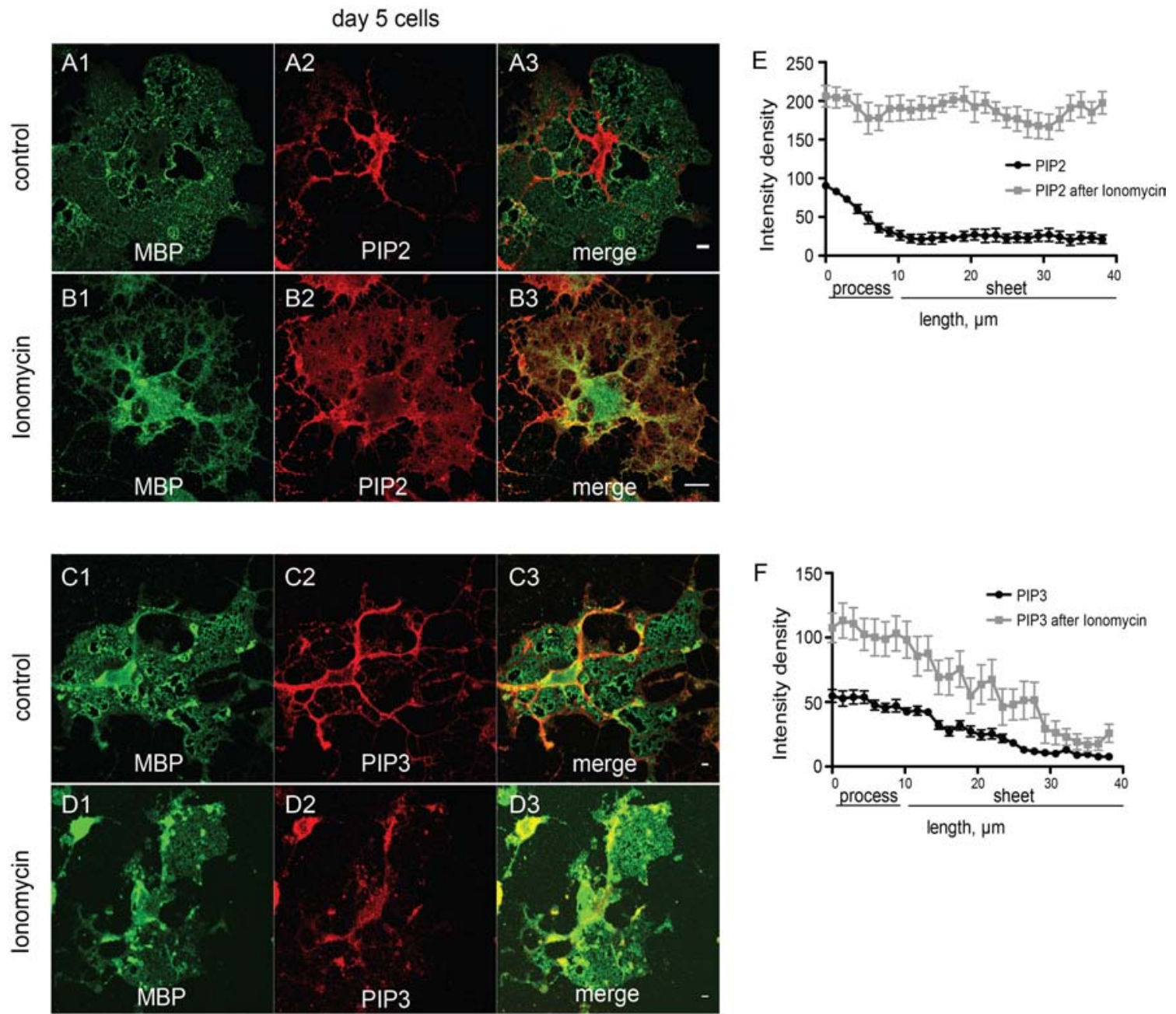

Figure 3.12: $\mathbf{P I P}_{2}$, but not $\mathrm{PIP}_{3}$ is masked by MBP in the sheets of oligodendrocytes. Cultured oligodendrocytes on day 5 were treated with $10 \mu \mathrm{M}$ Ionomycin to detach MBP from the membrane (Nawaz et al., 2009) and subsequently fixed with PFA $4 \%+$ GTA $0.25 \%$. After fixation, cells were treated with anti-MBP antibodies (green - A1-D1) to visualise sheets and either with anti-PIP 2 (red - A2, B2) or anti-PIP 3 (red - C2, D2) antibodies to visualise localisation of phosphatidylinositols. Upon the shift of intracellular membrane charge, MBP detached from the membrane with subsequent $\mathrm{PIP}_{2}$ exposure (unmasking). This resulted in the increase of anti-PIP $\mathrm{P}_{2}$ antibody signal in the sheet region (B2). Interestingly, Ionomycin treatment of oligodendrocytes did not lead to an increase of $\mathrm{PIP}_{3}$ signal in the sheets and $\mathrm{PIP}_{3}$ remained enriched strictly at the margins and processes zones (D2). Line profile plot of the signal intensity densities of $\mathrm{PIP}_{2}$ (E, black squares - control, grey squares - after treatment) and $\mathrm{PIP}_{3}$ (F, black squares - control, grey squares - after treatment) demonstrates the effect of Ionomycin treatment on the $\mathrm{PIP}_{3}$ distribution. Antibody signal for $\mathrm{PIP}_{2}$ and $\mathrm{PIP}_{3}$ was measured from a middle of a process as a starting point and towards the sheet as an end point (40 $\mu \mathrm{m}$ of the total length measured). $\mathrm{PIP}_{2}$ signal increases drastically, upon its unmasking from MBP, with $\mathrm{PIP}_{3}$ showing the same tendency as in the non-treated condition. Scale bar $=10 \mu \mathrm{m}$. Error bars represent s.e.m. $\mathrm{N}=3$, with at least 20 cells per experiment. 
day 5 cells
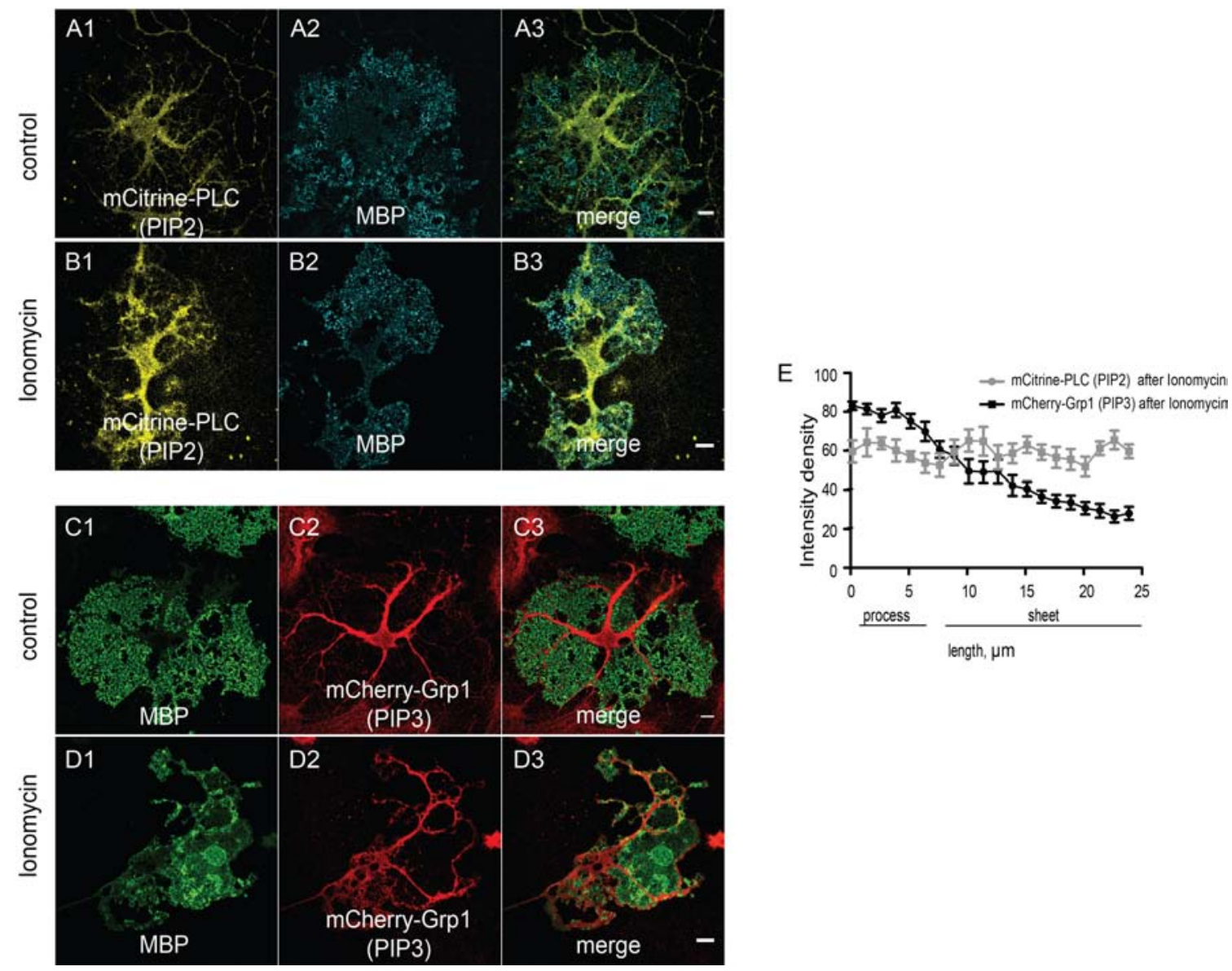

Figure 3.13: $\mathrm{PIP}_{3}$ localises strictly to the processes and the rim in oligodendrocytes. Cultured oligodendrocytes on day 5 were treated with $10 \mu \mathrm{M}$ Ionomycin to detach MBP from the membrane (Nawaz et al., 2009) and subsequently fixed with PFA $4 \%+$ GTA $0.25 \%$. After fixation, cells were treated with small fluorescent probes to visualise $\mathrm{PIP}_{2}$ (mCitrine-PLC, yellow - A1, B1) or $\mathrm{PIP}_{3}$ (mCherry-Grp1, red - C2, D2) and with anti-MBP antibody (cyan - A2, $\mathrm{B} 2$ and green - C1, D1) to visualise sheets. Upon the shift of intracellular membrane charge, MBP detached from the membrane with subsequent $\mathrm{PIP}_{2}$ exposure (unmasking). This resulted in the increase of anti-PIP $\mathrm{PP}_{2}$ antibody signal in the sheet region (B1). Ionomycin treatment of oligodendrocytes did not lead to an increase of $\mathrm{PIP}_{3}$ signal in the sheets and $\mathrm{PIP}_{3}$ remained enriched strictly at the margins and processes zones (D2). (E) Line profile plot of the signal intensity densities of $\mathrm{PIP}_{2}$ (grey squares) and $\mathrm{PIP}_{3}$ (black squares) demonstrates the effect of Ionomycin treatment on the phosphatidylinositols distribution. Antibody signal for $\mathrm{PIP}_{2}$ and $\mathrm{PIP}_{3}$ was measured from a middle of a process as a starting point and towards the sheet as an end point $(25 \mu \mathrm{m}$ of the total length measured). As in the case of immunocytochemistry, upon Ionomycin treatment, $\mathrm{PIP}_{2}$ gets visible in sheets whereas enrichment of $\mathrm{PIP}_{3}$ remains strictly at the cell margins and processes zones. Scale bar $=10 \mu \mathrm{m}$. Error bars represent s.e.m. $\mathrm{N}=3$, with at least 20 cells per experiment. 


\subsubsection{Defining the onset of the separate localisation of phosphati- dylinositols}

Next, we addressed the question at what point of the oligodendrocytes development this segregation of phosphatidylinositols occurs. Thus, we labelled phosphatidylinositols in the OPC (day 1 cells in culture) to visualised their localisation on the early developmental stage. Intriguingly, both phospholipids showed rather uniform distribution over the sheet region (Figure 3.14), with $\mathrm{PIP}_{3}$ levels being slightly higher at the rim and in the processes (Figure 3.14 B2). We could conclude, thus, that $\mathrm{PIP}_{2}$ and $\mathrm{PIP}_{3}$ are first uniformly distributed and their segregation occurs only upon cell maturation.

To further support our findings, we decided to transfect oligodendrocytes with plasmids including specific PH-domains, fused to GFP: PLC-GFP for $\mathrm{PIP}_{2}$ recognition and Akt-GFP for $\mathrm{PIP}_{3}$ recognition. Transfection of oligodendrocytes was done at two different developmental stages: matured cells - day 4 and immature cells day 1, with subsequent staining either at day 5 (Figure $3.15 \mathrm{C}-\mathrm{D}$ ) or at day 2 (Figure 3.15 A-B), after 16-18 hours post-transfection.

At the early stage, $\mathrm{PIP}_{2}$ and $\mathrm{PIP}_{3}$ were uniformly distributed over the sheet region. Upon cell maturation, signal from both phosphatidylinositols became visible only in the rim and processes. This cellular localisation of $\mathrm{PIP}_{3}$ is in line with the observation from the antibody labelling. Residing of $\mathrm{PIP}_{2}$ in the non-compact rim and processes at the mature stage was expected, due to the fact that MBP does not permit bulky proteins (as PLC-GFP is) to enter the sheet region at this stage (Aggarwal et al., 2011).

\subsubsection{Role of MBP in the localisation of phosphatidylinositols}

The major change that distinguish between immature oligodendrocytes from mature ones is a presence of MBP-zipped sheets. Hence, based on the knowledge that MBP was reported to interact with negatively charged lipids, we though that this protein might be involved in the active segregation of two different phosphatidylinositols' species into separate domains.

To test this assumption, we used an MBP-free model system: oligodendrocytes derived from the shiverer mutant mice. These cells still are able to develop sheets, however these structures remain unzipped, due to the lack of MBP expression. We again performed antibody staining (Figure 3.16) to address the question, whether the presence of MBP is crucial for the phosphatidylinositols differential location.

Intriguingly, shiverer oligodendrocytes did not exhibit phosphatidylinositols separation on the mature stage: both, $\mathrm{PIP}_{2}$ and $\mathrm{PIP}_{3}$ are distributed throughout sheets and processes (Figure 3.16). Therefore, these experiments supported our idea that for phosphatidylinositols to be separated into different domains MBP presence is crucial.

\subsubsection{MBP interactions with differently charged phosphati- dylinositols}

It was shown previously that MBP interaction with phosphatidylinositols is of an electrostatic nature (Musse et al., 2008; Harauz et al., 2009; Nawaz et al., 2009). 

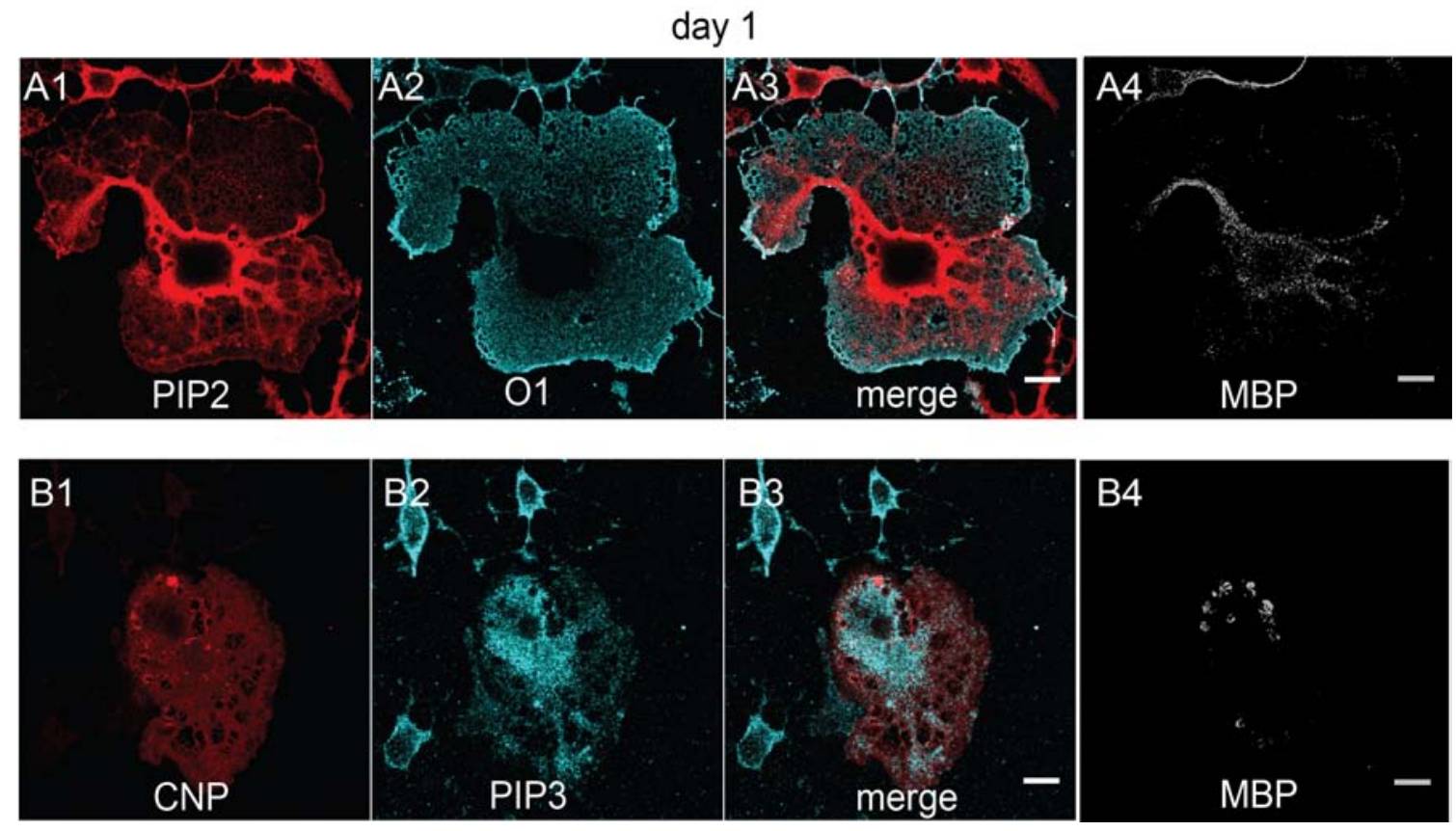

Figure 3.14: $\mathrm{PIP}_{2}$ and $\mathrm{PIP}_{3}$ localise to the sheets at the early stages of oligodendrocytes. Cultured oligodendrocytes on day 1 were fixed with PFA $4 \%$ + GTA $0.25 \%$. After fixation, cells were treated with anti-CNP (red - B1) and anti-O1 (cyan - A2) to visualise yet non-compact sheet regions of the immature cell, with anti-MBP antibody (grey - A4, B4) to ensure that cells reside in the immature stage and with anti-PIP $\mathrm{PI}_{2}$ (red - A1) and anti-PIP 3 (cyan - B2) to visualise phosphatidylinositols localisation at the early stage. Antibody labelling at this stage revealed that $\mathrm{PIP}_{2}$ starts to fill up the sheet before MBP translation takes place. $\mathrm{PIP}_{2}$ is more uniformly distributed throughout the sheet $(\mathrm{A} 1)$, than $\mathrm{PIP}_{3}$, with latter one being more pronounce in the process region and on the rim (B2). Scale bar $=10 \mu \mathrm{m}$.

Albeit, from results acquired so far, we observed that MBP masked only $\mathrm{PIP}_{2}$, but not $\mathrm{PIP}_{3}$. Therefore, we asked the question, whether MBP interacts with $\mathrm{PIP}_{3}$. To answer this question, we performed a liposomal pull-down of MBP. In this assay, phosphatidylcholine was chosen to be a liposome "body". PIP 2 was used as a positive control; phosphatidylserine and $\mathrm{PIP}_{3}$ - as examples of lipids with one and three negative charges, respectively. Non-charged phosphatidylethanolamine was used as a control. MBP was added to the each fraction of the liposomes in the non-saturating conditions. After incubation time, samples were centrifuged (see Methods Section 2.7.4 for details). Acquired pellet and supernatant were separately applied onto SDSPAGE gel and stained with Coomassie after gel run to determine in what fraction MBP amount was higher (Figure $3.17 \mathrm{~A}$ ). We expected MBP to precipitate upon binding with negatively charged liposomes and therefore to be enriched in the pellet fractions (Figure $3.17 \mathrm{~B}$ ). According to the liposomal pull-down, MBP indeed did not exhibit a preferential binding to any of the negatively-charged lipids tested. 

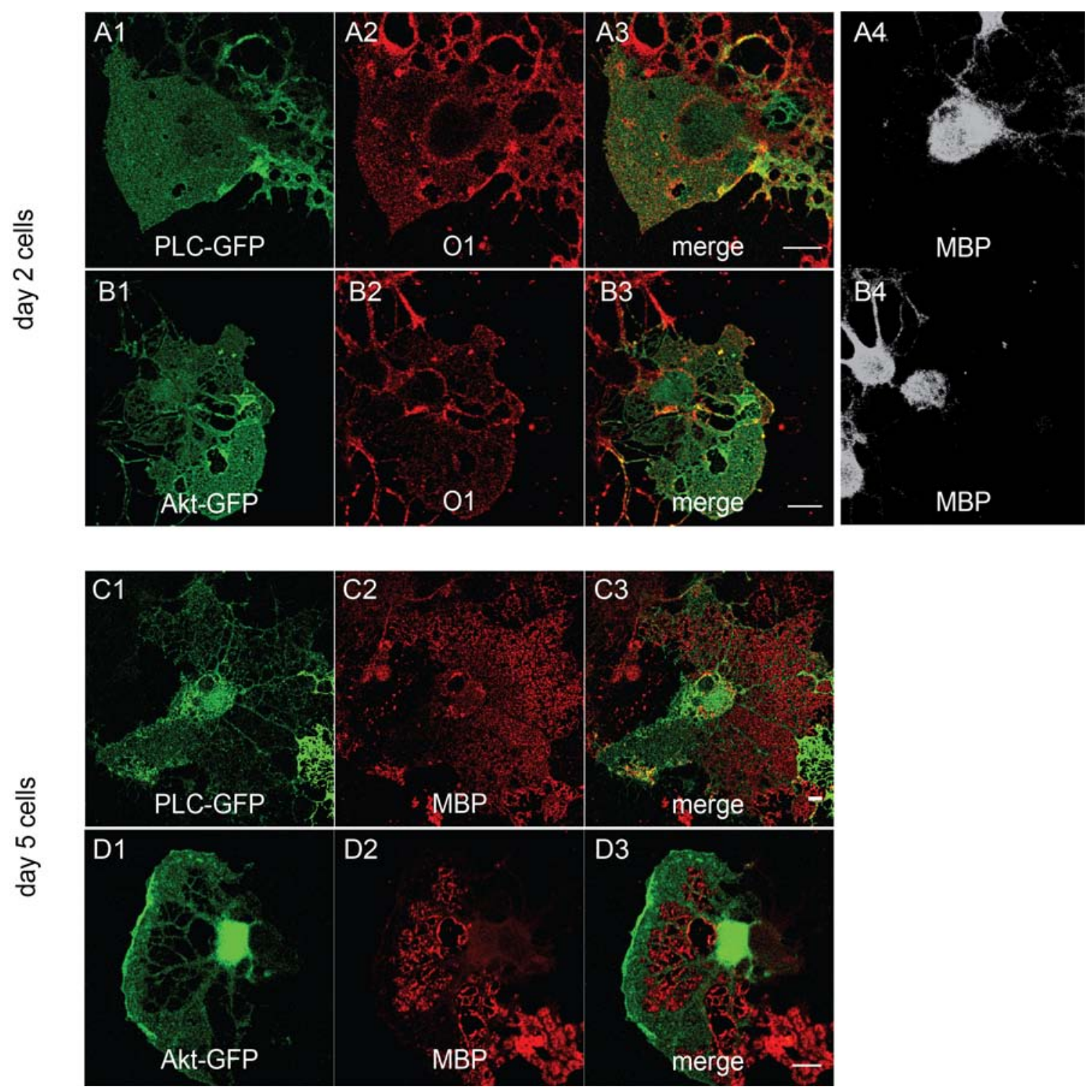

Figure 3.15: $\mathrm{PIP}_{3}$ localisation changes from uniformly distributed to processes and rimenriched upon oligodendrocytes maturation. Cultured oligodendrocytes were transfected

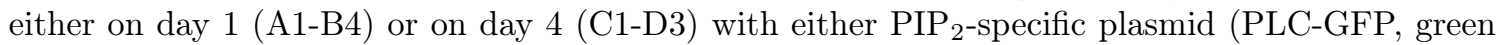

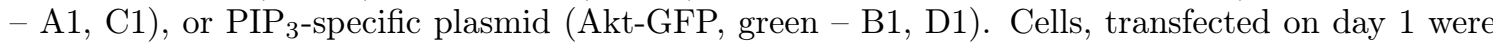
fixed with PFA $4 \%+$ GTA $0.25 \%$ after $18-20$ hours post-transfection (on day 2 ) and treated with anti-O1 antibody to visualise non-compacted sheets on day 2 (red - A2, B2) and with anti-MBP antibody (grey - A4, B4) to ensure absence of MBP expression. Cells, transfected on day 4 were fixed with PFA $4 \%+$ GTA $0.25 \%$ after 18 - 20 hours post-transfection (on day 5) and treated with anti-MBP antibody (red-C2, D2) to visualise compact sheets. When cells were transfected on day $1, \mathrm{PIP}_{2^{-}}$and $\mathrm{PIP}_{3}$-specific constructs were uniformly spread over whole cellular membrane after 18 hours post-transfection (A1 and B1). When cells were transfected on day 4, $\mathrm{PIP}_{2^{-}}$and $\mathrm{PIP}_{3}$-specific constructs were both residing in the processes and cellular margins after 18 hours post-transfection $(\mathrm{C} 1$ and $\mathrm{D} 1)$. This cellular localisation of $\mathrm{PIP}_{3}$ on day 5 is in line with the observation from the antibody labelling. Residing of $\mathrm{PIP}_{2}$-specific construct in the non-compact rim and processes at the mature stage was expected, due to the fact that MBP does not permit bulky proteins (as PLC-GFP is) to enter the sheet region at this stage (Aggarwal et al., 2011). Scale bar $=10 \mu \mathrm{m}$. 

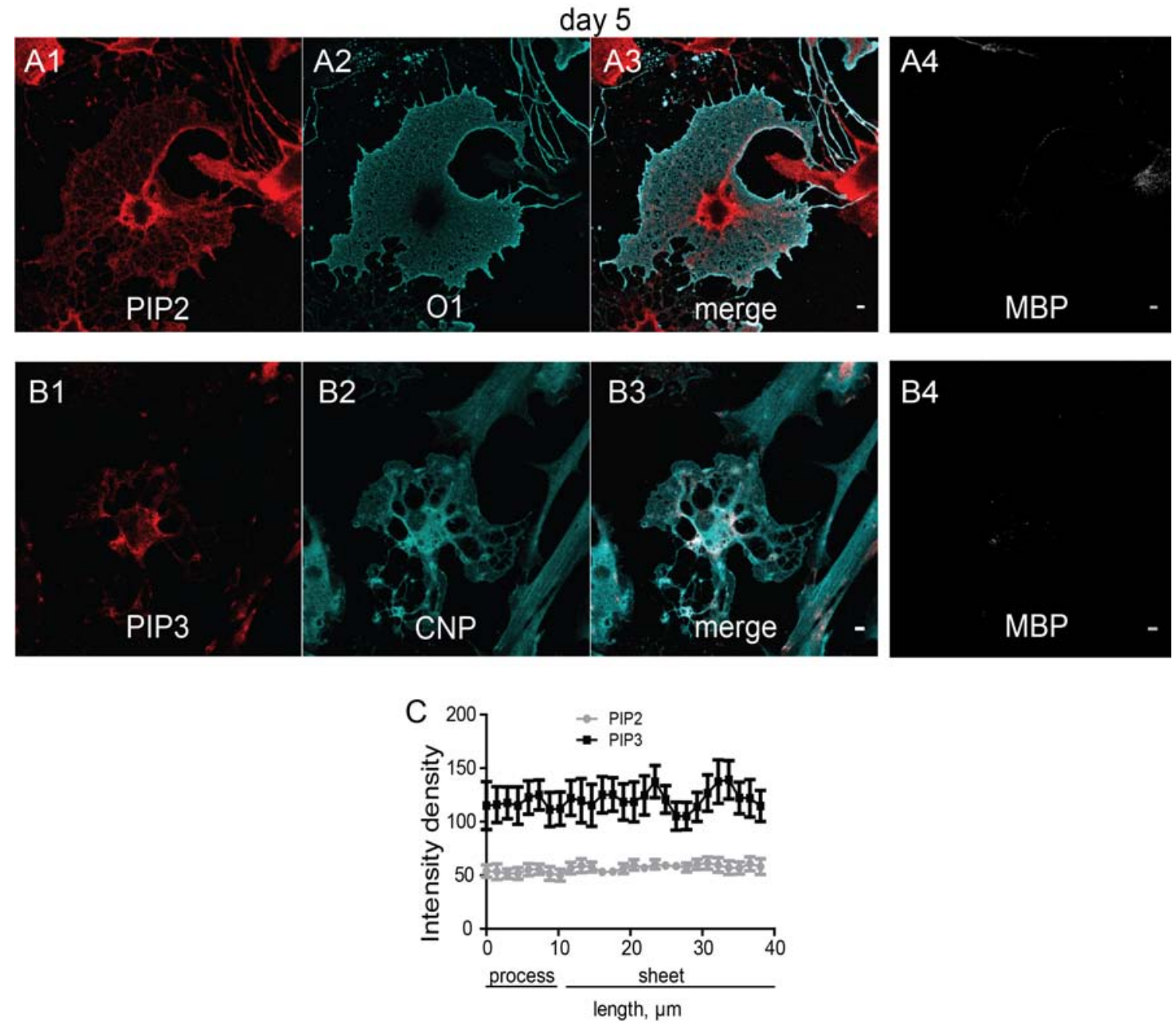

Figure 3.16: PIP $_{2}$ and PIP $_{3}$ are uniformly distributed in the membrane of oligodendrocytes from shiverer mice. Cultured oligodendrocytes from shiverer mice were fixed with PFA $4 \%+$ GTA $0.25 \%$ on day 5 and treated with anti-O1 (cyan - B1) and anti-CNP (cyan - B2) to visualise non-compact sheets of the mutant cell and with anti-MBP antibody (grey - A4, B4) to ensure that cells was shiverer and did not express MBP. Anti-PIP 2 (red - A1) and anti-PIP 3 (red - B1) antibodies were used to visualise localisation of phosphatidylinositols. Line profile plot of the signal intensity densities of $\mathrm{PIP}_{2}$ (C, grey squares) and $\mathrm{PIP}_{3}$ (C, black squares) demonstrated equal distribution of $\mathrm{PIP}_{2}$ and $\mathrm{PIP}_{3}$, disregarding membrane region (compare to Figure 3.11). Antibody signal for $\mathrm{PIP}_{2}$ and $\mathrm{PIP}_{3}$ was measured from a middle of a process as a starting point and towards the sheet as an end point ( $40 \mu \mathrm{m}$ of the total length measured). Scale bar $=10 \mu \mathrm{m}$. Error bars represent s.e.m. $\mathrm{N}=3$, with at least 10 cells per experiment. 


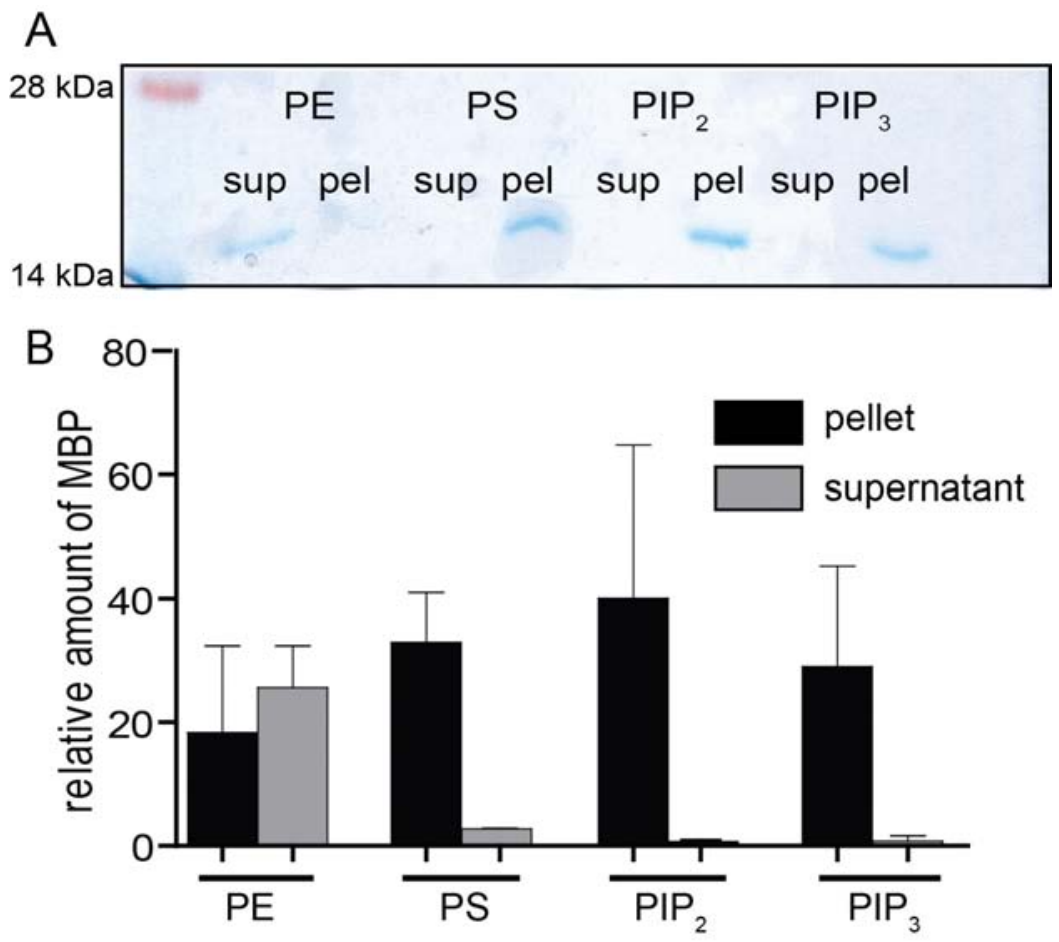

Figure 3.17: Pull-down of MBP by liposomes with different negatively charged lipids composition did not reveal preference of MBP binding to any of the phosphatidylinositols. Liposomes, composed of phosphatidylcholine as a liposomal 'body' and different negatively charged lipids $\left(\mathrm{PIP}_{2}\right.$ as a positive control; $\mathrm{PIP}_{3}$ and phosphatidylserine (PS) as triple- an singlecharged carriers) were prepared in a 80:20 ratio. Non-charged phosphatidylethanolamine (PE) was used as a control. After incubation time, samples were centrifuged (see Methods Section 2.7.4 for details). Acquired pellet and supernatant were separately applied onto SDS-PAGE gel and stained with Coomassie after gel run to determine in what fraction MBP amount was higher (A, sup supernatant, pel - pellet). Relative amount of MBP in each fraction was calculated, based on the relative densities of the Coomassie-stained bands (B). Liposomes with non-charged PE (control) did not pull-down MBP efficiently. MBP from the pellet in this case might come from its spontaneous oligomerisation and aggregation. Liposomes with phosphatidylserine (PS), $\mathrm{PIP}_{2}$ and $\mathrm{PIP}_{3}$ could precipitate $\mathrm{MBP}$ into the pellet, completely removing it from the supernatant. This data revealed no preferences of MBP binding to a particular kind of negatively-charged phospholipids tested. N $=3$, error bars represent SD. 


\section{7 $\mathrm{PIP}_{3}$ influences non-compact region of the oligo- dendrocytes}

Establishment of polarity plays an immense role in many processes in our organism, both in development and in adolescence. Brief overview of the factors, involved in the cellular polarity regulation, including phosphatidylinositols, is provided in Section 1.6 of Introduction. As it has been already mentioned, oligodendrocytes are morphologically polarised. This work has also demonstrated the existence of the phosphatidylinositol ' polarity in cultured oligodendrocytes. Our next question was: what role does polarity of phosphatidylinositols play in oligodendrocyte development.

\subsubsection{Interference with the endogenous $\mathrm{PIP}_{3}$ levels in the oligo- dendrocytes}

\section{Approach 1: increase of the $\mathrm{PIP}_{3}$ amount via inhibition of PTEN}

One of the major pathways that regulates levels of $\mathrm{PIP}_{3}$ and $\mathrm{PIP}_{2}$ in the distinct compartments of the cell is the mutual interconversion of these lipids by two enzymes: PI3K and PTEN. Phosphorylation by kinases (PI3K) produces $\mathrm{PIP}_{3}$ out of $\mathrm{PIP}_{2}$, and dephosphorylation of $\mathrm{PIP}_{3}$ by PTEN leads to generation of $\mathrm{PIP}_{2}$. Despite the fact that PTEN dephosphorylation of $\mathrm{PIP}_{3}$ is not the only pathway of the $\mathrm{PIP}_{3}$ turnover executed within the cells, it is considered to be a the major one (Sasaki et al., 2009).

To interfere with endogenous $\mathrm{PIP}_{3}$ and $\mathrm{PIP}_{2}$ levels, we chose to use VO-Ohpic inhibitor (further referred to as $\mathrm{VOOH}$ ) due to its high specificity and low toxicity at a low dosage (Tanaka et al., 2010; Katso et al., 2001).

Due to the former observation that separation of phosphatidylinositols occurs after the MBP translation (between day 2 and day 3 in culture), we applied PTEN inhibitor at the early developmental stage, namely on day 1 ( $24 \mathrm{hrs}$ after shake), with subsequent additional application on day 2 ( $48 \mathrm{hrs}$ after shake). Cells were grown till day 3 in culture, fixed and immunolabelled for compact and non-compact regions (MBP and CNP respectively). Additionally, cells were cultivated till mature stage day 5 - and fixed at this time point.

As a control for the successful PTEN inhibition we checked for the differences in the $\mathrm{PIP}_{3}$ amounts (Figure 3.18). As it was expected, PTEN inhibition with VOOH resulted in increased levels of endogenous $\mathrm{PIP}_{3}$.

Since PTEN is a known inhibitor for the cellular growth and proliferation, next question we would like to address was whether oligodendrocytes would have a faster rate of the sheet development upon PTEN inhibition. Hence, we subjected oligodendrocytes to the PTEN inhibitor treatment 16 hrs after shake, left cells to grow for another 12 hrs and then fixed and stained them for membrane sheet marker, CNP. The read out of this experiment was the amount of the oligodendrocytes that already on day 2 have developed CNP-positive and yet MBP-negative sheets. Figure 3.19 demonstrates that upon PTEN inhibition, oligodendrocytes tend to develop their sheets more rapidly than under normal circumstances.

To further describe morphological changes, size of the growth zone (outer rim region) and non-compact regions were quantified (Figure 3.20). In this experiment, cells were harvested either on their immature stage (day 3 ), or were allowed to reach 


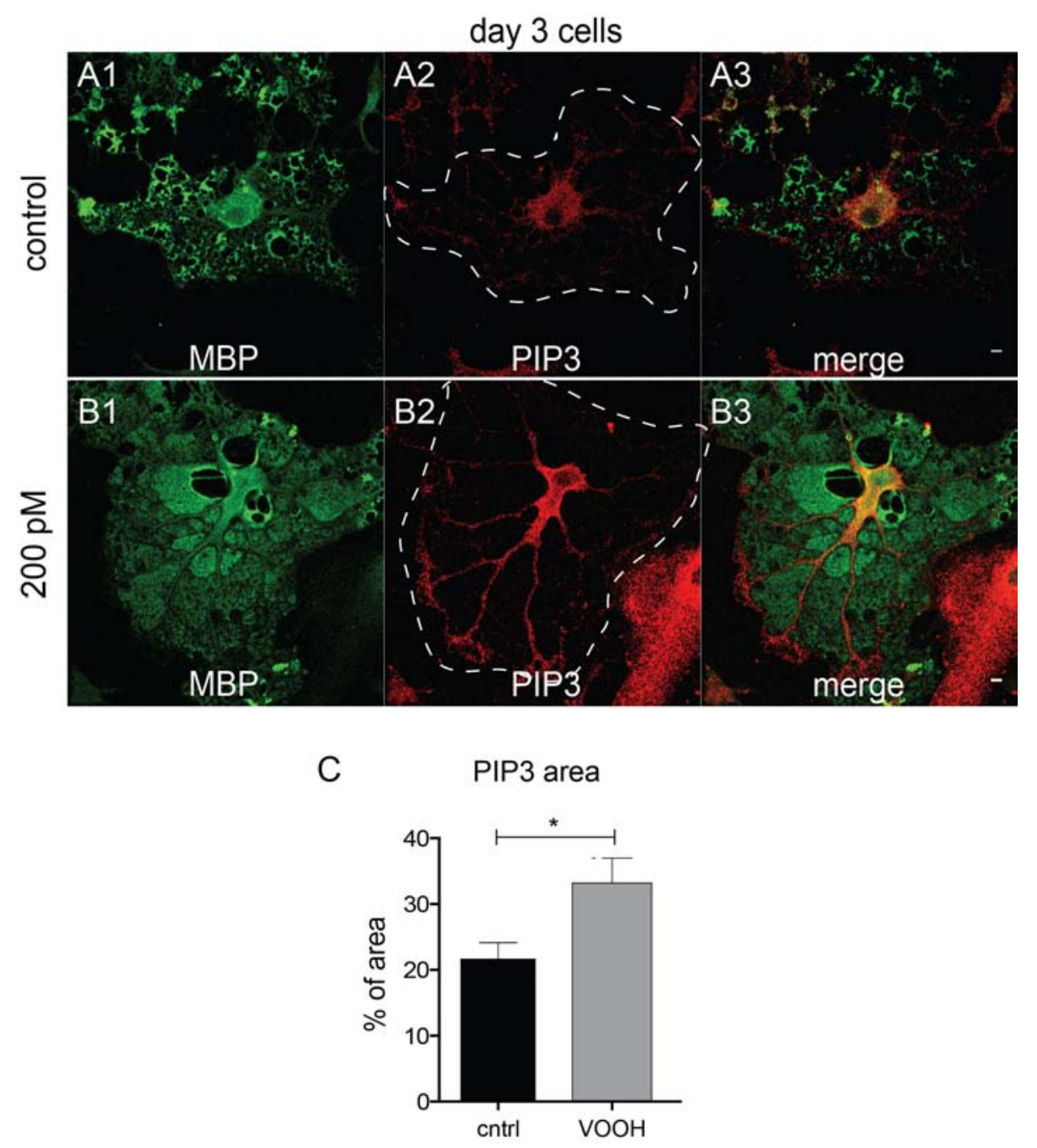

Figure 3.18: Treatment with 200 pM PTEN inhibitor leads to the increased amount of endogenous $\mathbf{P I P}_{3}$ in oligodendrocytes on day 3. Cultured oligodendrocytes were fixed with PFA $4 \%+$ GTA $0.25 \%$ on day 3. After fixation cells were treated with anti-MBP antibodies (green - A1, B1) to visualise membrane sheets, and with anti-PIP 3 (red - A2, B2). White dashed lines in A2 and B2 highlight the edges of the cells. Scale bar $=10 \mu \mathrm{m}$. (C) Quantification of the cell area, occupied by $\mathrm{PIP}_{3}$ in $\%$ of the total cell area, with (VOOH) and without (cntrl) PTEN inhibition. Mean \pm s.e.m. , $\mathrm{N}=2$, at least 15 cells per experiment, ${ }^{*}=\mathrm{p}<0.05$, t-test.

maturation (day 5). VOOH was not applied to the cells between day 3 and day 5 . To check whether normal cell morphology could be restored in the post-mature phases of the development, a population of cells was placed in the fresh medium without VOOH after maturation and they were kept in culture for the additional two days (till day 7) (Figure $3.20 \mathrm{~B}$ ). The growth zone size showed a significant increase only on day 5. Size of the growth zone was again restored after inhibition removal (Figure 3.20 B). When VOOH was not removed on day 5, cellular morphology was not restored (data not shown). Increase of the non-compact area after $\mathrm{VOOH}$ treatment was observed at immature and mature stages. Moreover, even at the very late stage of development (day 7 and 10), oligodendrocytes after $\mathrm{VOOH}$ treatment had increased size of the non-compact regions (Figure $3.20 \mathrm{C}$ ).

Since the growth zone was increased upon treatment of cells with VOOH, we also quantified amount of phosphoAkt. Phosphorylated Akt is an active form of Akt, one 


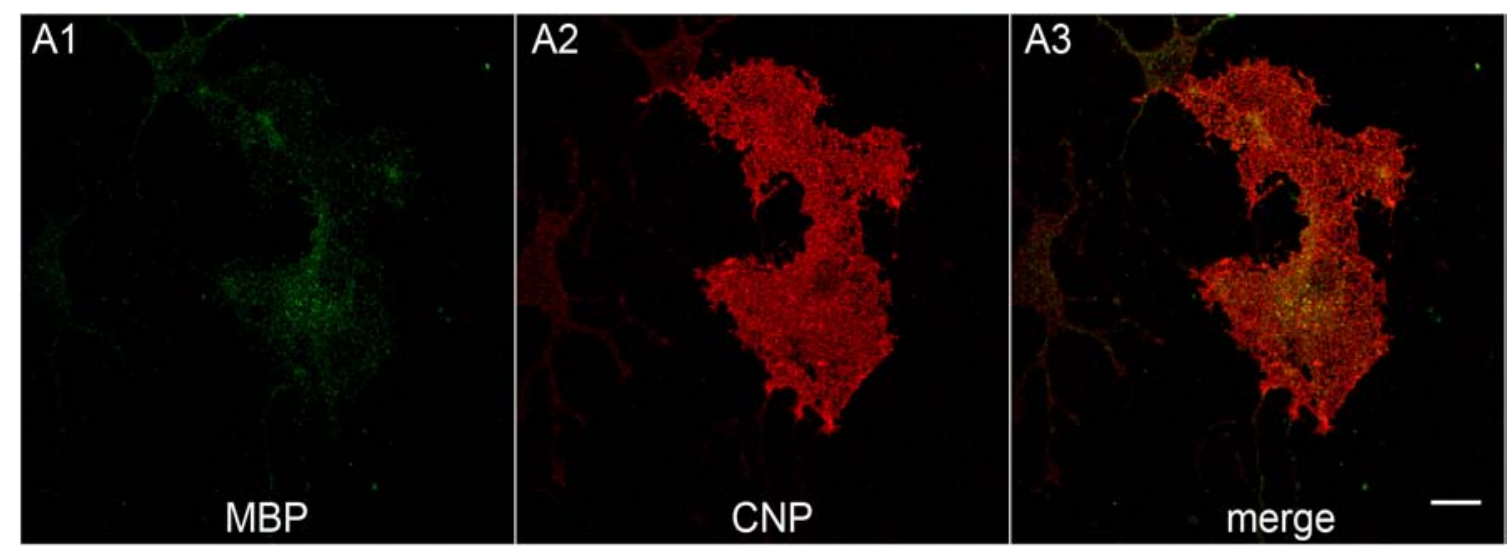

B $\%$ of cells with MBP negatve

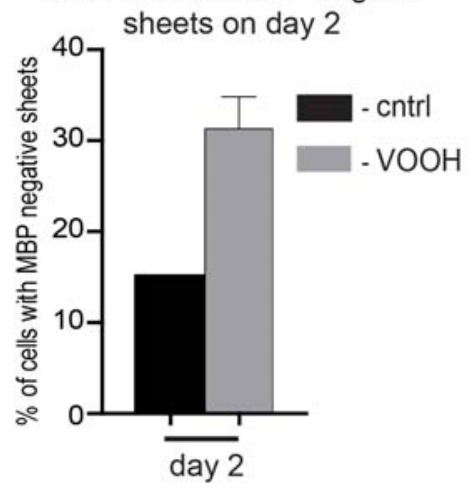

Figure 3.19: Treatment with 200 pM PTEN inhibitor leads to the increased amount of oligodendrocytes with CNP-positive, but yet MBP-negative sheets on day 2. Cells were treated with the PTEN inhibitor after 16 hours after the plating on the coverslips and fixed after 24 hours with PFA 4\% + GTA 0.25\%. After fixation cells were treated with anti-MBP (green - A1) and anti-CNP (red - A2) antibodies to determine oligodendrocytes CNP-positive and MBP-negative sheets. The percentage of cells with CNP-positive/MBP-negative sheets were calculated from the total amount of cells per coverslip (B). Upon PTEN inhibition (VOOH), there were more cells with CNP-positive and yet MBP-negative sheets at the early stage of the development, compared to the non-treated (cntrl). Data shown in the \% of the total cells. $\mathrm{N}>700$ cells per condition.

of the downstream effectors of the activated mTOR pathway and therefore might be used as an indicator of the growth pathway enhancing. Akt becomes phosphorylated upon its binding to the membrane via $\mathrm{PH}$-domain that recognises $\mathrm{PIP}_{3}$ (Wood et al., 2013). As it was expected, PTEN inhibition with VOOH resulted in increased levels of activated phosphoAkt (Figure 3.21).

Furthermore, different concentrations of the VOOH were tested to see the concentration-dependent impact on the non-compact area. As Figure 3.22 demonstrates, the difference in the area between compact and non-compact regions was detectable already upon administration of $100 \mathrm{nM} \mathrm{VOOH}$ during first $48 \mathrm{hrs}$ after shake. MBPpositive areas decrease drastically, whereas CNP-positive area of the processes increases together with the growth zone (the rim). This coincides with the increase of the phosphorylated Akt, and therefore hinted to an overall activation of the growth regulating mTOR pathway. Together with a prolonged (48 hours) treatment, a short 4 hours (acute) exposure to the high dosage of $1 \mu \mathrm{M}$ of $\mathrm{VOOH}$ on day 3 only was done. This acute treatment also resulted in the increase of the non-compact region 


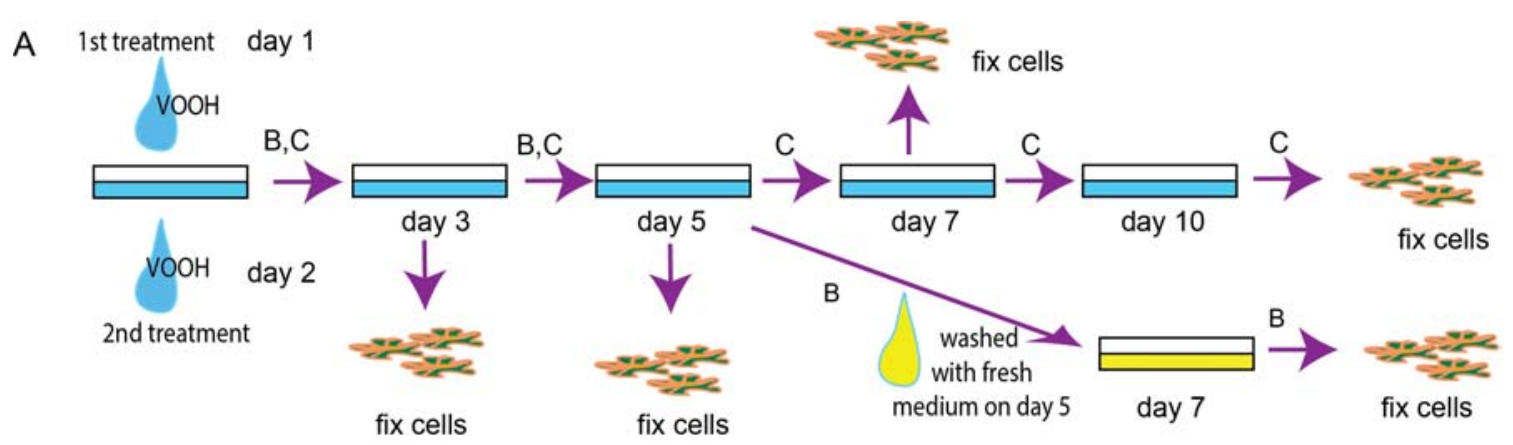

B

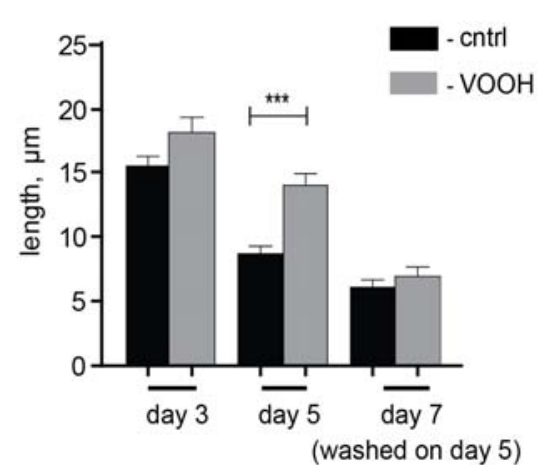

C $\%$ of area, occupied by non-compact regions

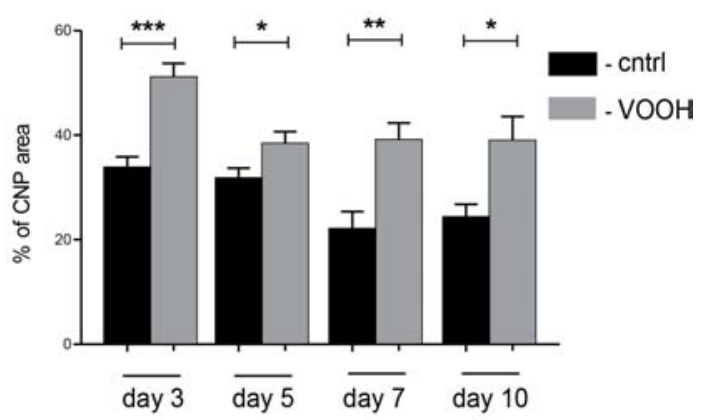

Figure 3.20: Treatment with $100 \mathrm{nM}$ PTEN inhibitor leads to an increase of rim size, as well as an increase of the non-compact regions. (A) Overview of the experiment: cells were treated repeatedly with $100 \mathrm{nM} \mathrm{VOOH} \mathrm{(blue} \mathrm{drop} \mathrm{-} \mathrm{A)} \mathrm{on} \mathrm{day} 1$ and day 2 after seeding and fixed either on day 3 (immature), or on day 5 (mature). Fresh medium (yellow drop - A) was applied to the part of the cells after day 5 and they were allowed to grow in the fresh medium till day 7 . In this case we tried to assess the reversibility of the PTEN inhibition effects. Batches of cells from day 3 , day 5 , day 7 and day 10 were fixed with PFA $4 \%+$ GTA $0.25 \%$ and analysed for morphological changes. Letters above the arrows indicate which graph corresponds to the experimental setup. After fixation cells were treated with anti-MBP and anti-CNP antibodies to determine compact and non-compact regions, respectively. (B) Rim size (growth zone) increases on day 3 and day 5 upon treatment with $100 \mathrm{nM} \mathrm{VOOH,} \mathrm{in} \mathrm{comparison} \mathrm{to} \mathrm{the} \mathrm{non-treated} \mathrm{(cntrl)} \mathrm{cells.} \mathrm{Mean} \pm$ s.e.m. of the rim size $(\mu \mathrm{m})$. (C) Non-compact myelin area is increased in the late developmental stages of oligodendrocytes (day 7 and day 10) upon treatment with $100 \mathrm{nM}$ of VOOH, in comparison to the non-treated (cntrl) cells. Mean \pm s.e.m. of $\%$ of the area, occupied by the non-compact region (CNP as a marker). For all graphs: $\mathrm{N}=3$, minimum of 30 cells per experiment, ${ }^{* * *}=\mathrm{p}<0,001$, one-way ANOVA with Tukey post-test.

\section{(Figure 3.22,F1-F3).}

Summarising the data acquired after PTEN inhibition in the oligodendrocytes at the early stages of development, one can conclude that these observations illustrate an importance of the phosphatidylinositol homeostasis for the establishment of the proper ratio of the compact/non-compact regions. 


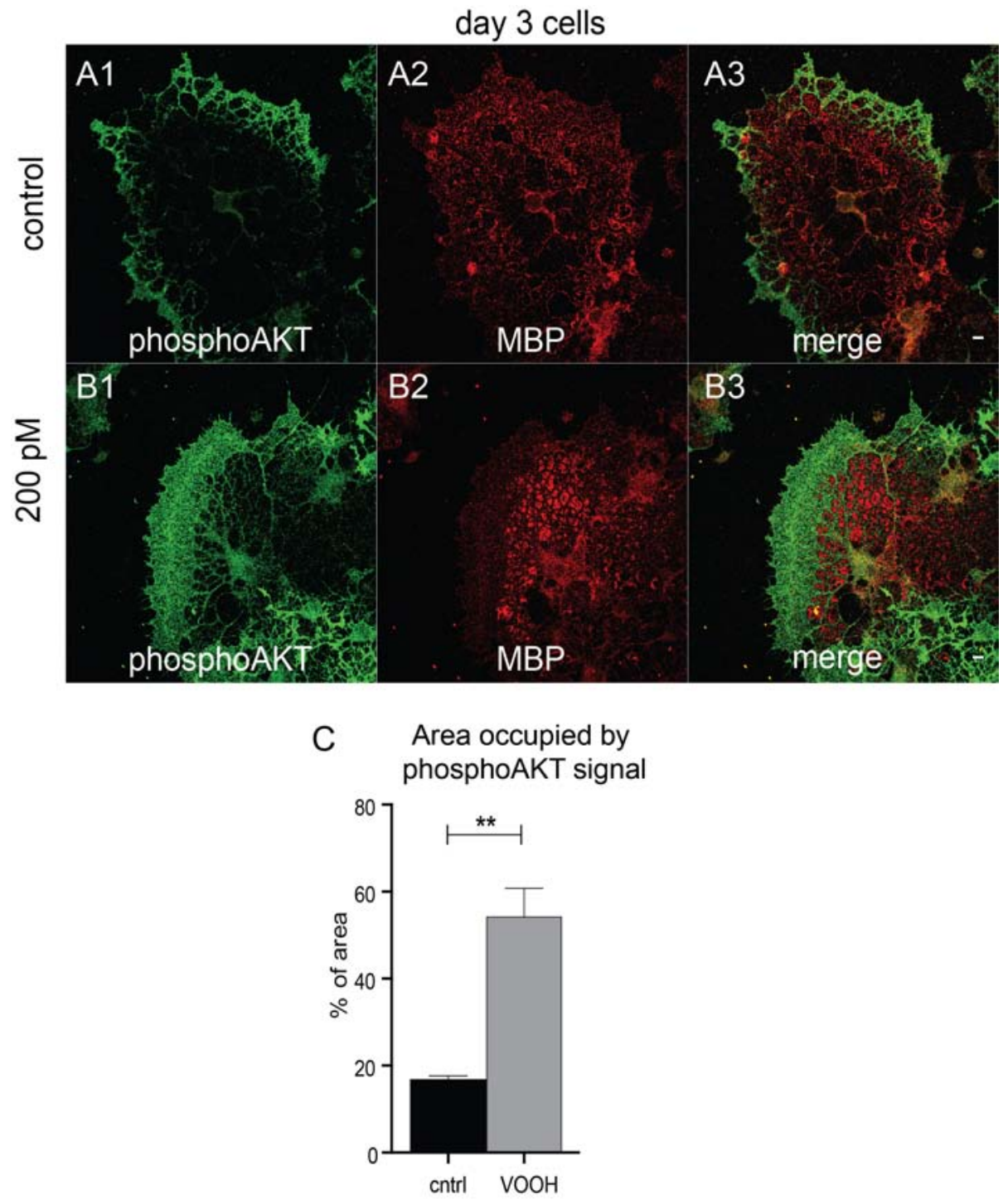

Figure 3.21: Treatment with 200 pM PTEN inhibitor leads to the increased amount of phosphoAkt in oligodendrocytes on day 3. Cells were treated on day 1 and day 2 after plating with $100 \mathrm{nM}$ VOOH and fixed on day 3 with PFA $4 \%+$ GTA $0.25 \%$. After fixation cells were treated with anti-MBP antibodies (red - A2, B2) to visualise membrane sheets, and with antiphosphoAkt (green - A1, B1). Scale bar $=10 \mu \mathrm{m}$. (C) Quantification of the cell area, occupied by phosphoAkt in $\%$ of the total cell area, with $(\mathrm{VOOH})$ and without (cntrl) PTEN inhibition. Mean \pm s.e.m. , $\mathrm{N}=2$, at least 15 cells per experiment, ${ }^{* *}=\mathrm{p}<0.01$, t-test. 

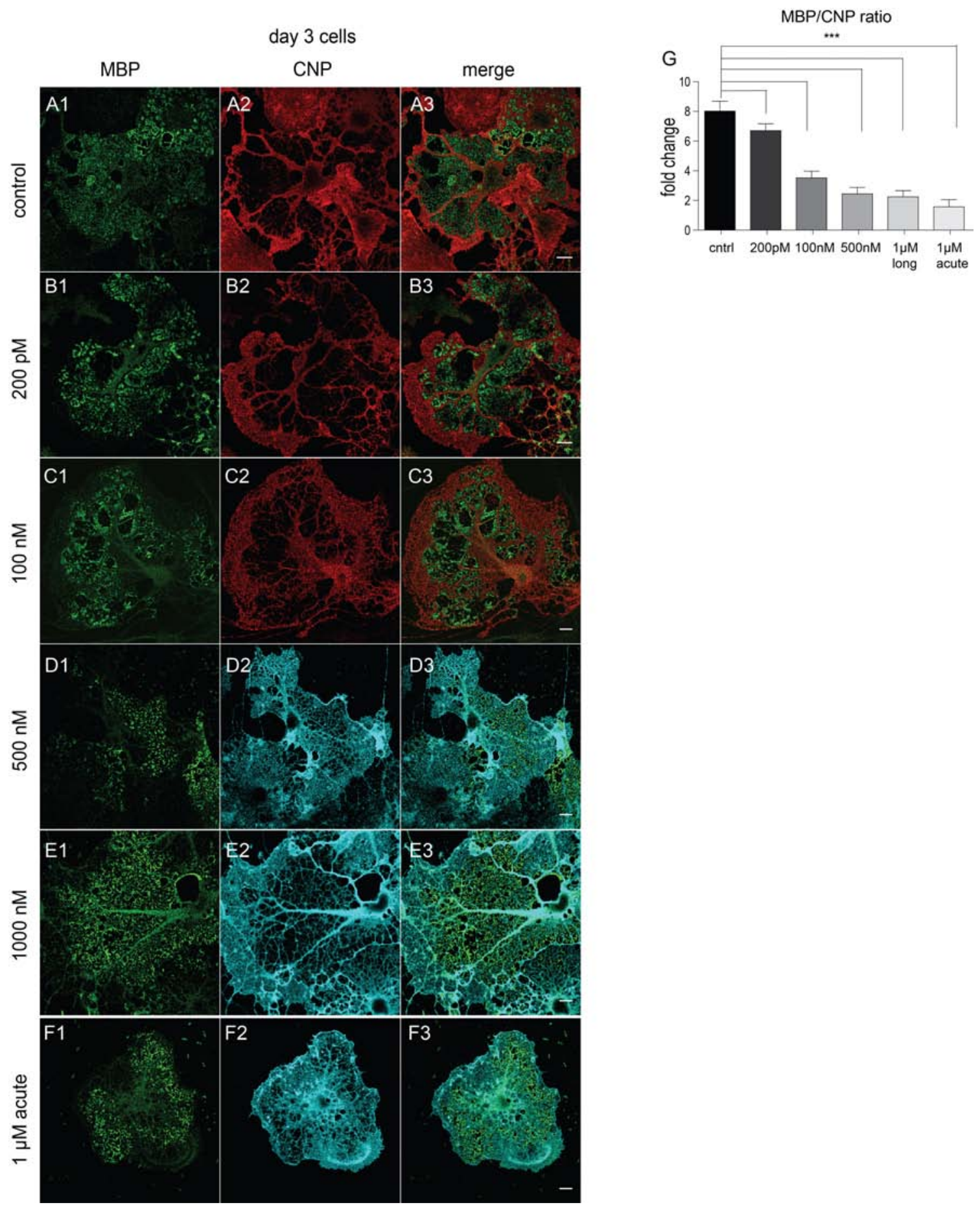

Figure 3.22: Treatment with $100 \mathrm{nM}$ PTEN inhibitor leads to the increase of noncompact region and decrease of the compact domain in oligodendrocytes on day 3 . Cells were treated on day 1 and day 2 after plating with the given concentration of the inhibitor and fixed on day 3 with PFA $4 \%+$ GTA $0.25 \%$ (A-E). Short treatment for 4 hours with high concentration $(1 \mu \mathrm{M})$ of $\mathrm{VOOH}$ was done on day 3 only and cell were fixed on day 3 with PFA $4 \%$ + GTA $0.25 \%$ (F1-F3). After fixation cells were treated with anti-MBP antibodies (green - A1F1) to visualise membrane sheets, and with anti-CNP (red - A2-C2 and cyan D2-F2) to visualise non-compact regions. Scale bar $=10 \mu \mathrm{m}$. (C) Quantification of the fold change in the MBP to CNP ratio upon different concentrations of $\mathrm{VOOH}$ and without (cntrl). Mean \pm s.e.m. , $\mathrm{N}=5$, at least 15 cells per experiment, ${ }^{* * *}=\mathrm{p}<0.001$, one-way ANOVA with Tukey post-test. 


\section{Approach 2: increase of the $\mathrm{PIP}_{3}$ amount via delivery of exogenous $\mathrm{PIP}_{3}$}

Delivery of the phosphatidylinositols with a shuttle system is an established method to study their cellular role (Tian et al., 2003). Therefore, we decided to use this approach as an alternative to the PTEN inhibition to avoid some bias from the inhibitor treatment.

Cells were subjected to the BODIPY-FL-labelled $\mathrm{PIP}_{3}$ delivery during their immature stage (day 3 ) and allowed to grow for two days (48 hours). MBP and CNP were used to visualise compact and non-compact cellular domains. Control cells were treated only with an empty cargo carrier (Figure 3.23). We didn't observe changes in the compact/non-compact ratio upon exogenous $\mathrm{PIP}_{3}$ delivery. Rim size also remained unaffected upon these conditions.

\subsubsection{Actin as a downstream effector of the $\mathrm{PIP}_{3}$ signalling}

Molecular relations between actin and $\mathrm{PIP}_{3}$ are briefly summarised in the Section 1.3.2 of the Introduction. We decided to investigate, whether increase of $\mathrm{PIP}_{3}$ in the oligodendrocytes due to the PTEN inhibition would lead to the subsequent rise in the filamentous actin levels.

First, cells were treated with different concentrations of VOOH during first 48 hours after shake (Figure 3.24 A2-A4). Additionally, a separate batch of cells was treated only for 4 hours on day 3 with high concentration $(1 \mu \mathrm{M})$ of $\mathrm{VOOH}$ (acute treatment) (Figure 3.24 A5). Filamentous actin was visualised with Rhodaminelabeled phalloidin. Both treatments did not result in any particular changes in the filamentous actin amount during the growth phase on day 3 (Figure $3.24 \mathrm{~B}$ ).

Next, cells were again treated with with different concentrations of VOOH during first 48 hours after shake and filamentous actin was visualised with Rhodaminelabeled phalloidin on day 5 (Figure 3.25 A-B). As a result, we could identify a small increase of the filamentous actin within the mature oligodendrocytes (Figure $3.25 \mathrm{C}$ ). Since filamentous actin (F-actin) usually resides within the processes and the growth zone of the cell, this finding coincides with the previous observation of the increased outer rim and processes upon PTEN inhibitor administration at the mature stage of the oligodendrocytes. To further support this observation, we performed a biochemical extraction of the two distinct fractions of the actin (globular and filamentous) at the stages of the active growth and complete maturation (Figure $3.25 \mathrm{D})$. Similar to the immunocytochemistry data, Western blot analysis showed increased amount of F-actin in VOOH-treated mature (day 5) oligodendrocytes. 

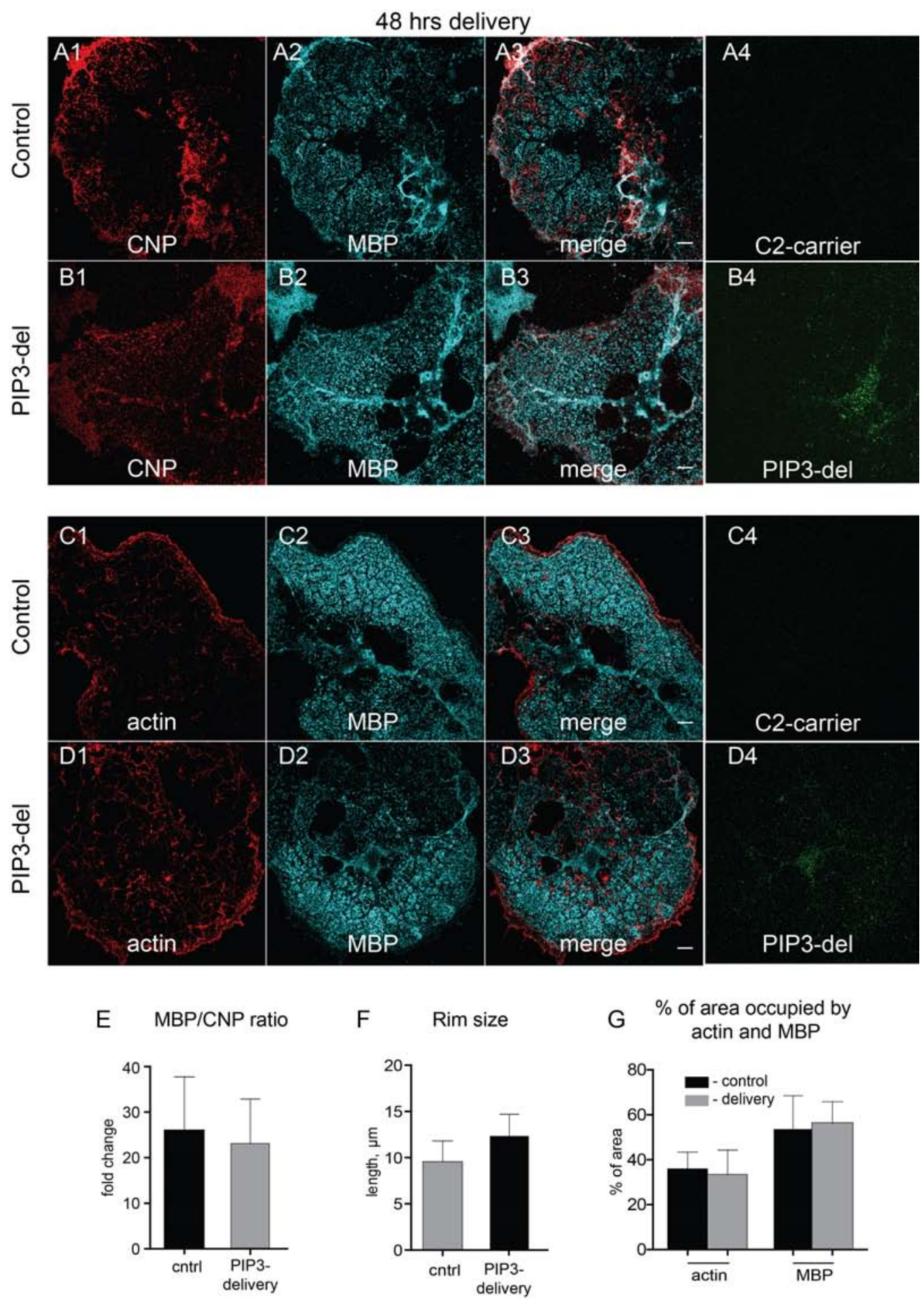

Figure 3.23: Delivery of the exogenous $\mathrm{PIP}_{3}$ did not influence neither morphology, nor actin amount in the oligodendrocytes on day 3. BODIPY-FL-PIP 3 (green - B4, D4) was delivered into cells, using Histone $\mathrm{H} 1$ as cargo-carrier (C2-carrier, non-labelled - A4,C4). Delivery was held during the active phase of the oligodendrocyte growth (day 3). Cells were allowed to reach maturation and samples were fixed on day 5 with PFA $4 \%+$ GTA $0.25 \%$. After fixation cells were treated with anti-MBP antibodies (cyan - A2-D2) to visualise membrane sheets, with anti-CNP (red - A1-B1) to visualise non-compact regions and with Rhodamine-phalloidin (red C1-D1) to visualise filamentous actin. Scale bar $=10 \mu \mathrm{m}$. (E) MBP to CNP ratio did not change upon $\mathrm{PIP}_{3}$ delivery, comparing to control (cntrl). The size of the rim was also unaffected $(\mathrm{F})$, as is the area occupied by actin $(\mathrm{G}) . \mathrm{N}=2$, minimum 10 cells per experiment. Mean \pm s.e.m. 

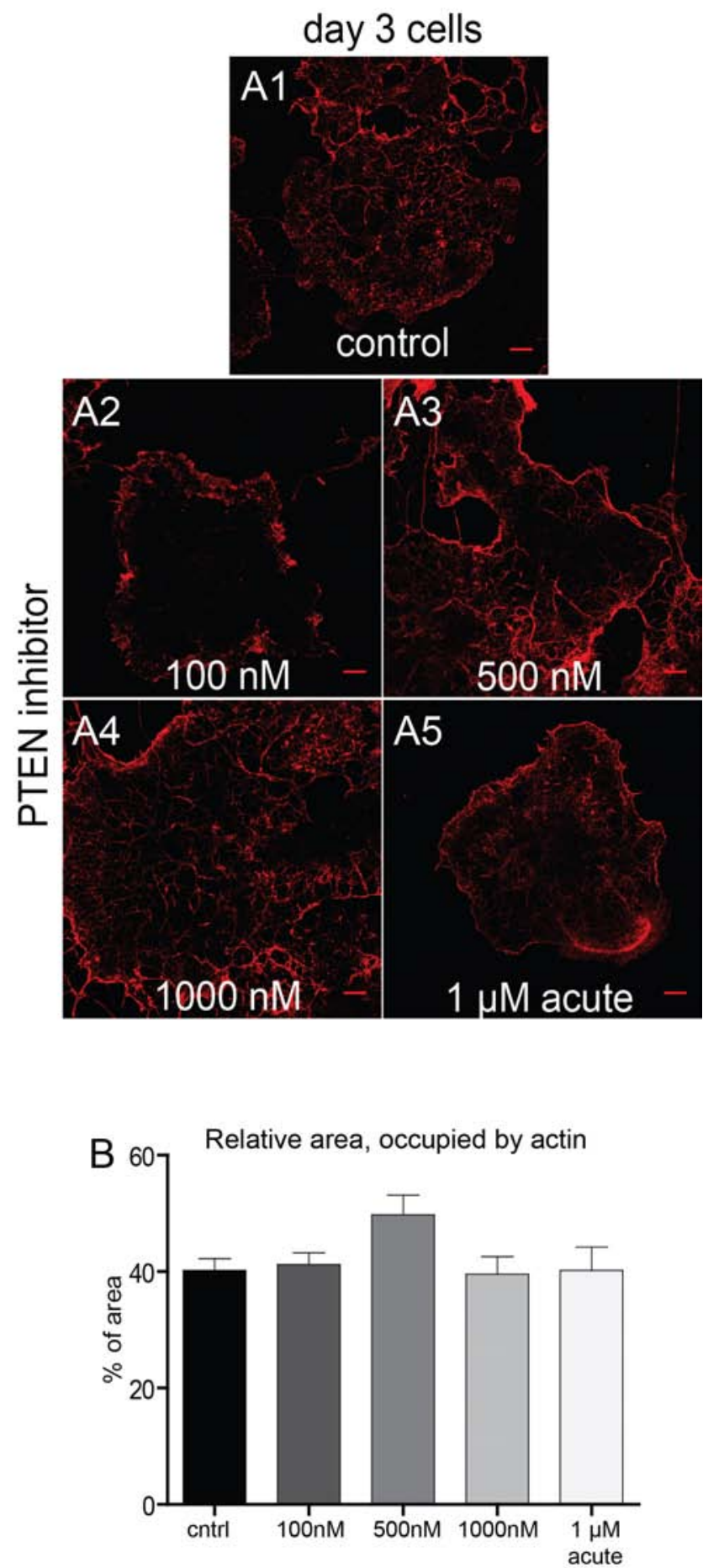

Figure 3.24: Treatment with different concentrations of PTEN inhibitor did not affect filamentous actin amount in oligodendrocytes on day 3. Cells were treated on day 1 and day 2 after plating with the given concentration of the inhibitor and fixed on day 3 with PFA $4 \%$ + GTA $0.25 \%$ (A2-A4). Acute treatment with $1 \mu \mathrm{M}$ VOOH (A5) was done only on day 3 for 4 hours, followed by immediate fixation and labelling procedure. After fixation cells were treated with Rhodamine-phalloidin to visualise filamentous actin. Scale bar $=10 \mu \mathrm{m}$. (B) Relative area, occupied by actin in \% of a total cell area upon treatment with different concentrations of $\mathrm{VOOH}$ $(\mathrm{A} 2-\mathrm{A} 5)$ and without (control $-\mathrm{A} 1) . \mathrm{N}=3$, minimum 20 cells per experiment. Mean \pm s.e.m. 

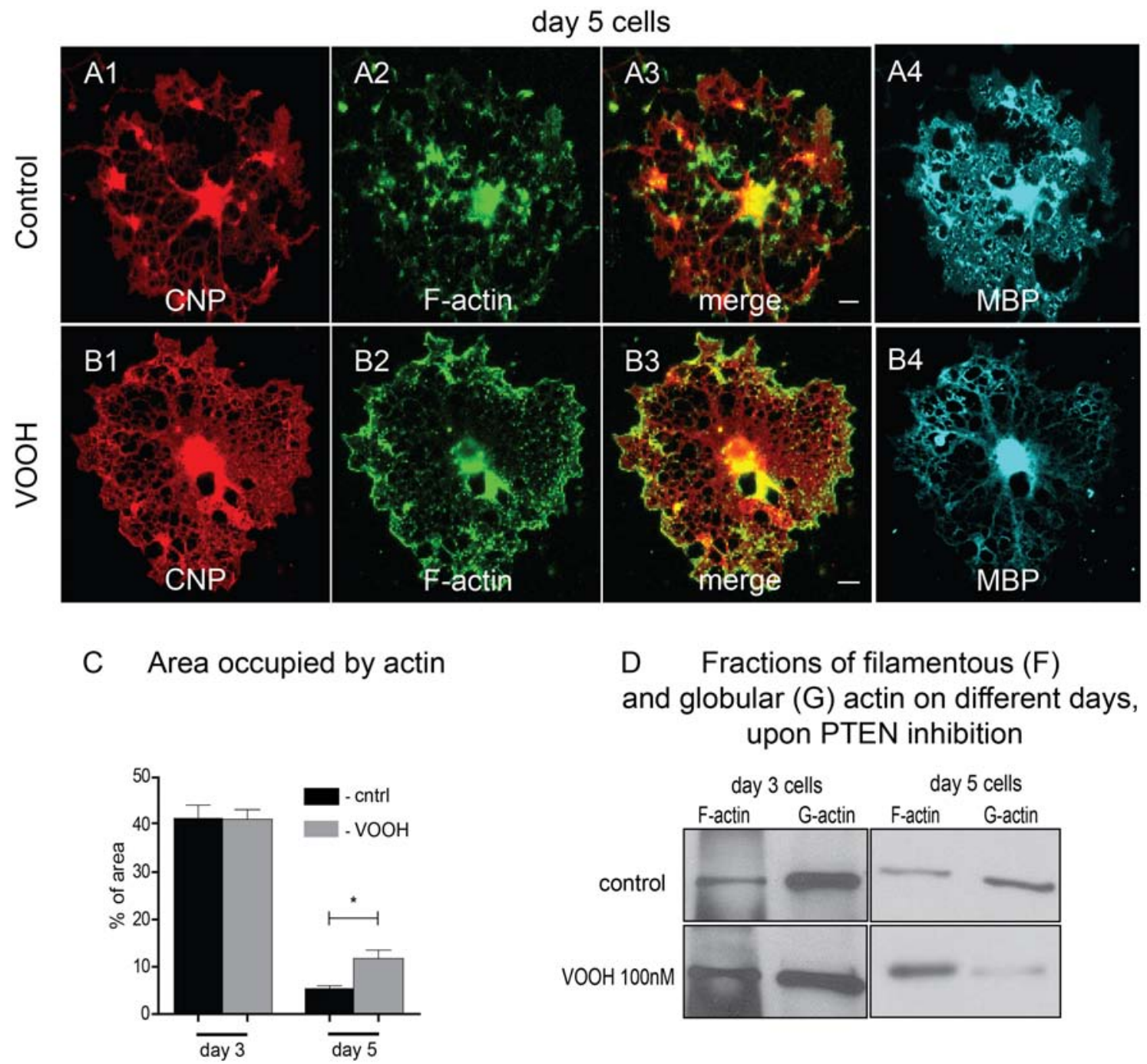

Figure 3.25: Treatment with $100 \mathrm{nM}$ PTEN inhibitor resulted in increase of filamentous actin amount in oligodendrocytes on day 5. Cells were treated consequently on day 1 and day 2 after plating with the given concentration of the inhibitor and fixed either on day 3 , or on day 5 with PFA $4 \%+$ GTA $0.25 \%$. After fixation cells were treated with anti-CNP antibody (red - A1, B1) to visualise non-compact domains, Fluorescein-phalloidin to visualise filamentous actin (green - A2, B2) and anti-MBP antibody (cyan - A4, B4) to visualise sheets. Scale bar $=10$ $\mu \mathrm{m}$. (C) Relative area, occupied by actin in \% of a total cell area upon treatment with $100 \mathrm{nM}$ of $\mathrm{VOOH}(\mathrm{A} 2-\mathrm{A} 5)$ and without (cntrl). $\mathrm{N}=3$, minimum 20 cells per experiment. Mean \pm s.e.m. , $*=p<0,05$. (D) Western blot of filamentous (F) and globular (G) actin fractions in the cells at the stage of the active growth (day 3 cells) and at mature stage (day 5 cells), with (VOOH 100 $\mathrm{nM}$ ) and without (control) PTEN inhibition. Amount of the filamentous actin is increased in the treated cells (VOOH $100 \mathrm{nM}$ ) in comparison to the control at the both developmental stages. 



\section{DisCUSSION}

Discussio mater veritas est. -

From the clash of opinions emerges the truth. 



\subsection{FCS-STED to study lipid behaviour}

Stimulated emission depletion (STED) microscopy is a process that provides super resolution by selectively deactivating fluorophores to enhance the imaging in that area. It was developed by Stefan W. Hell in 1994 (Hell \& Wichmann, 1994). In combination with fluorescence correlation spectroscopy (FCS) it is a powerful method to study dynamical behaviour of the membrane components, both lipids and proteins.

As has been mentioned before, myelin possess several unique features that distinguish it from all other membrane types. Briefly, those features are:

- high lipid-to-protein ratio with $80 \%$ of the dry weight being lipids;

- prevalence of the long-chain fatty acids within the lipid: more than 20 carbons in the chain, against $12 \mathrm{C}-16 \mathrm{C}$;

- high amount of cholesterol;

- enrichment in specific lipid species, such as plasmalogens;

- extrusion of the cytoplasm and almost all cytoplasmic proteins upon maturation by MBP compaction;

- as an important follow-up of the previous feature is an absence of actin cytoskeleton within the compact area of myelin;

- large areas of the negatively-charged lipids, sequestered by MBP.

These features of myelin make it hard to apply any of the existent models that were proposed so far to characterise membrane organisation. For example, "fence and pickets" models can not be accurate, when applied to myelin membrane, due to the absence of actin. Therefore there is a need for a new model, especially designed for myelin, taking into account all the main features known for it. To propose such a model, one first should investigate how the lipids of the myelin behave within its specific environment.

Therefore, the goal of this part of my PhD project was to collect data to make it possible to describe and to understand what are the dynamic characteristics of lipids, the major components of the myelin membrane and to suggest a model that would embrace within itself all the main myelin features.

This work was accomplished in collaboration with C. Eggeling group (department of NanoBiophotonics, Max Planck Institute for Biophysical Chemistry, Göttingen, Germany). STED-FCS setup and artificially labelled lipids used in these studies were previously described in Mueller et al. (2011) and Sahl et al. (2010).

To determine the dynamical properties of the lipids (diffusion coefficient and mode of diffusion) within the oligodendrocyte sheet we used the following Atto647Nlabelled artificial lipids, as analogs of the of the endogenous membrane lipids:

- $N$-(Atto647N)-1,2-dihexadecanoyl-sn-glycero-3-phosphoethanolamine as an analog of phosphatidylethanolamine (referred to as PE);

- $N$-(Atto647N)-sphingomyelin as an analog of sphingomyelin (referred to as $\mathrm{SM})$ 
- $N$-(Atto647N)-galactosylsphingosine as an analog of galactosylcerebroside (referred to as GalC).

These lipids were introduced into the membrane via BSA-coupled method, see Section 2.7.6 in Methods

It was previously shown that PE diffuses freely within the plasma membrane, in contrast to SM which diffusion behaviour was characterised by a strong trapping. Moreover, when other lipids with ceramide (or sphingosine) backbone were tested, their behaviour was similar to SM. Therefore, the authors suggested that ceramide (or sphingosine) backbone might be a reason, why this class of lipids encountered trapping (Mueller et al., 2011). Hydroxyl and amino groups of the ceramide function as hydrogen-bond donors close to the water-lipid interface, and might facilitate hydrogen bonds to (endogenous) membrane components, such as proteins (Pascher, 1976). Moreover, sphingomyelin diffusion and trapping was shown to be dependent on cholesterol (Mueller et al., 2011; Eggeling et al., 2009). Upon treatment with cholesterol-depleting agent, a release of SM trapping could be achieved. This was explained by the authors by the presence of cholesterol-assisted molecular complexes, that slowed down SM diffusion.

Our experiments suggest that SM in oligodendrocytes was moving faster than in PtK2 cells (Figure 3.2). This might be explained by an absence of such complexes in the oligodendrocyte sheets and more uniform distribution of cholesterol in the membrane. Furthermore, SM diffusion was reported to be dependent on underlying actin cytoskeleton: upon treatment with actin depolymerising agent (Latrunculin B), SM diffusion increased (Mueller et al., 2011; Eggeling et al., 2009).

We observed a faster diffusion for PE, SM and GalC in oligodendrocytes, when compared to PtK2 cells. Moreover, we discovered a complete absence of SM trapping in the oligodendrocyte sheets and a decreased trapping for GalC. PE showed free diffusion in oligodendrocytes, as well as in control PtK2 cells. Our findings support the suggested model of Mueller and Eggeling that endogenous SM diffusion depends strongly on actin (Mueller et al., 2011; Eggeling et al., 2009). Since oligodendrocyte sheets are deprived of actin and poor in proteins, no hindrance of the diffusion speed occurs. GalC might still show some trapping in oligodendrocytes due to the specific and so far unknown interactions in the myelin sheath.

\subsubsection{Impact of MBP on lipid diffusion}

A well-characterised system to mimic oligodendrocyte sheets in fibroblast cells was developed and reported recently (Aggarwal et al., 2013). It is based on the expression of myelin basic protein (MBP) in the PtK2 cell line that leads to the formation of the sheet-like structures. We used this PtK2-MBP + model to address the question of how MBP-membrane interactions might affect the behaviour of the lipids in the sheets. Diffusion of SM, PE and GalC in these sheet-like structures of PtK2-MBP + cells was calculated using FCS diffusion law approach(Figure 3.8) and the values were found complementing the ones derived from the oligodendrocytes.

Data from PtK2-MBP + model suggest that presence of MBP is sufficient to influence the behaviour of the lipids within the cellular membrane. Upon introducing MBP in the system, lipid behaviour changed drastically: complete disappearance of trapping for GalC and SM, together with increased diffusion coefficient for all three 
lipid species measured. It is known that MBP works as an extruder, creating a protein-poor environment in the myelin sheath (Aggarwal et al., 2013), probably leading to a decrease of obstacles for the lipid diffusion. Moreover, lack of actin leads to the absence of membrane compartmentalisation into domains that also contributes to the free diffusion mode of lipids. Therefore, presence of MBP is sufficient to promote homogenous organisation of lipids within the oligodendrocyte membrane. These data can be further extrapolated to the myelin sheath, since our cellular model was proven to bear enough similarities with its in vivo counterpart (Fitzner et al., 2006; Yurlova et al., 2011; Aggarwal et al., 2011; Aggarwal et al., 2013; Bakhti et al., 2011; Bakhti et al., 2013).

One of the myelin membrane characteristics is a high amount of cholesterol in the membrane. Diffusion speed values suggest a rather mild effect of cholesterol: all lipids tested in oligodendrocyte membrane diffused slower, in comparison to their diffusion in PtK2-MBP + cells. We hypothesise that cholesterol becomes also homogeneously distributed within the oligodendrocyte sheet membrane, rather than accumulate in specific cholesterol-rich domains, as it is the case within plasma membrane of control fibroblasts.

\subsubsection{Length of the fatty-acid chain in myelin}

One of the common features of lipids within myelin sheath is a presence of the long fatty acids in their composition, that are often over 20 carbon atoms in their length, whereas usually the plasma membrane of fibroblasts consists of mostly 16C - 18C long fatty acids. The artificial lipids, used in this study contained also 16Clong fatty acids. Therefore we measured diffusion coefficient together with mode of diffusion for an analog of the endogenous galactosylcerebroside (GalC) that contains long-chain fatty acids in its structure. We referred to this artificial lipid as 23C-GalC.

Measurement of the long-chain 23C-GalC diffusion coefficient, showed slower diffusion, in contrast to a short-chain GalC. Interesting, in contrast to short-chain GalC, 23C-GalC showed a free diffusion behaviour in PtK2 control cells. This behaviour was unexpected, since long-chain fatty acids were supposed to increase trapping of the lipid within the membrane. However, this observation might be biased due to the poor incorporation rate of the long-chain lipids into the cellular membrane. Additionally, 23C-GalC was labelled with another dye (KK114 and not Atto647N) that might also influence the results. Further experiments are needed to understand the observed free diffusion of the 23C-GalC lipid.

Therefore, we make a conclusive statement only on basis of data from short-chain lipids. We suggest an absence of constrained diffusion in oligodendrocyte sheets for the short-chain lipids. We hypothesise that studied lipids, regardless of their nature, get homogeneously distributed within oligodendrocyte sheet. Together with protein extrusion by MBP during development (Aggarwal et al., 2011; Aggarwal et al., 2013), this kind of lipid distribution creates a unique membrane structure. Such a uniform membrane organisation is favourable indeed for the processes of myelin wrapping around axons and fast delivery and spread of the newly synthesised components during the growth phase. Figure 4.1 summarise main differences between oligodendrocyte membrane and a control fibroblast plasma membrane. 

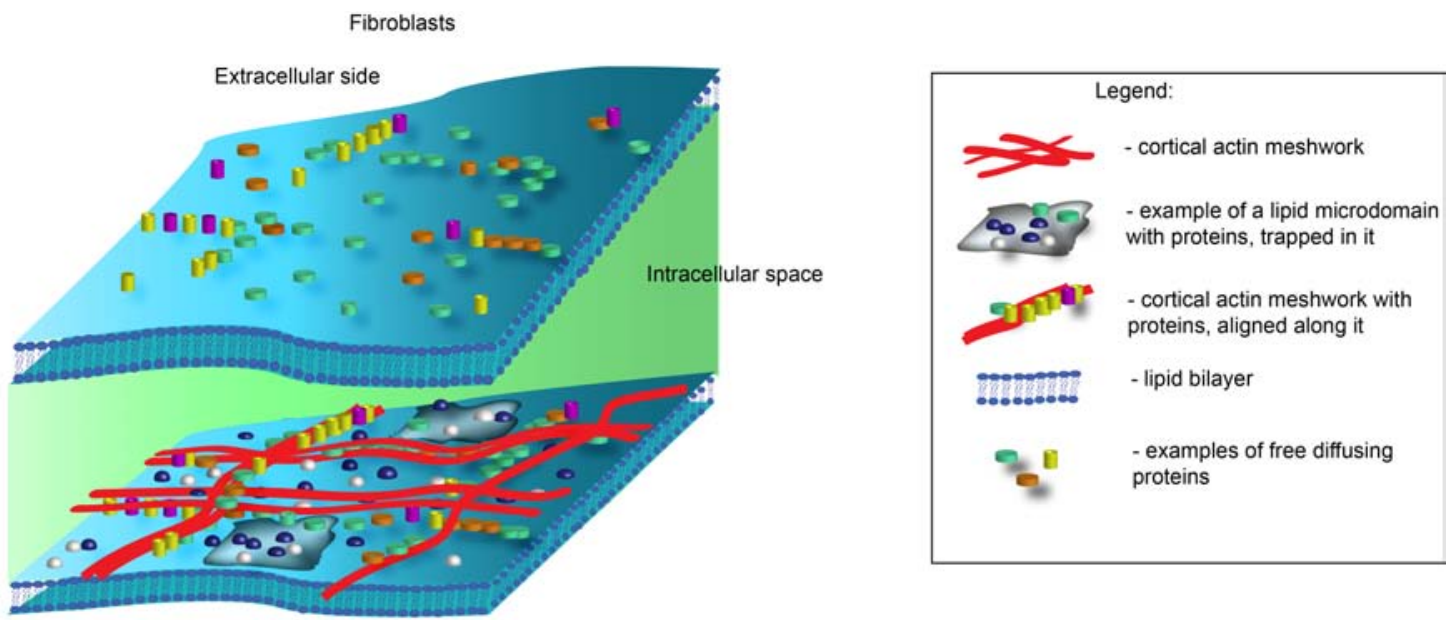

B

Sheet of oligodendrocyte
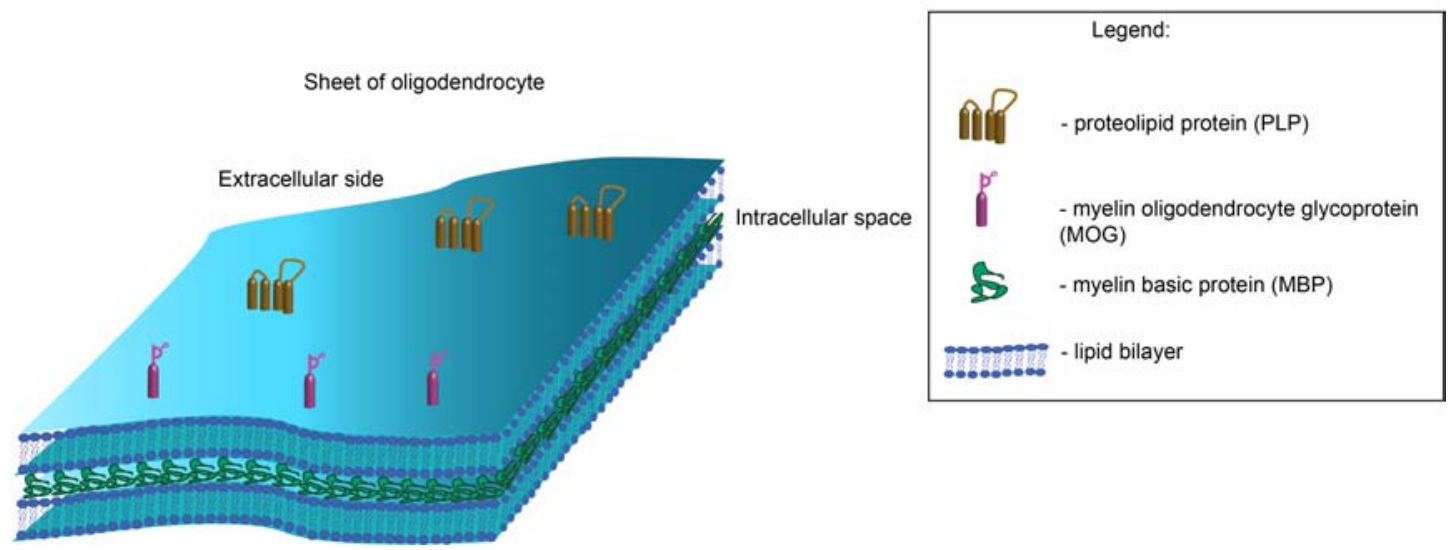

Figure 4.1: Differences between oligodendrocytes and PtK2 cell membrane. This picture summarises and compares the established differences in the lipid organisation between oligodendrocytes and plasma membrane of fibroblast cell line used in this study. (A) The common view of the normal fibroblast plasma membrane organisation is depicted: actin in red, membrane microdomains in dark blue, different proteins in the various colours and shapes. As one can see, the membrane is crowded with tremendous amounts of interaction taking place at a given time point, all influencing in a different way the diffusion of the lipids. (B) To compare: the schematic view of the myelin membrane organisation: MBP (green) zips the two adjacent membrane sheets, extruding cytoplasm and proteins with long cytoplasmic domains, creating a unique membrane plateau with a scarce impregnation of the transmembrane proteins (PLP - brown, or MOG - magenta). Such homogeneous membrane array does not oppose free membrane diffusion of the constituent lipids. 


\subsection{Role of phosphatidylinositols in the cellular po- larity}

Cellular polarity of the plasma membrane is a potent and important regulatory mechanism, involved in many crucial events, from the cellular migration, tissue formation and differentiation during development, to the more minor cases, such as epithelium function maintenance, blood cell migration, cellular division, etc. The brief overview of the polarity establishment prerequisites is given in the Section 1.6 of the Introduction. Phosphatidylinositols play an important role during cellular polarisation. Spatial and functional separation of these lipids is very broad. The tip of the growing axon is enriched with $\mathrm{PIP}_{3}$ (Shi et al., 2003), as does leading edge in neurons and migrating cells. In contrary, $\mathrm{PIP}_{2}$ is strictly sequestered to the trailing region of the migrating cells. In the epithelia, $\mathrm{PIP}_{3}$ is usually restricted to the basolateral part, while $\mathrm{PIP}_{2}$ is found in the apical part. Local changes in these phosphoinositides moieties within cellular membrane is acquired via distinct localisation of the two enzymes: phosphoinositide 3-kinase (PI3K) and phosphatase and tensin homolog (PTEN) phosphatase. PI3K generates phosphatidylinositol-3,4,5triphosphate $\left(\mathrm{PIP}_{3}\right)$ and PTEN removes the 3/-phosphate from $\mathrm{PIP}_{3}$ to generate phosphatidylinositol-4,5-bisphosphate $\left(\mathrm{PIP}_{2}\right)$. These two enzymes are mutual antagonists (Sasaki et al., 2009) and they follow the respective pattern of the separation of phosphatidylinositols: PI3K is localised to the basolateral domain and the tip of the growing axon and PTEN resides in the apical part of the epithelial cells and in the trailing region of the migratory cells (Shewan et al., 2011).

The most direct study to illustrate the importance of the $\mathrm{PIP}_{3}$ and $\mathrm{PIP}_{2}$ segregation to a respective polar domain of the cell was performed by Gassama-Diagne and co-workers (Gassama-Diagne et al., 2006). In this study, they applied an exogenous $\mathrm{PIP}_{3}$ to the apical surface of the epithelial cells. Strikingly, this alone was able to force the cell to change the identity of the apical part to the basolateral. This change was characterised by recruitment of PI3K and other basolateral protein to a former apical region, concomitant with the exclusion of apical proteins. When cells were treated with PI3K inhibitors (causing decreased $\mathrm{PIP}_{3}$ levels), they were forced to diminish in height, due to the shrinkage of the lateral sides.

Interesting studies of the role of distribution of phosphatidylinositols during cellular polarisation were done using MDCK cells. These cells, when grown in threedimensional culture, form polarised cysts with a centrally located apical surface and fluid filled lumen (O'Brien et al., 2002). The work of Martin-Belmonte and co-workers provided interesting results on how the ability of these cells to form apical domains might be affected (Martin-Belmonte \& Mostov, 2007). PTEN and PIP 2 both become specifically localised to the apical surface of the MDCK cells during early events of the cyst formation. Upon inhibition of PTEN activity, segregation of phosphatidylinositols becomes awry, leading to the disruption of lumen formation. The further delivery of the exogenous $\mathrm{PIP}_{2}$ to the basolateral surface of the cysts resulted in the lumen shrinkage and recruitment of apical membrane proteins, following the change of the membrane identity from basolateral to apical. Despite the striking findings from the mentioned studies, it is important to note that $\mathrm{PIP}_{3}$, being a common second messenger, may reside in a small portion throughout the plasma membrane. However its function there is distinct from the polarity regulation (Shewan et al., 
2011; Czech, 2000; Prehoda et al., 2000; Rohatgi et al., 2000).

Oligodendrocytes in the CNS are morphologically polarised into two functional domains: compact regions that enwrap axons and produce myelin sheath and noncompact parts that enable connectivity of myelin membrane to the cell body and are responsible for trafficking of the membrane components and molecular nutrients delivery to the compact areas parts. Taking into account a role of $\mathrm{PIP}_{2}$ and $\mathrm{PIP}_{3}$ in majority of the cells, we questioned whether these lipid species are also differentially localised within oligodendrocytes.

\subsubsection{Mature oligodendrocytes show distinct localisation of the phosphatidylinositols}

Mouse oligodendrocytes grown on glass coverslips develop flat membrane extensions that mimic the biochemical and morphological composition of myelin sheath in vivo (Aggarwal et al., 2011; Aggarwal et al., 2013; Bakhti et al., 2011; Fitzner et al., 2006). Moreover, it was described that these plasma membrane extensions are polarised into distinct membrane domains, resembling compact and non-compact myelin. These domains develop without a need to wrap around the axon, illustrating sufficient intrinsic cellular mechanisms within the oligodendrocytes. Possibility to cultivate primary oligodendrocytes in the absence of axon enables the localisation of molecules with the light microscopy.

First, we determined localisation of $\mathrm{PIP}_{2}$ and $\mathrm{PIP}_{3}$ within the membrane of the oligodendrocytes. Using several immunolabelling-based approaches, we managed to demonstrate the following: $\mathrm{PIP}_{2}$ is enriched in the oligodendrocyte sheet (compact region) and $\mathrm{PIP}_{3}$ is restricted in the processes and outer rim (non-compact region) (Figure 3.11).

It was previously demonstrated by independent studies that MBP has an ability to sequester $\mathrm{PIP}_{2}$ (Musse et al., 2008; Harauz et al., 2000). Furthermore, it was suggested that $\mathrm{PIP}_{2}$ and MBP interaction are important to stabilise MBP association with the membrane (Nawaz et al., 2009). In our study we demonstrated that MBP interacts with $\mathrm{PIP}_{2}$ in the oligodendrocyte sheets, masking it from the antibody recognition: $\mathrm{PIP}_{2}$ presence in the sheets could be visualised only upon detaching of MBP from the cytosolic part of the plasmalemma. However, MBP detachment from the membrane has no influence on the $\mathrm{PIP}_{3}$ detection. Based on these observations, we concluded that MBP sequesters $\mathrm{PIP}_{2}$, but not $\mathrm{PIP}_{3}$ in the oligodendrocyte sheets.

\subsubsection{Early-stage oligodendrocytes, and those from shiverer mice, lack differential localisation of the phosphatidyl- inositols}

Next, we addressed the question when does the differential localisation of phosphatidylinositols take place. Immunolabelling analysis of the early-stage oligodendrocytes showed an absence of the strict separation of phosphatidylinositols when MBP was yet absent and sheets were non-compacted. Nevertheless, $\mathrm{PIP}_{3}$, but not $\mathrm{PIP}_{2}$, showed a slight enrichment towards the outer parts of the cell, already on the early stage.

As was concluded in the Part I of this work, lipids diffuse free within the oligodendrocyte sheet. Therefore, if $\mathrm{PIP}_{3}$ diffusion becomes restricted, there should be 
a mechanism to exhibit this confinement. Since $\mathrm{PIP}_{3}$ interacts with a broad range of the $\mathrm{PH}$-containing proteins, these interactions might restrict the free diffusion of $\mathrm{PIP}_{3}$ from the outer rim.

One also might suggest that MBP is a force that separates $\mathrm{PIP}_{2}$ and $\mathrm{PIP}_{3}$ into distinct pools within the membrane on the later stages. We studied the localisation of phosphatidylinositols in the shiverer mice, where no MBP is present even at the mature stages. We found that in shiverer mouse oligodendrocytes, $\mathrm{PIP}_{2}$ and $\mathrm{PIP}_{3}$ have the same localisation, as in the early stage oligodendrocytes: absence of the strict phosphatidylinositols separation with slight enrichment of $\mathrm{PIP}_{3}$ towards the outer parts of the cell (Figure 3.16). Therefore, MBP is necessary to separate phosphatidylinositols.

\subsubsection{MBP as a phosphatidylinositols'-separating protein}

From the data of the liposomal pull-down, there are no preferences in binding of MBP towards any of phosphatidylinositols tested. It might suggest, therefore, that binding and masking of $\mathrm{PIP}_{2}$ by MBP takes place primarily due to the initial spatial separation of phosphatidylinositols: $\mathrm{PIP}_{3}$ enrichment in the growth zone region, and $\mathrm{PIP}_{2}$ residing in the sheets. This spatial separation occurs on the early stage of the cellular development, before MBP appears in the sheets. Subsequently, when MBP starts to zip the sheets, it interacts with $\mathrm{PIP}_{2}$ that leads to $\mathrm{PIP}_{2}$ sequestration in the sheet region.

$\mathrm{PIP}_{2}$ is one of the most abundant phosphatidylinositol species present in the inner leaflet (up to $2 \%$ ) where it is involved into ample amount of cellular processes (Czech, 2000; McLaughlin et al., 2002). In contrary, amount of $\mathrm{PIP}_{3}$ in the resting cell is scarce, and even upon activating signals it does not rise more than $1 \%$ of the total lipid amounts (Stephens et al., 1993). Despite its little amount, $\mathrm{PIP}_{3}$ is one of the major signalling molecules within a plasmalemma, a potent growth and survival signal (Bohdanowicz \& Grinstein, 2013).

One of the pathways under $\mathrm{PIP}_{3}$ tight regulation is the $\mathrm{PI} 3 \mathrm{~K} / \mathrm{Akt} / \mathrm{mTOR}$ pathway, involved in cell growth, proliferation and survival. PH-domain of the Akt binds the membrane $\mathrm{PIP}_{3}$ and enables the proper positioning of Akt on the membrane for its further phosphorylation and cascade onset. In contrary, dephosphorylation of $\mathrm{PIP}_{3}$ by PTEN has an inhibitory effect on this pathway (Wood et al., 2013). $\mathrm{PI} 3 \mathrm{~K} / \mathrm{Akt} / \mathrm{mTOR}$ pathway involvement in the differentiation of oligodendrocytes and myelination onset has been already described in several studies (Narayanan et al., 2009; Tyler et al., 2011; Guardiola-Diaz et al., 2012).

The most outer rim of the oligodendrocytes, where the $\mathrm{PIP}_{3}$ enrichment was shown, was suggested to play a role of a growth zone, where all the necessary growth pathway constituents are gathered. We suggest that early in the development accumulation of $\mathrm{PIP}_{3}$ occurs in this outer most rim, followed by its recognition by Akt. After binding to a specific region of the plasma membrane, Akt gets phosphorylated and its activated form triggers the growth pathway (Wood et al., 2013).

In contrary, $\mathrm{PIP}_{2}$ is spread over the whole plasmalemma (McLaughlin et al., 2002). However, due to the natural diffusion, both phosphatidylinositols species intermix, even though the enrichment of $\mathrm{PIP}_{3}$ in the rim and elimination of $\mathrm{PIP}_{3}$ in the sheets might be under surveillance of PI3 kinase and PTEN, respectively.

Differentiation and growth of the oligodendrocytes in culture starts immediately 
after their attachment to the poly-L-Lysine (PLL)-coated coverslips (several hours after shake of the cells from the tissue flasks). MBP translation and compaction of the sheet follows with a $2-3$ days delay and is mostly completed on the day 5 . Growth on the rim side and compaction, thus, both occur at a certain time window simultaneously. Feature of the MBP translation is the transport of its mRNA via hnRNP granules to the sites of its expression. Upon release from the ribosomes in the processes, MBP gets attracted to the negatively charged membrane where it recognises $\mathrm{PIP}_{2}$ (and most likely phosphatidylserine). Upon reaching the crucial concentration and due to its intrinsic properties, MBP molecules undergo a phase transition into a tight network (Aggarwal et al., 2013). Proceeding in this step-wise way (interaction with $\mathrm{PIP}_{2}$, followed by a phase transition into a sieve-like structure), MBP zips two adjacent membranes together, extruding most of the proteins and rearranging lipids and forming a unique compact sheet (Aggarwal et al., 2011; Aggarwal et al., 2013; Yurlova et al., 2011).

The suggested cascade of the events occurring from the early phase of the noncompact oligodendrocytes to a full sheet closure in a mature state is summarised in the Figure 4.2.

Data acquired from the shiverer oligodendrocytes, where phosphatidylinositols separation does not occur due to the lack of the MBP and, subsequently, absence of the compact zone formation, support this model. Therefore, shiverer oligodendrocytes reside in the state, shown on the Figure 4.2 A.

The growing part of the cell is in a constant need of the molecular transport and protein synthetic machinery. Membrane sheets are, however, deprived of it (Aggarwal et al., 2011; Aggarwal et al., 2013). Therefore, there should be a signalling pathway involved to keep the growth zone of the cell non-compact. We suspect $\mathrm{PIP}_{3}$ enrichment within the growth zone might play a role in this. To test this assumption, we decided to intervene with phosphatidylinositols levels by increasing amounts of one of the phosphatidylinositol species, namely $\mathrm{PIP}_{3}$.

\subsubsection{Compact/non-compact domain ratio can be altered upon augmentation of $\mathrm{PIP}_{3}$ in the oligodendrocytes}

Proper functioning of the cell highly depends on the phosphatidylinositols homeostasis: PTEN inhibition leads to the increased $\mathrm{PIP}_{3}$ accumulation, hyperactivation of Akt-pathway. As a result, such a malfunction of the growth regulation leads to cancer (Sasaki et al., 2009; Chalhoub \& Baker, 2009). We decided to shift phosphatidylinositols' equilibrium to follow possible changes in the cellular morphology and MBP compaction behaviour. To achieve the shift, we used two independent approaches.

Approach 1: $\mathrm{PIP}_{3}$ increase due to PTEN inhibition. Vanadium tryhidarte compound (VO-Ohpic, further referred to as $\mathrm{VOOH}$ ) was reported to be potent and highly selective inhibitor of PTEN (Rosivatz et al., 2006; Nakai et al., 2004). Therefore, we used it to increase $\mathrm{PIP}_{3}$ amount in the oligodendrocytes. We confirmed a success of the PTEN inhibition by an increase in the $\mathrm{PIP}_{3}$ and phosphorylated Akt (activated form of the Akt) signals via immunostaining (Figure 3.18 and Figure 3.21).

Normally, during maturation, non-compact domain zone decreases proportionally 
A

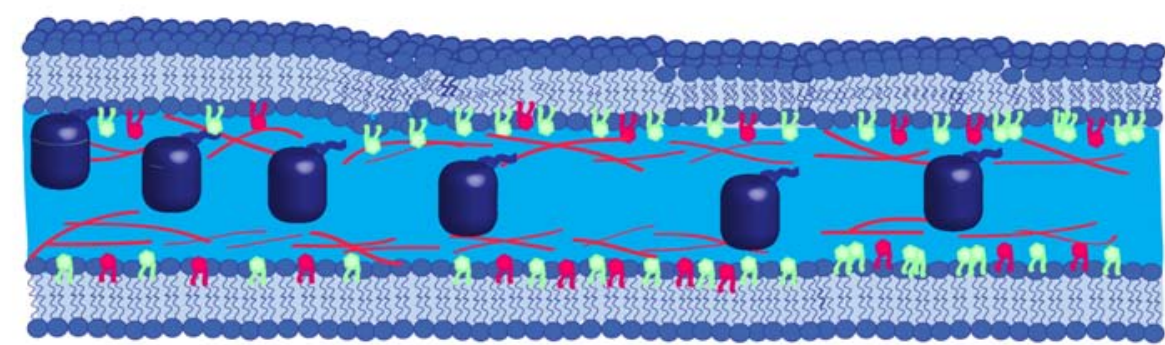

B

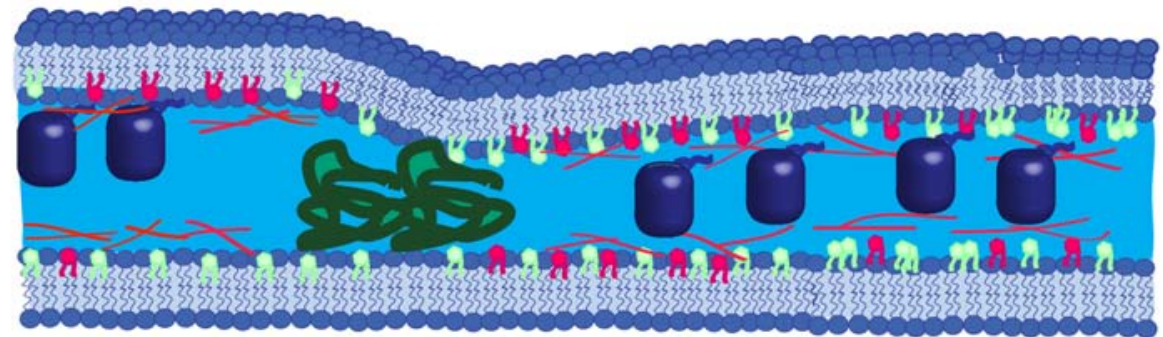

C

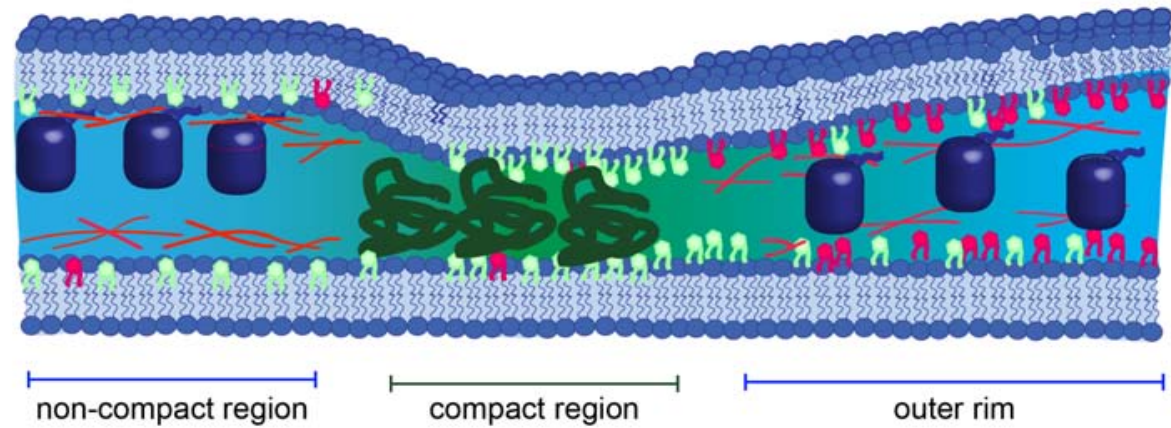

Legend:

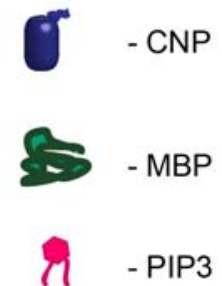

- PIP2

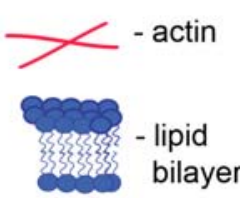

Figure 4.2: Model of phosphatidylinositols unmixing by MBP. (A) $\mathrm{PIP}_{3}$ (magenta) is more enriched within so called growth zone at the rim, where it most likely takes part in the mTOR signalling pathway, enabling sheet to grow further. Due to the unrestricted lipid diffusion in the sheets with no MBP at the early stages of development, $\mathrm{PIP}_{3}$ diffusion occurs and $\mathrm{PIP}_{3}$ could be identified within the sheet regions as well. $\mathrm{PIP}_{2}$ (lime), as a general component of the cellular membrane, is more uniformly distributed over the cell. Sheets are filled with CNP (dark blue) and other proteins (not shown on a sketch) at this stage. (B) MBP translation begins, followed by MBP interaction with the negatively-charged lipids of the inner membrane leaflet; next, two inner leaflets are brought together and zipped. (C) An extrusion of CNP and other lipids from the sheets follows. Upon binding of MBP to the $\mathrm{PIP}_{2}$ in the sheet region, the masking of the latter from antibody recognition occurs. The $\mathrm{PIP}_{3}$ residues might also take part in the MBP-membrane binding, even though they are scarce in these regions. Newly generated $\mathrm{PIP}_{3}$ molecules are now restricted to the place of their synthesis (rim and process).

to the increase of the MBP-positive zone. Mature cells on the day 5 have a small portion of the processes and a thin outer rim with small growth zone. On even later stages, non-compact zone resides only within the tiny processes that close completely when cells are cultivated longer than $7-8$ days in culture (Aggarwal et al., 2011).

Upon PTEN inhibition with VOOH we observed enlarged rim and non-compact areas in the mature cells (Figure 3.22). Moreover, inhibition of PTEN at the early stages of development led to a decrease of the compact zone. This effect was concentration-dependent (Figure 3.22). Albeit, after removal of the PTEN inhibition (via washing and adding a fresh medium) cells could restore a normal profile of the compact/non-compact domain (Figure 3.20 C).

Furthermore, application of the PTEN inhibitor immediately after cell seeding 
on the coverslips could force the cells to expand non-compact sheets faster, in comparison to the untreated cells (Figure 3.19).

Approach 2: $\mathrm{PIP}_{3}$ increase due to the exogenous $\mathrm{PIP}_{3}$ delivery. Any application of the inhibitor is a stress for the cell, since it could affect some of the survival pathways. In addition, every cell type reacts slightly differently to the treatment. Therefore, we decided to deliver exogenous $\mathrm{PIP}_{3}$ via a shuttle system as another method to increase $\mathrm{PIP}_{3}$ levels. Cells were subjected to the BODIPY-FL-labelled $\mathrm{PIP}_{3}$ delivery at their active growth stage (day 3) and fixed at the mature stage (day 5). In this particular experimental setup delivery was carried out for 48 hours, to reproduce time window for the PTEN-inhibition (also $48 \mathrm{hrs}$ ). We did not observe any cellular phenotype using this method. One reason for this might be a low concentration used to achieve a response from the oligodendrocytes. Another reason could be a fast dephosphorylation of the exogenous BODIPY-FL-PIP 3 , as was previously observed by Tian and co-workers (Tian et al., 2003). Hence, one might consider repetition of the delivery and establishing a precise window for the oligodendrocyte cellular response, as well as a proper concentration of the BODIPY-FL-PIP . $_{3}$

\subsubsection{Increased levels of $\mathrm{PIP}_{3}$ and actin might regulate the size of the non-compact domains}

A link between increased $\mathrm{PIP}_{3}$ level and enlarged non-compact domains was discussed in the previous sections. We suggest that $\mathrm{PIP}_{3}$ might have a signalling role for the MBP to prevent the compaction. Since electrostatic interactions between $\mathrm{PIP}_{3}$ and MBP occur in vitro (as shown by the liposomal pull-down experiment (Figure 3.23), there might be a physical obstacle that prevents interaction between $\mathrm{MBP}$ and $\mathrm{PIP}_{3}$ in the plasma membrane in vivo, resulting in the termination of compaction at the rim. One of the suggested candidate to serve as such an obstacle is a filamentous actin.

Increase in the actin amount in the regions, enriched with $\mathrm{PIP}_{3}$ might be expected, due to the tight regulation between $\mathrm{PIP}_{3}$ amount and cytoskeleton rearrangements discussed in the Introduction Section 1.3.2 of this work. We also could detect an increase of the filamentous actin amounts at the mature stage of oligodendrocytes ' development upon VOOH treatment (Figure 3.24 and 3.25). This is an interesting finding, since filamentous actin depolymerisation was described to be a prerequisite for the MBP compaction to take place.

Therefore, we suggest a filamentous actin to be candidate to regulate the ratio between compact and non-compact zones. At physiological conditions, actin might perturb the interactions of MBP with membrane and thus leave the growth zone non-compact. When actin levels expand, as when triggered by high levels of $\mathrm{PIP}_{3}$, MBP molecules encounter a physical obstacle and, failing to compact the sheet, most likely undergo premature degradation. This leads to the increase of the non-compact domain. For the summary of the cascade of suggested events after $\mathrm{PIP}_{3}$ levels increase in the oligodendrocytes see Figure 4.3. 
A

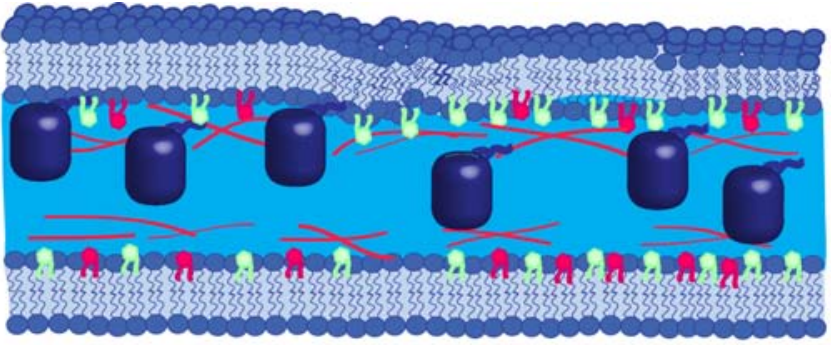

Legend:

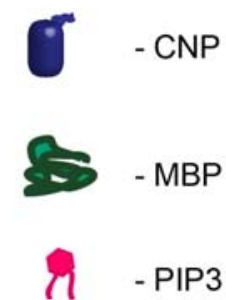

- PIP2

B
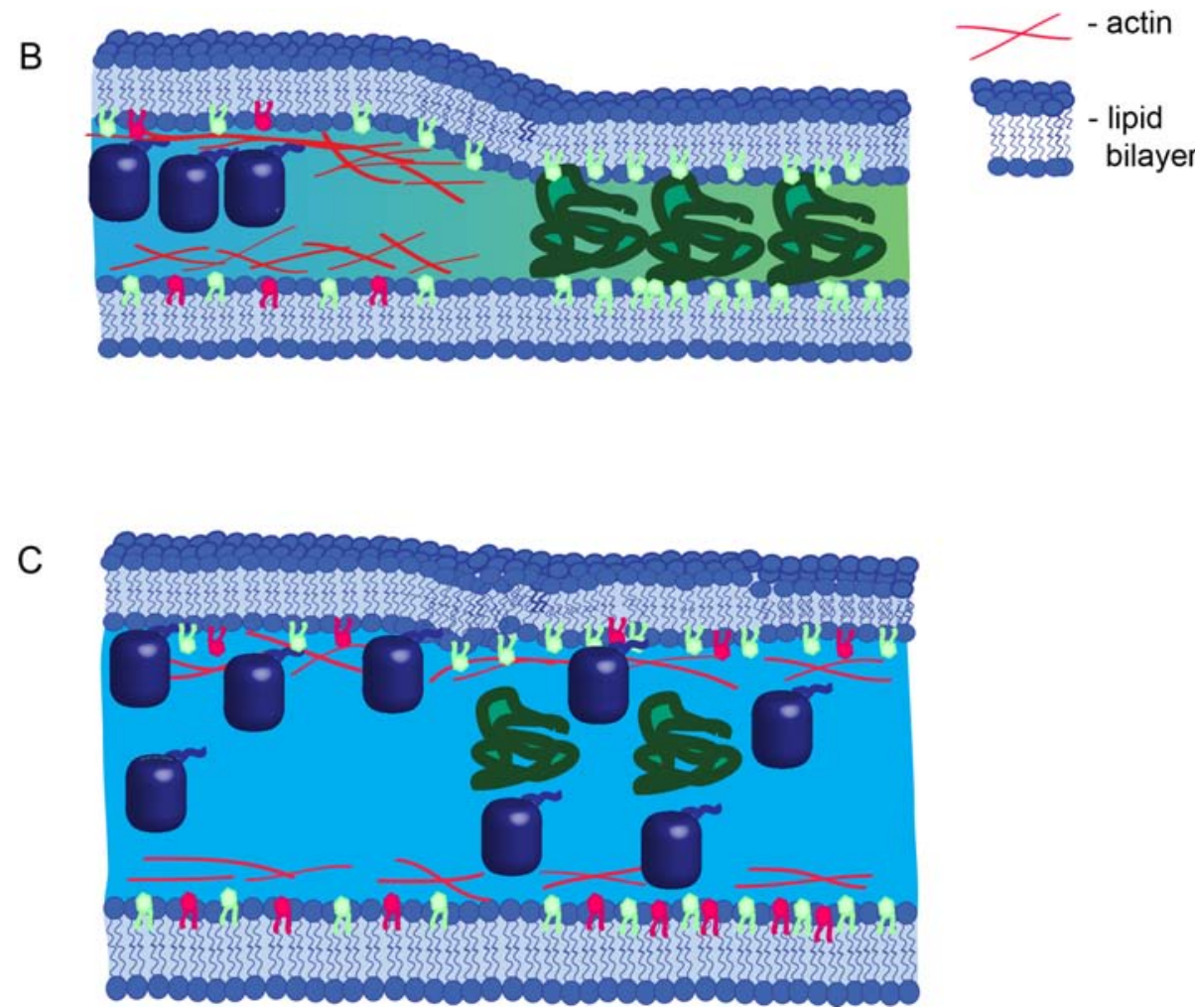

Figure 4.3: Model of the PTEN inhibition effect on the compact/non-compact domain development. (A) Representative sketch of the non-compact myelin domain. CNP (blue barrels) is filling the space. Actin cytoskeleton (red lines) is enriched under the membrane. PIP $_{2}$ (lime) and $\mathrm{PIP}_{3}$ (magenta) are mixed within the inner leaflet. (B) Following the MBP translation, compaction takes place. This results in extrusion of all the non-compact domain proteins (Aggarwal et al., 2011) and, as has been demonstrated in this work, segregates phosphatidylinositols. (C) PTEN inhibition leads to an increased cytoplasmic volume, due to the growth cascade activation; furthermore, augmented amounts of filamentous actin accumulated beneath the plasma membrane. These facts create sufficient obstacle for the MBP to establish an electrostatic interaction with the phosphatidylinositols of the inner leaflet. As a follow up, MBP gets degraded and decrease in the compact domain area is observed, together with an increase of the non-compact domains 



\section{SUMMARY AND CONCLUSIONS}

Science never solves a problem without creating ten more.

George Bernard Show 

The work presented in this thesis characterised lipid dynamics and distribution of the $\mathrm{PIP}_{2}$ and $\mathrm{PIP}_{3}$ within myelin membrane sheet. To overcome the extreme compaction and thickness of the myelin layers in vivo, we addressed our questions using primary cultures of myelin-producing cells in CNS - oligodendrocytes (Aggarwal et al., 2011; Aggarwal et al., 2013; Yurlova et al., 2011; Bakhti et al., 2011; Fitzner et al., 2006). Since this system has been proven to successfully recapitulate myelin-sheath in vivo, we suggest that acquired data could be mirrored to the in vivo situation.

To determine the dynamical properties of the lipids (diffusion coefficient and mode of diffusion) within the oligodendrocyte sheet we used STED-FCS. The fibroblast cell line, PtK2 cells, was used as a control. Following artificial lipids were used in this work, as analogs of the endogenous membrane lipids:

- $N$-(Atto647N)-1,2-dihexadecanoyl-sn-glycero-3-phosphoethanolamine as an analog of phosphatidylethanolamine (referred to as PE);

- $N$-(Atto647N)-sphingomyelin as an analog of sphingomyelin (referred to as $\mathrm{SM})$

- $N$-(Atto647N)-galactosylsphingosine as an analog of galactosylcerebroside (referred to as GalC).

These lipids were introduced into the membrane via BSA-coupled method. We found that the values for PE diffusion are similar between oligodendrocytes and PtK2 cells; however, SM and GalC diffusion coefficients were strikingly different: slow in PtK2 and fast in oligodendrocyte sheets. Furthermore we demonstrated an absence of anomalous diffusion in the plasma membrane of primary oligodendrocytes for $\mathrm{PE}$ and SM. In the control PtK2 cells, only PE was undergoing free diffusion, whereas $\mathrm{SM}$ and GalC showed strong trapping. Trapping of GalC was reduced in oligodendrocyte sheets, comparing to PtK2 control cells.

To further investigate an involvement of MBP in the lipid behaviour, we mimicked oligodendrocyte sheets in the PtK2 cells. In brief, PtK2 cells were transfected with a chimeric construct, consisting of MBP, GFP, transmembrane domain and an endoplasmatic reticulum (ER)-retention signal. Cells, transfected with this construct, were referred to as PtK2-MBP + cells. Upon introducing the sheets-like structures in the fibroblasts, diffusion of SM and GalC changed dramatically: these lipids lost their trapping, and their diffusion coefficient strongly increased. Diffusion coefficient for PE also increased in the PtK2-MBP + cells.

We concluded, hence, that MBP, due to its intrinsic characteristics, via extrusion of the cytoplasmic proteins during compaction might create a plausible homogenous environment that favours free lipid diffusion within the oligodendrocyte sheet. Such flexible constitution of this membrane might be indeed beneficial for the wrapping of myelin around the axon and for the delivery of the newly synthesised components to the fast growing membrane.

To investigate a distribution of the $\mathrm{PIP}_{2}$ and $\mathrm{PIP}_{3}$ within myelin membrane sheet, we again used primary oligodendrocyte cell culture. We determined a distinct localisation of the two phosphatidylinositols species: $\mathrm{PIP}_{2}$ enrichment in the sheet region and $\mathrm{PIP}_{3}$ localisation within the outer rim and processes. We demonstrated that different localisation of phosphatidylinositols starts at the early developmental stage, 
with $\mathrm{PIP}_{3}$ being slightly enriched in the outer cellular regions, and $\mathrm{PIP}_{2}$ broadly distributed over the cellular membrane. Upon MBP expression, $\mathrm{PIP}_{2}$, but not $\mathrm{PIP}_{3}$, got sequestered and masked by MBP within sheets regions, whereas $\mathrm{PIP}_{3}$ remained strongly enriched in non-compact region. Furthermore, when the level of $\mathrm{PIP}_{3}$ was increased due to the inhibition of PTEN, a dramatic morphological change was observed: an increase in the non-compact region of the oligodendrocytes. Amount of the filamentous actin was also increased in these cells at the mature stage.

We speculate, therefore, that spatial segregation of phosphatidylinositols at the early developmental stages, might have a signalling purpose: $\mathrm{PIP}_{2}$ within the sheets serves as a membrane docking point for the MBP upon its translation; whereas $\mathrm{PIP}_{3}$ enriched zones highlight the regions where MBP should not zip. We suspect filamentous actin to be a downstream-effector molecule of the $\mathrm{PIP}_{3}$ signalling, that would hinder the MBP-membrane interaction and in this way prevent compaction. 


\section{References}

Abo, S., Bernard, C. C., Webb, M., Johns, T. G., Alafaci, A., Ward, L. D., Simpson, R. J., \& Kerlero de Rosbo, N. 1993. Preparation of highly purified human myelin oligodendrocyte glycoprotein in quantities sufficient for encephalitogenicity and immunogenicity studies. Biochemistry and molecular biology international, 30(5), $945-58$.

Afar, D. E., Salzer, J. L., Roder, J., Braun, P. E., \& Bell, J. C. 1990. Differential phosphorylation of myelin-associated glycoprotein isoforms in cell culture. Journal of neurochemistry, 55(4), 1418-26.

Aggarwal, S., Yurlova, L., Snaidero, N., Reetz, C., Frey, S., Zimmermann, J., Pähler, G., Janshoff, A., Friedrichs, J., Müller, D.J., Goebel, C., \& Simons, M. 2011. A size barrier limits protein diffusion at the cell surface to generate lipid-rich myelin-membrane sheets. Developmental Cell, 21(3), 445-456.

Aggarwal, S., Snaidero, N., Pähler, G., Frey, S., Sánchez, P., Zweckstetter, M., Janshoff, A., Schneider, A., Weil, M.-T., Schaap, I. A. T., Görlich, D., \& Simons, M. 2013. Myelin membrane assembly is driven by a phase transition of myelin basic proteins into a cohesive protein meshwork. PLoS Biology, 11(6), e1001577.

Ainger, K, Avossa, D, Diana, A. S., Barry, C., Barbarese, E., \& Carson, J. H. 1997. Transport and localization elements in myelin basic protein mRNA. The Journal of cell biology, 138(5), 1077-87.

Ang, A. L., Taguchi, T., Francis, S., Fölsch, H., Murrells, L. J., Pypaert, M., Warren, G., \& Mellman, I. 2004. Recycling endosomes can serve as intermediates during transport from the Golgi to the plasma membrane of MDCK cells. The Journal of cell biology, 167(3), 531-43.

Angelis, D. A. De, \& Braun, P. E. 1996. 2',3'-cyclic nucleotide 3'-phosphodiesterase binds to actin-based cytoskeletal elements in an isoprenylation-independent manner. Journal of neurochemistry, 943-951.

Antonsson, B. 1997. Phosphatidylinositol synthase from mammalian tissues. Biochimica et biophysica acta, 1348(12), 179-86.

Arikketh, D., Nelson, R., \& Vance, J. E. 2008. Defining the importance of phosphatidylserine synthase-1 (PSS1): unexpected viability of PSS1-deficient mice. The Journal of biological chemistry, 283(19), 12888-97.

Atkins, C. M., Chen, S. J., Klann, E., \& Sweatt, J. D. 1997. Increased phosphorylation of myelin basic protein during hippocampal long-term potentiation. Journal of neurochemistry, 68(5), 1960-7.

Bakhti, M., Winter, C., \& Simons, M. 2011. Inhibition of myelin membrane sheath formation by oligodendrocytederived exosome-like vesicles. The Journal of biological chemistry, 286(1), 787-96.

Bakhti, M., Snaidero, N., Schneider, D., Aggarwal, S., Möbius, W., Janshoff, A., Eckhardt, M., Nave, Kl.-A., \& Simons, M. 2013. Loss of electrostatic cell-surface repulsion mediates myelin membrane adhesion and compaction in the central nervous system. Proceedings of the National Academy of Sciences of the United States of America, 110(8), 3143-8.

Balasubramanian, K., \& Schroit, A. J. 2003. Aminophospholipid asymmetry: a matter of life and death. Annual review of physiology, 65(Jan.), 701-34.

Balla, T., Szentpetery, Z., \& Kim, Y. J. 2009. Phosphoinositide signaling: new tools and insights. Physiology (Bethesda, Md.), 24(Aug.), 231-44.

Barbarese, E., Brumwell, C., Kwon, S., Cui, H., \& Carson, J. H. 2000. RNA on the road to myelin. Journal of neurocytology, 28(4-5), 263-70.

Barres, B. A., \& Raff, M. C. 1999. Axonal control of oligodendrocyte development. The Journal of cell biology, 147(6), 1123-8.

Baumann, N., \& Pham-Dinh, D. 2001. Biology of oligodendrocyte and myelin in the mammalian central nervous system. Physiological reviews, 81(2), 871-927.

Bennett, V., \& Healy, J. 2008. Organizing the fluid membrane bilayer: diseases linked to spectrin and ankyrin. Trends in molecular medicine, 14(1), 28-36.

Berger, T., \& Reindl, M. 2007. Multiple sclerosis: disease biomarkers as indicated by pathophysiology. Journal of the neurological sciences, 259(1-2), 21-6. 
Bieberich, E. 2012. It's a lipid's world: bioactive lipid metabolism and signaling in neural stem cell differentiation. Neurochemical research, 37(6), 1208-29.

Bifulco, M., Laezza, C., Stingo, S., \& Wolff, J. 2002. CNPase: a membrane-bound, microtubule-associated protein and membrane anchor for tubulin. PNAS, 99(4), 1807-1812.

Bilder, D., Schober, M., \& Perrimon, N. 2003. Integrated activity of PDZ protein complexes regulates epithelial polarity. Nature cell biology, 5(1), 53-8.

Boggs, J. M. 2006. Myelin basic protein: a multifunctional protein. Cellular and molecular life sciences : CMLS, 63(17), 1945-61.

Boggs, J. M., Wang, H., Gao, W., Arvanitis, D. N., Gong, Y., \& Min, W. 2004. A glycosynapse in myelin? Glycoconjugate journal, 21(3-4), 97-110.

Boggs, J. M., Gao, W., Zhao, Ji., Park, H.-J., Liu, Y., \& Basu, A. 2010. Participation of galactosylceramide and sulfatide in glycosynapses between oligodendrocyte or myelin membranes. FEBS letters, 584(9), 1771-8.

Bohdanowicz, M., \& Grinstein, S. 2013. Role of phospholipids in endocytosis, phagocytosis, and macropinocytosis. Physiological reviews, 93(1), 69-106.

Bradl, M., \& Lassmann, H. 2010. Oligodendrocytes: biology and pathology. Acta neuropathologica, 119(1), 37-53.

Braun, P. E., Angelis, D. De, Shtybel, W. W., \& Bernier, L. 1991. Isoprenoid modification permits 2', 3'-cyclic nucleotide 3'-phosphodiesterase to bind to the membranes. Journal of neuroscience research, 544, 540-544.

Braverman, N. E., \& Moser, A. B. 2012. Functions of plasmalogen lipids in health and disease. Biochimica et biophysica acta, 1822(9), 1442-52.

Brites, P., Waterham, H. R., \& Wanders, R. J. A. 2004. Functions and biosynthesis of plasmalogens in health and disease. Biochimica et biophysica acta, 1636(2-3), 219-31.

Bronstein, J. M. 2000. Function of tetraspan proteins in the myelin sheath. Current opinion in neurobiology, 10(5), $552-7$.

Brown, D. A., \& London, E. 2000. Structure and function of sphingolipid- and cholesterol-rich membrane rafts. The Journal of biological chemistry, 275(23), 17221-4.

Brunner, C., Lassmann, H., Waehneldt, T. V., Matthieu, J. M., \& Linington, C. $1989 . \quad$ Differential ultrastructural localization of myelin basic protein, myelin/oligodendroglial glycoprotein, and 2',3'-cyclic nucleotide 3'-phosphodiesterase in the CNS of adult rats. Journal of neurochemistry, 52(1), 296-304.

Bunge, R. P. 1968. Glial cells and the central myelin sheath. Physiological reviews, 48(1), $197-251$.

Burger, D., Perruisseau, G., Simon, M., \& Steck, A. J. 1992. Comparison of the N-linked oligosaccharide structures of the two major human myelin glycoproteins MAG and P0: assessment of the structures bearing the epitope for HNK-1 and human monoclonal immunoglobulin M found in demyelinating neuropathy. Journal of neurochemistry, 58(3), 854-61.

Cahoy, J. D., Emery, B., Kaushal, A., Foo, L. C., Zamanian, J. L., Christopherson, K. S., Xing, Y., Lubischer, J. L., Krieg, P. A., Krupenko, S. A, Thompson, W. J, \& Barres, B. A. 2008. A transcriptome database for astrocytes, neurons, and oligodendrocytes: a new resource for understanding brain development and function. The Journal of Neuroscience, 28(1), 264-278.

Carson, J. H., Kwon, S., \& Barbarese, E. 1998. RNA trafficking in myelinating cells. Current Opinion in Neurobiology, $\mathbf{8}(5), 607-612$.

Chalhoub, N., \& Baker, S. J. 2009. PTEN and the PI3-kinase pathway in cancer. Annual review of pathology, 4(Jan.), 127-50.

Chan, K. C., Ranwani, J., \& Moscarello, M. A. 1988. Myelin basic protein binds GTP at a single site in the N-terminus. Biochemical and biophysical research communications, 152(3), 1468-1473.

Chen, S., \& Leung, H. 2004. Chaotic spread spectrum watermarking for remote sensing images. Journal of Electronic Imaging, $\mathbf{1 3}(1), 220$.

Chen, Yi., Balasubramaniyan, V., Peng, J., Hurlock, E. C., Tallquist, M., Li, J., \& Lu, Q. R. 2007. Isolation and culture of rat and mouse oligodendrocyte precursor cells. Nature protocols, 2(5), 1044-51.

Chernoff, G. F. 1981. Shiverer: an autosomal recessive mutant mouse with myelin deficiency. The Journal of heredity, $\mathbf{7 2}(2), 128$. 
Chiavegatto, S., Sun, J., Nelson, R. J., \& Schnaar, R. L. 2000. A functional role for complex gangliosides: motor deficits in GM2/GD2 synthase knockout mice. Experimental neurology, 166(2), $227-34$.

Chou, F. C., Chou, C. H., Shapira, R., \& Kibler, R. F. 1976. Basis of microheterogeneity of myelin basic protein. The Journal of biological chemistry, 251(9), 2671-9.

Coetzee, T., Suzuki, K., \& Popko, B. 1998. New perspectives on the function of myelin galactolipids. Trends in neurosciences, 21(3), 126-30

Coffey, J. C., \& McDermott, K.W. 1997. The regional distribution of myelin oligodendrocyte glycoprotein (MOG) in the developing rat CNS: an in vivo immunohistochemical study. Journal of neurocytology, 26(3), 149-61.

Cole, N. B., Smith, C. L., Sciaky, N., Terasaki, M., Edidin, M., \& Lippincott-Schwartz, J. 1996. Diffusional mobility of Golgi proteins in membranes of living cells. Science (New York, N. Y.), 273(5276), 797-801.

Cole, R., \& Vel, J. De. 1993. Preparation of astrocyte, oligodendrocyte, and microglia cultures from primary rat cerebral cultures. Chap. Preparatio, pages 25-35 of: Fedoroff, S. (ed), Protocols for Neural Cell Culture, 3rd edn. Totowa, NJ: Humana Press.

Colello, R.J., Pott, U., \& Schwab, M. E. 1994. The role of oligodendrocytes and myelin on axon maturation in the developing rat retinofugal pathway. The Journal of neuroscience, 14(5 Pt 1), 2594-605.

Costentino, M., Pritzker, L., Boulias, C., \& Moscarello, M. A. 1994. Acylation of myelin basic protein peptide 1-21 with alkyl carboxylates 2-10 carbons long affects secondary structure and posttranslational modification. Biochemistry, 33(14), 4155-62.

Cotter, La., Ozçelik, M., Jacob, C., Pereira, J. A., Locher, V., Baumann, R., Relvas, J. B., Suter, U., Tricaud, N., \& Özçelik, M. 2010. Dlg1-PTEN interaction regulates myelin thickness to prevent damaging peripheral nerve overmyelination. Science (New York, N.Y.), 328(5984), 1415-8.

Cui, Z., Vance, J. E., Chen, M. H., Voelker, D. R., \& Vance, D. E. 1993. Cloning and expression of a novel phosphatidylethanolamine N-methyltransferase. A specific biochemical and cytological marker for a unique membrane fraction in rat liver. The Journal of biological chemistry, 268(22), 16655-63.

Czech, M. P. 2000. PIP2 and PIP3: complex roles at the cell surface. Cell, 100(6), 603-6.

Danielli, J. F., \& Davson, H. 1935. A contribution to the theory of permeability of thin films. Journal of cellular and comparative physiology, 5(4), 495-508.

de Castro, F., \& Bribián, A. 2005. The molecular orchestra of the migration of oligodendrocyte precursors during development. Brain research. Brain research reviews, 49(2), 227-41.

de Ferra, F., Engh, H., Hudson, L., Kamholz, J., Puckett, C., Molineaux, S., \& Lazzarini, R. A. 1985. Alternative splicing accounts for the four forms of myelin basic protein. Cell, $43(3 \mathrm{Pt} 2), 721-7$.

Derman, M. P., Toker, A., Hartwig, J. H., Spokes, K., Falck, J. R., Chen, C. S., Cantley, L. C., \& Cantley, L. G. 1997. The lipid products of phosphoinositide 3-kinase increase cell motility through protein kinase C. The Journal of biological chemistry, 272(10), 6465-70.

des Jardins, K. C., \& Morell, P. 1983. Phosphate groups modifying myelin basic proteins are metabolically labile; methyl groups are stable. The Journal of cell biology, 97(2), 438-46.

Devaux, P. F. 1992. Protein involvement in transmembrane lipid assymetry. Annual review of biophysics and biomolecular structure, 417-439.

Dietschy, J. M., \& Turley, S. D. 2004. Cholesterol metabolism in the central nervous system during early development and in the mature animal. Journal of lipid research, 45(8), 1375-97.

Donaldson, J. G., \& Jackson, C. L. 2000. Regulators and effectors of the ARF GTPases. Current opinion in cell biology, 12(4), 475-82.

Douglass, A. D., \& Vale, R. D. 2005. Single-molecule microscopy reveals plasma membrane microdomains created by protein-protein networks that exclude or trap signaling molecules in T cells. Cell, 121(6), 937-50.

Drummond, G. I. 1962. Hydrolysis of ribonucleoside 2',3'-cyclic phosphates by a diesterase from brain. The Journal of biological chemistry, 237(11), 3535-3539.

Dubois, C., Manuguerra, J. C., Hauttecoeur, B., \& Maze, J. 1990. Monoclonal antibody A2B5, which detects cell surface antigens, binds to ganglioside GT3 and to its 9-O-acetylated derivative. The Journal of biological chemistry, 265(5), 2797-803. 
Dupree, J. L, Girault, J.-A., \& Popko, B. 1999. Axo-glial interactions regulate the localization of axonal paranodal proteins. Journal of cell Biology, 147(6), 1145-1151.

Dyer, C. A., \& Matthieu, J. M. 1994. Antibodies to myelin/oligodendrocyte-specific protein and myelin/oligodendrocyte glycoprotein signal distinct changes in the organization of cultured oligodendroglial membrane sheets. Journal of neurochemistry, 62(2), 777-87.

Eggeling, C., Ringemann, C., Medda, R., Schwarzmann, G., Sandhoff, K., Polyakova, S., Belov, V. N., Hein, Bi., von Middendorff, C., Schönle, A., \& Hell, S. W. 2009. Direct observation of the nanoscale dynamics of membrane lipids in a living cell. Nature, 457(7233), 1159-62.

Engelman, D. M. 2005. Membranes are more mosaic than fluid. Nature, 438(7068), 578-80.

Engelman, J. A. 2009. Targeting PI3K signalling in cancer: opportunities, challenges and limitations. Nature reviews. Cancer, 9(8), 550-62.

Epand, R. M., \& Moscarello, M. A. 1982. The effects of bovine myelin basic protein on the phase transition properties of sphingomyelin. Biochimica et biophysica acta, 685(2), 230-2.

Fahy, E., Subramaniam, S., Brown, H. A., Glass, C. K., Merrill, A. H., Murphy, R. C., Raetz, C. R. H., Russell, D.W., Seyama, Y., Shaw, W., Shimizu, T., Spener, F., van Meer, G., VanNieuwenhze, M. S., White, S. H. Witztum, J. L., \& Dennis, E. A. 2005. A comprehensive classification system for lipids. Journal of lipid research, 46(5), 839-61.

Fairbanks, G., Steck, T. L., \& Wallach, D. F. 1971. Electrophoretic analysis of the major polypeptides of the human erythrocyte membrane. Biochemistry, 10(13), 2606-17.

Farooqui, A., Horrocks, L. A., \& Farooqui, T. 2000. Glycerophospholipids in brain: their metabolism, incorporation into membranes, functions, and involvement in neurological disorders. Chemistry and physics of lipids, 106(1), $1-29$.

Farrer, R G, \& Quarles, R H. 1999. GT3 and its O-acetylated derivative are the principal A2B5-reactive gangliosides in cultured O2A lineage cells and are down-regulated along with O-acetyl GD3 during differentiation to oligodendrocytes. Journal of neuroscience research, 57(3), 371-80.

Fawcett, J., \& Pawson, T. 2000. Signal transduction. N-WASP regulation-the sting in the tail. Science (New York, N.Y.), 290(5492), 725-6.

Felgner, P. L., Gadek, T. R., Holm, M., Roman, R., Chan, H. W., Wenz, M., Northrop, J. P., Ringold, G. M., \& Danielsen, M. 1987. Lipofection: a highly efficient, lipid-mediated DNA-transfection procedure. Proceedings of the National Academy of Sciences of the United States of America, 84(21), 7413-7.

Feng, W., Wu, H., Chan, Li.-N., \& Zhang, M. 2008. Par-3-mediated junctional localization of the lipid phosphatase PTEN is required for cell polarity establishment. The Journal of biological chemistry, 283(34), 23440-9.

Fernández-Murray, J. 2007. Phosphatidylcholine synthesis and its catabolism by yeast neuropathy target esterase 1 . Biochimica et Biophysica Acta, 1771, 331-336.

Fitzner, D., Schneider, A., Kippert, A., Möbius, W., Willig, K. I., Hell, S. W., Bunt, G., Gaus, K., \& Simons, M. 2006. Myelin basic protein-dependent plasma membrane reorganization in the formation of myelin. The EMBO journal, 25(21), 5037-48.

Frail, D. E., \& Braun, P. E. 1984. Two developmentally regulated messenger RNAs differing in their coding region may exist for the myelin-associated glycoprotein. The Journal of biological chemistry, 259(23), 14857-62.

Frost, E., Kiernan, B. W., Faissner, A., \& Ffrench-Constant, C. 1996. Regulation of oligodendrocyte precursor migration by extracellular matrix: evidence for substrate-specific inhibition of migration by tenascin-C. $D e-$ velopmental neuroscience, 18(4), 266-73.

Fujiwara, T., Ritchie, K., Murakoshi, H., Jacobson, K., \& Kusumi, A. 2002. Phospholipids undergo hop diffusion in compartmentalized cell membrane. The Journal of cell biology, 157(6), 1071-81.

Gambin, Y., Lopez-Esparza, R., Reffay, M., Sierecki, E., Gov, N. S., Genest, M., Hodges, R. S., \& Urbach, W. 2006. Lateral mobility of proteins in liquid membranes revisited. Proceedings of the National Academy of Sciences of the United States of America, 103(7), 2098-102.

Gao, F. B., Apperly, J., \& Raff, M. 1998. Cell-intrinsic timers and thyroid hormone regulate the probability of cell-cycle withdrawal and differentiation of oligodendrocyte precursor cells. Developmental biology, 197(1), $54-66$.

Gassama-Diagne, A., Yu, W., ter Beest, M., Martin-Belmonte, F., Kierbel, A., Engel, J., \& Mostov, K. 2006. Phosphatidylinositol-3,4,5-trisphosphate regulates the formation of the basolateral plasma membrane in epithelial cells. Nature cell biology, 8(9), 963-70. 
Gerke, V., Creutz, C. E., \& Moss, S. E. 2005. Annexins: linking Ca2+ signalling to membrane dynamics. Nature reviews. Molecular cell biology, 6(6), 449-61.

Goldstein, B., \& Macara, I. G. 2007. The PAR proteins: fundamental players in animal cell polarization. Developmental cell, 13(5), 609-22.

Gorbenko, G. P., \& Kinnunen, P. K. J. 2006. The role of lipid-protein interactions in amyloid-type protein fibril formation. Chemistry and physics of lipids, 141(1-2), 72-82.

Gorter, E., \& Grendel, F. 1925. On bimolecular layers of lipids on the chromocytes of the blood. Journal of Experimental Medicine, 41, 439-443.

Gow, A., Southwood, C. M., Li, J. S., Pariali, M., Riordan, G. P., Brodie, S. E., Danias, J., Bronstein, J. M., Kachar, B., \& Lazzarini, R. A. 1999. CNS myelin and sertoli cell tight junction strands are absent in Osp/claudin-11 null mice. Cell, 99(6), 649-59.

Gravel, M., Peterson, J., Yong, V. W., Kottis, V., Trapp, B., \& Braun, P. E. 1996. Overexpression of 2',3'-cyclic nucleotide 3'-phosphodiesterase in transgenic mice alters oligodendrocyte development and produces aberrant myelination. Molecular and cellular neurosciences, 7(6), 453-66.

Green, D. R. 2000. Apoptosis and sphingomyelin hydrolysis. The flip side. The Journal of cell biology, 150(1), F5-7.

Greer, J. M., \& Lees, M. B. 2002. Myelin proteolipid protein-the first 50 years. The international journal of biochemistry \& cell biology, 34(3), 211-5.

Gregoriadis, G. 1984. Liposome technology, volume 1: preparation of liposomes. Boca Raton, FL: CRC Press, Boca Raton, FL.

Grindstaff, Kent K, Yeaman, Charles, Anandasabapathy, Niroshana, Hsu, Shu-Chan C, Rodriguez-Boulan, Enrique, Scheller, Richard H, \& Nelson, W.James J. 1998. Sec6/8 complex is recruited to cell-cell contacts and specifies transport vesicle delivery to the basal-lateral membrane in epithelial cells. Cell, 93(5), 731-40.

Grosshans, B. L., Ortiz, D., \& Novick, P. 2006. Rabs and their effectors: achieving specificity in membrane traffic. Proceedings of the National Academy of Sciences of the United States of America, 103(32), $11821-7$.

Guardiola-Diaz, H. M., Ishii, A., \& Bansal, R. 2012. Erk1/2 MAPK and mTOR signaling sequentially regulates progression through distinct stages of oligodendrocyte differentiation. Glia, 60(3), 476-86.

Guerriero, C. J., Weixel, K. M., Bruns, J. R., \& Weisz, O. A. 2006. Phosphatidylinositol 5-kinase stimulates apical biosynthetic delivery via an Arp2/3-dependent mechanism. The Journal of biological chemistry, 281(22), $15376-84$.

Han, X., Holtzman, D. M., \& Jr, D.W. M. 2001. Plasmalogen deficiency in early Alzheimer' s disease subjects and in animal models : molecular characterization using electrospray ionization mass spectrometry. Journal of neurochemistry, 1168-1180.

Hancock, J. F., Paterson, H., \& Marshall, C. J. 1990. A polybasic domain or palmitoylation is required in addition to the CAAX motif to localize p21ras to the plasma membrane. Cell, 63(1), 133-9.

Harauz, G., Ishiyama, N., \& Bates, I. R. 2000. Analogous structural motifs in myelin basic protein and in MARCKS. Molecular and cellular biochemistry, 209(1-2), 155-63.

Harauz, G., Ishiyama, N., Hill, C., Bates, I. R., Libich, D. S., \& Farès, C. 2004. Myelin basic protein-diverse conformational states of an intrinsically unstructured protein and its roles in myelin assembly and multiple sclerosis. Micron (Oxford, England : 1993), 35(7), 503-42.

Harauz, G., Ladizhansky, V., \& Boggs, J. M. 2009. Structural polymorphism and multifunctionality of myelin basic protein. Biochemistry, 48(34), 8094-104.

Harris, T. J. C., \& Peifer, M. 2004. Adherens junction-dependent and -independent steps in the establishment of epithelial cell polarity in Drosophila. The Journal of cell biology, 167(1), 135-47.

Hartline, D. K., \& Colman, D. R. 2007. Rapid conduction and the evolution of giant axons and myelinated fibers. Current Biology: $C B, \mathbf{1 7}(1), \mathrm{R} 29-35$.

Hartwig, J. H., \& Kwiatkowski, D. J. 1991. Actin-binding proteins. Current opinion in cell biology, 3(1), 87-97.

Haugh, J. M., Codazzi, F., Teruel, M., \& Meyer, T. 2000. Spatial sensing in fibroblasts mediated by 3'- phosphoinositides. The Journal of Cell Biology, 151(6), 1269-1280.

Hawkins, P. T., Eguinoa, A., Qiu, R. G., Stokoe, D., Cooke, F. T., Walters, R., Wennström, S., Claesson-Welsh, L., Evans, T., \& Symons, M. 1995. PDGF stimulates an increase in GTP-Rac via activation of phosphoinositide 3-kinase. Current biology : CB, 5(4), 393-403. 
Hehnly, H., \& Stamnes, M. 2007. Regulating cytoskeleton-based vesicle motility. FEBS letters, 581(11), 2112-8.

Hell, S. W., \& Wichmann, J. 1994. Breaking the diffraction resolution limit by stimulated emission: stimulatedemission-depletion fluorescence microscopy. Optics letters, 19(11), 780-2.

Hell, S.W. 2009. Microscopy and its focal switch. Nature methods, 6(1), 24-32.

Henderson, R., \& Unwin, P. N. 1975. Three-dimensional model of purple membrane obtained by electron microscopy. Nature, 257(5521), 28-32.

Hodgkin, A. L., \& Huxley, A. F. 1952. Propagation of electrical signals along giant nerve fibers. Proceedings of the Royal Society of London. Series B, Containing papers of a Biological character. Royal Society (Great Britain), 140(899), 177-83.

Honda, A., Nogami, M., Yokozeki, T., Yamazaki, M., Nakamura, H., Watanabe, H., Kawamoto, K., Nakayama, K., Morris, A. J., Frohman, M. A., \& Kanaho, Y. 1999. Phosphatidylinositol 4-phosphate 5-kinase alpha is a downstream effector of the small G protein ARF6 in membrane ruffle formation. Cell, 99(5), 521-32.

Hu, Y., \& Israelachvili, J. 2008. Lateral reorganization of myelin lipid domains by myelin basic protein studied at the air-water interface. Colloids and surfaces. B, Biointerfaces, 62(1), 22-30.

Hu, Y., Doudevski, I., Wood, D., Moscarello, M., Husted, C., Genain, C., Zasadzinski, J. A., \& Israelachvili, J. 2004. Synergistic interactions of lipids and myelin basic protein. Proceedings of the National Academy of Sciences of the United States of America, 101(37), 13466-71.

Iglesias, A., Bauer, J ., Litzenburger, T., Schubart, A., \& Linington, C. 2001. T- and B-cell responses to myelin oligodendrocyte glycoprotein in experimental autoimmune encephalomyelitis and multiple sclerosis. Glia, 234(February), 220-234.

Insall, R. H., \& Weiner, O. D. 2001. PIP2, PIP3 and cell movement - similar messages, different meanings? Developmental cell, 1, 743-747.

Jana, A., \& Pahan, K. 2010. Sphingolipids in multiple sclerosis. Neuromolecular medicine, 12(4), 351-61.

Jaramillo, M. L., Afar, D. E., Almazan, G., \& Bell, J. C. 1994. Identification of tyrosine 620 as the major phosphorylation site of myelin-associated glycoprotein and its implication in interacting with signaling molecules. The Journal of biological chemistry, 269(44), 27240-5.

Jarjour, A. A., Manitt, C., Moore, S. W., Thompson, K. M., Yuh, S., \& Kennedy, T. E. 2003. Netrin-1 is a chemorepellent for oligodendrocyte precursor cells in the embryonic spinal cord. Journal of neuroscience, 23(9), 3735-3744

Johns, T. G., \& Bernard, C. C. 1999. The structure and function of myelin oligodendrocyte glycoprotein. Journal of neurochemistry, 72(1), 1-9.

Jurevics, H., Hostettler, J., Sammond, D. W., Nave, K.-A., Toews, A. D., \& Morell, P. 2003. Normal metabolism but different physical properties of myelin from mice deficient in proteolipid protein. Journal of neuroscience research, $\mathbf{7 1}(6), 826-34$.

Kachar, B., Behar, T., \& Dubois-Dalcq, M. 1986. Cell shape and motility of oligodendrocytes cultured without neurons. Cell and tissue research, 244(1), 27-38.

Kamholz, J., Toffenetti, J., \& Lazzarini, R. A. 1988. Organization and expression of the human myelin basic protein gene. Journal of neuroscience research, 21(1), 62-70.

Kaplan, M. R. 1997. Induction of sodium channel clustering by oligodendrocytes. Nature, 386(17), 724-728.

Katso, R., Okkenhaug, K., Ahmadi, K., Timms, J., \& Waterfield, M. D. 2001. Cellular function of phosphoinositide 3-kinases: implications for development, immunity, homeostasis, and cancer. Annual Review of Cell and Developmental Biology, 615-75.

Kemphues, K. J., Priess, J. R., Morton, D. G., \& Cheng, N. S. 1988. Identification of genes required for cytoplasmic localization in early C. elegans embryos. Cell, 52(3), 311-20.

Kessaris, N., Fogarty, M., Iannarelli, P., Grist, M., Wegner, M., \& Richardson, W. D. 2006. Competing waves of oligodendrocytes in the forebrain and postnatal elimination of an embryonic lineage. Nature neuroscience, $\mathbf{9}(2), 173-9$

Khuong, T. M., Habets, R. L. P., Kuenen, S., Witkowska, A., Kasprowicz, J., Swerts, J., Jahn, R., van den Bogaart, G., \& Verstreken, P. 2013. Synaptic PI(3,4,5)P3 is required for Syntaxin1A clustering and neurotransmitter release. Neuron, $\mathbf{7 7}(6), 1097-108$. 
Kinross, K. M., Montgomery, K. G., Kleinschmidt, M., Waring, P., Ivetac, I., Tikoo, A., Saad, M., Hare, L., Roh, V., Mantamadiotis, T., Sheppard, K., Ryland, G. L., Campbell, I. G., Gorringe, K. L., Christensen, J. G., Cullinane, C., Hicks, R. J., Pearson, R. B., Johnstone, R. W., McArthur, G. A., \& Phillips, W. A. 2012. An activating Pik3ca mutation coupled with Pten loss is sufficient to initiate ovarian tumorigenesis in mice. The Journal of clinical investigation, $\mathbf{1 2 2}(2), 553-7$.

Kirkpatrick, L. L., Witt, A. S., Payne, H. R., Shine, H. D., \& Brady, S. T. 2001. Changes in microtubule stability and density in myelin-deficient shiverer mouse CNS axons. The Journal of neuroscience : the official journal of the Society for Neuroscience, 21(7), 2288-97.

Klugmann, M., Schwab, M. H., Pühlhofer, A., Schneider, A., Zimmermann, F., Griffiths, I. R., \& Nave, K. A. 1997. Assembly of CNS myelin in the absence of proteolipid protein. Neuron, 18(1), 59-70.

Kolesnick, R. 1994. Signal transduction through the sphingomyelin pathway. Molecular and chemical neuropathology, 21(2-3), 287-97.

Kovacs, E. M., Ali, R. G., McCormack, A. J., \& Yap, A. S. 2002. E-cadherin homophilic ligation directly signals through Rac and phosphatidylinositol 3-kinase to regulate adhesive contacts. The Journal of biological chemistry, 277(8), 6708-18.

Kovacsovics, T. J., Bachelot, C., Toker, A., Vlahos, C. J., Duckworth, B., Cantley, L. C., \& Hartwig, J. H. 1995. Phosphoinositide 3-kinase inhibition spares actin assembly in activating platelets but reverses platelet aggregation. The Journal of biological chemistry, 270(19), 11358-66.

Kozlov, G., Lee, J., Elias, D., Gravel, M., Gutierrez, P., Ekiel, I., Braun, P. E., \& Gehring, K. 2003. Structural evidence that brain cyclic nucleotide phosphodiesterase is a member of the $2 \mathrm{H}$-phosphodiesterase superfamily. The Journal of biological chemistry, 278(46), 46021-8.

Krahn, M. P., Klopfenstein, D. R., Fischer, N., \& Wodarz, A. 2010. Membrane targeting of Bazooka/PAR-3 is mediated by direct binding to phosphoinositide lipids. Current biology : CB, 20(7), 636-42.

Kuhlmann-Krieg, S. 1988. Ultrastructural features of cultured oligodendrocytes expressing stage-specific cell-surface antigens. Brain Res., 467(2), 269-80.

Kursula, P. 2001. The current status of structural studies on proteins of the myelin sheath (Review). International journal of molecular medicine, 8(5), 475-9.

Kursula, P., Meriläinen, G., Lehto, V. P., \& Heape, A. M. 1999. The small myelin-associated glycoprotein is a zinc-binding protein. Journal of neurochemistry, 73(5), 2110-8.

Kursula, P., Lehto, V.-P., \& Heape, A. M. 2000. S100beta inhibits the phosphorylation of the L-MAG cytoplasmic domain by PKA. Molecular Brain Research, 407-410.

Kusumi, Akihiro, \& Suzuki, Kenichi. 2005. Toward understanding the dynamics of membrane-raft-based molecular interactions. Biochimica et biophysica acta, 1746(3), 234-51.

Laemli U.K. 1970. Cleavage of structural proteins during the assembly of the head of bacteriophage T4. Nature, $\mathbf{2 2 7}(15), 680-685$.

Langmuir, I. 1917. The constitution and fundamental properties of solids and liquids. II. Liquids. Journal of american chemical society, 39, 1848-1906.

Lappe-Siefke, C., Goebbels, S., Gravel, M., Nicksch, E., Lee, J., Braun, P. E., Griffiths, I. R., \& Nave, K.-A. 2003. Disruption of Cnp1 uncouples oligodendroglial functions in axonal support and myelination. Nature genetics, 33(3), 366-74.

Lassing, I., \& Lindberg, U. 1988. Evidence that the phosphatidylinositol cycle is linked to cell motility. Experimental cell research, 174(1), 1-15.

Lee, J., Gravel, M., Zhang, R., Thibault, P., \& Braun, P. E. 2005. Process outgrowth in oligodendrocytes is mediated by CNP, a novel microtubule assembly myelin protein. The Journal of cell biology, 170(4), 661-73.

Lemmon, M. A. 2008. Membrane recognition by phospholipid-binding domains. Nature reviews. Molecular cell biology, 9(2), 99-111.

Lenne, P.-F., Wawrezinieck, L., Conchonaud, F., Wurtz, O., Boned, A., Guo, X.-J., Rigneault, H., He, H.-T., \& Marguet, D. 2006. Dynamic molecular confinement in the plasma membrane by microdomains and the cytoskeleton meshwork. The EMBO journal, 25(14), 3245-56.

Li, M. O., Sarkisian, M. R., Mehal, W. Z., Rakic, P., \& Flavell, R. A. 2003. Phosphatidylserine receptor is required for clearance of apoptotic cells. Science (New York, N.Y.), 302(5650), 1560-3. 
Linardic, C. M., \& Hannun, Y. A. 1994. Identification of a distinct pool of sphingomyelin involved in the sphingomyelin cycle. The Journal of biological chemistry, 269(38), 23530-7.

Liu, J., \& Casaccia, P. 2010. Epigenetic regulation of oligodendrocyte identity. Trends in neurosciences, 33(4), $193-201$.

Machesky, L M, \& Hall, A. 1997. Role of actin polymerization and adhesion to extracellular matrix in Rac- and Rho-induced cytoskeletal reorganization. The Journal of cell biology, 138(4), 913-26.

Machesky, L. M., Mullins, R. D., Higgs, H. N., Kaiser, D. A., Blanchoin, L., May, R. C., Hall, M. E., \& Pollard, T. D. 1999. Scar, a WASp-related protein, activates nucleation of actin filaments by the Arp $2 / 3$ complex. Proceedings of the National Academy of Sciences of the United States of America, 96(7), 3739-44.

Magde, D. 1974. Fluorescence correlation spectroscopy. II. An Experimental realization. Biopolymers, 13, $29-61$.

Marguet, D., Spiliotis, E. T., Pentcheva, T., Lebowitz, M., Schneck, J., \& Edidin, M. 1999. Lateral diffusion of GFP-tagged H2Ld molecules and of GFP-TAP1 reports on the assembly and retention of these molecules in the endoplasmic reticulum. Immunity, 11(2), 231-40.

Marguet, D., Lenne, P.-F., Rigneault, H., \& He, H.-T. 2006. Dynamics in the plasma membrane: how to combine fluidity and order. The EMBO journal, 25(15), 3446-57.

Martin, O. C., \& Pagano, R. E. 1994. Internalization and sorting of a fluorescent analogue of glucosylceramide to the Golgi apparatus of human skin fibroblasts: utilization of endocytic and nonendocytic transport mechanisms. The Journal of cell biology, 125(4), 769-81.

Martin, T F. 2001. PI(4,5) $\mathrm{P}(2)$ regulation of surface membrane traffic. Current opinion in cell biology, 13(4), 493-9.

Martin-Belmonte, F., \& Mostov, K. 2007. Phosphoinositides control epithelial development. Cell cycle (Georgetown, Tex.), 6(16), 1957-61.

Mason, J. L., Langaman, C., Morell, P., Suzuki, K., \& Matsushima, G. K. 2001. Episodic demyelination and subsequent remyelination within the murine central nervous system: changes in axonal calibre. Neuropathology and applied neurobiology, 27(1), 50-8.

Massey, J. B. 2001. Interaction of ceramides with phosphatidylcholine, sphingomyelin and sphingomyelin/cholesterol bilayers. Biochimica et biophysica acta, 1510(1-2), 167-84.

Maxfield, F. R., \& Tabas, I. 2005. Role of cholesterol and lipid organization in disease. Nature, 438(7068), 612-21.

Mazumder, R., Iyer, L. M., Vasudevan, S., \& Aravind, L. 2002. Detection of novel members, structure-function analysis and evolutionary classification of the $2 \mathrm{H}$-phosphoesterase superfamily. Nucleic acids research, 30(23), $5229-43$

McLaughlin, S., Wang, J., Gambhir, A., \& Murray, D. 2002. PIP2 and proteins: interactions, organization, and information flow. Annual review of biophysics and biomolecular structure, 31(Jan.), 151-75.

Mellman, I., \& Nelson, W. J. 2008. Coordinated protein sorting, targeting and distribution in polarized cells. Nature reviews. Molecular cell biology, 9(11), 833-45.

Menegoz, M., Gaspar, P., Le Bert, M., Galvez, T., Burgaya, F., Palfrey, C., Ezan, P., Arnos, F., \& Girault, J. A. 1997. Paranodin, a glycoprotein of neuronal paranodal membranes. Neuron, 19(2), 319-31.

Mennicke, U., \& Salditt, T. 2002. Preparation of Solid-Supported Lipid Bilayers by Spin-Coating. Langmuir, 18(21), $8172-8177$.

Mikoshiba, K., Kohsaka, S., Takamatsu, K., \& Tsukada, Y. 1981. Neurochemical and morphological studies on the myelin of peripheral nervous system from Shiverer mutant mice: absence of basic proteins common to central nervous system. Brain research, 204(2), 455-60.

Min, Y., Kristiansen, K., Boggs, J. M., Husted, C., Zasadzinski, J. A., \& Israelachvili, J. 2009. Interaction forces and adhesion of supported myelin lipid bilayers modulated by myelin basic protein. Proceedings of the National Academy of Sciences of the United States of America, 106(9), 3154-9.

Morell, P., \& Radin, N. S. 1969. Synthesis of cerebroside by brain from uridine diphosphate galactose and ceramide containing hydroxy fatty acid. Biochemistry, 8(2), 506-12.

Moscarello, M. A., Wood, D. D., Ackerley, C., \& Boulias, C. 1994. Myelin in multiple sclerosis is developmentally immature. The Journal of clinical investigation, 94(1), 146-54.

Mueller, V., Ringemann, C., Honigmann, A., Schwarzmann, G., Medda, R., Leutenegger, M., Polyakova, S., Belov, V. N., Hell, S. W., \& Eggeling, C. 2011. STED nanoscopy reveals molecular details of cholesterol- and cytoskeleton-modulated lipid interactions in living cells. Biophysical Journal, 101(7), 1651-1660. 
Mueller, V., Honigmann, A., Ringemann, C., Medda, R., Schwarzmann, G., \& Eggeling, C. 2013. FCS in STED microscopy: studying the nanoscale of lipid membrane dynamics. Pages 1-38 of: Sergey Y. Tetin (ed), Methods in enzymology, vol. 519. Elsevier Inc.

Murray, N., \& Steck, A. J. 1984. Impulse conduction regulates myelin basic protein phosphorylation in rat optic nerve. Journal of neurochemistry, 43(1), 243-8.

Musse, A. A., Gao, W., Homchaudhuri, L., Boggs, J. M., \& Harauz, G. 2008. Myelin basic protein as a "PI(4,5)P2modulin": a new biological function for a major central nervous system protein. Biochemistry, 47(39), 1037210382.

Myllykoski, M., Raasakka, A., Han, H., \& Kursula, P. 2012. Myelin 2',3'-cyclic nucleotide 3'-phosphodiesterase: active-site ligand binding and molecular conformation. PloS one, $\mathbf{7}(2)$, e32336.

Nagan, N., \& Zoeller, R. A. 2001. Plasmalogens: biosynthesis and functions. Progress in lipid research, 40(3), 199-229.

Nakai, M., Obata, M., Sekiguchi, F., Kato, M., Shiro, M., Ichimura, A., Kinoshita, I., Mikuriya, Ma., Inohara, T., Kawabe, K., Sakurai, H., Orvig, C., \& Yano, S. 2004. Synthesis and insulinomimetic activities of novel mono- and tetranuclear \{oxovanadium(IV)\} complexes with 3-hydroxypyridine-2-carboxylic acid. Journal of Inorganic Biochemistry, 98(1), 105-112.

Narayanan, S. P., Flores, A. I., Wang, F., \& Macklin, W. B. 2009. Akt signals through the mammalian target of rapamycin pathway to regulate CNS myelination. The Journal of neuroscience : the official journal of the Society for Neuroscience, 29(21), 6860-70.

Nawaz, S., Kippert, A., Saab, A. S., Werner, H. B., Lang, T., Nave, K.-A., \& Simons, M. 2009. Phosphatidylinositol 4,5-bisphosphate-dependent interaction of myelin basic protein with the plasma membrane in oligodendroglial cells and its rapid perturbation by elevated calcium. The Journal of neuroscience : the official journal of the Society for Neuroscience, 29(15), 4794-807.

Nieweg, K., Schaller, H., \& Pfrieger, F. W. 2009. Marked differences in cholesterol synthesis between neurons and glial cells from postnatal rats. Journal of neurochemistry, 109(1), 125-34.

Niggli, V. 2000. A membrane-permeant ester of phosphatidylinositol-3,4, 5-trisphosphate (PIP3) is an activator of human neutrophil migration. FEBS letters, 473(2), 217-21.

Niggli, V., \& Keller, H. 1997. The phosphatidylinositol 3-kinase inhibitor wortmannin markedly reduces chemotactic peptide-induced locomotion and increases in cytoskeletal actin in human neutrophils. European journal of pharmacology, 335(1), 43-52.

Norman, S. 1961. Fatty Acids. J. Lipid Research, 2(4).

Norton, W. T., \& Poduslo, S. E. 1973. Myelination in rat brain: method of myelin isolation. Journal of neurochemistry, 21(4), 749-57.

O'Brien, J. 1965a. Fatty acid and fatty aldehyde composition of the major brain lipids in normal human gray matter, white matter, and myelin. Journal of lipid research, 6, 545-551.

O’Brien, J. S. 1965b. Stability of the Myelin Membrane. Science (New York, N.Y.), 147(3662), 1099-107.

O'Brien, J. S. O., Sampson, E. L., \& OBrien, J. S. O. 1965. Lipid composition of the normal human brain : gray matter, white matter, and myelin. Journal of lipid research, $\mathbf{6}(9), 537-544$.

O'Brien, L. E., Zegers, M. M. P., \& Mostov, K. E. 2002. Opinion: Building epithelial architecture: insights from three-dimensional culture models. Nature reviews. Molecular cell biology, 3(7), 531-7.

Omlin, F. X., Webster, H. D., Palkovits, C. G., \& Cohen, S. R. 1982. Immunocytochemical localization of basic protein in major dense line regions of central and peripheral myelin. The Journal of cell biology, 95(1), 242-8.

Owen, D. M., Williamson, D., Rentero, C., \& Gaus, K. 2009. Quantitative microscopy: protein dynamics and membrane organisation. Traffic (Copenhagen, Denmark), 10(8), 962-71.

Özçelik, M., Cotter, L., Jacob, C., Pereira, J. A., Relvas, J. B., Suter, U., \& Tricaud, N. 2010. Pals1 is a major regulator of the epithelial-like polarization and the extension of the myelin sheath in peripheral nerves. The Journal of Neuroscience, 30(11), 4120-4131.

Paltauf, F. 1994. Ether lipids in biomembranes. Chemistry and physics of lipids, 74(2), 101-39.

Papakonstanti, E. A. 2007. Methods in enzymology, osmosensing and osmosignaling. Chap. Actin cyto, page 632 of: Häussinger, D. (ed), Methods in Enzymology, vol.428 edn. Academic Press. 
Parise, L. V., Lee, J., \& Juliano, R. L. 2000. New aspects of integrin signaling in cancer. Seminars in cancer biology, $\mathbf{1 0}(6), 407-14$.

Pascher, I. 1976. Molecular arrangements in sphingolipids. Conformation and hydrogen bonding of ceramide and their implication on membrane stability and permeability. Biochimica et biophysica acta, 455(2), 433-51.

Pedraza, L., Owens, G. C., Green, L. A., \& Salzer, J. L. 1990. The myelin-associated glycoproteins: membrane disposition, evidence of a novel disulfide linkage between immunoglobulin-like domains, and posttranslational palmitylation. The Journal of cell biology, 111(6 Pt 1), 2651-61.

Pedraza, L., Huang, J. K., \& Colman, D. R. 2001. Organizing Principles of the Axoglial Apparatus. Neuron, 30, $335-344$.

Peters, A. 1964. Observations on the Connexions Between Myelin Sheaths and Glial Cells in the Optic Nerves of Young Rats. Journal of anatomy, 98(Jan.), 125-34.

Pfeiffer, S. E., Warrington, A. E., \& Bansal, R. 1993. The oligodendrocyte and its many cellular processes. Trends in cell biology, 3(6), 191-7.

Pham-Dinh, D., Della Gaspera, B., Kerlero de Rosbo, N., \& Dautigny, A. 1995 . Structure of the human myelin/oligodendrocyte glycoprotein gene and multiple alternative spliced isoforms. Genomics, 29(2), 345-52.

Poliak, S., \& Peles, E. 2003. The local differentiation of myelinated axons at nodes of Ranvier. Nature reviews. Neuroscience, 4(12), 968-80.

Prehoda, K. E., Scott, J. A., Mullins, R. D., \& Lim, W. A. 2000. Integration of multiple signals through cooperative regulation of the N-WASP-Arp2/3 complex. Science (New York, N.Y.), 290(5492), 801-6.

Privat, A., Jacque, C., Bourre, J. M., Dupouey, P., \& Baumann, N. 1979. Absence of the major dense line in myelin of the mutant mouse "shiverer". Neuroscience letters, 12(1), 107-12.

Quarles, R. H. 2002. Myelin sheaths: glycoproteins involved in their formation, maintenance and degeneration. Cellular and molecular life sciences : CMLS, 59(11), 1851-71.

Quarles, R. H. 2007. Myelin-associated glycoprotein (MAG): past, present and beyond. Journal of neurochemistry, 100(6), 1431-48.

Radin, N. S. 1984. Biosynthesis of the sphingoid bases: a provocation. Journal of lipid research, 25(13), 1536-40.

Redwine, J. M., Blinder, K. L., \& Armstrong, R. C. 1997. In situ expression of fibroblast growth factor receptors by oligodendrocyte progenitors and oligodendrocytes in adult mouse central nervous system. Journal of neuroscience research, 50(2), 229-37.

Reynolds, R., \& Wilkin, G. P. 1988. Development of macroglial cells in rat cerebellum. II. An in situ immunohistochemical study of oligodendroglial lineage from precursor to mature myelinating cell. Development (Cambridge, England), 102(2), 409-25.

Reynolds, R., \& Wilkin, G. P. 1991. Oligodendroglial progenitor cells but not oligodendroglia divide during normal development of the rat cerebellum. Journal of neurocytology, 20(3), 216-24.

Riccio, P., Fasano, A., Borenshtein, N., Bleve-Zacheo, T., \& Kirschner, D. A. 2000. Multilamellar packing of myelin modeled by lipid-bound MBP. Journal of neuroscience research, 59(4), 513-21.

Rietveld, A., \& Simons, K. 1998. The differential miscibility of lipids as the basis for the formation of functional membrane rafts. Biochimica et biophysica acta, 1376(3), 467-79.

Rios, J. C., Melendez-Vasquez, C. V., Einheber, S., Lustig, M., Grumet, M., Hemperly, J., Peles, E., \& Salzer, J. L. 2000. Contactin-associated protein (Caspr) and contactin form a complex that is targeted to the paranodal junctions during myelination. The Journal of neuroscience : the official journal of the Society for Neuroscience, 20(22), 8354-64.

Rispoli, P., Carzino, R., Svaldo-Lanero, T., Relini, A., Cavalleri, O., Fasano, A., Liuzzi, G. M., Carlone, G., Riccio, P., Gliozzi, A., \& Rolandi, R. 2007. A thermodynamic and structural study of myelin basic protein in lipid membrane models. Biophysical journal, 93(6), 1999-2010.

Rivas, A. A., Castro, R. M., \& Carmona, P. 1997. Interaction Between Myelin Basic Protein and Cholesterol as Revealed by FTIR Spectroscopy. Chap. Section VI, pages 337-338 of: Spectroscopy of Biological Molecules: Modern Trends. Springer Netherlands.

Rivas, A. A., Civera, C., Ruiz-Cabello, J., \& Castro, R. M. 1998. Interaction of Bovine Myelin Basic Protein with Cholesterol. Journal of colloid and interface science, 204(1), 9-15. 
Ro, H.-A., \& Carson, J. H. 2004. pH microdomains in oligodendrocytes. The Journal of biological chemistry, 279(35), $37115-23$.

Robertson, J. D. 1960. The molecular structure and contact relationships of cell membranes. Progress Biophysics and Biophysical Chemistry, 10, 343-418.

Rodriguez-Boulan, E., Kreitzer, G., \& Müsch, A. 2005. Organization of vesicular trafficking in epithelia. Nature reviews. Molecular cell biology, 6(3), 233-47.

Rohatgi, R., Ho, H. Y., \& Kirschner, M. W. 2000. Mechanism of N-WASP activation by CDC42 and phosphatidylinositol 4, 5-bisphosphate. The Journal of cell biology, 150(6), 1299-310.

Rosenbluth, J., Nave, K.-A., Mierzwa, A., \& Schiff, R. 2006. Subtle myelin defects in PLP-null mice. Glia, 182(May), $172-182$.

Rosetti, C. M., \& Maggio, B. 2007. Protein-induced surface structuring in myelin membrane monolayers. Biophysical journal, 93(12), 4254-67.

Rosivatz, E., Matthews, J. G., McDonald, N. Q., Mulet, X., Ho, K. K., Lossi, N., Schmid, A. C., Mirabelli, M., Pomeranz, K. M., Erneux, C., Lam, E. W-F., Vilar, R., \& Woscholski, R. 2006. A small molecule inhibitor for phosphatase and tensin homologue deleted on chromosome 10 (PTEN). ACS chemical biology, 1(12), 780-90.

Rothman, J. E. 1994. Mechanisms of intracellular protein transport. Nature, 372(6501), 55-63.

Russell, D. W., Halford, R. W., Ramirez, D. M. O., Shah, R., \& Kotti, T. 2009. Cholesterol 24-hydroxylase: an enzyme of cholesterol turnover in the brain. Annual review of biochemistry, 78(Jan.), 1017-40.

Sadava, D. E. 1993. Life: the science of biology. Boston: Boston.

Saher, G., Brügger, B., Lappe-Siefke, C., Möbius, W., Tozawa, R., Wehr, M. C., Wieland, F., Ishibashi, S., \& Nave, K.-A. 2005. High cholesterol level is essential for myelin membrane growth. Nature neuroscience, 8(4), 468-75.

Sahl, S. J., Leutenegger, M., Hilbert, M., Hell, S. W., \& Eggeling, C. 2010. Fast molecular tracking maps nanoscale dynamics of plasma membrane lipids. Proceedings of the National Academy of Sciences of the United States of America, 107(15), 6829-34.

Sakamoto, Y., Tanaka, N., Ichimiya, T., Kurihara, T., \& Nakamura, K. T. 2005. Crystal structure of the catalytic fragment of human brain 2',3'-cyclic-nucleotide 3'-phosphodiesterase. Journal of molecular biology, 346(3), $789-800$.

Salzer, J. L., \& James, L. S. 2003. Polarized Domains of Myelinated Axons. Neuron, 40(2), 297-318.

Sambrook, E. F., \& Fritsch, T. M. 2001. Molecular cloning: a laboratory manual. In: Sambrook, J. (ed), Molecular cloning: a laboratory manual. Cold Spring Harbor Laboratory Press, 3 edition.

Sánchez, I., Hassinger, L., Paskevich, P. A., Shine, H. D., \& Nixon, R. A. 1996. Oligodendroglia regulate the regional expansion of axon caliber and local accumulation of neurofilaments during development independently of myelin formation. The Journal of neuroscience : the official journal of the Society for Neuroscience, 16(16), $5095-105$.

Sasaki, T., Takasuga, S., Sasaki, J., Kofuji, S., Eguchi, S., Yamazaki, M., \& Suzuki, A. 2009. Mammalian phosphoinositide kinases and phosphatases. Progress in lipid research, 48(6), 307-43.

Sastry, P. S. 1985. Lipids of nervous tissue: composition and metabolism. Progress in lipid research, 24(2), 69-176.

Schachner, M., \& Bartsch, U. 2000. Multiple functions of the MAG (siglec-4a) in fFormation and maintenance of myelin. Glia, 165, 154-165.

Scheffzek, K., \& Welti, S. 2012. Pleckstrin homology (PH) like domains - versatile modules in protein-protein interaction platforms. FEBS letters, 586(17), 2662-73.

Schmidt, A., \& Hall, M. N. 1998. Signaling to the actin cytoskeleton. Annual review of cell and developmental biology, 14(Jan.), 305-38.

Schulte, S., \& Stoffel, W. 1993. Ceramide UDPgalactosyltransferase from myelinating rat brain: purification, cloning, and expression. Proceedings of the National Academy of Sciences of the United States of America, 90(21), 10265-9.

Sechi, A. S., \& Wehland, J. 2000. The actin cytoskeleton and plasma membrane connection: PtdIns $(4,5) \mathrm{P}(2)$ influences cytoskeletal protein activity at the plasma membrane. Journal of cell science, $113 \mathbf{P t} \mathbf{2 1}$ (Nov.), 3685-95. 
Sharma, V. P., DesMarais, V., Sumners, C., Shaw, G., \& Narang, A. 2008. Immunostaining evidence for PI(4,5)P2 localization at the leading edge of chemoattractant-stimulated HL-60 cells. Journal of leukocyte biology, 84(2), 440-7.

Sheetz, M. P., Schindler, M., \& Koppel, D. E. 1980. Lateral mobility of integral membrane proteins is increased in spherocytic erythrocytes. Nature, 285(5765), 510-1.

Shewan, A., Eastburn, D. J., \& Mostov, K. 2011. Phosphoinositides in cell architecture. Cold Spring Harbor perspectives in biology, $\mathbf{3}(8)$, a004796.

Shi, S.-H., Jan, L. Y., \& Jan, Y.-N. 2003. Hippocampal neuronal polarity specified by spatially localized $\mathrm{mPar} 3 / \mathrm{mPar6}$ and PI 3-kinase activity. Cell, 112(1), 63-75.

Shin, K., Fogg, V. C., \& Margolis, B. 2006. Tight junctions and cell polarity. Annual review of cell and developmental biology, 22(Jan.), 207-35.

Shvartsman, D. E., Kotler, M., Tall, R. D., Roth, M. G., \& Henis, Y. I. 2003. Differently anchored influenza hemagglutinin mutants display distinct interaction dynamics with mutual rafts. The Journal of cell biology, $\mathbf{1 6 3}(4), 879-88$

Siegel, J. G. 1998. Basic neurochemistry: molecular, cellular and medical aspects. 6th edn. Philadelphia: LippincottRaven; 1999.

Siegel, J. G. 2006. Myelin formation, structure and biochemistry. Chap. Myelin For of: Siegel, J. Georg (ed), Basic Neurochemistry: Molecular, Cellular and Meidical Aspects., 6th edn. Philadelphia: Lippincott-Raven: American Society for Neurochemistry.

Simons, K., \& Ikonen, E. 1997. Functional rafts in cell membranes. Nature, 387(6633), 569-72.

Simons, K., \& Vaz, W. L. C. 2004. Model systems, lipid rafts, and cell membranes. Annual review of biophysics and biomolecular structure, 33(Jan.), 269-95.

Simons, M., \& Trajkovic, K. 2006. Neuron-glia communication in the control of oligodendrocyte function and myelin biogenesis. Journal of cell science, 119(Pt 21), 4381-9.

Simons, M., Krämer, E. M., Thiele, C., Stoffel, W., \& Trotter, J. 2000. Assembly of myelin by association of proteolipid protein with cholesterol- and galactosylceramide-rich membrane domains. The Journal of cell biology, 151(1), 143-54.

Simons, M., Kramer, E.-M., Macchi, P., Rathke-Hartlieb, S., Trotter, J., Nave, K.-A., \& Schulz, J. B. 2002. Overexpression of the myelin proteolipid protein leads to accumulation of cholesterol and proteolipid protein in endosomes/lysosomes: implications for Pelizaeus-Merzbacher disease. The Journal of cell biology, 157(2), $327-36$.

Simons, M., Snaidero, N., \& Aggarwal, S. 2012. Cell polarity in myelinating glia: from membrane flow to diffusion barriers. Biochimica et biophysica acta, 1821(8), 1146-53.

Simonsen, A., Wurmser, A. E., Emr, S. D., \& Stenmark, H. 2001. The role of phosphoinositides in membrane transport. Current opinion in cell biology, 13(4), 485-92.

Sindelar, P. J., Guan, Z., Dallner, G., \& Ernster, L. 1999. The protective role of plasmalogens in iron-induced lipid peroxidation. Free radical biology \& medicine, 26(3-4), 318-24.

Singer, S. J., \& Nicolson, G. L. 1972. The fluid mosaic model of the structure of cell membranes. Science (New York, N.Y.), 175(4023), 720-31.

Smith, R. 1992. The basic protein of CNS myelin: its structure and ligand binding. Journal of neurochemistry, 59(5), 1589-608.

Solly, S. K., Thomas, J. L., Monge, M., Demerens, C., Lubetzki, C., Gardinier, M. V., Matthieu, J. M., \& Zalc, B. 1996. Myelin/oligodendrocyte glycoprotein (MOG) expression is associated with myelin deposition. Glia, 18(1), 39-48.

Sommer, I., \& Schachner, M. 1981. Monoclonal antibodies (O1 to O4) to oligodendrocyte cell surfaces: an immunocytological study in the central nervous system. Developmental biology, 83(2), 311-27.

Spady, D. K., \& Dietschy, J. M. 1983. Sterol synthesis in vivo in 18 tissues of the squirrel monkey, guinea pig, rabbit, hamster, and rat. Journal of lipid research, 24(3), 303-15.

Spassky, N., Heydon, K., Mangatal, A., Jankovski, A., Olivier, C., Queraud-lesaux, F., Goujet-zalc, C., Thomas, J. L., \& Zalc, B. 2001. Sonic hedgehog-dependent emergence of oligodendrocytes in the telencephalon : evidence for a source of oligodendrocytes in the olfactory bulb that is independent of PDGFR $\alpha$ signaling. Development, 5004, 4993-5004. 
Spiegel, I., Adamsky, K., Eshed, Y., Milo, R., Sabanay, H., Sarig-Nadir, O., Horresh, I., Scherer, S. S., Rasband, M. N., \& Peles, E. 2007. A central role for Necl4 (SynCAM4) in Schwann cell-axon interaction and myelination. Nature neuroscience, 10(7), 861-9.

Sprinkle, T. J. 1989. 2',3'-cyclic nucleotide 3'-phosphodiesterase, an oligodendrocyte-Schwann cell and myelinassociated enzyme of the nervous system. Critical reviews in neurobiology, 4(3), 235-301.

Stephens, L. R., Hughes, K. T., \& Irvine, R. F. 1991. Pathway of phosphatidylinositol(3,4,5)-trisphosphate synthesis in activated neutrophils. Nature, 351(6321), 33-9.

Stephens, L. R., Jackson, T. R., \& Hawkins, P. T. 1993. Agonist-stimulated synthesis of phosphatidylinositol(3,4,5)trisphosphate: a new intracellular signalling system? Biochimica et biophysica acta, 1179, 27-75.

Sternberger, N H, Quarles, R H, Itoyama, Y, \& Webster, H D. 1979. Myelin-associated glycoprotein demonstrated immunocytochemically in myelin and myelin-forming cells of developing rat. Proceedings of the National Academy of Sciences of the United States of America, 76(3), 1510-4.

Stingo, S., Masullo, M., Polverini, E., Laezza, C., Ruggiero, I., Arcone, R., Ruozi, E., Dal Piaz, F., Malfitano, A. M. D'Ursi, A.M., \& Bifulco, M. 2007. The N-terminal domain of 2',3'-cyclic nucleotide 3'-phosphodiesterase harbors a GTP/ATP binding site. Chemical biology \& drug design, 70(6), 502-10.

Surewicz, W. K., Epand, R. M., Epand, R. F., Hallett, F. R., \& Moscarello, M. A. 1986. Modulation of myelin basic protein-induced aggregation and fusion of liposomes by cholesterol, aliphatic aldehydes and alkanes. Biochimica et biophysica acta, 863(1), 45-52.

Suzuki, Atsushi, \& Ohno, Shigeo. 2006. The PAR-aPKC system: lessons in polarity. Journal of cell science, 119 (Pt 6), 979-87.

Tanaka, K.A. K., Suzuki, K. G. N., Shirai, Y. M., Shibutani, S. T., Miyahara, M. S. H., Tsuboi, H., Yahara, M., Yoshimura, A., Mayor, S., Fujiwara, T. K., \& Kusumi, A. 2010. Membrane molecules mobile even after chemical fixation. Nature methods, $\mathbf{7}(11), 865-6$.

Tanentzapf, G., \& Tepass, U. 2003. Interactions between the crumbs, lethal giant larvae and bazooka pathways in epithelial polarization. Nature cell biology, 5(1), 46-52.

Tanford, Charles. 1989. Ben Franklin Stilled the Waves: An Informal History of Pouring Oil on Water with Reflections on the Ups and Downs of Scientific Life in General. Duke University Press: Duke University Press.

Tanida, I., Ueno, T., \& Kominami, E. 2008. LC3 and Autophagy. Methods in molecular biology (Clifton, N.J.), 445(2), 77-88.

Temple, S., \& Raff, M. C. 1986. Clonal analysis of oligodendrocyte development in culture: evidence for a developmental clock that counts cell divisions. Cell, 44(5), 773-9.

Thiery, J. P., \& Sleeman, J. P. 2006. Complex networks orchestrate epithelial-mesenchymal transitions. Nature reviews. Molecular cell biology, $\mathbf{7}(2), 131-42$.

Tian, W., Laffafian, I., Dewitt, S., \& Hallett, M. B. 2003. Exclusion of exogenous phosphatidylinositol-3,4,5trisphosphate from neutrophil-polarizing pseudopodia: stabilization of the uropod and cell polarity. EMBO reports, $\mathbf{4}(10), 982-8$.

Timsit, S. G., Bally-Cuif, L., Colman, D. R., \& Zalc, B. 1992. DM-20 mRNA is expressed during the embryonic development of the nervous system of the mouse. Journal of neurochemistry, 58(3), 1172-5.

Tolias, K. F., Couvillon, A. D., Cantley, L. C., \& Carpenter, C. L. 1998. Characterization of a Rac1- and RhoGDIassociated lipid kinase signaling complex. Molecular and cellular biology, 18(2), 762-70.

Trotter, J., \& Schachner, M. 1989. Cells positive for the O4 surface antigen isolated by cell sorting are able to differentiate into astrocytes or oligodendrocytes. Brain research. Developmental brain research, 46(1), 11522 .

Tsai, H. H. 2003. Netrin 1 mediates spinal cord oligodendrocyte precursor dispersal. Development, 130(10), 20952105.

Tyler, W. A., Jain, M. R., Cifelli, S. E., Li, Q., Ku, L., Feng, Y., Li, H., \& Wood, T. L. 2011. Proteomic identification of novel targets regulated by the mammalian target of rapamycin pathway during oligodendrocyte differentiation. Glia, 59(11), 1754-69.

van den Bogaart, G., Meyenberg, K., Risselada, H. J., Amin, H., Willig, K. I., Hubrich, B. E., Dier, M., Hell, S. W. Grubmüller, H., Diederichsen, U., \& Jahn, R. 2011. Membrane protein sequestering by ionic protein-lipid interactions. Nature, $\mathbf{4 7 9}(7374), 552-5$. 
van Meer, G. 2005. Cellular lipidomics. The EMBO journal, 24(18), 3159-65.

van Meer, G., \& Lisman, Q. 2002. Sphingolipid transport: rafts and translocators. The Journal of biological chemistry, 277(29), 25855-8.

Vance, J. E. 2008. Phosphatidylserine and phosphatidylethanolamine in mammalian cells: two metabolically related aminophospholipids. Journal of lipid research, 49(7), 1377-87.

Vance, J. E. 2012. Dysregulation of cholesterol balance in the brain: contribution to neurodegenerative diseases. Disease models $\&$ mechanisms, 5(6), 746-55.

Vance, J. E., \& Steenbergen, R. 2005. Metabolism and functions of phosphatidylserine. Progress in lipid research, 44(4), 207-34.

Varki, A., \& Angata, T. 2006. Siglecs-the major subfamily of I-type lectins. Glycobiology, 16(1), 1R-27R.

Vartanian, T., Goodearl, A., Viehöver, A., \& Fischbach, G. 1997. Axonal neuregulin signals cells of the oligodendrocyte lineage through activation of HER4 and Schwann cells through HER2 and HER3. The Journal of cell biology, $137(1), 211-20$.

Virchow, R. 1854. Ueber das ausgebreitete Vorkommen einer dem Nervenmark analogen Substanz in den thierischen Geweben . Virchows Arch. Pathol. Anat., 6, 562-572.

Voelker, D. R. 1984. Phosphatidylserine functions as the major precursor of phosphatidylethanolamine in cultured BHK-21 cells. Biochemistry, 81(May), 2669-2673.

Voelker, D. R. 1989. Phosphatidylserine translocation to the mitochondrion is an ATP-dependent process in permeabilized animal cells. Proceedings of the National Academy of Sciences of the United States of America, 86(24), 9921-5.

von Stein, W., Ramrath, A., Grimm, A., Müller-Borg, M., \& Wodarz, A. 2005. Direct association of Bazooka/PAR3 with the lipid phosphatase PTEN reveals a link between the PAR/aPKC complex and phosphoinositide signaling. Development (Cambridge, England), 132(7), 1675-86.

Wachsmuth, M., Waldeck, W., \& Langowski, J. 2000. Anomalous diffusion of fluorescent probes inside living cell nuclei investigated by spatially-resolved fluorescence correlation spectroscopy. Journal of molecular biology, 298(4), 677-89.

Wang, J., Arbuzova, A., Hangyás-Mihályné, G., \& McLaughlin, S. 2001. The effector domain of myristoylated alaninerich $\mathrm{C}$ kinase substrate binds strongly to phosphatidylinositol 4,5-bisphosphate. The Journal of biological chemistry, 276(7), 5012-9

Wang, S., Sdrulla, A.D., Bush, G., Nofziger, D., Hicks, C., Weinmaster, G., \& Barres, B. A. 1998. Notch receptor activation inhibits oligodendrocyte differentiation. Neuron, 21, 63-75.

Wawrezinieck, L., Rigneault, H., Marguet, D., \& Lenne, P.-F. 2005. Fluorescence correlation spectroscopy diffusion laws to probe the submicron cell membrane organization. Biophysical journal, 89(6), 4029-42.

Waxman, S. G., \& Ritchie, J. M. 1993. Molecular dissection of the myelinated axon. Annals of neurology, 33(2), $121-36$

Wells, M. A., \& Dittmer, J. C. 1967. A comprehensive study of the postnatal changes in the concentration of the lipids of developing rat brain. Biochemistry, 6(10), 3169-75.

Wennström, S., Hawkins, P., Cooke, F., Hara, K., Yonezawa, K., Kasuga, M., Jackson, T., Claesson-Welsh, L., \& Stephens, L. 1994. Activation of phosphoinositide 3-kinase is required for PDGF-stimulated membrane ruffling. Current biology: $C B, \mathbf{4}(5), 385-93$.

Wilson, H. C., Onischke, C., \& Raine, C. S. 2003. Human oligodendrocyte precursor cells in vitro: phenotypic analysis and differential response to growth factors. Glia, 44(2), 153-65.

Winckler, B., Forscher, P., \& Mellman, I. 1999. A diffusion barrier maintains distribution of membrane proteins in polarized neurons. Nature, 397(6721), 698-701.

Wishart, M. J., Taylor, G. S., \& Dixon, J. E. 2001. Phoxy lipids: revealing PX domains as phosphoinositide binding modules. Cell, $\mathbf{1 0 5}(7), 817-20$.

Wodarz, A., \& Näthke, I. 2007. Cell polarity in development and cancer. Nature cell biology, 9(9), $1016-24$.

Wood, D. D., \& Moscarello, M. A. 1989. The isolation, characterization, and lipid-aggregating properties of a citrulline containing myelin basic protein. The Journal of biological chemistry, 264(9), 5121-7. 
Wood, T. L., Bercury, K. K., Cifelli, S. E., Mursch, L. E., Min, J., Dai, J., \& Macklin, W. B. 2013. mTOR: a link from the extracellular milieu to transcriptional regulation of oligodendrocyte development. ASN neuro, 5(1), $63-79$.

Wurm, C. A., Kolmakov, K., Göttfert, F., Ta, H., Bossi, M., Schill, H., Berning, S., Jakobs, S., Donnert, G., Belov, V. N., \& Hell, S.W. 2012. Novel red fluorophores with superior performance in STED microscopy. Optical Nanoscopy, 1(1), 7 .

Yamada, M., Ivanova, A., Yamaguchi, Y., Lees, M. B., \& Ikenaka, K. 1999. Proteolipid protein gene product can be secreted and exhibit biological activity during early development. The Journal of neuroscience, 19(6), $2143-51$.

Yamashita, T., Wu, Y.-P., Sandhoff, R., Werth, N., Mizukami, H., Ellis, J. M., Dupree, J. L., Geyer, R., Sandhoff, K., \& Proia, R. L. 2005. Interruption of ganglioside synthesis produces central nervous system degeneration and altered axon-glial interactions. Proceedings of the National Academy of Sciences of the United States of America, 102(8), 2725-30.

Yan, H., \& Rivkees, S. A. 2002. Hepatocyte growth factor stimulates the proliferation and migration of oligodendrocyte precursor cells. Journal of neuroscience research, 69(5), 597-606.

Yu, W., Datta, A., Leroy, P., O’Brien, L. E., Mak, G., Jou, T.-S., Matlin, K. S., Mostov, K. E., \& Zegers, M. M. P. 2005. Beta1-integrin orients epithelial polarity via Rac1 and laminin. Molecular biology of the cell, 16(2), $433-45$.

Yurlova, L., Kahya, N., Aggarwal, S., Kaiser, H.-J., Chiantia, S., Bakhti, M., Pewzner-Jung, Y., Ben-David, O., Futerman, A. H., Brügger, B., \& Simons, M. 2011. Self-segregation of myelin membrane lipids in model membranes. Biophysical Journal, 101(11), 2713-2720.

Zhang, B., Gao, Y., Moon, S. Y., Zhang, Y., \& Zheng, Y. 2001. Oligomerization of Rac1 gtpase mediated by the carboxyl-terminal polybasic domain. The Journal of biological chemistry, 276(12), 8958-67.

Zhang, L., Hellgren, L. I., \& Xu, X. 2006. Enzymatic production of ceramide from sphingomyelin. Journal of biotechnology, 123(1), 93-105.

Zhang, S., Ge, B., \& Duncan, I. D. 2000. Tracing human oligodendroglial development in vitro. Journal of neuroscience research, 429(September 1999), 421-429. 



\section{Curriculum Vitae}

Olena Steshenko

born $17^{\text {th }}$ of November 1986 in Kiev, Ukraine

current address: Münchhausenstrasse, 38, 37085, Göttingen

E-mail: steshenko@em.mpg.de

Education and Research Experience

2010-present Max-Planck Institute for Experimental Medicine, Göttingen, Germany: AG Simons. PhD thesis: Lipid organisation and dynamics in the myelin membrane sheets.

2009-2010 Genetic department University of Cambridge, Cambridge, UK: group of Cahir O'Kane. External master. Master thesis: Generation and characterisation of mutations in the Drosophila ortholog of the human neurodegenerative disease gene spatac$\sin /$ SPG11.

2008-2009 Georg-August-University, Göttingen, Germany: International $\mathrm{MSc} / \mathrm{PhD}$ Molecular Biology program. Master's examination grade $\mathrm{B}(2.00)=$ very good.

2004-2008 Taras Schevchenko National University, Kiev, Ukraine: Faculty of Biology, department of Biochemistry. Bachelor's degree in biochemistry, grade 5 (excellent); grading scale 1 to 5 (best), high honours

1994-2004 School-Gymnasia N143, Kiev, Ukraine. Secondary complete general education (Abitur), grade 5 (excellent), high honours.

Scholarships \& Awards

2012 Poster prize, second place. NeuroWIS, Rehovot, Israel, 2012. Poster title: FCS-STED to study lipid diffusion in myelin sheath.

2009-2010 Stipend International Max-Planck Research School, Göttingen, Germany.

2008 Scholarship for the young researchers, University of Konstanz, Germany.

2007 DAAD Scholarship for the young scientists, DAAD, Kiev, Ukraine. 